Florida International University FIU Digital Commons

FIU Electronic Theses and Dissertations

University Graduate School

5-19-2010

\title{
Structural Characterization of Metal Hydrides for Energy Applications
}

Lyci George

Florida International University, lgeor001@fiu.edu

DOI: $10.25148 /$ etd.FI10080413

Follow this and additional works at: https://digitalcommons.fiu.edu/etd

Part of the Other Physical Sciences and Mathematics Commons

\section{Recommended Citation}

George, Lyci, "Structural Characterization of Metal Hydrides for Energy Applications" (2010). FIU Electronic Theses and Dissertations. 233.

https://digitalcommons.fiu.edu/etd/233

This work is brought to you for free and open access by the University Graduate School at FIU Digital Commons. It has been accepted for inclusion in FIU Electronic Theses and Dissertations by an authorized administrator of FIU Digital Commons. For more information, please contact dcc@fiu.edu. 


\section{FLORIDA INTERNATIONAL UNIVERSITY}

Miami, Florida

\section{STRUCTURAL CHARACTERIZATION OF METAL HYDRIDES FOR ENERGY APPLICATIONS}

A dissertation submitted in partial fulfillment of the requirements for the degree of DOCTOR OF PHILOSOPHY

in

MATERIALS SCIENCE AND ENGINEERING

by

Lyci George 
To: Dean Amir Mirmiran

College of Engineering and Computing

This dissertation, written by Lyci George, and entitled Structural Characterization of Metal Hydrides for Energy Applications, having been approved in respect to style and intellectual content, is referred to you for judgment.

We have read this dissertation and recommend that it be approved.

Arvind Agarwal

Chunlei Wang

Gautam Sen

Surendra K. Saxena, Major Professor

Date of Defense: May 19, 2010

The dissertation of Lyci George is approved.

Dean Amir Mirmiran

College of Engineering and Computing

Interim Dean Kevin O'Shea

University Graduate School

Florida International University, 2010 


\section{ACKNOWLEDGMENTS}

First of all, I wish to thank my Major Professor Dr. Surendra Saxena for his advices, support and guidance throughout the dissertation period. I greatly acknowledge him for the opportunities availed to me during my dissertation such as to work in national synchrotron facilities and to attend many national and international conferences. Those experiences molded me towards different aspects of my goal and helped me to expand my knowledge.

I would like to thank my dissertation committee members, Dr. Arvind Agarwal, Dr. Gautam Sen and Dr. Chunlie Wang, for their suggestions and encouragement for this endeavor. I also acknowledge Dr. Jiuhua Chen for his help and useful discussuions about the hydride research. I greatly appreciate Dr. Vadym Drozd for his guidance and also his help during different stages of the experiments. I thank Dr. Andriy Durygin for making me friendly with the instruments and Dr. Selva Raju for introducing me to high pressure techniques. I always remember the efforts of Dr. Marek Tkacz, Dr. Taras Palasyuk at Polish Academy of Sciences, to teach me the high pressure gas loading technique. I appreciate Mr. Jose Herrera for his help whenever needed during the experiments. I wish to thank Dr. Maximilian Fichtner at Institute of Nanotechnology, Germany, for providing two of the samples used for the study. I extend my thanks to all other colleagues at the Center for the Study of Matter at Extreme Conditions (CeSMEC) for their help and support.

I greatly acknowledge the financial support from Florida International University graduate school in the form of doctoral evidence acquisition (DEA) fellowship and dissertation year fellowship (DYF). I would like to acknowledge the financial support 
from National Science Foundation (International Materials Institute subcontract) to carry out the PCA work and also to attend two international conferences conducted in India. I am grateful to Carnegie DOE Alliance Center (CDAC) for their financial support and also for the special allocation of beam time at Advanced Photon Source (APS).

Portions of this work were performed at HPCAT of Advanced Photon Source (APS), Argonne National Laboratory. HPCAT is supported by DOE-BES, DOE-NNSA, NSF, and the W.M. Keck Foundation. APS is supported by DOE-BES, under Contract No. DE-AC02-06CH11357. A portion of this work is based upon research conducted at the Cornell High Energy Synchrotron Source (CHESS) which is supported by the National Science Foundation and the National Institutes of Health/National Institute of General Medical Sciences under award DMR-0225180. Part of this work was also carried out at the National Synchrotron Light Source (stations X17C and X17B2), Brookhaven National Laboratory, which was supported by the U.S. Department of Energy, Office of Science, Office of Basic Energy Sciences, under Contract No. DE-AC02-98CH10886. I also thank all the beam line staffs at these synchrotron facilities especially Dr. Yue Meng, Dr. Peter Lierman, Dr. Zhiqiang Chen and Dr. Sanjit Ghose for helping with experimental setups.

I always remember all those good teachers who inspired me for this goal. I greatly acknowledge my loving husband Ved for boosting my confidence and also for his invaluable assistance at various stages of this work. I also thank my twin sister Lincy and all my friends for motivating me and being with me whenever I needed. Lastly, I would like to thank my family members for encouraging me to pursue higher studies and being supportive through out my education. 


\section{ABSTRACT OF THE DISSERTATION \\ STRUCTURAL CHARACTERIZATION OF METAL HYDRIDES \\ FOR ENERGY APPLICATIONS}

by

Lyci George

Florida International University, 2010

Miami, Florida

\section{Professor Surendra K. Saxena, Major Professor}

Hydrogen can be an unlimited source of clean energy for future because of its very high energy density compared to the conventional fuels like gasoline. An efficient and safer way of storing hydrogen is in metals and alloys as hydrides. Light metal hydrides, alanates and borohydrides have very good hydrogen storage capacity, but high operation temperatures hinder their application. Improvement of thermodynamic properties of these hydrides is important for their commercial use as a source of energy. Application of pressure on materials can have influence on their properties favoring hydrogen storage. Hydrogen desorption in many complex hydrides occurs above the transition temperature. Therefore, it is important to study the physical properties of the hydride compounds at ambient and high pressure and/or high temperature conditions, which can assist in the design of suitable storage materials with desired thermodynamic properties.

The high pressure-temperature phase diagram, thermal expansion and compressibility have only been evaluated for a limited number of hydrides so far. This situation serves as a main motivation for studying such properties of a number of 
technologically important hydrides. Focus of this dissertation was on X-ray diffraction and Raman spectroscopy studies of $\mathrm{Mg}_{2} \mathrm{FeH}_{6}, \mathrm{Ca}\left(\mathrm{BH}_{4}\right)_{2}, \mathrm{Mg}\left(\mathrm{BH}_{4}\right)_{2}, \mathrm{NaBH}_{4}, \mathrm{NaAlH}_{4}$, $\mathrm{LiAlH}_{4}, \mathrm{LiNH}_{2} \mathrm{BH}_{3}$ and mixture of $\mathrm{MgH}_{2}$ with $\mathrm{AlH}_{3}$ or $\mathrm{Si}$, at different conditions of pressure and temperature, to obtain their bulk modulus and thermal expansion coefficient. These data are potential source of information regarding inter-atomic forces and also serve as a basis for developing theoretical models. Some high pressure phases were identified for the complex hydrides in this study which may have better hydrogen storage properties than the ambient phase. The results showed that the highly compressible $\mathrm{B}-\mathrm{H}$ or $\mathrm{Al}-\mathrm{H}$ bonds and the associated bond disordering under pressure is responsible for phase transitions observed in brorohydrides or alanates. Complex hydrides exhibited very high compressibility suggesting possibility to destabilize them with pressure. With high capacity and favorable thermodynamics, complex hydrides are suitable for reversible storage. Further studies are required to overcome the kinetic barriers in complex hydrides by catalytic addition. A comparative study of the hydride properties with that of the constituting metal, and their inter relationships were carried out with many interesting features. 


\section{TABLE OF CONTENTS}

CHAPTER

PAGE

1. INTRODUCTION 1

1.1. Overview 2

1.2. Challenges 3

1.3. Purpose of the Dissertation 4

1.4. Description of Experiments and Analyses 6

2. LITTERATURE REVIEW 8

2.1. Metal Hydrides $\quad 9$

2.2. Complex Hydrides 12

2.3. Equations of State 14

2.3.1. Pressure equations of state 15

2.3.2. Thermal equations of state 16

2.4. Investigation of Hydrides under High- Pressure and Temperature 17

2.5. Applications of Hydrides 22

3. EXPERIMENTAL TECHNIQUES AND DETAILS 24

3.1. Diamond Anvil Cell Technique 24

3.1.1. Measurement of pressure in a DAC 26

3.1.2. Sample loading in the DAC 28

3.1.3. Resistive heating in a DAC 29

3.2. X-Ray Diffraction Techniques 30

3.2.1. Powder X-ray diffraction 30

3.2.2. Synchrotron X-ray source 32

3.2.3. Rotating anode X-ray generator 34

3.2.4. Indexing of X-ray diffraction patterns 36

3.3. Raman Spectroscopy 37

3.4. Analysis of Powder X-ray Diffraction Data 40

3.4.1. Rietveld method $\quad 40$

3.4.2. Le Bail method $\quad 40$

3.4.3. Data processing and analysis tools used in the study 41

4. STRUCTURAL STABILITY OF $\mathrm{Mg}_{2} \mathrm{FeH}_{6}$ UNDER HIGH PRESSURES

AND TEMPERATURES

4.1. Introduction $\quad 43$

4.2. Synthesis and Characterization of $\mathrm{Mg}_{2} \mathrm{FeH}_{6}$

4.2.1. Synthesis 45

4.2.2. Electron microscopy $\quad 45$

4.2.3. X-ray diffraction 46 
4.2.4. Computation 46

4.3. Results and Discussions $\quad 47$

4.3.1. Electron microscopy $\quad 47$

4.3.2. High pressure micro X-ray diffraction 49

4.3.3. High temperature X-ray diffraction $\quad 54$

4.3.4. Raman spectroscopy 55

5. STRUCTURAL STABILITY OF DIFFERENT POLYMORPHS OF $\mathrm{Mg}\left(\mathrm{BH}_{4}\right)_{2}$

5.1. Introduction $\quad 57$

5.2. Experimental Details $\quad 59$

5.2.1. Synthesis 59

5.2.2. High pressure micro X-ray diffraction $\quad 59$

$\begin{array}{ll}\text { 5.3. Results and Discussion } & 60\end{array}$

5.3.1. X-ray diffraction of as synthesized $\mathrm{Mg}\left(\mathrm{BH}_{4}\right)_{2} \quad 60$

$\begin{array}{ll}\text { 5.3.2. Raman spectroscopy measurements } & 64\end{array}$

5.3.2.1. As synthesized $\mathrm{Mg}\left(\mathrm{BH}_{4}\right)_{2}$

5.3.2.2. The high temperature phase of $\mathrm{Mg}\left(\mathrm{BH}_{4}\right)_{2} \quad 69$

5.3.2.3. High pressure phase of $\mathrm{Mg}\left(\mathrm{BH}_{4}\right)_{2}$

6. HIGH PRESSURE INVESTIGATION OF Ca(BH 4$)_{2}$

6.1. Introduction 73

$\begin{array}{ll}\text { 6.2. Experimental Details } & 74\end{array}$

6.2.1. Synthesis 74

6.2.2. High pressure micro X-ray diffraction $\quad 75$

6.3. Results and Discussion $\quad 75$

6.3.1. X-ray diffraction 75

6.3.2. Raman spectroscopy

7. HIGH PRESSURE INVESTIGATION AND P-T PHASE DIAGRAM OF $\mathrm{NaBH}_{4} \quad 84$

7.1. Introduction $\quad 84$

7.2. Experimental Details 86

7.3. Results and Discussion $\quad 86$

7.3.1. In situ high P-T X-ray diffraction 86

7.3.1.1. Angle dispersive micro X-ray diffraction 86

7.3.1.2. Energy dispersive micro X-ray diffraction 91

7.3.2. Raman spectroscopy 93

7.3.2.1. High pressure in situ Raman spectroscopy 93

7.3.2.2. High P-T Raman spectroscopy 98

$\begin{array}{ll}\text { 7.3.3. P-T phase diagram } & 100\end{array}$

7.3.4. Correlation of site group to factor group and Raman activity 101 
8. STRUCTURAL INVESTIGATION OF NaAlH 4 AND THE INFLUENCE OF BALL MILLING ON COMPRESSION PROPERTIES 104

8.1. Introduction 104

8.2. Unmilled $\mathrm{NaAlH}_{4} \quad 106$

8.2.1. Experimental details 106

8.2.2. Results and discussion 106

8.2.2.1. X-ray diffraction 106

$\begin{array}{ll}\text { 8.2.2.2. Raman spectroscopy } & 109\end{array}$

8.3. Mechano-chemically Milled $\mathrm{NaAlH}_{4} \quad 110$

8.3.1. Experimental details 110

$\begin{array}{ll}\text { 8.3.2. Results and discussions } & 111\end{array}$

9. HIGH PRESSURE INVESTIGATION OF STRUCTURAL STABILITY OF $\mathrm{LiAlH}_{4} \quad 112$

9.1. Introduction $\quad 115$

9.2. Experimental Details 116

9.3. Results and Discussion 116

9.3.1. X-ray diffraction 116

$\begin{array}{ll}\text { 9.3.2. Raman spectroscopy } & 118\end{array}$

10. PRINCIPAL COMPONENT ANALYSIS ON PROPERTIES OF BINARY AND TERNARY HYDRIDES $\quad 121$

$\begin{array}{ll}\text { 10.1. Introduction } & 121\end{array}$

10.2. Data 122

10.3. Method 122

10.4. Results and Discussion 125

11. INVESTIGATION OF MIXTURES OF HYDRIDES 131

11.1. High Pressure Raman Spectroscopic Investigation of $\mathrm{LiNH}_{2} \mathrm{BH}_{3}$

11.1.1. Introduction $\quad 131$

11.1.2. Results and Discussions 134

11.2. High P-T investigation of mixture of $\mathrm{MgH}_{2}$ and $\mathrm{AlH}_{3}$

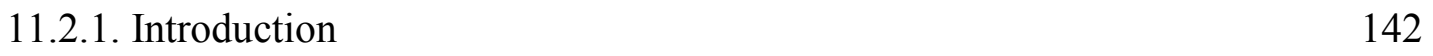

11.2.2. Results and discussions 145

11.2.2.1. X-ray diffraction 145

11.2.2.2. Raman spectroscopy 149

11.3. Mixtures of $\mathrm{Si}$ and $\mathrm{MgH}_{2} \quad 151$

$\begin{array}{ll}\text { 11.3.1. Introduction } & 151\end{array}$

11.3.2. Results and discussion 152 
12. COMPARISON OF PROPERTIES OF HYDRIDES 154

12.1. Properties of Metals and Hydrides: A Comparison 154

12.2. Inter-relationships of Thermodynamic Properties of Hydrides 160

12.3. Structural Properties of Complex Metal Hydrides 167

13. SUMMARY 172

13.1. Conclusions 172

13.2. Recommendations for Future Research 175

$\begin{array}{ll}\text { LIST OF REFERENCES } & 177\end{array}$

$\begin{array}{ll}\text { APPENDIX } & 192\end{array}$

VITA 198 


\section{LIST OF TABLES}

TABLE

PAGE

Table 2. 1: Weight, gravimetric (wt \%) and volumetric $\left(\mathrm{kg} / \mathrm{m}^{3}\right)$ hydrogen contents and decomposition temperature of alkali and alkali- earth metal hydrides.

Table 2. 2: A comparison of structure and bulk modulus values of alkali and alkali- earth metals.

Table 2. 3: Weight, gravimetric (wt \%) and volumetric $\left(\mathrm{kg} / \mathrm{m}^{3}\right)$ hydrogen content, decomposition reaction and corresponding temperature of alkali and alkali- earth complex hydrides.

Table 2. 4: A comparison of transition pressure, temperature, structure and bulk modulus data of alkali and alkali- earth metal hydrides. The data marked * is from computation results and others are experimental values.

Table 2. 5: A comparison of transition pressure, temperature, structure and bulk modulus data of alkali and alkali- earth alanates. The data marked $*$ is from computation results and others are experimental values.

Table 2. 6: A comparison of transition pressure, temperature, structure and bulk modulus of alkali and alkali- earth borohydrides. The data marked * is from computation results and others are experimental values.

Table 3. 1: High pressure apparatuses and pressure- temperature ranges that can be achieved using each of them.

Table 3. 2: Different information available from the condition of Debye rings in a 2D X-ray diffraction experiment.

Table 4. 1: Weight \% of elemental components $\mathrm{O}, \mathrm{Mg}$ and Fe obtained from EDS analysis at spots marked 1-3 in the secondary image.

Table 4. 2: Data obtained from fitting P-V data with Birch-Murnaghan EoS for various $\mathrm{Mg}_{2} \mathrm{FeH}_{6}$ samples with a comparison of pressure ranges. PTM abbreviates pressure transmitting medium.

Table 5. 1: Raman frequency shifts as a function of pressure for liberational, translational and bending modes from fitting the compression data of as synthesized sample. 
Table 5. 2: Raman frequency shifts as a function of pressure for B- $\mathrm{H}$ stretching modes during both compression and decompression of as prepared $\mathrm{Mg}\left(\mathrm{BH}_{4}\right)_{2}$.

Table 5. 3: Raman frequency shifts as a function of pressure for B-H stretching modes during both compression and decompression for the high temperature phase of $\mathrm{Mg}\left(\mathrm{BH}_{4}\right)_{2}$.

Table 6. 1: Change in Raman shift frequencies as a function of pressure for B-H stretching modes of $\mathrm{Ca}\left(\mathrm{BH}_{4}\right)_{2}$ during compression and decompression.

Table 7. 1: The observed B-H stretching modes in $\mathrm{cm}-1$ (sh- shoulder) for different phases of $\mathrm{NaBH}_{4}$.

Table 7. 2: The pressure dependence of Raman shift frequency for $\mathrm{NaBH}_{4}$ during both compression and decompression.

Table 7. 3: Symmetry analysis and irreducible representation of lattice modes of $\mathrm{NaBH}_{4}$.

Table 10. 1: The component matrix obtained from PCA of a set of data with both binary and ternary hydrides. Subscript $\mathrm{M}$ denotes metal and $\mathrm{MH}$ abbreviates metal hydride.

Table 10. 2: Correlation matrix obtained from PCA of a set of data with both binary and ternary hydrides. $\mathrm{M}$ and $\mathrm{MH}$ abbreviates metal and metal hydride, respectively.

Table 11. 1: Raman shift frequencies in cm-1 for $\mathrm{LiNH}_{2} \mathrm{BH}_{3}(\mathrm{LAB})$ in this study at ambient conditions in comparison with that of $\mathrm{NH}_{3} \mathrm{BH}_{3}(\mathrm{AB})$; str. Abbreviates stretching modes. The mode frequencies marked * are from FTIR study [229].

Table 11. 2: B-H and N-H stretching modes of the $\mathrm{LiNH}_{2} \mathrm{BH}_{3}$ in cm-1 at $4.2 \mathrm{GPa}$ as observed from Raman spectra of two different phase regions, D and W. The wave numbers given in bold are of an unidentified phase.

Table 12. 1: Approximate values of molar volumes of hydrogen atoms in boron hydrides.

Table 12. 2: Variation of the entropy of hydrogen in some binary metal hydrides (MHx).

Table 13.1. Summary of the results obtained from the high pressure and/or temperature investigations of the complex hydrides. Data marked * are of ball milled sample. 


\section{LIST OF FIGURES}

FIGURE

PAGE

Figure 1. 1: Summary of the research work performed in the dissertation.

Figure 1. 2: A comparison of the specific energy content of hydrogen with that of other fuels.

Figure 3. 1: a) A loaded Mao- Bell type DAC; b) schematic of the sample chamber in a diamond anvil cell, 1- diamond, 2- gasket hole, 3- sample, 4- gasket, 5- ruby chip.

Figure 3. 2: The shift of ruby fluorescent spectrum with the application of a pressure of $4.4 \mathrm{GPa}$.

Figure 3. 3: Drilling machine which is employed to drill holes in the gaskets using spark erosion method.

Figure 3. 4: Schematics of an X-ray diffractometer with transmission geometry; adapted from X17C hutch at NSLS [107].

Figure 3. 5: The X-ray diffractometer stage at the beam line 16 IDB- HPCAT of Argonne National Laboratory.

Figure 3. 6: A Bruker- GADDS/D8 X-ray system with molybdenum rotating anode $\mathrm{X}$-ray generator at CeSMEC.

Figure 3. 7: Raman spectroscopy setup with in situ ruby pressure measurement system at CeSMEC.

Figure 4. 1: a) Selective area electron diffraction pattern of annealed $\mathrm{Mg}_{2} \mathrm{FeH}_{6}$. b) TEM image of as synthesized sample.

Figure 4. 2: (a) Backscattered SEM image and; (b) secondary image of annealed $\mathrm{Mg}_{2} \mathrm{FeH}_{6}$.

Figure 4. 3: The EDS spectrum collected from spot 3 marked in the secondary image.

Figure 4. 4: Synchrotron XRD pattern collected at ambient conditions with the results of the Rietveld analysis. Inset is the simulated crystal structure of $\mathrm{Mg}_{2} \mathrm{FeH}_{6}$. 
Figure 4. 5: (a) Debye diffraction rings collected from sample loaded in DAC, at ambient conditions. (b) X-ray diffraction patterns of $\mathrm{Mg}_{2} \mathrm{FeH}_{6}$ for some selected pressures during compression and decompression (pressure indicated by ${ }^{*}$ ). Peaks correspond to hcp phase of Fe are marked by closed circles in the patterns at 18.9 GPa.

Figure 4. 6: A comparison of calculated and experimental variation of unit cell volume of $\mathrm{Mg}_{2} \mathrm{FeH}_{6}(\mathrm{MFH})$ with pressure fitted with the third order BirchMurnaghan EoS under different conditions. PTM abbreviates pressure transmitting medium.

Figure 4. 7: High temperature XRD patterns of the $\mathrm{Mg}_{2} \mathrm{FeH}_{6}$ collected at various temperatures. In the pattern taken at $450{ }^{\circ} \mathrm{C}$, peak marked by asterisks is due to $\mathrm{Fe}$ and that marked by closed circle is $\mathrm{MgO}$, and others are of $\mathrm{Mg}$.

Figure 4. 8: Plot of normalized volume versus temperature for $\mathrm{Mg}_{2} \mathrm{FeH}_{6}$ fitted with a second order polynomial.

Figure 4. 9: Raman spectrum of $\mathrm{Mg}_{2} \mathrm{FeH}_{6}$ at ambient conditions. Liberational modes (x); Fe-H bending modes (*); Fe-H stretching mode (o).

Figure 5. 1: Synchrotron XRD pattern of low pressure phase of $\mathrm{Mg}\left(\mathrm{BH}_{4}\right)_{2}$ at $1 \mathrm{GPa}$ with the results of Le Bail profile fitting and inset shows the corresponding hexagonal $\mathrm{P} 6_{1}$ structure.

Figure 5. 2: The synchrotron XRD pattern obtained for $\mathrm{Mg}\left(\mathrm{BH}_{4}\right)_{2}$; (a) during compression $(\lambda=0.4066 \AA)$ up to $\sim 11 \mathrm{GPa}$, (b) during decompression $(\lambda=$ $0.368138 \AA$ ) at various pressures.

Figure 5. 3: Pressure dependence of volume (circles) for the high pressure phase of $\mathrm{Mg}\left(\mathrm{BH}_{4}\right)_{2}$, during compression, fitted with third order Birch- Murnaghan EoS (line).

Figure 5. 4: The Raman spectra of $\mathrm{Mg}\left(\mathrm{BH}_{4}\right)_{2}$ collected during compression up to $21 \mathrm{GPa}$ at some selected pressures; (a) B-H bending modes, (b) B-H stretching modes. The peak around $1350 \mathrm{~cm}^{-1}$ is because of diamond in DAC.

Figure 5. 5: The Raman spectra of $\mathrm{Mg}\left(\mathrm{BH}_{4}\right)_{2}$ collected during decompression to ambient pressure; (a) B-H bending modes, (b) B-H stretching modes. Peak marked with asterisks is due to impurities in diamond. 
Figure 5. 6: Change in vibrational frequency with pressure for as prepared $\mathrm{Mg}\left(\mathrm{BH}_{4}\right)_{2}$ during compression; (a) liberational and translational modes, (b) B-H bending modes. The pressures corresponding to change in vibrational modes during compression is marked by grid lines parallel to $\mathrm{Y}$ axis.

Figure 5. 7: Change in vibrational frequency of $\mathrm{B}-\mathrm{H}$ stretching modes with pressure for as prepared $\mathrm{Mg}\left(\mathrm{BH}_{4}\right)_{2}$; (a) during compression, (b) during decompression. The pressures corresponding to change in vibrational modes during compression is marked by grid lines parallel to $\mathrm{Y}$ axis.

Figure 5. 8: (a) Raman spectra of as prepared $\mathrm{Mg}\left(\mathrm{BH}_{4}\right)_{2}$ in comparison with the high temperature phase during compression; peak marked with asterisks is due to impurities in diamond; $\mathrm{BH}$ - before heating (as synthesized); $\mathrm{AH}$ - after heating (high temperature phase). (b) Change in B-H stretching modes with pressure for the high temperature phase.

Figure 5. 9: Raman spectra of $\operatorname{Mg}\left(\mathrm{BH}_{4}\right)_{2}$; (a) as synthesized phase in comparison with the high pressure phase during compression at some selected pressures,(b) high pressure phase up to $375^{\circ} \mathrm{C}$ at some selected temperatures.

Figure 6. 1: Synchrotron X-ray diffraction pattern of the sample containing $\alpha$ and $\beta$ polymorphs of $\mathrm{Ca}\left(\mathrm{BH}_{4}\right)_{2}$ at ambient conditions; (a) Debye ring, (b) integrated plot with the results of Rietveld analysis.

Figure 6. 2: The refined crystal structures of $\mathrm{Ca}\left(\mathrm{BH}_{4}\right)_{2}$ polymorphs; $(\alpha)$ orthorhombic and ( $\beta$ ) tetragonal- $\mathrm{P} 42 / \mathrm{m}$ phases. The atoms $\mathrm{Ca}, \mathrm{B}$ and $\mathrm{H}$ are represented by symbols of color blue, red, and black, respectively.

Figure 6. 3: Synchrotron $\mathrm{XRD}$ patterns of $\mathrm{Ca}\left(\mathrm{BH}_{4}\right)_{2}$; during compression up to 13.6 GPa and after decompression to $0.5 \mathrm{GPa}$ (marked $* *$ ).

Figure 6. 4: Pressure dependence of the volume (circles) for $\alpha-\mathrm{Ca}\left(\mathrm{BH}_{4}\right)_{2}$ during compression, fitted with the third-order Birch-Murnaghan equation of state (line).

Figure 6. 5: Raman spectrum (blue line) of $\mathrm{Ca}\left(\mathrm{BH}_{4}\right)_{2}$ containing both $\alpha$ (yellow colored peaks) and $\beta$ (black colored peaks) polymorphs collected at ambient conditions.

Figure 6. 6: Raman spectra of $\mathrm{Ca}\left(\mathrm{BH}_{4}\right)_{2}$; (a) during compression up to $25 \mathrm{GPa}$, (b) during decompression for B-H stretching modes. The peak (marked *) around 2650 $\mathrm{cm}^{-1}$ is because of diamond in the DAC. 
Figure 6. 7: Change in the Raman shift with pressure for B-H stretching modes of $\mathrm{Ca}\left(\mathrm{BH}_{4}\right)_{2}$; (a) during compression and (b) during decompression. The pressures corresponding to the change in vibrational modes are marked by grid lines parallel to the $\mathrm{Y}$ axis.

Figure 7. 1: Micro X-ray diffraction pattern of the cubic phase of $\mathrm{NaBH}_{4}$ collected at $0.8 \mathrm{GPa}$ using a synchrotron source with the results of Rietveld analysis. The peak positions marked in green (a) corresponds to that of $\mathrm{NaBH}_{4}$ and that marked in pink (b) are of $\mathrm{NaCl}$.

Figure 7. 2: Selected XRD patterns of the three different phases of the $\mathrm{NaBH}_{4}$, at room temperature; (a) the cubic $(3.4 \mathrm{GPa})$, tetragonal $(6.5 \mathrm{GPa})$ and orthorhombic $(9 \mathrm{GPa}) .(\mathrm{b})$ at various temperatures and $6.5 \mathrm{GPa}$. Between 323 and $373 \mathrm{~K}$, tetragonal to cubic phase transition occurs. Peaks marked * are of $\mathrm{NaCl}$ which was used as a pressure marker.

Figure 7. 3: Crystal structures of (a) the cubic, (b) tetragonal and (c) orthorhombic phases of $\mathrm{NaBH}_{4}$. The $\mathrm{Na}, \mathrm{B}$, and $\mathrm{H}$ are marked in green, orange and black symbols, respectively.

Figure 7. 4: Selected high pressure XRD patterns of $\mathrm{NaBH}_{4}$ above $13.4 \mathrm{GPa}$ up to $34 \mathrm{GPa}$. The arrow indicated new peaks, and decompressed pattern is marked *.

Figure 7. 5: An EDXRD pattern of cubic- $\mathrm{NaBH}_{4}$ at $0.2 \mathrm{GPa}$. The peaks marked $(*),(\mathrm{o})$ and (\#) are due to $\mathrm{BN}, \mathrm{NaCl}$ and $\mathrm{K} \alpha$, respectively.

Figure 7. 6: Pressure dependence of the unit cell volume (circles) for the highpressure phase of cubic $\mathrm{NaBH}_{4}$ during compression at room temperature and at 573 $\mathrm{K}$, fitted with third order Birch- Murnaghan EoS (line).

Figure 7. 7: Raman spectra of $\mathrm{NaBH}_{4}$ during compression; (a) at selected pressures and the spectrum marked ? corresponds to the appearance of a new phase, (b) spectra in the range 14-30 GPa.

Figure 7. 8: Selected Raman spectra of $\mathrm{NaBH}_{4}$ during decompression.

Figure 7. 9: The Raman shift versus pressure obtained by peak fitting of B-H stretching modes at each pressure; (a) during compression and (b) decompression. Pressures corresponds to phase transition are marked with grid lines parallel to the $\mathrm{Y}$ axis. 
Figure 7. 10: The high temperature Raman spectra of $\mathrm{NaBH}_{4}$ in the $\mathrm{B}-\mathrm{H}$ stretching mode region; (a) while heating at $9.5 \mathrm{GPa}$ in comparison with a spectrum at $3 \mathrm{GPa}$, (b) at $14 \mathrm{GPa}$ and different temperatures.

Figure 7. 11: P-T phase diagram of $\mathrm{NaBH}_{4}$ investigated through DAC technique using XRD and Raman spectroscopic measurements (circles) including the results of low temperature study of Sundqvist et al. [169], (asterisks). The phase regions marked $\mathrm{C}, \mathrm{T}, \mathrm{O}$ and $\mathrm{M}$ are cubic, tetragonal, orthorhombic and monoclinic, respectively. The region marked ? implies structure of phase is not confirmed.

Figure 8. 1: The XRD patterns of unmilled $\mathrm{NaAlH}_{4}$ recorded at various pressures up to $13.6 \mathrm{GPa}$. Peaks due to orthorhombic phase are marked by asterisks at 12.4 GPa.

Figure 8. 2: The P-V plot of unmilled $\mathrm{NaAlH}_{4}$ (circles) fitted with BirchMurnaghan equation of state (line).

Figure 8. 3: Raman spectra of unmilled $\mathrm{NaAlH}_{4}$ at various pressures up to $15 \mathrm{GPa}$. The change in Raman modes under pressure are marked with arrow.

Figure 8. 4: The XRD patterns of the ball-milled $\mathrm{NaAlH}_{4}$ collected at various pressures. Pattern marked * is from decompressed sample, and Pt metal was used as a pressure marker.

Figure 8. 5: Birch Murnaghan EoS fit (line) to the P-V data (circles) of ball milled $\mathrm{NaAlH}_{4}$.

Figure 9. 1: Comparison of the high pressure XRD patterns of $\mathrm{LiAlH}_{4}$ collected at room temperature.

Figure 9. 2: The P-V data (circles) obtained for monoclinic phase of $\mathrm{LiAlH}_{4}$ fitted with third order Birch-Murnaghan EoS (line).

Figure 9. 3: Raman spectrum of $\mathrm{LiAlH}_{4}$ in at ambient conditions (dotted line) fitted with a Gaussian profile function.

Figure 9. 4: Raman spectra of $\mathrm{LiAlH}_{4}$ collected at various pressures and at room temperature.

Figure 10. 1: Eigen value and total variance explained by each component obtained from PCA of a set of data with both binary and ternary metal hydrides. 
Figure 10. 2: Component plots for a set of data with both binary and ternary hydrides. Subscript $\mathrm{M}$ denotes metal and $\mathrm{MH}$ denotes metal hydride. S- entropy, $\mathrm{Cp}$ - specific heat, $\mathrm{V}$ - molar volume and $\mathrm{H}$ wt \%- hydrogen weight percent in each compound.

Figure 10. 3: Plot of score 2 versus score 1 of a set of data with both binary and ternary hydrides.

Figure 10. 4: Plot of scores of first two principal components for binary hydrides.

Figure 10. 5: Plot of scores of first two principal components for ternary hydrides.

Figure 11. 1: Lattice, bending and B-H stretching modes in the Raman spectrum of $\mathrm{LiNH}_{2} \mathrm{BH}_{3}$, collected at ambient conditions fitted with a Gaussian profile function.

Figure 11. 2: N-H stretching modes in the Raman spectrum of $\mathrm{LiNH}_{2} \mathrm{BH}_{3}$, collected at ambient conditions fitted with a Gaussian profile function.

Figure 11. 3: The sample loaded in DAC with two phase regions marked $\mathrm{W}$ and $\mathrm{D}$ at different pressures. $\mathrm{DP}_{0}$ implies decompressed sample at ambient conditions.

Figure 11. 4: Raman spectra of $\mathrm{LiNH}_{2} \mathrm{BH}_{3}$ during compression (right) and decompression (left) for the regions W (top) and D (bottom), respectively.

Figure 11. 5: Change of Raman shift with pressure for $\mathrm{LiNH}_{2} \mathrm{BH}_{3}$ during compression of B-H (left) and N-H (right) stretching modes for the regions $\mathrm{W}$ (top) and D (bottom), respectively.

Figure 11. 6: The micro XRD patterns of mixture of $\mathrm{AlH}_{3}$ and $\mathrm{MgH}_{2}$ collected at various pressures and room temperature. Abbreviations: bh- before heating, ahafter heating.

Figure 11. 7: The XRD pattern from decompressed sample at room temperature after heating under pressure up to $9 \mathrm{GPa}$ matched with peaks corresponds to $\mathrm{MgH}_{2}$ (shorter lines) and $\mathrm{Al}$ (longer lines).

Figure 11. 8: XRD patterns of $\mathrm{Mg}\left(\mathrm{AlH}_{4}\right)_{2}$ containing $\mathrm{LiCl}($ marked *) at some selected pressures.

Figure 11. 9: Raman spectra of the $2 \mathrm{AlH}_{3}+\mathrm{MgH}_{2}$ mixture collected at various pressures in comparison with the Raman spectra of $\mathrm{Mg}\left(\mathrm{AlH}_{4}\right)_{2}$. 
Figure 11. 10: Raman spectra of $\operatorname{Mg}\left(\mathrm{AlH}_{4}\right)_{2}$ collected at various pressures. Spectrum marked * is of the decompressed sample and 'ah' abbreviates after heating.

Figure 11. 11: (a) The XRD pattern of the mixture of $\mathrm{Si}$ and $\mathrm{MgH}_{2}$ in comparison to that of pure components. (b) Raman spectra of $\mathrm{MgH}_{2}+$ Si mixture before and after heating at $0.5 \mathrm{GPa}$ and $600{ }^{\circ} \mathrm{C}$ and that of pure $\mathrm{Mg}_{2} \mathrm{Si}$; bh and ah abbreviates before and after heating, respectively.

Figure 12. 1: Comparison of molar volume of binary hydrides and corresponding metal.

Figure 12. 2: Molar volume of Li containing hydrides versus the element sum [70, $71,73,83,170,247-249]$.

Figure 12. 3: A comparison of entropy versus molar volume of metals and metal hydrides. Subscript M denotes metal and MH denotes metal hydride.

Figure 12. 4: Plots of entropy of binary metal hydrides versus that of corresponding metals.

Figure 12. 5: Plot of specific heat of various binary metal hydrides versus that of the host metal. The dotted line corresponds to the specific heat of metals.

Figure 12. 6: A comparison of molar volumes $\left(\mathrm{cm}^{3} / \mathrm{mol}\right)$ of various alkali hydrides with that of the corresponding host metals.

Figure 12. 7: Variation of entropy with molar volume of various hydrides [62, 249253].

Figure 12. 8: The variation of hydrogen content versus molar volume for the rareearth metal hydrides.

Figure 12. 9: The variation of specific heat versus molar volume for binary metal hydrides $[70,71,73,83,170]$.

Figure 12. 10: Plot of enthalpy versus molar volume of alkali and alkali- earth metal hydrides.

Figure 12. 11: Plot of bulk modulus versus molar volume of alkali and alkali- earth metal hydrides. 
Figure 12. 12: Plot of decomposition temperature versus density of alkali and alkali- earth metal hydrides.

Figure 12. 13: Plot of decomposition temperature versus density of alkali and alkali- earth complex hydrides.

Figure 12. 14: Plot of bulk modulus versus molar volume of alkali and alkali- earth complex hydrides.

Figure 12. 15: A comparison of the change of molar volume with pressure for the complex hydrides investigated in this study. 


\section{CHAPTER 1. INTRODUCTION}

This dissertation focuses on investigation of the structural stability of light hydrides with high gravimetric and volumetric hydrogen storage capacities under high pressures or temperatures. The goal of this study, as summarized in Figure 1.1, is to obtain thermodynamic and physical properties of hydrides which are important to determine the factors affecting their stability.

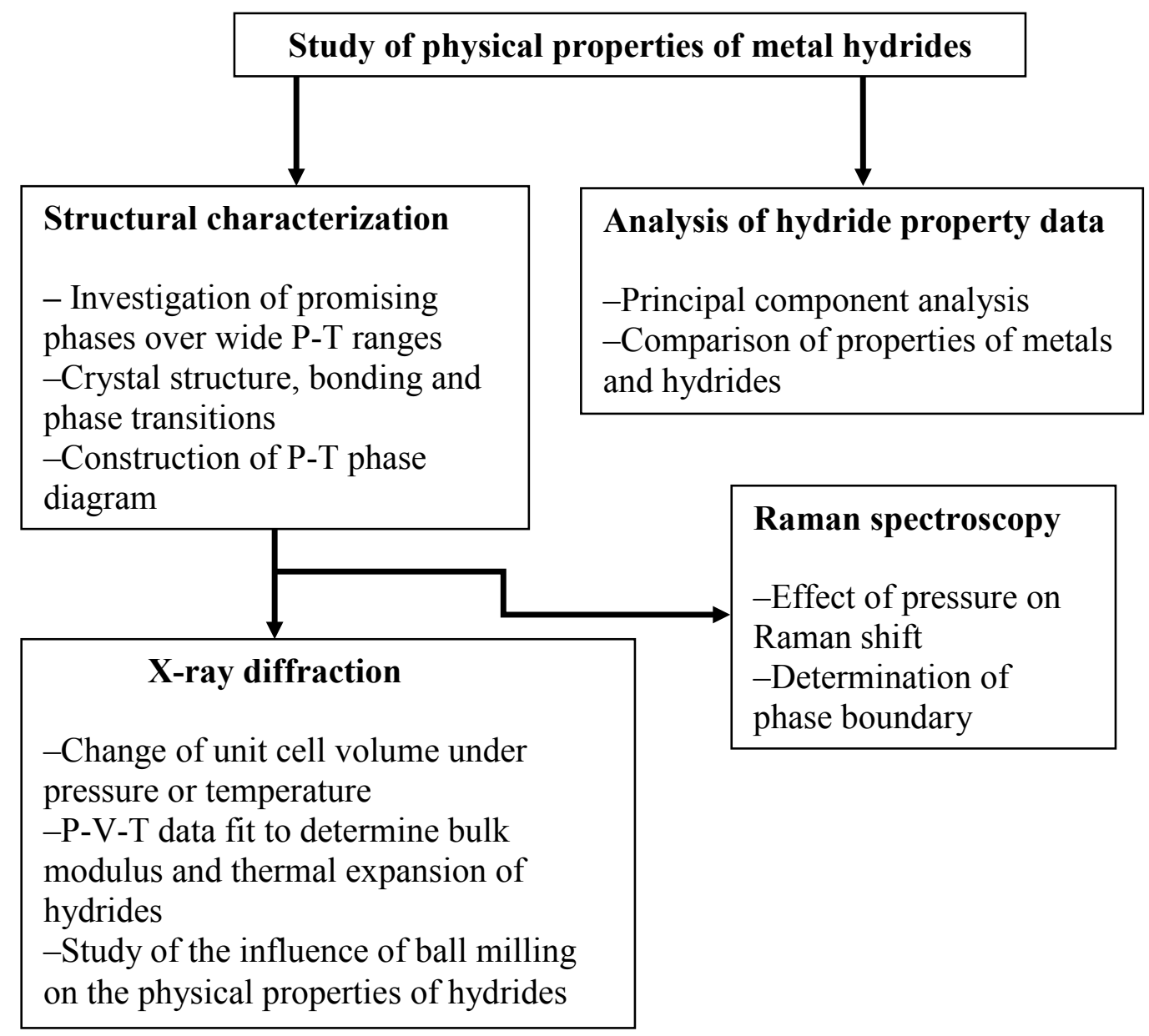

Figure 1. 1: Summary of the research work performed in the dissertation. 


\subsection{Overview}

The depletion of fossil fuels, increase in pollution and related environmental hazards require us to discover alternate energy sources. Hydrogen is a zero emission fuel with very high specific energy content of about $120 \mathrm{MJ} / \mathrm{kg}$ and an energy density of $10 \mathrm{Wh} / \mathrm{kg}$. A comparison of energy contents of various fuels are illustrated in Figure 1.2. Storage is a challenging issue that cuts across production, delivery and commercial applications of hydrogen as an energy carrier. Use of pure $\mathrm{H}_{2}$ is not economical as it requires high pressure gas cylinders that can handle pressures up to $80 \mathrm{MPa}$, or cryogenic tanks which have to be maintained at $21 \mathrm{~K}$. Hydrogen stored in solid materials are safe alternative to liquid or compressed form and convenient for long time storage with high yield. Hydrogen storage in materials can be categorized into two types;

- Metal and complex hydrides (eg. $\mathrm{LiH}, \mathrm{LiNH}_{2}, \mathrm{NaBH}_{4}, \mathrm{Mg}\left(\mathrm{AlH}_{4}\right)_{2}$ etc.), - High surface area sorbents (eg. carbon based materials, clatherates, conducting polymers, nanotubes/fibers, metal-organic frameworks and aero gels).

Among the above discussed hydrogen storage materials, hydrides are interesting because of their high volumetric and gravimetric density. The fuel cell technology requires hydrides of high hydrogen storage densities with moderate hydrogenation kinetics. 


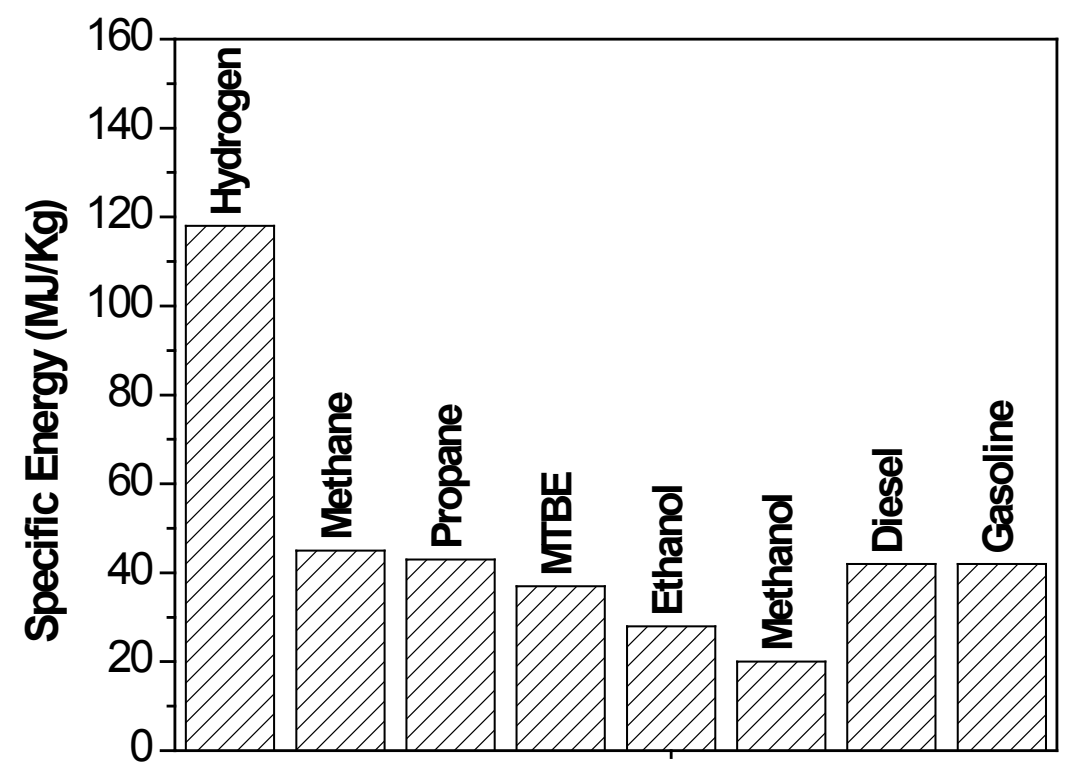

Figure 1. 2: A comparison of the specific energy content of hydrogen with that of other fuels.

\subsection{Challenges}

A hydrogen storage material should release $\mathrm{H}_{2}$ at low temperature with wide range of operation, high volumetric density and mass density. A hydride should also have rapid hydrogenation/dehydrogenation rates, high resistance to oxidation, high capacity, low cost, good reversibility, fast reactivity and sustainability. Main issues of hydrides are weight, volume, cost, safety, efficiency, refueling time and durability. Minimum requirements for automobile applications of hydrides are:

○ Gravimetric energy density: $2 \mathrm{kWh} / \mathrm{kg}$

○ Volumetric energy density: $1.5 \mathrm{kWh} /$ liter

$\circ \mathrm{H}_{2}$ storage capacity: $6 \mathrm{wt} \%$,

○ Operating temperature: 30 to $50{ }^{\circ} \mathrm{C}$ 
○ Refueling time: $<5 \mathrm{~min}$

○ Refueling rate: $1.5 \mathrm{~kg} \mathrm{H}_{2} / \mathrm{min}$

- Recoverable amount of hydrogen: $90 \%$

○ Cycle life: 500 times

○ Cost target: US $\$ 5 / \mathrm{kWh}$ (without peripheral components)

The transport sector requires not only cheap, safe and reliable way of storing hydrogen but also high storage capacity, fast kinetics and favorable thermodynamics. A potential hydrogen storage candidate has yet to be discovered, which meets all the mentioned criteria. One of the high-priority goals of the Department of Energy is "finding effective hydrogen storage materials" which they list as one of the most difficult challenges of commercial use of hydrides for transportation. Light metal hydrides have good hydrogen storage capacity but operation temperature is very high while transition metal hydrides release $\mathrm{H}_{2}$ at room temperature but with less storage capacity. Therefore it is important to study thermodynamic stability of light hydrides in order to understand possibilities to improve the temperature of operation. Study of physical properties of hydrides under pressure/temperature conditions can assist in the design of suitable storage materials.

\subsection{Purpose of the Dissertation}

The high pressure-temperature phase diagram, thermal expansion and compressibility have only been evaluated for a limited number of hydrides so far. This situation serves as a main motivation for studying such properties of a number of technologically important hydrides. The application of pressure on materials can have influence on their properties 
favoring hydrogen storage. Study of hydrides under pressure is interesting because it can help to understand atomic, electronic and structural properties of hydrogen which in turn will shed light on improving the storage properties. The high pressure investigations can reveal if a polymorph with higher storage capacity can be retained at ambient conditions.

This dissertation is targeting high volumetric and gravimetric storage capacities as the criteria for selection of hydride materials for the study. Eventhough the selected hydrides have high hydrogen storage capacity, their dehydrogenation properties are not good due to kinetic barriers. To modify the hydrides for commercial applications we need to understand the crystal structure, bonding and related properties. Crystal structure, compressibility and thermal expansion of a number of complex hydrides (eg., $\mathrm{LiAlH}_{4}$, $\mathrm{NaAlH}_{4}, \mathrm{NaBH}_{4}, \mathrm{Mg}_{2} \mathrm{FeH}_{6}, \mathrm{Mg}\left(\mathrm{BH}_{4}\right)_{2}$, and $\left.\mathrm{Ca}\left(\mathrm{BH}_{4}\right)_{2}\right)$ has been studied at high pressures using diamond anvil cell (DAC) technique and observed interesting new phases for most of them. Reactions of $\mathrm{MgH}_{2}$ with $\mathrm{AlH}_{3}$ and Si under P-T conditions, and structural phase trasitions of $\mathrm{LiNH}_{2} \mathrm{BH}_{3}$ also have been investigated. The main focus is on $\mathrm{Mg}$ and $\mathrm{Al}$ based hydrides as they are light and abundant materials, and has relatively low operating temperatures. Alanates and borohydrides of alkali or alkali-earth metals have improved thermodynamic and kinetic properties as compared to their metal hydride counterparts. Structural measurements on the hydride compounds at ambient and high pressure or temperature conditions can result in a better understanding of the stability of the hydride structures and can assist us in the design of suitable storage materials with desired thermodynamic properties. Furthermore, it is reported that the crystallite size and processing conditions influence the $\mathrm{H}_{2}$ sorption kinetics of the hydrogen storage materials. The investigation of the influence of crystallite size is essential in order to 
obtain a reliable reference for evaluation of the kinetic effects of doping with catalytic additives. Therefore, the effect of ball milling on the properties of $\mathrm{Mg}_{2} \mathrm{Fe}_{2} \mathrm{H}_{6}$ and $\mathrm{NaAlH}_{4}$ also has been investigated. Hydrides are light materials with low scattering power and the use of diamond anvil cell limits the amount of sample we can use. In order to resolve dynamical structural changes under pressure, high quality data is required and hence the micro X-ray diffraction using synchrotron radiation source is availed for the study. To confirm the phase transitions and to obtain the accurate transition pressures, Raman spectroscopic study also has been carried out.

\subsection{Description of Experiments and Analyses}

The main focus of this dissertation is to investigate the structural phase transitions of selected hydrides at high pressures and to determine their thermodynamic properties.

- Micro X-ray diffraction studies of structural phase transitions under high pressures up to 10-35 GPa for $\mathrm{LiAlH}_{4}, \mathrm{NaAlH}_{4}, \mathrm{NaBH}_{4}, \mathrm{Mg}_{2} \mathrm{FeH}_{6}, \mathrm{Mg}\left(\mathrm{BH}_{4}\right)_{2}$ and $\mathrm{Ca}\left(\mathrm{BH}_{4}\right)_{2}$, using synchrotron radiation source.

- Raman spectroscopic investigation of structural stability under high pressures up to 30 GPa for $\mathrm{LiAlH}_{4}, \mathrm{NaAlH}_{4}, \mathrm{NaBH}_{4}, \mathrm{Mg}\left(\mathrm{BH}_{4}\right)_{2}, \mathrm{Ca}\left(\mathrm{BH}_{4}\right)_{2}$ and $\mathrm{LiNH}_{2} \mathrm{BH}_{3}$.

- Thermal decomposition studies of $\mathrm{Mg}_{2} \mathrm{FeH}_{6}$ in the range $25-500{ }^{\circ} \mathrm{C}$ in quartz capillary and high pressure phase of $\mathrm{Mg}\left(\mathrm{BH}_{4}\right)_{2}$ in a DAC using resistive heating.

- Construction of P-T phase diagram for $\mathrm{NaBH}_{4}$ using resistive heating $(\mathrm{P}<15 \mathrm{GPa}, \mathrm{T}<$ $500{ }^{\circ} \mathrm{C}$ ) in the DAC from the results of micro X-ray diffraction and Raman spectroscopic studies. 
- Investigation of the effect of ball milling as well as that of mineral oil and silicone oil on the physical properties of $\mathrm{NaAlH}_{4}$ and $\mathrm{Mg}_{2} \mathrm{FeH}_{6}$.

- Micro structural characterization of $\mathrm{Mg}_{2} \mathrm{FeH}_{6}$ using scanning and transmission electron microscopy techniques.

- Determination of the change of lattice parameters and hence the molar volume with pressure and temperature using Rietveld analysis or Le Bail fitting methods.

- Determination of bulk modulus and thermal expansion coefficient using equation of state fit to the unit cell volume data as a function of pressure and temperature, respectively.

- Systematic investigation of frequency shift with pressure to determine the boundary of phase transition in the Raman spectroscopy results.

○ A comparison of the properties of hydrides to find any interrelationship among them.

- The investigation of correlation among the properties of hydrides with the help of principal component analysis.

$\circ$ Investigation of mixtures $2 \mathrm{AlH}_{3}+\mathrm{MgH}_{2}$ and $3 \mathrm{MgH}_{2}+\mathrm{Si}$ under pressure and temperature as a route to synthesize complex hydrides.

The discussion of this work is divided into different chapters in the dissertation which include introduction, literature review, experimental procedures, results and discussion of each hydrides selected for the study, conclusions and recommendations for future research. The first pages of the publications arising out of this research work are included in the appendix. 


\section{CHAPTER 2. LITTERATURE REVIEW}

Metal can absorb $\mathrm{H}_{2}$ until it attains equilibrium and the reaction rate is a function of pressure and temperature. In most cases, a structural transformation occurs when the material absorbs $\mathrm{H}_{2}$ due to isotropic cell volume expansion. Hydrogen desorption from hydrides may proceed through thermal decomposition of the hydride at the hydride-metal interface, interstitial diffusion of hydrogen through metal phase, surface penetration of hydrogen atoms, recombination of chemisorbed hydrogen atoms and physisorption, and molecular hydrogen degassing [1]. Hydrogen sorption/desorption rate and corresponding activation energies depend on applied pressure, temperature, surface contamination, crystallite size and additives or catalysts. In most cases, the rate-limiting factor cannot be determined in a straightforward manner. It is believed that hydrogen desorption is not controlled by diffusion or any phase transformation, but by a process involving hydrogen transport through the surface. Minimum inter nuclear distance between $\mathrm{H}$ - atoms should be $2.1 \AA$ which limits the number of interstitial atoms. Therefore, in crystals only octrahedral and tetrahedral sites can be occupied by H-atoms.

A stronger $\mathrm{H}-\mathrm{M}$ bond than the $\mathrm{H}-\mathrm{H}$ bond implies chemisorption is exothermic and a stronger $\mathrm{H}-\mathrm{H}$ bond as compared to $\mathrm{H}-\mathrm{M}$ bond means chemisorption is endothermic. A solid solution is formed by penetration of $\mathrm{H}$-atoms into the subsurface metal atomic layer through an activated process from which it can diffuse into the bulk of the metal. Kleperis et al. [2], have stated that the enthalpy $(\Delta \mathrm{H})$ of hydride formation should be between - 25 $\mathrm{kJ} \mathrm{mol}^{-1}$ and $-50 \mathrm{~kJ} \mathrm{~mol}^{-1}$, for an alloy to be suitable for battery applications. When $\Delta \mathrm{H}<$ $-15 \mathrm{~kJ} \mathrm{~mol}^{-1}$, the alloy hydride is not stable enough for charging the metal hydride 
electrode at room temperature. For practical applications, hydrides should release hydrogen in the temperature range $60-120{ }^{\circ} \mathrm{C}$ and pressure below $10 \mathrm{MPa}$. The operation temperature and pressure are related by the van't Hoff equation; $\ln p=\frac{\Delta H}{R T}+\frac{\Delta S}{\mathrm{R}}$, where $\mathrm{H}$ and $\mathrm{S}$ are absorption/ desorption enthalpy and entropy, respectively. Heat of formation and decomposition of the hydrides are important to estimate the operation temperature and pressure. Hydrides are mainly classified into metal hydrides and complex hydrides depending on their bonding nature.

\subsection{Metal Hydrides}

The light metal hydrides are good candidates for use as hydrogen storage materials. Alkali and alkali- earth metal hydrides have good hydrogen storage capacity ( $>12.6 \mathrm{wt}$ \%) but their operation temperature is very high, while transition metal hydrides release $\mathrm{H}_{2}$ at room temperature but with less storage capacity $(<2.5 \mathrm{wt} \%)$. Therefore, it is important to study thermodynamic stability of metal hydrides in order to understand possibilities to improve their temperature of operation. The hydrogen storage capacity and decomposition temperature for various alkali and alkali-earth metal hydrides are listed in Table 2.1. Metal hydrides can be synthesized directly from corresponding metals under high hydrogen pressure because of increased hydrogen solubility [3-8]. Bonding in metal hydrides can be ionic (eg. alkali metal hydrides), covalent (eg. $\mathrm{AlH}_{3}, \mathrm{CuH}$ etc.) or metallic (eg. transition metal and rare earth metal hydrides). The alkali metal hydrides have high degree of ionic nature (i.e. the electrons are localized around the atomic nuclei) and thus high decomposition temperatures. 
Table 2. 1: Weight, gravimetric (wt \%) and volumetric $\left(\mathrm{kg} / \mathrm{m}^{3}\right)$ hydrogen contents and decomposition temperature of alkali and alkali- earth metal- hydrides.

\begin{tabular}{lllll}
\hline Hydride & $\begin{array}{l}\text { Weight } \\
(\mathbf{g} / \mathbf{m o l})\end{array}$ & $\begin{array}{l}\mathbf{H} \\
(\mathbf{w t} \%)\end{array}$ & $\begin{array}{l}\mathbf{H} \\
\left(\mathbf{k g} / \mathbf{m}^{\mathbf{3}}\right)\end{array}$ & $\begin{array}{l}\mathbf{T}_{\mathbf{d}} \mathbf{[ 9 ]} \\
\left({ }^{\mathbf{0}} \mathbf{C}\right)\end{array}$ \\
\hline $\mathrm{LiH}$ & 7.95 & 12.59 & 98.60 & 720 \\
$\mathrm{NaH}$ & 23.99 & 4.17 & 57.73 & 425 \\
$\mathrm{KH}$ & 40.11 & 2.49 & 36.01 & 417 \\
$\mathrm{RbH}$ & 86.47 & 1.18 & 30.44 & 170 \\
$\mathrm{CsH}$ & 133.91 & 0.75 & 25.86 & 170 \\
$\mathrm{BeH}_{2}$ & 11.01 & 18.16 & 138.08 & 250 \\
$\mathrm{MgH}_{2}$ & 26.31 & 7.60 & 110.03 & 327 \\
$\mathrm{CaH}_{2}$ & 42.09 & 4.75 & 92.37 & 600 \\
$\mathrm{SrH}_{2}$ & 89.62 & 2.23 & 74.00 & 675 \\
$\mathrm{BaH}_{2}$ & 139.34 & 1.44 & 60.41 & 675 \\
\hline
\end{tabular}

The alkaline earth metal hydrides include $\mathrm{BeH}_{2}$ with covalent bonding character (due to a small difference in electro-negativity between beryllium and hydrogen) and ionic hydrides of $\mathrm{CaH}_{2}, \mathrm{SrH}_{2}$, and $\mathrm{BaH}_{2}$ [9-11]. But $\mathrm{MgH}_{2}$ is having a more complex bonding character. Generally the electronic density is located around the atoms with a net charge of $\mathrm{Mg}$ close to +2 and $\mathrm{H}$ atoms with a charge of -0.94 to $-0.26[12,13]$. The remaining electron density is distributed evenly in the interstitial region. The recent experiments have revealed regions with increased electron density between $\mathrm{Mg}$ and $\mathrm{H}$ atoms clearly suggesting a partial covalent bonding character to $\mathrm{MgH}_{2}$ [12]. At ambient conditions except $\mathrm{Be}$ and $\mathrm{Mg}$, all other alkali and alkali-earth group metals have cubic structure (Table 2.2). Both Be and Mg crystallize in the hexagonal close packed (hcp) structure but transforms into an orthorhombic structure and a tetragonal structure, respectively, for the corresponding hydrides. Due to lower atomic packing density compared to the close packed hosts the density of the hydrides of $\mathrm{Be}$ and $\mathrm{Mg}$ are lower 
than the metals. $\mathrm{Ca}, \mathrm{Ba}$, and $\mathrm{Sr}$ all have cubic crystal systems and their hydrides have orthorhombic structures $[13,14]$.

Table 2. 2: A comparison of structure and bulk modulus values of alkali and alkaliearth metals.

\begin{tabular}{|c|c|c|c|}
\hline Metal & Structure/ space group & $\begin{array}{l}\mathbf{K}_{\mathbf{0}}(\mathrm{GPa}) \\
{[15]}\end{array}$ & $\mathbf{K}_{\mathbf{0}}^{\prime}$ \\
\hline $\mathrm{Li}$ & Cubic/ $\operatorname{Im}^{-3} 3 \mathrm{~m}$ & 11.6 & 3.51 \\
\hline $\mathrm{Na}$ & Cubic/ $\mathrm{Im}^{-3} 3 \mathrm{~m}$ & 6.3 & 4.13 \\
\hline $\mathrm{K}$ & Cubic/ $\mathrm{Fm}^{-3} \mathrm{~m}$ & 3.1 & 3.91 \\
\hline $\mathrm{Rb}$ & Cubic/ $\operatorname{Im}^{-3} 3 \mathrm{~m}$ & 2.5 & 3.62 \\
\hline Cs & Cubic/ $\mathrm{Fm}^{-3} \mathrm{~m}$ & 1.57 & 3.79 \\
\hline $\mathrm{Be}$ & Hexagonal/ $\mathrm{P} 6_{3} / \mathrm{mmc}$ & 100.3 & - \\
\hline $\mathrm{Mg}$ & Hexagonal/ $\mathrm{P}_{3} / \mathrm{mmc}$ & 45 & 4.8 \\
\hline $\mathrm{Ca}$ & Cubic/ $\mathrm{Fm}^{-3} 3 \mathrm{~m}$ & 15.2 & 2.5 \\
\hline $\mathrm{Sr}$ & Cubic/ $\mathrm{Fm}^{-3} \mathrm{~m}$ & 11.61 & 2.5 \\
\hline $\mathrm{Ba}$ & Cubic/ $\mathrm{Im}^{-3} 3 \mathrm{~m}$ & 9.6 & 2.1 \\
\hline
\end{tabular}

The hydrides of the alkali- earth metals are insulators with band gaps in the range 3-6 eV for $\mathrm{Be}, \mathrm{Mg}$, and $\mathrm{Ca}[11,13,14]$. Alkali metal hydrides have cubic structure similar to the metals, and except $\mathrm{MgH}_{2}$ (tetragonal) all other alkali-earth metal hydrides exist in orthorhombic structure. A comparative study of properties of metal and corresponding hydrides suggests decrease or increase of entropy, molar volume and specific heat of hydrides from that of the metals can be attributed to hydrogen bond formation, charge transfer and corresponding change in crystal structure [16]. Metal hydrides are not considered as practical hydrogen storage material as such, due to their stability arising from ionic bonding. Both additives and processing like ball milling are found to be improving the kinetics of the hydrides [17-21]. 


\subsection{Complex Hydrides}

Binary metal hydrides of the alkali metal and alkali earth metal hydrides do not fulfill the hydrogen storage requirement of operation temperature below $120^{\circ} \mathrm{C}$. To achieve potentially improved thermodynamics it is necessary to form complex hydrides with addition of Al, B etc., to alkali or alkali earth metal hydrides. Complex hydrides are salts in which molecular anions contain hydride ions. Complex metal hydrides recently got much notice as a promising material due to their high hydrogen storage capacity and lower production costs. The hydrogen storage capacities, possible decomposition reaction and corresponding temperatures are listed in Table 2.3. Alanates are less stable than corresponding borohydrides and the decomposition temperature increases as size of metal increases from Li to Cs for alkali borohydrides. Also, B-H bond energy is larger than the Al-H bond energy [22]. Due to strong covalent and ionic bonding nature, dissociation temperatures of borohydrides are very high. The improvement of the hydride properties by catalytic addition requires better understanding of the phases and their structural stability.

Nature of chemical bonds in the complex hydrides of the form $\mathrm{MXH}_{\mathrm{n}}$, eg. $\mathrm{NaAlH}_{4}$, $\mathrm{LiBH}_{4}, \mathrm{LiNH}_{2}$ etc., was computationally studied by Yoshino et al. [22]. Their results show covalent bonding between $\mathrm{X}$ and $\mathrm{H}$ ions in the $\left[\mathrm{XH}_{\mathrm{n}}\right]$ - complex which in turn has an ionic bonding with the metal ion $\mathrm{M}^{+}$. Computational studies of $\mathrm{Mg}\left(\mathrm{AlH}_{4}\right)_{2}$ show the bonding within $\left[\mathrm{AlH}_{4}\right]^{-}$complex involves hybridization between $\mathrm{Al}$ and $\mathrm{H}$ orbital but with a strong ionic character [23]. The destabilization of alkali alanates by Ti is reported to be caused by the rearrangements of the tetrahedral $\left[\mathrm{AlH}_{4}\right]^{-}$ion into octahedral $\left[\mathrm{AlH}_{6}\right]^{3-}$ 
ion [24]. Complex hydrides are good solid electrolytes because of their high ionic conductivity [25].

Table 2. 3: Weight, gravimetric (wt \%) and volumetric $\left(\mathrm{kg} / \mathrm{m}^{3}\right)$ hydrogen content, decomposition reaction and corresponding temperature of alkali and alkali- earth complex hydrides.

\begin{tabular}{|c|c|c|c|c|c|c|}
\hline Hydride & $\begin{array}{l}\text { Wt }(g / l \\
\text { mol) }\end{array}$ & $\begin{array}{l}\text { H } \\
\text { Wt \% }\end{array}$ & $\begin{array}{l}\mathbf{H}(\mathrm{kg} / \\
\left.\mathrm{m}^{3}\right)\end{array}$ & Decomposition reaction & $\begin{array}{l}T_{d} \\
\left({ }^{\circ} \mathrm{C}\right)\end{array}$ & ref. \\
\hline $\mathrm{LiAlH}_{4}$ & 37.95 & 10.55 & 74.02 & $\begin{array}{l}3 \mathrm{LiAlH}_{4} \rightarrow \mathrm{Li}_{3} \mathrm{AlH}_{6}+2 \mathrm{Al}+3 \mathrm{H}_{2} \\
\mathrm{Li}_{3} \mathrm{AlH}_{6} \rightarrow 3 \mathrm{LiH}+\mathrm{Al}+3 / 2 \mathrm{H}_{2} \\
3 \mathrm{LiH} \rightarrow 3 \mathrm{Li}+3 / 2 \mathrm{H}_{2}\end{array}$ & $\begin{array}{l}112 \\
127 \\
720\end{array}$ & {$[26]$} \\
\hline $\mathrm{NaAlH}_{4}$ & 53.97 & 7.41 & 97.41 & $\begin{array}{l}3 \mathrm{NaAlH}_{4} \rightarrow \mathrm{Na}_{3} \mathrm{AlH}_{6}+2 \mathrm{Al}+3 \mathrm{H}_{2} \\
\mathrm{Na}_{3} \mathrm{AlH}_{6} \rightarrow 3 \mathrm{NaH}+\mathrm{Al}+3 / 2 \mathrm{H}_{2} \\
3 \mathrm{NaH} \rightarrow 3 \mathrm{Na}+3 / 2 \mathrm{H}_{2}\end{array}$ & $\begin{array}{l}220 \\
250 \\
425\end{array}$ & $\begin{array}{l}{[27-} \\
29]\end{array}$ \\
\hline $\mathrm{KAlH}_{4}$ & 70.08 & 5.71 & 70.31 & $\begin{array}{l}3 \mathrm{KAlH}_{4} \rightarrow \mathrm{K}_{3} \mathrm{AlH}_{6}+2 \mathrm{Al}+3 \mathrm{H}_{2} \\
\mathrm{~K}_{3} \mathrm{AlH} \mathrm{H}_{6} \rightarrow 3 \mathrm{KH}+\mathrm{Al}+3 / 2 \mathrm{H}_{2} \\
3 \mathrm{KH} \rightarrow 3 \mathrm{~K}+3 / 2 \mathrm{H}_{2}\end{array}$ & $\begin{array}{l}300 \\
340 \\
417\end{array}$ & {$[30]$} \\
\hline $\mathrm{RbAlH}_{4}$ & 116.45 & 3.44 & 124.4 & & & \\
\hline $\mathrm{CsAlH}_{4}$ & 163.88 & 2.44 & 108.9 & & & \\
\hline $\mathrm{Be}\left(\mathrm{AlH}_{4}\right)_{2}$ & 71.04 & 11.35 & -- & & & \\
\hline $\operatorname{Mg}\left(\mathrm{AlH}_{4}\right)_{2}$ & 86.3 & 9.27 & 98.15 & $\begin{array}{l}\text { a. } \mathrm{Mg}\left(\mathrm{AlH}_{4}\right)_{2} \rightarrow \mathrm{MgH}_{2}+2 \mathrm{Al}+3 \mathrm{H}_{2} \\
\mathrm{MgH} \mathrm{H}_{2} \rightarrow \mathrm{Mg}+\mathrm{H}_{2} \\
2 \mathrm{Al}+\mathrm{Mg} \rightarrow 1 / 2 \mathrm{Al}_{3} \mathrm{Mg}_{2}+1 / 2 \mathrm{Al} \\
\text { b. } \mathrm{Mg}(\mathrm{AlH})_{2} \rightarrow \mathrm{MgAlH}_{5}+\mathrm{Al}+1.5 \mathrm{H}_{2} \\
\mathrm{MgAlH}_{5}+\mathrm{Al} \rightarrow \mathrm{MgH}_{2}+2 \mathrm{Al}+1.5 \mathrm{H}_{2}\end{array}$ & $\begin{array}{l}110-200 \\
240-380 \\
400 \\
120-155 \\
210-255\end{array}$ & $\begin{array}{l}{[31,} \\
32]\end{array}$ \\
\hline $\mathrm{Ca}\left(\mathrm{AlH}_{4}\right)_{2}$ & 102.04 & 7.84 & 96.97 & $\begin{array}{l}\mathrm{Ca}\left(\mathrm{AlH}_{4}\right)_{2} \rightarrow \mathrm{CaAlH}_{5}+\mathrm{Al}+3 / 2 \mathrm{H}_{2} \\
\mathrm{CaAlH}_{5} \rightarrow \mathrm{CaH}_{2}+\mathrm{Al}+3 / 2 \mathrm{H}_{2} \\
\mathrm{CaH}_{2} \rightarrow \mathrm{Ca}+\mathrm{H}_{2}\end{array}$ & $\begin{array}{l}200 \\
260-550 \\
600-700\end{array}$ & {$[33]$} \\
\hline $\operatorname{Sr}\left(\mathrm{AlH}_{4}\right)_{2}$ & 149.65 & 5.39 & -- & & & \\
\hline $\mathrm{Ba}\left(\mathrm{AlH}_{4}\right)_{2}$ & 199.36 & 4.044 & -- & & & \\
\hline $\mathrm{LiBH}_{4}$ & 21.75 & 18.39 & 124 & $\mathrm{LiBH}_{4} \rightarrow \mathrm{LiH}+\mathrm{B}+3 / 2 \mathrm{H}_{2}$ & 400 & {$[34]$} \\
\hline $\mathrm{NaBH}_{4}$ & 37.81 & 10.58 & 118 & $\mathrm{NaBH}_{4} \rightarrow \mathrm{Na}+\mathrm{B}+2 \mathrm{H}_{2}$ & 565 & {$[35]$} \\
\hline $\mathrm{KBH}_{4}$ & 53.90 & 7.42 & 85.57 & & 500 & [9] \\
\hline $\mathrm{RbBH}_{4}$ & 100.28 & 3.99 & 77.2 & & 600 & [9] \\
\hline $\mathrm{CsBH}_{4}$ & 147.72 & 2.71 & 65.66 & & 660 & [9] \\
\hline $\mathrm{Be}\left(\mathrm{BH}_{4}\right)_{2}$ & 38.69 & 20.84 & 126.9 & $\mathrm{Be}\left(\mathrm{BH}_{4}\right)_{2} \rightarrow \mathrm{Be}+2 \mathrm{~B}+4 \mathrm{H}_{2}$ & & {$[36]$} \\
\hline $\mathrm{Mg}\left(\mathrm{BH}_{4}\right)_{2}$ & 53.93 & 14.84 & 117.2 & $\begin{array}{l}\mathrm{Mg}\left(\mathrm{BH}_{4}\right)_{2} \rightarrow \mathrm{MgH}_{2}+2 \mathrm{~B}+3 \mathrm{H}_{2} \\
\mathrm{MgH}_{2} \rightarrow \mathrm{Mg}+\mathrm{H}_{2}\end{array}$ & $\begin{array}{l}290 \\
317\end{array}$ & $\begin{array}{l}{[37,} \\
38]\end{array}$ \\
\hline $\mathrm{Ca}\left(\mathrm{BH}_{4}\right)_{2}$ & 69.70 & 11.48 & 124.0 & $\begin{array}{l}\mathrm{Ca}\left(\mathrm{BH}_{4}\right)_{2} \rightarrow \mathrm{CaH}_{2}+2 \mathrm{~B}+3 \mathrm{H}_{2} \\
\mathrm{CaH}_{2} \rightarrow \mathrm{Ca}+\mathrm{H}_{2}\end{array}$ & $347-497$ & [39] \\
\hline $\mathrm{Sr}\left(\mathrm{BH}_{4}\right)_{2}$ & 117.24 & 6.88 & -- & & & \\
\hline $\mathrm{Ba}\left(\mathrm{BH}_{4}\right)_{2}$ & 166.95 & 4.79 & -- & & 375 & [9] \\
\hline
\end{tabular}




\subsection{Equations of State}

The phase change in solids at high pressure usually involves a change in crystal structure or in the atomic arrangements. Co-ordination number $(\mathrm{z})$ is important in the high pressure studies of phase transition. This is because; thermodynamically the molar volume of high pressure phase must be smaller than that of the low pressure phase. The reduction in molar volume is achieved either by a more efficient packing of co-ordination polyhedra or by an increase in co-ordination number or by both. Theoretically all materials become metallic at extremely high pressures. A variation in pressure $(\mathrm{P})$, temperature $(\mathrm{T})$ or composition $(\mathrm{x})$ can cause a phase transition in materials. In solidsolid phase transformations, generally $\mathrm{T}$ and $\mathrm{P}$ remain constant while entropy $(\mathrm{S})$ and molar volume (V) undergo finite changes [40]. Phase transitions involve change in entropy and volume $\left(\Delta \mathrm{S}_{\mathrm{t}}\right.$ and $\left.\Delta \mathrm{V}_{\mathrm{t}}\right)$ according to the Clapeyron equation (Eq. 1).

$$
\frac{d T}{d P}=\frac{\Delta V_{t}}{\Delta S_{t}}
$$

This equation implies that phase transition is induced either by a change in $\mathrm{T}$ at constant $\mathrm{P}$ or by a change in $\mathrm{P}$ at a constant $\mathrm{T}$. In the first order phase transitions the value of $\Delta \mathrm{S}_{\mathrm{t}}$ for temperature induced transition must be positive, where as $\Delta \mathrm{V}_{\mathrm{t}}$ may be any value. While for pressure induced transitions as $\mathrm{P}$ increases $\Delta \mathrm{V}_{\mathrm{t}}$ must be negative but there will not be any restriction on the values of $\Delta S_{t}$. In the case of second order phase transitions, phase changes occur at constant $\mathrm{P}$ and $\mathrm{T}$ with no volume or entropy changes. Both first and second order phase transitions must exhibit finite changes in the heat capacity $\left(C_{p}\right)$, compressibility $(\beta)$ and thermal expansion $(\alpha)$ which is expressed by the Ehrenfest's equation (Eq. 2) 
$\frac{d T}{d P}=\frac{\left(\alpha_{f}-\alpha_{i}\right) V T}{C_{p_{f}}-C_{p_{i}}}=\frac{\beta_{f}-\beta_{i}}{\alpha_{f}-\alpha_{i}}$

Past few years, researchers have attempted to reveal the properties of materials in terms of universal relationship with minimum parameters. A fair knowledge of the behavior of thermodynamic parameters at elevated pressure is significant for devising a system independent equation of state $(\mathrm{EoS})$ of materials. A mathematical expression defining the physical state of homogeneous material which relates thermodynamic parameters $\mathrm{P}, \mathrm{V}$ and $\mathrm{T}$ is referred to as an EoS.

\subsubsection{Pressure equations of state}

When the applied pressure is high, the number of nearest neighbor atoms increases as the core repulsions dominates which results in efficiently packed structures. At equilibrium volume the Madelung energy gives the dominant contribution to the total energy [41]. The change in volume during the phase transition can be computed if the EoS for the state is known. EoS can provide analytical expressions for cohesive energy which is an important thermodynamic parameter. EoS is widely applied in engineering and scientific research to get information on non-linear compression of materials at high

pressure. During the last few decades, much work has been carried out to determine an EoS which uses volume as a function of pressure and thus obtain the various energies directly as a function of pressure and temperature for thermodynamic calculations. It was a long awaited goal for researchers to find an EoS with the ability to predict compression curve of a material only using its compression properties at low pressures. Isothermal EoS comprises $\mathrm{K}_{0}$ (isothermal bulk modulus), $\mathrm{K}_{0}{ }^{\prime}$ and $\mathrm{K}_{0}{ }^{\prime \prime}$ (first and second derivatives of 
$\mathrm{K}_{0}$ with pressure, respectively). The most commonly used EoSs for fitting isothermal P-V datasets are due to Murnaghan and Birch. The Murnaghan EoS [42] can be derived from the assumption that the bulk modulus varies linearly with pressure $\mathrm{K}=\mathrm{K}_{0}+\mathrm{P} \cdot \mathrm{K}_{0}$, where $\mathrm{K}_{0}{ }^{\prime}$ being independent of pressure, integration of which yields the P-V relationship as given in Eq.3.

$$
P=\left(\frac{K_{0}}{K_{0}^{\prime}}\right)\left[\left(\frac{V_{0}}{V}\right)^{K_{\theta}^{\prime}}-1\right]
$$

This EoS reproduces P-V data and yields correct values of the room pressure bulk modulus for compressions up to about $10 \%$ (i.e. $\mathrm{V} / \mathrm{V}_{0}>0.9$ ), and has the advantage of algebraic simplicity over other formulations such as the Vinet or Birch- Murnaghan EoS [43], which should be used if the range of compression is greater than $10 \%$. BirchMurnaghan EoS [44] is fairly good up to compressions of $0.85\left(\mathrm{~V} / \mathrm{V}_{0}\right)$. This is a finite strain EoS, and is based upon the assumption that the strain energy of a solid undergoing compression can be expressed as a Taylor series which on expansion to fourth-order in the strain yields the Eq. 4 .

$$
P=\left(\frac{3}{2} K_{0}\right)\left[\left(\frac{V_{0}}{V}\right)^{\frac{7}{3}}-\left(\frac{V_{0}}{V}\right)^{\frac{5}{3}}\right]\left\{1-\frac{3}{4}\left(4-K_{0}^{\prime}\right)\left[\left(\frac{V_{0}}{V}\right)^{\frac{2}{3}}-1\right]\right\}
$$

\subsubsection{Thermal equations of state}

The high-temperature value of the zero-pressure volume can be obtained by integrating the expression of thermal expansion coefficient $(\alpha)$ which gives Eq. 5 . 


$$
V_{0}(T)=V_{0}\left(T_{0}\right) \exp \int \alpha(T) d T
$$

At the lowest level of approximation $\alpha(T)$ can be considered a constant, or varying linearly with temperature as $\alpha(T)=\alpha_{0}+\alpha_{1} T$. From the temperature dependent $\alpha$ zero pressure volume obtained can be expressed as Eq.6.

$$
\mathrm{V}_{0}(\mathrm{~T})=\mathrm{V}_{0}\left(\mathrm{~T}_{0}\right) \exp \left[\alpha_{0}\left(\mathrm{~T}-\mathrm{T}_{0}\right)+(1 / 2) \alpha_{1}\left(\mathrm{~T}^{2}-\mathrm{T}_{0}^{2}\right)\right]
$$

In this case, the actual values of $\alpha_{0}$ and $\alpha_{1}$ that describe a V-T curve depend on the value of the reference temperature, $T_{0}$. As per the experimental results the variation of bulk modulus with temperature is considered to be linear (Eq. 7) and can be used to fit most experimental P-V-T data sets up to around $725^{\circ} \mathrm{C}$.

$$
\mathrm{K}(\mathrm{T})=\mathrm{K}_{0}+\left(\mathrm{T}-\mathrm{T}_{0}\right)(\mathrm{dK} / \mathrm{dT})_{\mathrm{p}}
$$

\subsection{Investigation of Hydrides under High- Pressure and Temperature}

The structural investigations at different temperatures and pressures can help to identify various polymorphs of the material which may have better thermodynamics for hydrogen storage. X-ray diffraction, neutron diffraction and Raman spectroscopy are powerful tools to detect phase transition at elevated pressures and temperatures. Some complex hydrides like $\mathrm{LiBH}_{4}$ release $\mathrm{H}_{2}$ only above the transition temperature. In such cases it is important to understand the structural stability of high temperature phase to calculate its hydrogen desorption energy [45]. Diamond anvil cell technique is generally used to apply high pressure during in situ experiments. Equation of state fit of the unitcell volume data obtained from high temperature or pressure investigations can provide thermal expansion coefficient, bulk modulus etc., which in turn grant basis for the validation of theoretical models. Different crystal structures of metal hydrides are 
associated with different degrees of iconicity due to increase in coordination number with compression [46]. A comparison of change of molar volume of metal with metal hydride as a function of pressure can be used to determine compressibility of hydrogen in the host [47].

The crystal structure data for alkali and alkali-earth metal hydrides are tabulated in Table 2.4. From Tables 2.2 and 2.4, it is clear that the bulk modulus of alkali and alkali earth metals are lower than that of their hydrides, suggesting a hydrogen induced hardening of these materials. The lower bulk modulus (high compressibility) observed for alkali metal hydrides as compared to the corresponding halides can be due to partial charge transfer between metal and hydrogen atom [48]. X-ray scattering power of hydrides is very weak and hence atomic positions of hydrogen are determined with neutron diffraction or computation. Alkali hydrides show a phase transition at low pressures with 8-13\% volume change and the transition pressure increases with radius of corresponding metal ion [49]. Addition of elements of low atomic weight such as $\mathrm{Si}, \mathrm{Al}$, $\mathrm{B}, \mathrm{C}, \mathrm{N}, \mathrm{P}, \mathrm{S}$ etc., or an appropriate hydride at desirable stochiometry could destabilize metal hydrides without reducing much storage capacities [50].

Complex hydrides show a number of phase transitions with pressure and temperature which are summarized in Tables 2.5 and 2.6. The transition pressure is found to be independent of size of metal atoms in complex hydrides unlike the metal hydrides. Alanates and borohydrides have high compressibility with the bulk modulus range 5- 31 GPa, which suggest possible destabilization under pressure. Except $\mathrm{LiAlH}_{4}$ (monoclinic) and $\mathrm{NaAlH}_{4}$ (tetragonal), alkali alanates are of orthorhombic symmetry. Similar to the 
metals, ambient phase of alkali borohydrides exist in cubic symmetry except $\mathrm{LiBH}_{4}$ which has an orthorhombic structure.

Table 2. 4: A comparison of transition pressure, temperature, structure and bulk modulus data of alkali and alkali- earth metal hydrides. The data marked * is from computation results and others are experimental values.

\begin{tabular}{|c|c|c|c|c|c|}
\hline Hydride & P (GPa) & $\mathbf{T}\left({ }^{\circ} \mathbf{C}\right)$ & Structure/Space group [ref.] & $\mathrm{K}_{\mathbf{0}}(\mathrm{GPa})$ & $\mathbf{K}_{0}^{\prime}$ \\
\hline $\mathrm{LiH}$ & $\begin{array}{l}0-660 \\
>660^{*}\end{array}$ & $\begin{array}{l}25 \\
25\end{array}$ & $\begin{array}{l}\text { Cubic/ } \mathrm{Fm}^{-} 3 \mathrm{~m}[51,52] \\
\text { Cubic/ } \mathrm{Pm}^{-} 3 \mathrm{~m}[53]\end{array}$ & $\begin{array}{l}34.7 \\
31 *\end{array}$ & 0.016 \\
\hline $\mathrm{NaH}$ & $\begin{array}{l}0-29 \\
>29\end{array}$ & $\begin{array}{l}25 \\
25\end{array}$ & $\begin{array}{l}\text { Cubic/ } \mathrm{Fm}^{-} 3 \mathrm{~m}[48] \\
\text { Cubic/ } \mathrm{Pm}^{-} 3 \mathrm{~m}\end{array}$ & $\begin{array}{l}19.4 \\
28\end{array}$ & $\begin{array}{l}4.4 \\
4.3\end{array}$ \\
\hline $\mathrm{KH}$ & $\begin{array}{l}0-4 \\
>4 \\
>176 *\end{array}$ & $\begin{array}{l}25 \\
25 \\
25\end{array}$ & $\begin{array}{l}\text { Cubic/ } \mathrm{Fm}^{-3} \mathrm{~m}[54] \\
\text { Cubic/ } \mathrm{Pm}^{-3} \mathrm{~m} \\
\text { Orthorhombic/cmcm [41] }\end{array}$ & $\begin{array}{l}15.6 \\
28.5\end{array}$ & $\begin{array}{l}4 \\
4\end{array}$ \\
\hline $\mathrm{RbH}$ & $\begin{array}{l}0-2.2 \\
>2.2 \\
>35 *\end{array}$ & $\begin{array}{l}25 \\
25 \\
25\end{array}$ & $\begin{array}{l}\text { Cubic/ } \mathrm{Fm}^{-3} \mathrm{~m}[55] \\
\text { Cubic/ } \mathrm{Pm}^{-} 3 \mathrm{~m} \\
\text { Orthorhombic/cmcm [41] }\end{array}$ & $\begin{array}{l}10 \\
18.4\end{array}$ & $\begin{array}{l}3.9 \\
3.9\end{array}$ \\
\hline $\mathrm{CsH}$ & $\begin{array}{l}0-0.83 \\
1.2-17.5 \\
>17.5\end{array}$ & $\begin{array}{l}25 \\
25 \\
25\end{array}$ & $\begin{array}{l}\text { Cubic/ Fm }{ }^{-3} \text { [56] } \\
\text { Cubic/ } \mathrm{Pm}^{-} 3 \mathrm{~m}[57] \\
\text { Orthorhombic/ cmcm [46] }\end{array}$ & $\begin{array}{l}8,7.6 \\
14.2,22.3 \\
-\end{array}$ & $\begin{array}{l}4 \\
4,4.8 \\
-\end{array}$ \\
\hline $\mathrm{BeH}_{2}$ & & 25 & $\begin{array}{l}\text { Orthorhombic/ Ibam [58] } \\
\text { Amorphous [59] }\end{array}$ & $\begin{array}{l}24.7 \\
14.2\end{array}$ & $\begin{array}{l}3.9 \\
5.3\end{array}$ \\
\hline $\mathrm{MgH}_{2}$ & $\begin{array}{l}0-5.5 \\
5.5-9.5 \\
9.3-10.4 \\
>10 \\
0 \\
2\end{array}$ & $\begin{array}{l}25 \\
25 \\
25 \\
25 \\
>300 \\
>800\end{array}$ & $\begin{array}{l}\text { Tetragonal/ } \mathrm{P} 4_{2} / \mathrm{mnm}[60] \\
\text { Orthorhombic/ Pbcn } \\
\text { Cubic/ } \mathrm{Pa}^{-} 3 \\
\text { Orthorhombic/ Pbca } \\
\text { Tetragonal [60] } \\
\text { Orthorhombic }\end{array}$ & $\begin{array}{l}45 \\
44.03 \\
47.41 \\
49.83\end{array}$ & $\begin{array}{l}3.35 \\
3.49 \\
3.17 \\
3.49\end{array}$ \\
\hline $\mathrm{CaH}_{2}$ & $\begin{array}{l}0-16 \\
15.5-21 \\
>138 *\end{array}$ & $\begin{array}{l}25 \\
25 \\
25\end{array}$ & $\begin{array}{l}\text { Orthorhombic/ Pnma }[61,62] \\
\text { Hexagonal/ } \mathrm{P}_{3} / \mathrm{mmc}[62,63] \\
\text { Hexagonal/ } \mathrm{P}_{3} / \mathrm{mmm}[64]\end{array}$ & $\begin{array}{l}190 *, 40.7 \\
63.6\end{array}$ & $\begin{array}{l}4.5 \\
3.24\end{array}$ \\
\hline $\mathrm{SrH}_{2}$ & $\begin{array}{l}0-8.3 \\
8.3-113 \\
>113\end{array}$ & $\begin{array}{l}25 \\
25 \\
25\end{array}$ & $\begin{array}{l}\text { Orthorhombic/ Pnma [65] } \\
\text { Hexagonal/ } \mathrm{P}_{3} / \mathrm{mmc} \\
\text { Hexagonal/ } \mathrm{P}_{3} / \mathrm{mmm}\end{array}$ & $\begin{array}{l}57,41.4 * \\
75,42.3 * \\
39 *\end{array}$ & $\begin{array}{l}3.13 \\
3.87 \\
3.92\end{array}$ \\
\hline $\mathrm{BaH}_{2}$ & $\begin{array}{l}0-2.5 \\
2.5-50 \\
>50\end{array}$ & $\begin{array}{l}25 \\
25 \\
25\end{array}$ & $\begin{array}{l}\text { Orthorhombic/Pnma }[66,67] \\
\text { Hexagonal/ } \mathrm{P}_{3} / \mathrm{mmc}[68] \\
\text { Hexagonal/? }\end{array}$ & $\begin{array}{l}31.2 *, 24 \\
34.7 *\end{array}$ & $\begin{array}{l}3.2 \\
3.6\end{array}$ \\
\hline
\end{tabular}


Table 2. 5: A comparison of transition pressure, temperature, structure and bulk modulus data of alkali and alkali- earth alanates. The data marked * is from computation results and others are experimental values.

\begin{tabular}{|c|c|c|c|c|c|}
\hline Hydride & P (GPa) & $\mathbf{T}\left({ }^{\circ} \mathbf{C}\right)$ & Structure/ space group [ref.] & $K_{0}(G P a)$ & $\mathbf{K}_{\mathbf{0}}^{\prime}$ \\
\hline $\mathrm{LiAlH}_{4}$ & $\begin{array}{l}0-2.16 \\
>3 \\
>34 \\
7 \\
7\end{array}$ & $\begin{array}{l}25 \\
25 \\
25 \\
>300 \\
>500\end{array}$ & $\begin{array}{l}\text { Monoclinic/ P } 2_{1} / \mathrm{c}[69,70] \\
\text { Tetragonal/ I4 } 4_{1} / \mathrm{a}[69] \\
\text { Orthorhombic/ Pnma }[71] \\
\text { Tetragonal } \\
\text { Orthorhombic/Pnma }[72]\end{array}$ & $\begin{array}{l}13.87,12.9 * \\
25.64 * \\
14.25 *\end{array}$ & $\begin{array}{l}4,4.1 \\
4.35 \\
4.85\end{array}$ \\
\hline $\mathrm{NaAlH}_{4}$ & $\begin{array}{l}0-14 \\
>6.4 \\
>14\end{array}$ & $\begin{array}{l}25 \\
25 \\
25\end{array}$ & $\begin{array}{l}\text { Tetragonal/ } \mathrm{I} 4_{1} / \mathrm{a}[73] \\
\text { Orthorhombic/ } \mathrm{Cmc} 2_{1} *[74] \\
\text { Monoclinic/ } \mathrm{P} 2_{1} / \mathrm{c}\end{array}$ & 27 & 5 \\
\hline $\mathrm{KAlH}_{4}$ & $>0$ & 25 & Orthorhombic/ Pnma $[75,76]$ & $10.34 *$ & 4.61 \\
\hline $\mathrm{RbAlH}_{4}$ & $\begin{array}{l}>0 \\
>\mathrm{xx}\end{array}$ & $\begin{array}{l}25 \\
25\end{array}$ & $\begin{array}{l}\text { Orthorhombic/ Pnma [76] } \\
\text { Orthorhombic/ } \mathrm{Cmc2} 2_{1}\end{array}$ & $\begin{array}{l}9.2 * \\
14.1 *\end{array}$ & $\begin{array}{l}5.2 \\
3.5\end{array}$ \\
\hline $\mathrm{CsAlH}_{4}$ & $\begin{array}{l}>0 \\
>\mathrm{x} \\
>\mathrm{xx}\end{array}$ & $\begin{array}{l}25 \\
25 \\
25\end{array}$ & $\begin{array}{l}\text { Orthorhombic/ Pnma [76] } \\
\text { Tetragonal/ I4 } 1 / \text { a [26] } \\
\text { Orthorhombic/ Cmc } 2_{1}\end{array}$ & $\begin{array}{l}8.6 *, 8.22 * \\
9.5 * \\
9.1 *\end{array}$ & $\begin{array}{l}4.6,5.2 * \\
5.1 \\
4.9\end{array}$ \\
\hline $\operatorname{Mg}\left(\mathrm{AlH}_{4}\right)_{2}$ & $\begin{array}{l}0-0.67 \\
0.67-10.3 \\
>10.3\end{array}$ & $\begin{array}{l}25 \\
25 \\
25\end{array}$ & $\begin{array}{l}\text { Trigonal/ } \mathrm{P}^{-} 3 \mathrm{~m} 1[77,78] \\
\text { Monoclinic/ } \mathrm{C} 2 / \mathrm{m} \\
\text { Orthorhombic/ Pbca }\end{array}$ & $\begin{array}{l}10.9 *, 9.2 * \\
6.8^{*} \\
34.7 *\end{array}$ & 5.7 \\
\hline $\mathrm{Ca}\left(\mathrm{AlH}_{4}\right)_{2}$ & $>0$ & 25 & Orthorhombic/ Pbca* $[33]$ & & \\
\hline
\end{tabular}

High pressure phase transitions of alkali-metal borohydrides are found to be firstorder and fully reversible. Therefore, it is interesting to study structural behavior of metal borohydrides under various conditions of pressures and temperatures. $\mathrm{MBH}_{4}(\mathrm{M}=\mathrm{Na}, \mathrm{K}$, $\mathrm{Rb}, \mathrm{Cs}$ ) compounds show a similar structural phase transition with respect to temperature. At high temperature they crystallize into NaCl-type structures, in which $\mathrm{BH}_{4}^{-}$is octahedrally surrounded by $\mathrm{M}^{+}$. The $\mathrm{H}$ atoms in a $\mathrm{BH}_{4}{ }^{-}$unit are tetrahedrally oriented about $\mathrm{B}$ and along all cube diagonals. This gives a random distribution of $\mathrm{BH}_{4}^{-}$ tetrahedron in two different configurations. 
Table 2. 6: A comparison of transition pressure, temperature, structure and bulk modulus of alkali and alkali- earth borohydrides. The data marked * is from computation results and others are experimental values.

\begin{tabular}{|c|c|c|c|c|c|}
\hline Hydride & P (GPa) & $\mathbf{T}\left({ }^{\circ} \mathbf{C}\right)$ & Structure/ Space group [ref.] & $\mathrm{K}_{0}(\mathrm{GPa})$ & $\mathbf{K}_{\mathbf{0}}^{\prime}$ \\
\hline $\mathrm{LiBH}_{4}$ & $\begin{array}{l}0-0.8 \\
1.1-10 \\
>2.4 \\
>10 \\
0 \\
0\end{array}$ & $\begin{array}{l}25 \\
25 \\
25 \\
25 \\
111 \\
-48\end{array}$ & $\begin{array}{l}\text { Orthorhombic/Pnma [79-81] } \\
\text { Monoclinic/ } \mathrm{P} 2_{1} / \mathrm{c}[79] \\
\text { Tetragonal/Ama }[82] \\
\text { Cubic/ Fm }-3 \mathrm{~m}[79] \\
\text { Hexagonal/ } \mathrm{P} 6_{3} \mathrm{mc}[83] \\
\text { Orthorhombic/ Pnma }[45,82]\end{array}$ & $\begin{array}{l}14.4,15 * \\
23.23 \\
26\end{array}$ & $\begin{array}{l}3.5 \\
3.5 \\
3.5\end{array}$ \\
\hline $\mathrm{NaBH}_{4}$ & $\begin{array}{l}0-6.3 \\
6.3-8.9 \\
8.9-14 \\
>14 \\
0 \\
6.5 \\
9.5\end{array}$ & $\begin{array}{l}25 \\
25 \\
25 \\
25 \\
<-83 \\
>100 \\
>150\end{array}$ & $\begin{array}{l}\text { Cubic/ Fm }-3 \mathrm{~m}[84,85] \\
\text { Tetragonal/ } \mathrm{P}^{-} 42_{1} \mathrm{c} \\
\text { Orthorhombic/ Pnma } \\
\text { Monoclinic/ } \mathrm{P} 2_{1} / \mathrm{c}[86] \\
\text { Tetragonal / } \mathrm{P}^{-} 42_{1} \mathrm{c} \\
\text { Cubic/ Fm-3m }[86] \\
\text { Tetragonal / } \mathrm{P}^{-} 42_{1} \mathrm{c}\end{array}$ & $\begin{array}{l}19.9,18.76 \\
30.6^{*} \\
31.1\end{array}$ & $\begin{array}{l}3.5,3.48 \\
3.9\end{array}$ \\
\hline $\mathrm{KBH}_{4}$ & $\begin{array}{l}0-3.8 \\
3.8-6.8 \\
>6.8 \\
0\end{array}$ & $\begin{array}{l}25 \\
25 \\
25 \\
-197\end{array}$ & $\begin{array}{l}\text { Cubic/ } \mathrm{Fm}^{-} 3 \mathrm{~m}[87] \\
\text { Tetragonal/ } \mathrm{P}^{-} 42_{1} \mathrm{c} \\
\text { Orthorhombic/ Pnma } \\
\text { Tetragonal/ } \mathrm{P}_{2} / \mathrm{nmc}[88,89]\end{array}$ & 16.8 & 4 \\
\hline $\mathrm{RbBH}_{4}$ & $\begin{array}{l}0-2.5 \\
2.5-8 \\
>8 \\
0\end{array}$ & $\begin{array}{l}25 \\
25 \\
25 \\
-229\end{array}$ & $\begin{array}{l}\text { Cubic/ } \mathrm{Fm}^{-} 3 \mathrm{~m}[90] \\
\text { Orthorhombic/ Pnma } \\
\text { Monoclinic/ } \mathrm{P} 2_{1} / \mathrm{c} \\
\text { Tetragonal }[88]\end{array}$ & 14.5 & 4 \\
\hline $\mathrm{CsBH}_{4}$ & $\begin{array}{l}0 \\
>\mathrm{x} \\
>\mathrm{xx} \\
0\end{array}$ & $\begin{array}{l}25 \\
25 \\
25 \\
-246\end{array}$ & $\begin{array}{l}\text { Cubic/ } \mathrm{Fm}^{-} 3 \mathrm{~m}[91] \\
\text { Tetragonal/ } \mathrm{P}^{-} 42_{1} \mathrm{c} \\
\text { Orthorhombic/ } 4_{2} / \mathrm{nmc} \\
\text { Tetragonal }[88]\end{array}$ & $\begin{array}{l}5.2 * \\
10.4 * \\
5.3 *\end{array}$ & $\begin{array}{l}5.9 \\
4.9 \\
5.9\end{array}$ \\
\hline $\mathrm{Be}\left(\mathrm{BH}_{4}\right)_{2}$ & 0 & 25 & Tetragonal/ I4 ${ }_{1} \mathrm{~cd}[36,92]$ & & \\
\hline $\mathrm{Mg}\left(\mathrm{BH}_{4}\right)_{2}$ & $\begin{array}{l}0 \\
0-2.5 \\
2.5-14 \\
>14 \\
0\end{array}$ & $\begin{array}{l}25 \\
25 \\
25 \\
25 \\
>180\end{array}$ & $\begin{array}{l}\text { Orthorhombic/Pmc } 2_{1}[78] \\
\text { Hexagonal/ } \mathrm{P} 6_{1} 22[93] \\
\text { Hexagonal/ } \mathrm{P}_{3}[94] \\
\text { ? unidentified } \\
\text { Orthorhombic/Fddd [95] }\end{array}$ & $\begin{array}{l}7.82 * \\
10.16\end{array}$ & $\begin{array}{l}5.2 \\
4\end{array}$ \\
\hline $\mathrm{Ca}\left(\mathrm{BH}_{4}\right)_{2}$ & $\begin{array}{l}>0 \\
0-10.2 \\
0 \\
0 \\
0 \\
0\end{array}$ & $\begin{array}{l}25 \\
25 \\
<-182 \\
>32 \\
>222 \\
>207\end{array}$ & $\begin{array}{l}\text { Orthorhombic/ F2dd }[96,97] \\
\text { Tetragonal/ P4 } 4 \text { /m } \\
\text { Orthorhombic/F2dd [96] } \\
\text { Tetragonal / } \mathrm{P}^{-} 4 \\
\text { Tetragonal / I } 4_{2} \mathrm{~d} \\
\text { Tetragonal / } \mathrm{P} 4_{2} / \mathrm{m}[98]\end{array}$ & 22.95 & 2.63 \\
\hline
\end{tabular}


In the case of $\mathrm{MBH}_{4}$ (where $\mathrm{M}$ is an alkali metal), as the cation size increase from Li to $\mathrm{Cs}$ the unit cell expands and $\mathrm{H}-\mathrm{H}$ bond length increases. Therefore the $\mathrm{H}-\mathrm{H}$ repulsion will be weaker in heavier $\mathrm{MBH}_{4}$ which results in reduction of cubic to tetragonal transition temperature or increase in corresponding transition pressure [99]. The high pressure structural behavior of alkali borohydrides is widely investigated. Nevertheless, the high pressure structural data of alkali-earth alanates and borohydrides are scarce owing to their high reactivity and difficulty to synthesize.

\subsection{Applications of Hydrides}

Hydrides are important materials both for possible use in transportation and for nuclear industry. The use of metal hydrides and borohydrides as advanced shielding materials in nuclear power plants is well known [100]. Examples are $\mathrm{Mg}\left(\mathrm{BH}_{4}\right)_{2}, \mathrm{TiH}_{2}$ and $\mathrm{ZrH}_{2}$ which can reduce the thickness of the outboard shield by 23,20 and $19 \%$, respectively, compared to the combination of steel and water. Hydrides have a variety of applications as listed below [101].

○ Electrochemical (rechargeable batteries, catalysis, fuel cells)

○ Thermal (heat storage, refrigeration, heat engine/ actuator, heat pumping)

○ Nuclear industry (fuel cladding, neutron shielding and reflection, fuel-moderator)

O Sensors and optical switch

○ Hydrogen processing (purification, isotope separation, compression)

To power a fuel cell, a metal hydride has remarkable advantages in regard to cryogenic or pressure storage tanks as it does not require additional power supply, there is no loss, and hydrogen can be safely supplied at an optimum and constant pressure. 
Main issue in using complex metal hydrides is the difficulty in thermal management during refueling because of the reaction enthalpies involved. The advantage of using a fuel cell system is that the dehydrogenation of complex hydrides does not require water [102]. 


\section{CHAPTER 3. EXPERIMENTAL TECHNIQUES AND DETAILS}

\subsection{Diamond Anvil Cell Technique}

The diamond anvil cell (DAC) is a portable high pressure device which use the force applied to a smaller area to maximize the pressure generated. Table 3.1 summarizes the devices with which ultra high pressures can be applied in the laboratory setting. With the help of external or internal heaters it is possible to study properties of materials under both pressure and temperature in these devices. The DAC technique is based upon the opposed-diamond configuration, in which a sample is placed in the hole of a gasket (metal, rhenium, graphite etc.) inserted between the polished culets of two diamonds. With this configuration, very small force can create extremely large pressures on the sample.

Table 3. 1: High pressure apparatuses and pressure- temperature ranges that can be achieved using each of them.

\begin{tabular}{lll}
\hline Method & $\begin{array}{l}\text { Pressure } \\
\text { P (GPa) }\end{array}$ & $\mathbf{T}\left({ }^{\circ} \mathbf{C}\right)$ \\
\hline Gas apparatus & 3 & 1500 \\
Piston- cylinder & 5 & 2000 \\
Bridgman anvils & 20 & 2000 \\
Belt apparatus & 20 & 2000 \\
Multi anvil press & 30 & 2500 \\
DAC & 200 & 2000 (resistive) \\
& & 4000 (laser) \\
Shock wave & 800 & 5000 \\
\hline
\end{tabular}


Because the diamonds are transparent to most part of the electromagnetic spectrum (X-rays, gamma rays, UV, IR, visible light etc.), the sample may be examined in situ by spectroscopic and diffraction techniques. Different types of DACs generally used are Merril- Baset, P series, Mao- Bell, compact cylinder cell, and four post high pressure cells. Figure 3.1 shows a loaded Mao- Bell type DAC and a schematic of the sample chamber in DAC.

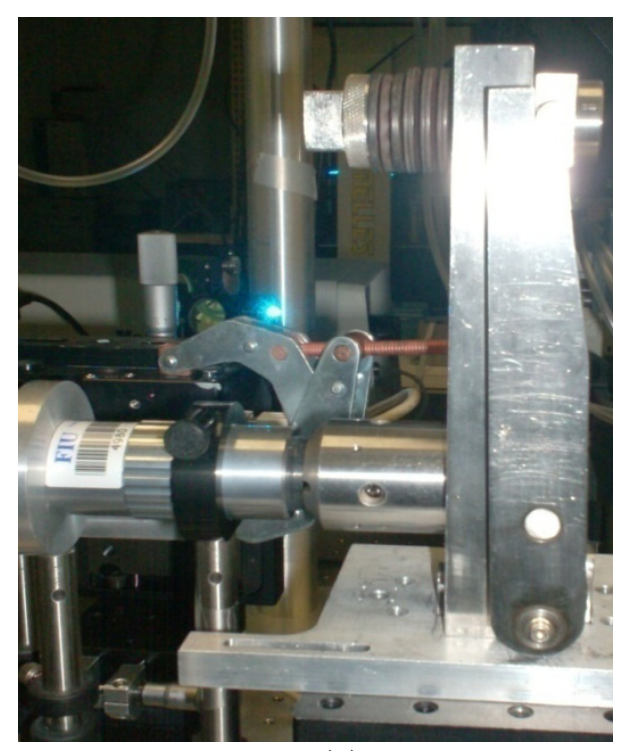

(a)

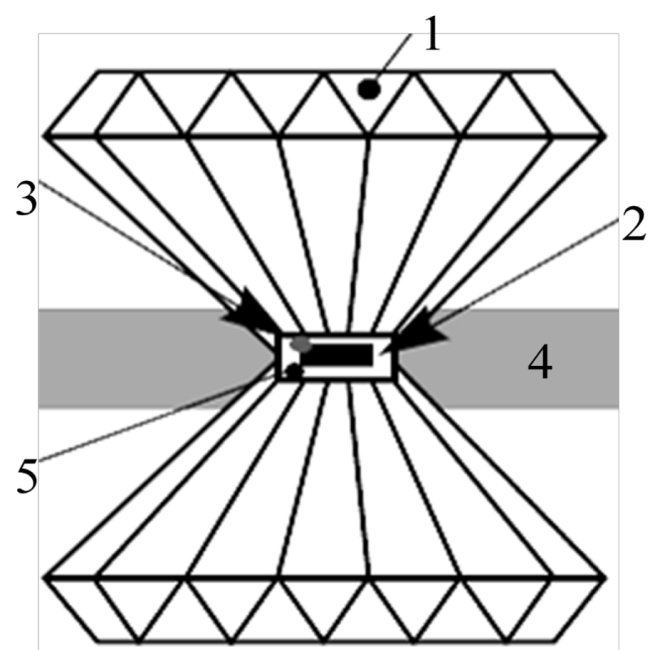

(b)

Figure 3. 1: a) A loaded Mao- Bell type DAC; b) schematic of the sample chamber in a diamond anvil cell, 1- diamond, 2- gasket hole, 3- sample, 4- gasket, 5- ruby chip.

Compressive strength, hardness, and transparency to a wide electromagnetic spectrum are the main properties of diamond that make it a good choice for anvils. Two of the most important properties of a material are crystal structure and lattice parameters that can be used for determining basic thermodynamic quantities, such as the effect of pressure and temperature on molar volume. X-ray diffraction using DAC is the best tool 
for such studies as the building block of diamond, the $\mathrm{C}$ atoms, has low atomic number and hence low absorption. DACs can also be used for a number of investigations using IR, UV, gamma rays, sound waves etc., because of the extraordinary optical and elastic properties of diamonds [103]. Insertion of a gasket between the diamonds of the DAC helps to encapsulate a hydrostatic pressure medium, also to carry out viscosity measurements, chemical reactions, and structure determinations of molecular species in solution.

\subsubsection{Measurement of pressure in a DAC}

The pressure in the DAC can be measured by an embedded pressure marker or ruby $\left(\mathrm{Cr}^{3+}\right.$ doped $\left.\mathrm{Al}_{2} \mathrm{O}_{3}\right)$. A material of known pressure- volume EoS such as $\mathrm{NaCl}$, $\mathrm{MgO}, \mathrm{Pt}, \mathrm{Au}, \mathrm{W}, \mathrm{Ag}, \mathrm{Cu}$ etc., are generally used as pressure marker. If the pressure marker is mixed with sample, the lattice parameters can be obtained by X-ray diffraction which in turn can be used in 'calibration' software to determine pressure. Ruby pressure scale is a faster method to measure pressure in the DAC experiments. Ruby has a series of spectral bands (R, U, B and Y) in the visible ranges. The high energy lines $U, B$ and $Y$ can be excited by a laser and they relax to $R$ lines. The $R$ lines $\left(R_{1}\right.$ and $R_{2}$ with wave lengths 694.2 and $692.8 \mathrm{~nm}$, respectively at ambient conditions) are metastable with long lifetime which shift to higher wave length under pressure (Figure 3.2), and relax to ground state by fluorescence. The ruby method is not suitable for measuring pressure at high temperatures, because of the shift and fading away of the fluorescence signal with temperature. Metal pressure markers such as Pt are chemically inert and scatter X-rays efficiently, and the effects of both pressure and temperature on lattice dimensions are 
well known. Another method good for high temperature pressure measurement is fluorescence of a samarium doped compound $\left(\mathrm{SrB}_{4} \mathrm{O}_{7}: \mathrm{Sm}^{2+}\right)$. In the present study, both ruby and $\mathrm{NaCl}$ are used for pressure measurement in high pressure- temperature experiments.

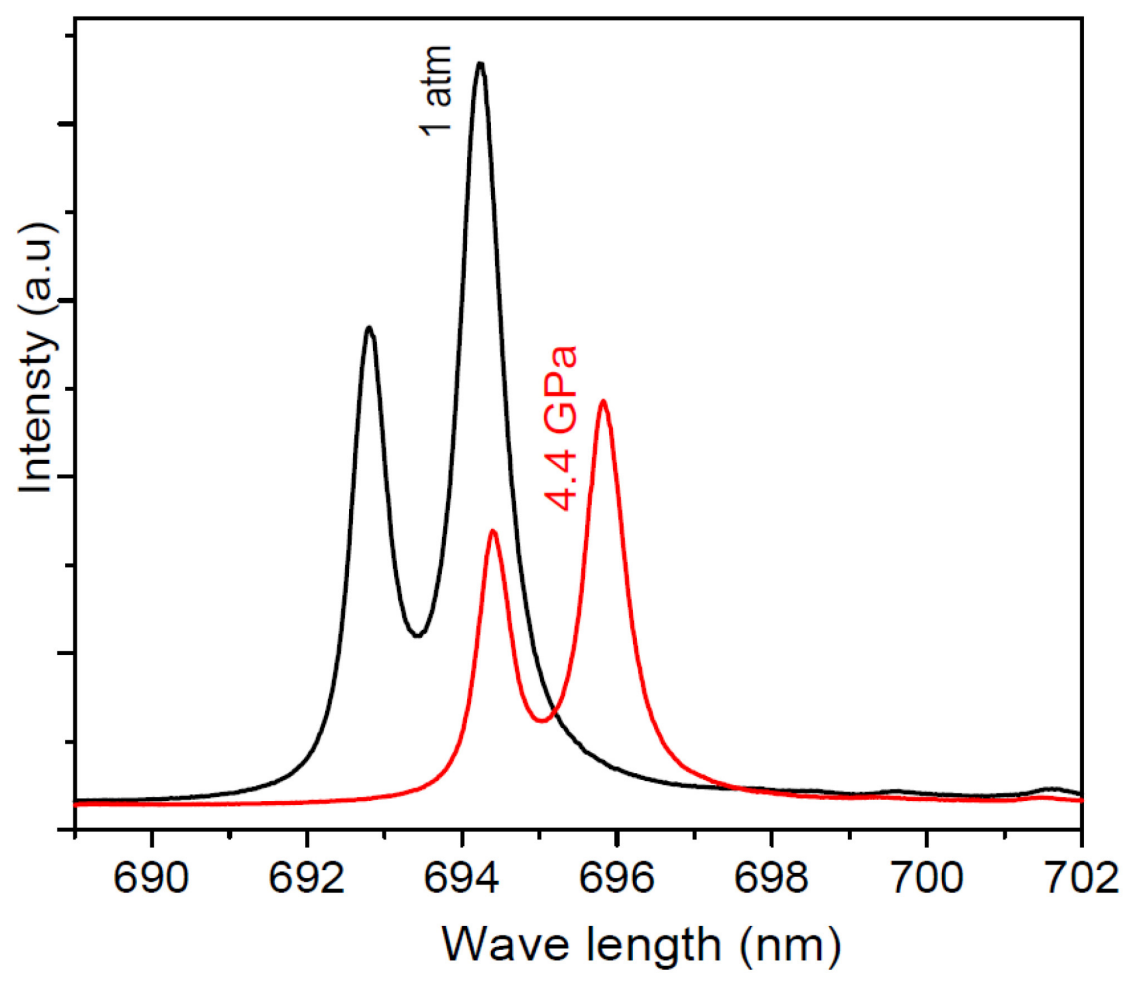

Figure 3. 2: The shift of ruby fluorescent spectrum with the application of a pressure of 4.4 GPa.

Mao et al. [104], carried out the calibration of the pressure shift of $\mathrm{R}_{1}$ emission line of ruby up to $80 \mathrm{GPa}$ under quasi hydrostatic conditions with argon gas as a pressure transmitting medium. The shift of $\mathrm{R}_{1}$ line with pressure was found to be linear with pressure about $0.274 \mathrm{GPa} \AA^{-1}$ and can be fitted to the formula; $\mathrm{P}(\mathrm{GPa})=(\mathrm{A} / \mathrm{B})\left[\left(\lambda / \lambda_{0}\right)^{\mathrm{B}}-1\right]$ where $\mathrm{A}=1904 \mathrm{GPa}, \mathrm{B}=7.665, \lambda$ is the wavelength of $\mathrm{R}_{1}$ line at pressure $\mathrm{P}$ and $\lambda_{0}$ is that 
at ambient conditions. Later, with helium as pressure medium Silvera et al. [105], calibrated the ruby pressure gauge up to $150 \mathrm{GPa}$ with a difference in $\mathrm{A}$ and $\mathrm{B}$ parameters. The pressure can be determined from the reduced equation using change in wave length of $\mathrm{R}_{1}$ line; $\mathrm{P}=\Delta \lambda / 0.365$ where $\mathrm{P}$ is in GPa. Reduction in the X-ray beam divergence and collimation diameter $(\sim 0.15 \mathrm{~mm})$ can be useful to minimize the effect of pressure gradients in the compressed sample. The ruby fluorescence is recorded using a USB based fluorescence spectrometer (Ocean Optics) and the operating softwareOOIBase32.

\subsubsection{Sample loading in the DAC}

All sample handlings were carried out in an argon atmosphere. Mao-Bell type DAC with diamonds of 0.4-0.5 mm culets and stainless steel (T302) gasket of 0.18-0.21 $\mathrm{mm}$ hole was used. Steel gaskets were of thickness $0.27-0.32 \mathrm{~mm}$ and were indented to 0.05-0.07 $\mathrm{mm}$ using the DAC before drilling hole. A motorized electric discharge machine from BETSA is used to drill holes in the gaskets (Figure 3.3). The principle used for drilling is the spark erosion in which a high voltage spark erodes the metallic gasket immersed into a dielectric liquid like isopropanol. For medium and high pressures steel and rhenium gaskets, respectively are good. At high pressures, all materials can develop shear strength leading to non hydrostatic conditions. Fluids like helium, hydrogen, xenon etc., are used to create quasi hydrostatic conditions. For pressures less than $15 \mathrm{GPa}$, a 4:1 methanol: ethanol mixture and silicone oil are found to be effective. In heating experiments, a resistive ring-heater and specially designed thermocouples were employed

around diamond culets. The outer surface of the DAC was cooled by water circulating 
setup or argon gas. To accurately measure the pressure, both $\mathrm{NaCl}$ and ruby chips were added to the sample in the high temperature experiments. Measurements were conducted with and without a pressure transmitting medium (silicone oil or mineral oil) and observed highly non hydrostatic behavior above $12 \mathrm{GPa}$ in both cases because of their solidification under pressure. In the heating experiments no pressure transmitting medium was used. Because of high refractive index of diamonds in DAC, a correction has been carried out on the diffractometer stage or goniometer to obtain accurate sample position.

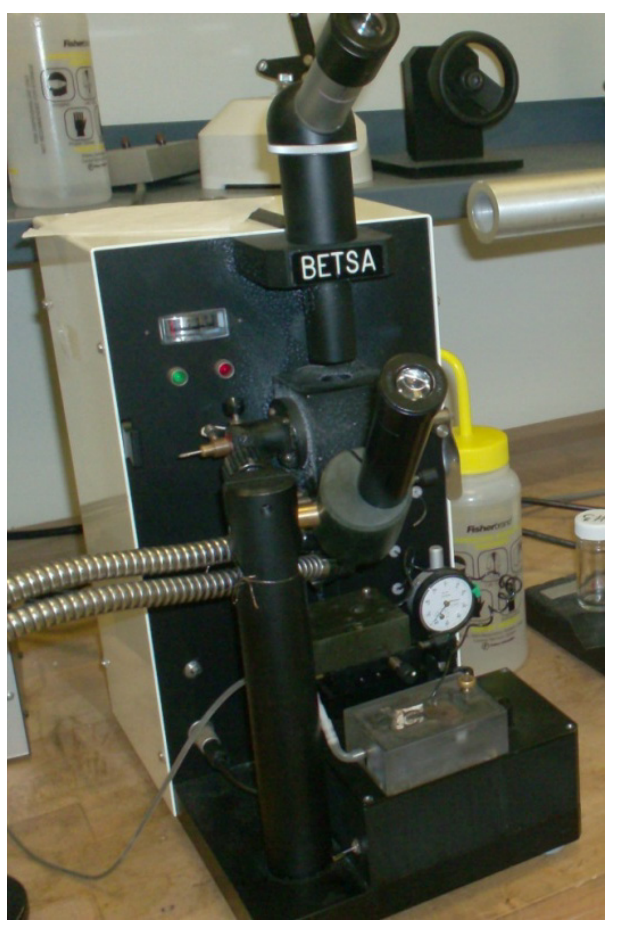

Figure 3. 3: Drilling machine which is employed to drill holes in the gaskets using spark erosion method.

\subsubsection{Resistive heating in a DAC}

For high temperature study under pressure, samples in DAC can be electrically heated with a ring heater placed around the anvils or wrapping resistance wires around 
the diamond supports. A specially designed gasket also can be used as a heater [106]. Resistive heaters can provide temperatures up to $1000{ }^{\circ} \mathrm{C}$. Thermocouple attached to diamond surface is the most satisfactory way to measure temperature because of its high thermal conductivity. In the present study, a ring type heater consisting of pyrophilite $\left(\mathrm{AlSi}_{2} \mathrm{O}_{5} \mathrm{OH}\right)$ disc wounded with nickel-chromium (nichrome) wire of resistance about 6 ohm was placed around diamonds with proper insulation. Specially designed K-type (Ni$\mathrm{Cr} / \mathrm{Ni}-\mathrm{Al}$ ) thermocouple junction was employed close to the diamond culet, which in turn connected to a temperature controller and DC power supply of $\sim 30 \mathrm{~V}$. The thermocouple used is good to measure temperatures in the range -270 to $1260 \circ \mathrm{C}$.

\subsection{X-Ray Diffraction Techniques}

\subsubsection{Powder X-ray diffraction}

Powder X-ray diffraction (XRD) is an efficient tool to determine crystal structure of a material. The X-ray beam may be white having a broad spectrum (Laue) or monochromatic (Bragg) having essentially a single energy. The white beam can be used to produce diffraction patterns by the energy-dispersive (ED) method which analyzes energies (wavelengths) of X-rays scattered in a specific direction. Monochromatic radiation is used for angle dispersive (AD) method which has better resolution. The intensities around each diffraction ring can be integrated using computer processing with improved signal-to-noise ratio and accuracy.

In reflection (Bragg) geometry, data collection is confined within a plane. Only crystallites having reflection planes (hkl) parallel to the specimen surface will contribute to the reflected intensities. In powder or polycrystalline diffraction it is important to have 
a sample with plane surface. To obtain better diffraction, samples should be ground down to particle sizes of few microns and pressed into a sample holder such as quarts capillary. With a 2D diffraction detection in transmission geometry, a large portion of the diffraction rings (Debye ring) can be measured simultaneously. The $2 \mathrm{D}$ pattern can be analyzed after integrating which gives better intensity and statistics for phase identification and quantitative analysis.

Table 3. 2: Different information available from the condition of Debye rings in a $2 \mathrm{D} X-$ ray diffraction experiment.

\begin{tabular}{ll}
\hline Debye diffraction rings & Condition of the sample \\
\hline Continuous & Fine grained \\
Spotty & Coarse grained \\
Narrow & Strain free \\
Broad & Residual stress, small particle size \\
Uniform intensity & Random orientation \\
Non uniform intensity & Preferred orientation \\
\hline
\end{tabular}

In a diffraction pattern, unit cell determine the line positions and atomic positions attribute to the line intensity. Low symmetry crystals like triclinic have more lines in powder XRD pattern than a more symmetric system like cubic, due to introduction of new plane spacing by non uniform distortion. Therefore a new peak at high pressure or temperature experiments implies low to high symmetry transition. And, disappearance of a peak means low to high symmetry transition; for example orthorhombic to tetragonal. Sample orientation has no effect on profile from 2D frame. A 2D diffraction pattern contains more information than the conventional pattern ( $2 \theta$ versus intensity) for applications such as phase identification, percent of crystalline phase, particle size and 
shape, texture and stress as summarized in Table 3.2. In order to get beam center and detector distance accurately, a powder pattern should be acquired with standards like $\mathrm{CeO}_{2}, \mathrm{LaB}_{6}, \mathrm{Si}, \mathrm{Al}$ etc. Lattice parameters as a function of pressure or temperature can be obtained by the Rietveld analysis of the diffraction pattern.

\subsubsection{Synchrotron X-ray source}

Hydrides are light materials with low scattering power. Furthermore, the use of DAC limits the amount of sample we can use. To resolve dynamic structural data, good quality diffraction patterns are required which can be obtained using a synchrotron XRD. A high-energy synchrotron source produces a very intense, small, and well collimated beam of X-rays with high resolution which can be used to study small samples such as in a DAC. Synchrotron X-rays are electromagnetic (EM) radiation generated by accelerating electrons. The high-energy electron beam is directed into auxiliary components such as bending magnets and insertion devices (undulaters or wigglers) in storage rings and free electron lasers. This generates strong magnetic fields perpendicular to the beam which helps to convert the high-energy of electron into an EM radiation. Finally, the radiation is projected at a tangent to the electron storage ring and captured by beam lines. The beam lines can be at the bending magnets located at the corners, or insertion devices placed in the straight sections of the storage ring and both types have different spectrum and energy. A beam line includes optical devices (slits, attenuators, crystal monochromators, and mirrors) which control the bandwidth, photon flux, beam dimensions, focus, and collimation of the X-ray beam. At the end of the beam line is an experimental station, where the samples are placed in the line of the radiation and 
detectors (image plate or charge-coupled device) are positioned to measure the resulting diffraction. Figure 3.4 shows a schematic diagram of the X-ray diffractometer having transmission (Laue) geometry. Both white and monochromatic beams are valuable for diamond anvil cell studies. To collect a reasonably good diffraction pattern from DAC when using light hydrides, longer exposure time is required. In such cases, use of large sized image plates is preferred to get better resolution and to avoid dark current accumulation.

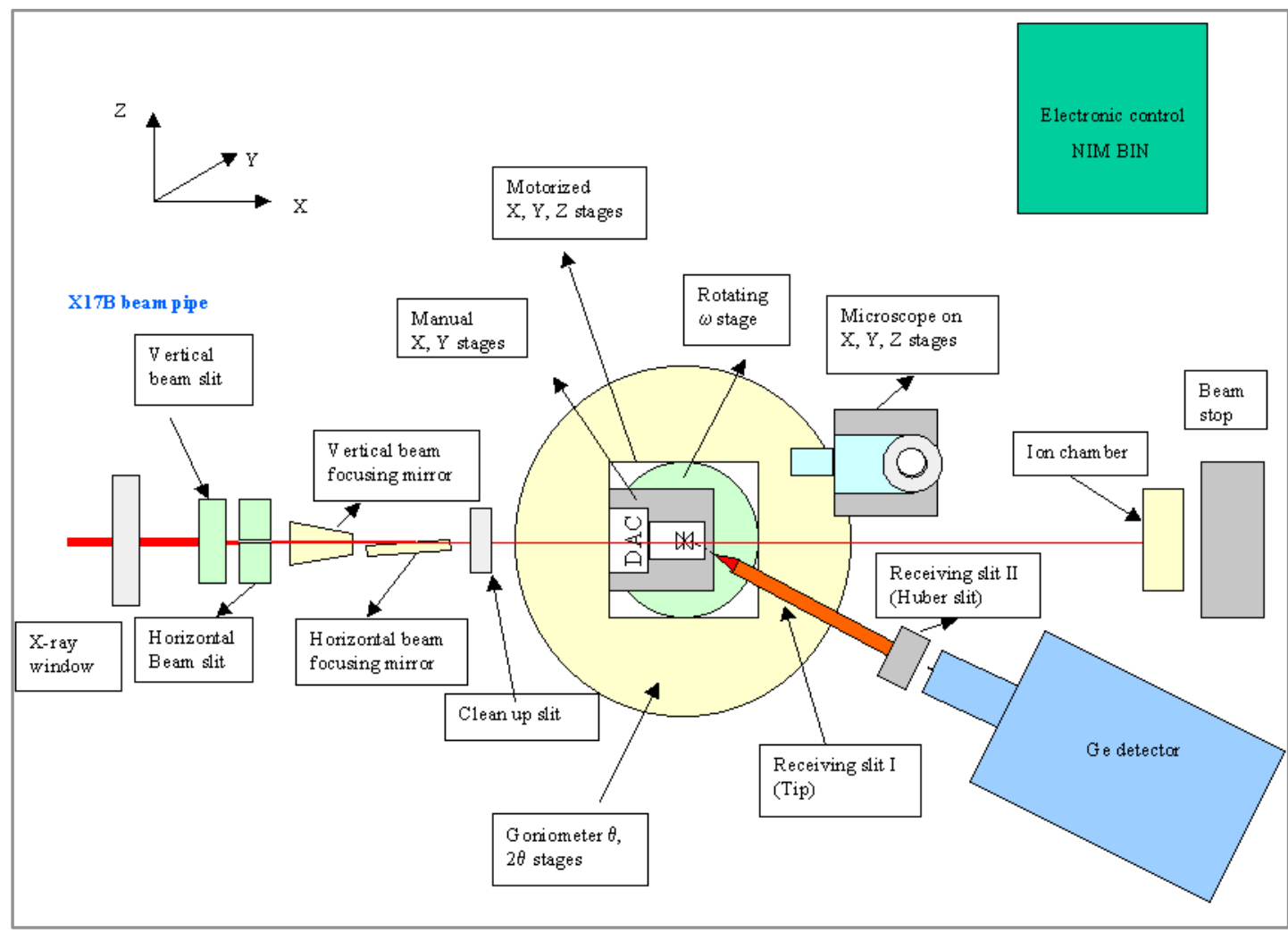

Figure 3. 4: Schematics of an X-ray diffractometer with transmission geometry; adapted from X17C hutch at NSLS [107]. 
The high pressure and temperature XRD measurements were mainly conducted at different beam lines of national synchrotron facilities. The diffracted X-rays were collected using Mar345 image plate detector. The display and control soft wares were based on MEDM, Java, and IDL. The beam resolution was $10^{-4}$. Calibration of the sample position and detector distances was carried out using $\mathrm{CeO}_{2}$ standard. The synchrotron facilities used were;

- Sector 16-IDB, HPCAT, Advanced Photon Source of Argonne National Laboratory using X-rays of wavelength $\sim 0.3681 \AA$ (Figure 3.5). Source: Undulator.

- X17C beam line at NSLS, Brookhaven National Laboratory with facilitating radiation of $\lambda=0.4066 \AA$. Source: Superconducting wiggler.

- Station B2 of Cornell high energy synchrotron source (CHESS) in Cornell University with facilitating radiation of wavelength, $\lambda=0.49594 \AA$. Source: Bending magnet.

- X17B2 beam line of National Synchrotron Light Source in Brookhaven National Laboratory (energy- dispersive, $2 \theta=6.4762^{\circ}$ ). Source: Superconducting wiggler.

\subsubsection{Rotating anode $\mathrm{X}$-ray generator}

Electrons emitted from a heated cathode is accelerated by an electric field and collide with the anode target metals like tungsten, molybdenum or copper, and accelerate other electrons, ions and nuclei within the anode material. Most of the kinetic energy contained in the electron beam is converted to heat which is dissipated via a heat sink, and about $1 \%$ of the energy generated in the accelerated particles is emitted, usually perpendicular to the path of the electron beam, as X-rays. At the electron focal spot, Xray photons are emitted in all directions from the target surface, the highest intensity 
being around 60 to 90 degrees from the beam due to the angle of the anode target to the approaching X-ray photons. The generated beam is directed out the X-ray tube and projected on sample material which will be partially diffracted. The resulting diffraction pattern of the radiation is then detected by a medium including rare earth screens (which surround photographic film), semiconductor detectors, charge-coupled devices (CCDs) or X-ray image intensifiers. The advantage of rotating anode over stationary anode design is an effective increase in the area of anode target bombarded by accelerated electrons and hence the added brilliance. Figure 3.6 shows X-ray diffractometer at CeSMEC which consists of a rotating anode generator.

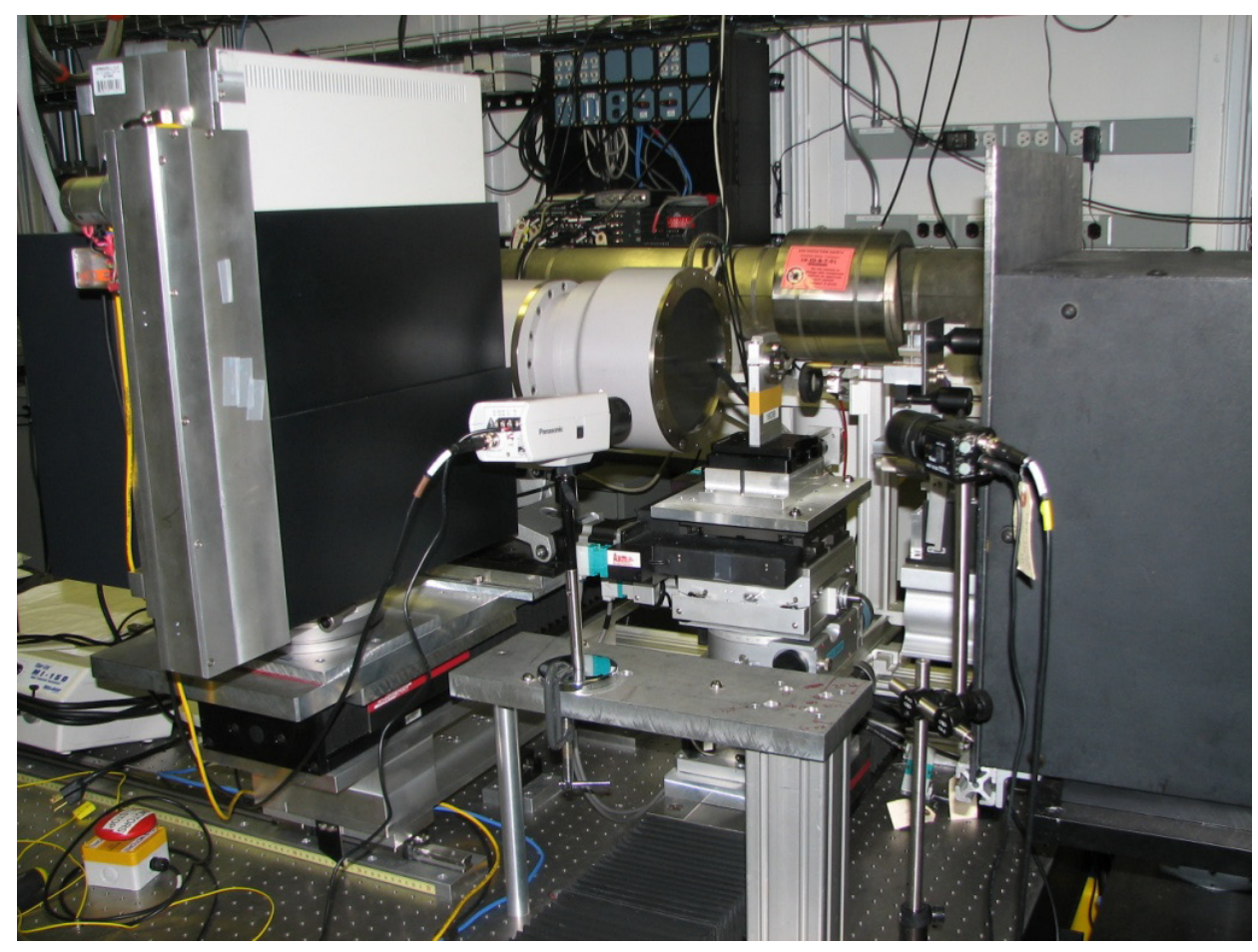

Figure 3. 5: The X-ray diffractometer stage at the beam line 16 IDB- HPCAT of Argonne National Laboratory. 
A portion of the X- ray diffraction studies were carried out using Bruker- GADDS/D8 X-ray system with MacSci rotating anode of molybdenum $(\lambda \sim 0.71073 \AA)$ and Apex Smart CCD detector. For heating experiments at ambient pressure, sample was loaded in quartz capillary and kept under vacuum overnight before sealing with Ar gas to avoid oxidation of the sample. A home- made heater coupled with temperature controller was used to heat the sample for in situ XRD experiments up to $500{ }^{\circ} \mathrm{C}$. Temperature was calibrated from the thermal expansion of $\mathrm{NaCl}$. The sample position was calibrated using $\mathrm{LaB}_{6}$

\subsubsection{Indexing of X-ray diffraction patterns}

From angular position of diffraction lines, the unit cell parameters are deduced assuming crystal system from seven possibilities, and then assigning Miller indices (hkl) to each reflection. Number of atoms per unit cell can be computed from unit cell parameters, chemical composition and measured intensity. Position of an atom in the unit cell is determined from relative intensities of diffraction lines. In powder methods the wavelength used should not be shorter than K-absorption edge of the sample material to avoid fluorescence. Shorter the wavelength, smaller will be the theta and therefore number of lines in the pattern will be more.

There are a number of factors affecting relative intensity of diffraction lines in a powder XRD viz; polarization factor, structure factor, multiplicity factor, Lorentz factor, absorption factor and temperature factor. Background radiation is caused by fluorescence from sample because of diffraction of continuous spectrum in the incident beam. The main causes are, diffuse scattering from sample like incoherent (Compton) scattering 
when the atomic size is small, coherent scattering due to temperature in soft materials of low melting point, diffuse scattering due to crystal imperfections, diffraction and scattering from collimator, gasket, binder etc.

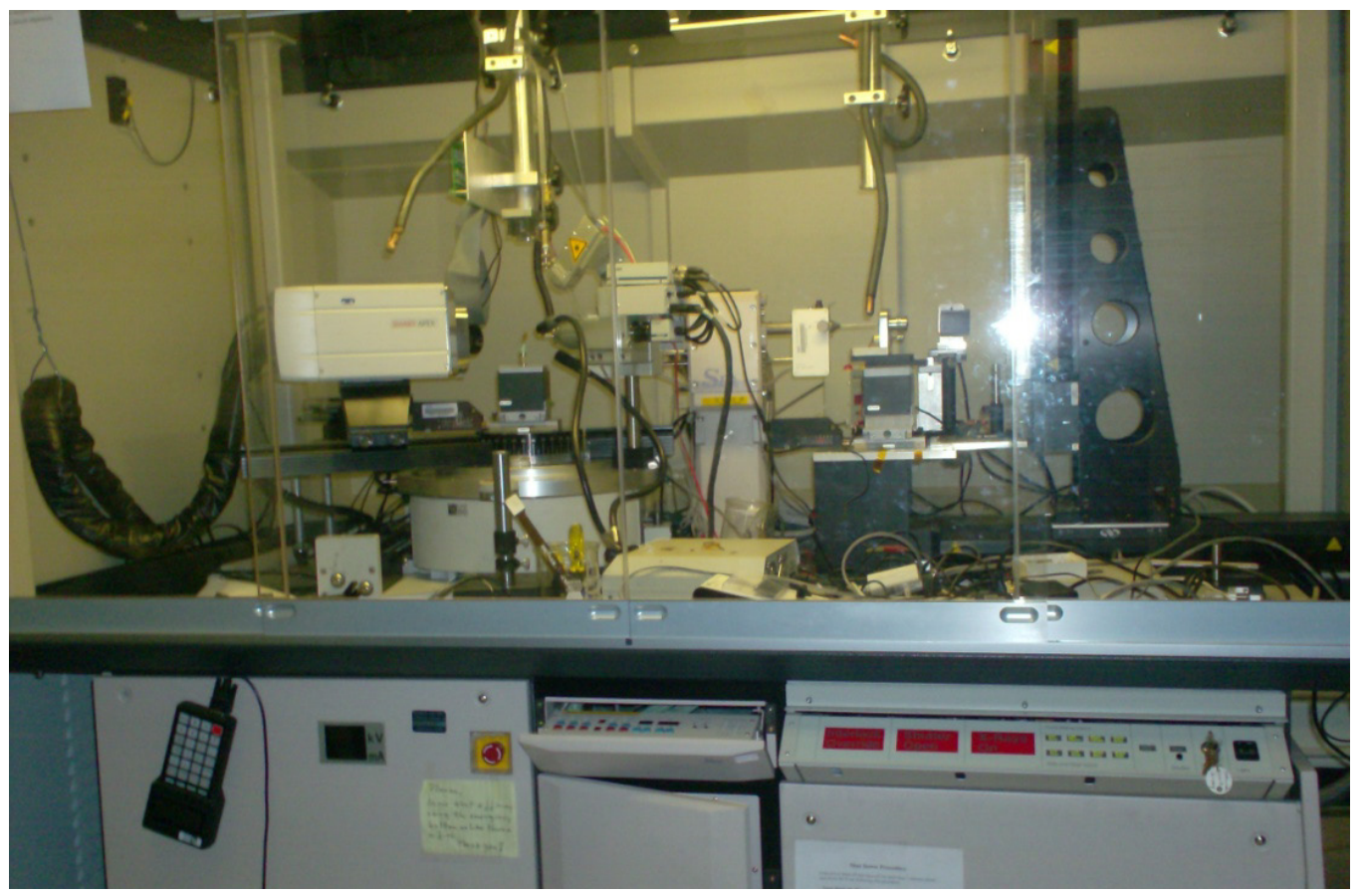

Figure 3. 6: A Bruker- GADDS/D 8 X-ray system with molybdenum rotating anode Xray generator at CeSMEC.

\subsection{Raman Spectroscopy}

Raman spectroscopy experiments were carried out with an argon ion $\left(\mathrm{Ar}^{+}\right)$laser system (Spectra Physics, model 177G02) of $\lambda=514.5 \mathrm{~nm}$. The Raman spectroscopy instrument used to conduct experiments which is equipped with a ruby fluorescence spectrometer is shown in Figure 3.7. Backscattered Raman spectra were collected by high throughput holographic imaging spectrograph (Kaiser Optical Systems, model HoloSpec $f / 1.8 i$ ) with volume transmission grating, holographic notch filter and thermoelectrically 
cooled CCD detector (Andor Technology). The average spot size of the laser beam used is $\sim 0.005 \mathrm{~mm}$. The Raman spectrometer system has a spectral resolution of $4 \mathrm{~cm}^{-1}$ and the spectra were collected at an exposure of 600-1800s depending on the sample.

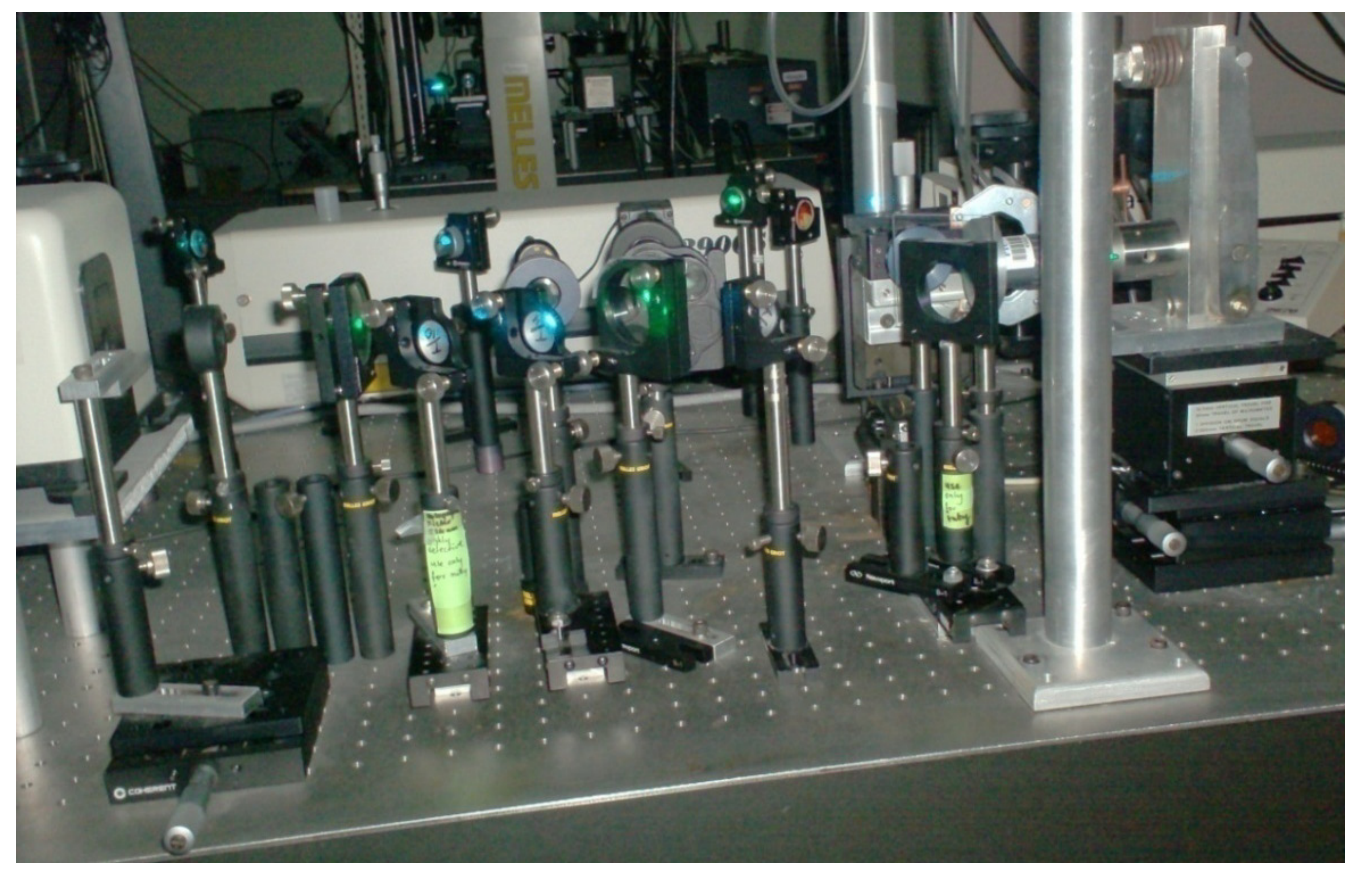

Figure 3. 7: Raman spectroscopy setup with in situ ruby pressure measurement system at CeSMEC.

In Raman spectroscopy a light of known frequency and polarization is scattered from the sample which is analyzed for the frequency shift which is an intrinsic property of the sample. The classical vibrational frequency of a diatomic molecule is; $v=$ $(1 / 2 \pi \mathrm{c})(\mathrm{k} / \mu)^{1 / 2}$, where $\mathrm{c}$ is the velocity of light, $\mathrm{k}$ is the force constant and $\mu$ is the reduced mass of the constituting atoms. The Raman shift from incident laser frequency $\left(v_{0}\right)$ is termed as Stokes $\left(v_{0}-v_{\mathrm{m}}\right)$ and anti- Stokes $\left(v_{0}+v_{\mathrm{m}}\right)$ lines. The vibrational patterns of atoms and the polarization dependence of scattering are determined by the equilibrium 
structure of the material through the rules of group theory. The Raman shift is the change in the frequency of the scattered light from the laser frequency which implies that energy is deposited in the sample in the form of phonons. Because the Raman tensor also decomposes into the basis of irreducible representations of the crystal space group, phonons are also classified by their irreducible representation. Raman-active phonon modes can be classified according to the irreducible representations of the crystal space group and its orthogonality.

The laser beam from the argon ion laser is filtered to obtain a monochromatic beam which is directed by a system of mirrors to a focusing/collecting lens and focused onto the sample. The back scattered light from sample is then directed to the spectrometer through filters and gratings. The holographic notch filters allow collection of Raman scattered light closer to the Rayleigh line with less ripple. The holographic transmission gratings enable to acquire entire Raman spectrum simultaneously. The signal as a function of position is read by the system detector which is a multichannel CCD array in which the different positions (wavelengths) are read simultaneously. The wavelengthintensity information is then read to a computer and converted in Andor software to frequency shift versus intensity plots. To resolve a Raman peak of a certain width, the resolution of the spectrometer (final slit width) should be smaller than the peak width. The slit width determines the extent to which the image may shift along the face of the CCD array, and hence the frequency resolution. The dark current of CCD array is a function of the detector temperature which requires it to be cooled. In the present setup CCD is cooled by the use of Joule Thomson effect. When the apparatus is properly aligned, the intensity of Raman spectrum is a function of factors such as the applied laser 
power, the sample properties (how absorptive or reflective the sample is, and the intrinsic strength of the Raman modes), and the width of the slits.

\subsection{Analysis of Powder X-ray Diffraction Data}

\subsubsection{Rietveld method}

It is important to identify strongly overlapping reflections of the powder diffraction patterns to obtain crystal structure information. Rietveld refinement is a technique used to characterize crystalline materials. The height, width and position of the peaks can be used to determine many aspects of the materials structure. The Rietveld method uses a non linear least squares approach to fit or refine a model of crystal structure to the powder diffraction data. This analysis requires determining the structural parameters (unit cell dimensions, atom positions and displacement or thermal parameters) for all crystalline phases present, and a variety of instrumental and sample parameters (scale factors, peak broadening, background and preferred orientation). The analysis can be achieved with approximate starting values for all parameters that will be fitted. Then a computer program is used to optimize (refine) a subset of minimal number of parameters that must be fit. Finally, additional parameters are selected to be refined, until all parameters in the model which the data support are refined.

\subsubsection{Le Bail method}

Le Bail method can be used to get good experimental parameters for background, unit cell and profile before fitting structure. This method estimate best possible fit when profiles are irregular. Also an additional phase can be fitted with Le Bail method where 
structure is not known or where texture is a problem. The Le Bail method caused a marked and radical increase in the ability to solve structures from powder diffraction data. It includes the ability for whole profile unit cell refinement involving overlapping reflections, extraction of structure factors $(|\mathrm{F}| \mathrm{hkls})$ for crystal system and space group determination.

\subsubsection{Data processing and analysis tools used in the study}

The 2D angle dispersive diffraction patterns obtained were integrated using Fit2D [108]. Fit2D is a one and two dimensional data analysis program which can also be used for calibration and correction of detector distortions. GSAS (General Structure Analysis System) is a comprehensive system for the refinement of structural models to both X-ray and neutron diffraction data. The unit cell volumes were determined using Rietveld analysis or Le Bail profile fitting method implemented in the GSAS-EXPGUI software package [109]. The GSAS package can be used with both single-crystal and powder diffraction data. EXPGUI is a graphical user interface (GUI) editor for GSAS experiment (.EXP) files and shell which allows all the other GSAS programs to be executed with a GUI. In addition to structure determination, GSAS is also used for lattice-constant determination even for materials with unknown structures, simulation of powder diffraction data, and for texture analysis $[110,111]$.

Plot85 is used to analyze energy-dispersive diffraction data created by the SAM85 or MCA data collection programs. In Plot85 it is possible to determine the channel number, energy, or d-space for any diffraction peak, either by pointing to the peak, or to 
fit it using GPLS (General Program for Least Squares fitting). If the diffraction data include that of $\mathrm{NaCl}$, the pressure and differential stress can also be calculated [112].

The experimental P-V data were fitted with third order Birch-Murnaghan equation of state using EosFit program [43, 113]. Except the Murnaghan, all other EoSs can be only written with pressure as a function of volume and not vice-versa. Therefore the EosFit program performs least squares fit of $\mathrm{P}-\mathrm{V}$ data with pressure as the dependent variable. Uncertainties in volumes are converted into that of pressure by the effective variance method. The value of $\mathrm{V}_{0}$ is dependent upon the calibration of the technique used to measure the volumes because the refinement of the diffraction pattern is influenced by the alignment of the monochromator, the resulting X-ray wavelength and the detector distance. Volumes measured at high pressures may be on a different scale from some high-accuracy value of $\mathrm{V}_{0}$ determined by another technique. Therefore, the fixing of $\mathrm{V}_{0}$ to an inappropriate value can lead to incorrect estimates of the other EoS parameters. For isothermal data sets, the first stage of refinement should be the refinement of $\mathrm{V}_{0}$ and $\mathrm{K}_{0}$ alone in a second-order EoS, with $\mathrm{K}^{\prime}$ is set to its implied value.

Back ground subtraction and peak fitting were also carried out with Crystal Sleuth and CMPR software packages [114]. Visualization and manipulation of crystal structures were made possible using Powder Cell program [115]. The Raman spectra were fitted with Gaussian profile function in Fityk [116] to obtain Raman shift at different pressures. The correlations of properties of hydrides were studied with statistical package for social sciences (SPSS) program [117]. 


\section{CHAPTER 4. STRUCTURAL STABILITY OF $\mathrm{Mg}_{2} \mathrm{FeH}_{6}$ UNDER HIGH- PRESSURES AND TEMPERATURES}

\subsection{Introduction}

Dimagnesium iron hydride $\left(\mathrm{Mg}_{2} \mathrm{FeH}_{6}\right)$ is widely studied because of its possible application for hydrogen and thermo-chemical heat storage $[118,119] . \mathrm{Mg}_{2} \mathrm{FeH}_{6}$ has the highest volumetric density of hydrogen $\left(\sim 150 \mathrm{kgH}_{2} \mathrm{~m}^{-3}\right)$ among all the hydrides investigated so far and has a gravimetric hydrogen density of $5.4 \mathrm{wt} \% \mathrm{H}_{2}$. Moreover, $\mathrm{Mg}_{2} \mathrm{FeH}_{6}$ is more economical for many applications compared to other hydrides because of the availability and the low cost of component metals $\mathrm{Mg}$ and Fe. The hydride has a $\mathrm{K}_{2} \mathrm{PtCl}_{6}$ type cubic structure with a space group of $\mathrm{Fm}^{-} 3 \mathrm{~m}$, composed of tetrahedral $\mathrm{Mg}^{2+}$ ions and octahedral $\left(\mathrm{FeH}_{6}\right)^{4-}$ complexes. Various research groups have carried out investigations on the synthesis of $\mathrm{Mg}_{2} \mathrm{FeH}_{6}$ [120-130]. Herrich et al.[121], studied the influence of milling conditions and temperature on the hydride formation and obtained a $90 \%$ yield of $\mathrm{Mg}_{2} \mathrm{FeH}_{6}$. Among the compositions investigated a ratio 2: 0.57 of $\mathrm{MgH}_{2}$ to Fe exhibited good results. Decomposition behavior of $\mathrm{Mg}_{2} \mathrm{FeH}_{6}$ was investigated based on synthesis conditions and unreacted $\mathrm{Fe}$ content. The observed decomposition temperatures were $428{ }^{\circ} \mathrm{C}$ and $355^{\circ} \mathrm{C}$ [124]. Desorption mixture of $\mathrm{Mg}_{2} \mathrm{FeH}_{6}$ reversibly absorbs hydrogen to form the hydride at $550{ }^{\circ} \mathrm{C}$ and 60 bar $\mathrm{H}_{2}$ pressure. The presence of $\mathrm{Fe} / \mathrm{MgH}_{2}$ enhances the $\mathrm{H}_{2}$ desorption, however the temperature does not enhance the synthesis rate. Hout et al. [122], reported better reversibility in $\mathrm{Mg}_{2} \mathrm{FeH}_{6}$ synthesized by ball milling of $2 \mathrm{MgH}_{2}+\mathrm{Fe}$ than sintering $2 \mathrm{Mg}+\mathrm{Fe}$ under hydrogen. The enthalpy of 
reaction for $2 \mathrm{MgH}_{2}+\mathrm{FeH}+0.5 \mathrm{H}_{2} \rightarrow \mathrm{Mg}_{2} \mathrm{FeH}_{6}$ is reported to be $-121.402 \mathrm{~kJ} / \mathrm{mol} \mathrm{H}$ and the dissociation enthalpy of $\mathrm{Mg}_{2} \mathrm{FeH}_{6}$ is $98 \mathrm{~kJ} / \mathrm{mol} \mathrm{H}_{2}$ [120].

Studies on $\mathrm{MgH}_{2}$ and $\mathrm{Mg}_{2} \mathrm{FeH}_{6}$ indicated that $\mathrm{Mg}_{2} \mathrm{FeH}_{6}$ reduces the formation heat of the mixture, which suggests that $\mathrm{Mg}_{2} \mathrm{FeH}_{6}$ acts as a catalyst. As a result the milled powder containing $\mathrm{MgH}_{2}$ and $\mathrm{Mg}_{2} \mathrm{FeH}_{6}$ desorbs hydrogen within the small temperature range and exhibits rapid desorption rate $[127,130]$. A reduction in the decomposition temperature of about $100{ }^{\circ} \mathrm{C}$ has been observed for the $\mathrm{Li}$ modified $\mathrm{Mg}_{2} \mathrm{FeH}_{6}$ and an enhancement in the sorption kinetics for the Ti- doped $\mathrm{Mg}_{2} \mathrm{FeH}_{6}$ [131]. Halilov et al. [132], and Orgaz et al. [133], studied electronic structure and chemical bonding in $\mathrm{Mg}_{2} \mathrm{FeH}_{6}$ by first-principal calculations. Calculated energy gap is $\sim 1.8 \mathrm{eV}$ suggesting that $\mathrm{Mg}_{2} \mathrm{FeH}_{6}$ is poor conductor that is consistent with experimental data [133]. However, the calculation shows that the values of the energy gap in $\mathrm{D}_{2} \mathrm{MH}_{6}(\mathrm{D}=\mathrm{Mg}, \mathrm{Ca}, \mathrm{Sr} ; \mathrm{M}=$ $\mathrm{Fe}, \mathrm{Ru}, \mathrm{Os}$ ) series is a function of the unit cell volume. Further studies are required to a better understanding of this feature.

Instead of much experimental work, the high pressure study on $\mathrm{Mg}_{2} \mathrm{FeH}_{6}$ is not yet reported. The transition metal hydride having similar electronic structure, $\mathrm{Mg}_{2} \mathrm{NiH}_{4}$, found to be exhibiting high pressure/temperature phase transitions [134]. Therefore it is worthwhile to study the high pressure- temperature behavior of $\mathrm{Mg}_{2} \mathrm{FeH}_{6}$. High pressure and high temperature X-ray diffraction experiments have been carried out to find the isothermal bulk modulus and thermal expansion coefficient of $\mathrm{Mg}_{2} \mathrm{FeH}_{6}$. 


\subsection{Synthesis and Characterization of $\mathrm{Mg}_{2} \mathrm{FeH}_{6}$}

\subsubsection{Synthesis}

$\mathrm{Mg}_{2} \mathrm{FeH}_{6}$ powder sample was synthesized by mechano- chemical method using a Retsch (PM 100) ball mill. A stoichiometric mixture of $\mathrm{MgH}_{2}$ and $\mathrm{Fe}$ in the ratio 2:1 was ball milled at a speed of $400 \mathrm{rpm}$ under $\mathrm{H}_{2}$ atmosphere for $72 \mathrm{hrs}$ and then annealed at $400{ }^{\circ} \mathrm{C}$ under a $\mathrm{H}_{2}$ pressure of 100 bars. The milling resulted in disappearance of the $\mathrm{MgH}_{2}$ peaks followed by the appearance of $\mathrm{Mg}_{2} \mathrm{FeH}_{6}$ phase which broadened on further milling. After heating the $\mathrm{Mg}_{2} \mathrm{FeH}_{6}$ abundant phase a significant decrease in the full width half maximum (FWHM) of the diffraction peaks was observed.

The X-ray diffraction indicates $\sim 85 \%$ yield of $\mathrm{Mg}_{2} \mathrm{FeH}_{6}$. Particle sizes of the as milled and the annealed samples were determined by the Le Bail profile fitting method in the general structure analysis system (GSAS) using the equation; $D=\frac{\mathbf{5 7 3 2 . 4 8 K \boldsymbol { K }}}{\boldsymbol{L}_{X}}$ where, D -particle size, K- Scherer constant, $\lambda$ - wavelength of X-ray $(0.71073 \AA), L_{x^{-}}$ Lorentzian function representing the particle size broadening [135]. Instrumental particle size broadening of the diffraction peaks was estimated by measurements of $\mathrm{LaB}_{6}$ standard sample with average particle size $1 \mu \mathrm{m}$. The particle sizes were also confirmed by electron microscopy experiments.

\subsubsection{Electron microscopy}

The compositional contrast and sample morphology has been investigated by the scanning electron microscopy (SEM) using Jeol JSM-6330F equipped with energy 
dispersive X-ray spectroscopy (EDS), and by the transmission electron microscopy (TEM) using Philips CM200.

\subsubsection{X-ray diffraction}

The high pressure diffraction studies were conducted at Sector 16-IDB, HPCAT, Advanced Photon Source in the Argonne National Laboratory and the X-ray wavelength was $0.368 \AA$. The sample was loaded into the Mao-Bell type DAC with a culet size of $400 \mu \mathrm{m}$. The sample chamber was a steel gasket having hole of $240 \mu \mathrm{m}$ diameter and 50 $\mu \mathrm{m}$ thick. The high temperature $\mathrm{X}$ - ray diffraction studies were carried out on annealed $\mathrm{Mg}_{2} \mathrm{FeH}_{6}$, using Bruker- GADDS/D8 X-ray system with molybdenum MacSci rotating anode $(\lambda \sim 0.71073 \AA)$ and Apex Smart CCD Detector. For high temperature studies, $\mathrm{Mg}_{2} \mathrm{FeH}_{6}$ was loaded in quartz capillary and kept under vacuum overnight before sealing with Ar gas to avoid oxidation of the sample. A homemade heater coupled with temperature controller was used to heat the sample for in situ XRD experiments up to 500 ${ }^{\circ} \mathrm{C}$. Temperature was calibrated from the thermal expansion of $\mathrm{NaCl}$.

\subsubsection{Computation}

The total energy of $\mathrm{Mg}_{2} \mathrm{FeH}_{6}$ was calculated using the density-functional theory (DFT) within the generalized-gradient approximation (GGA) for the exchangecorrelation function as implemented with a plane-wave basis in the Vienna $a b$ initio simulations package (VASP) [44, 136, 137]. Calculations were performed using the projected-augmented wave (PAW) potentials provided with the VASP [138]. The reciprocal space sampling was done with $k$-point grids of $5 \times 5 \times 5$ which were generated 
according to the Monkhost-Pack scheme. Experimentally established crystal-structure data were used as input [139]. The ionic positions and lattice parameters were relaxed with the conjugated gradient method using forces and stresses for all the volumes considered. In all the calculations, self-consistency was achieved with a tolerance in the total-energy of at least $0.1 \mathrm{meV} /$ atom. The equilibrium volume $\mathrm{V}_{0}$ and bulk modulus $\mathrm{K}_{0}$ were obtained by fitting $\mathrm{dE} / \mathrm{dV}-\mathrm{V}$ (where $\mathrm{V}$ is unit cell volume and $\mathrm{E}$ is its total energy) values to the third order Birch-Murnaghan equation of state.

\subsection{Results and Discussions}

\subsubsection{Electron microscopy}

The selective area electron diffraction (SAD) pattern (dark contrast) shows poly crystalline nature of the sample and the TEM micrograph shows a distribution of particle size between 5-50 nm (Figure 4.1) in the annealed sample. Surface morphology and component distribution of the synthesized $\mathrm{Mg}_{2} \mathrm{FeH}_{6}$ samples were analyzed by SEM. The backscattered and secondary images (Figure 4.2) and the EDS spectra show a homogenous distribution of $\mathrm{Fe}$ and $\mathrm{Mg}$ in the sample. Secondary image show sample morphology of highly agglomerated particles attributing to the high surface energy of the nanoparticles. The weight percentage of different elements from EDS analysis of spots marked 1-3 in the secondary image is summarized in Table 4.1 and the EDS spectrum collected at spot 3 is given in Figure 4.3. 


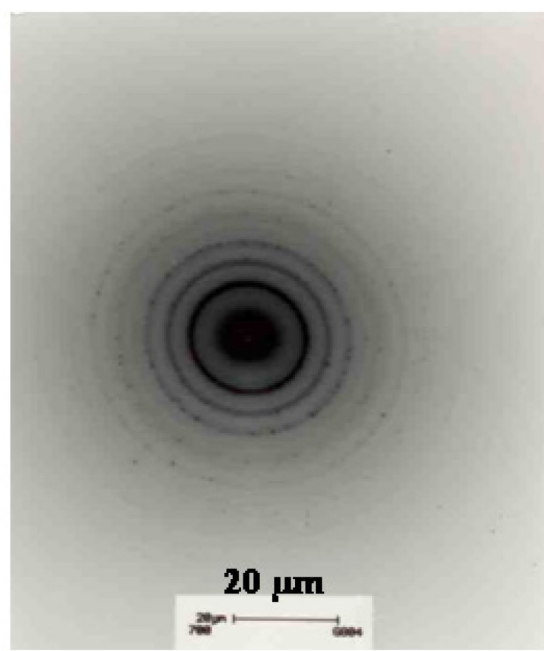

(a)

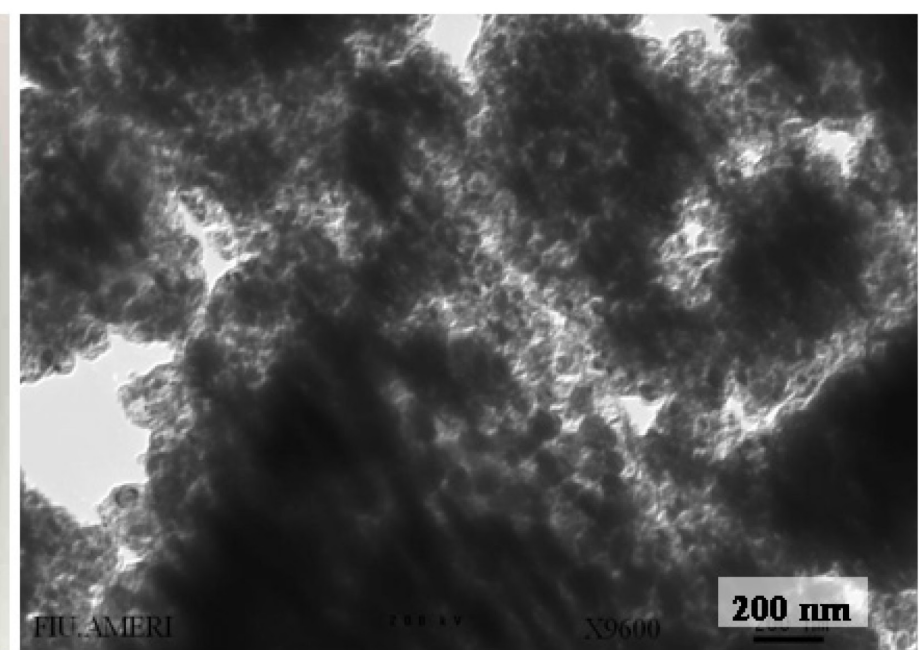

(b)

Figure 4. 1: a) Selective area electron diffraction pattern of annealed $\mathrm{Mg}_{2} \mathrm{FeH}_{6}$. b) TEM image of as synthesized sample.

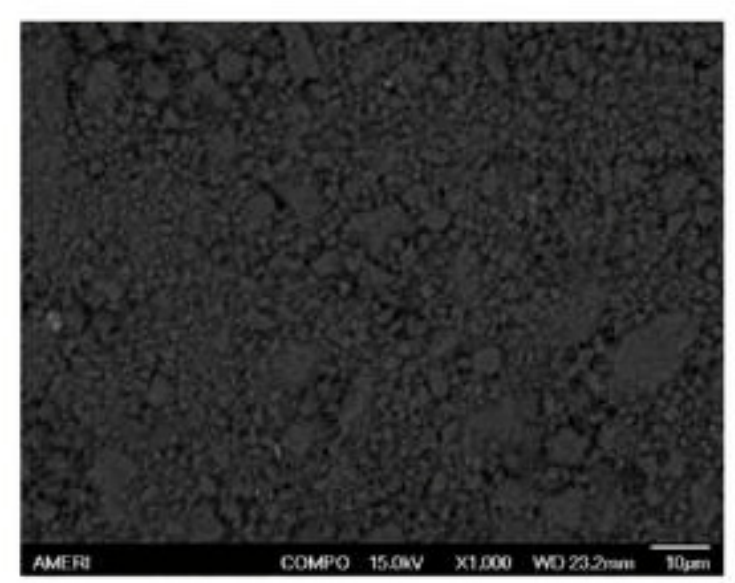

(a)

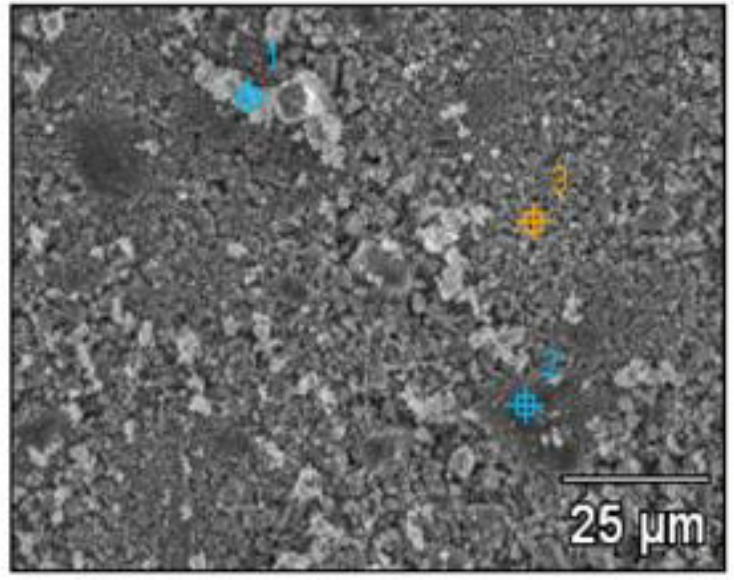

(b)

Figure 4. 2: (a) Backscattered SEM image and; (b) secondary image of annealed $\mathrm{Mg}_{2} \mathrm{FeH}_{6}$. 


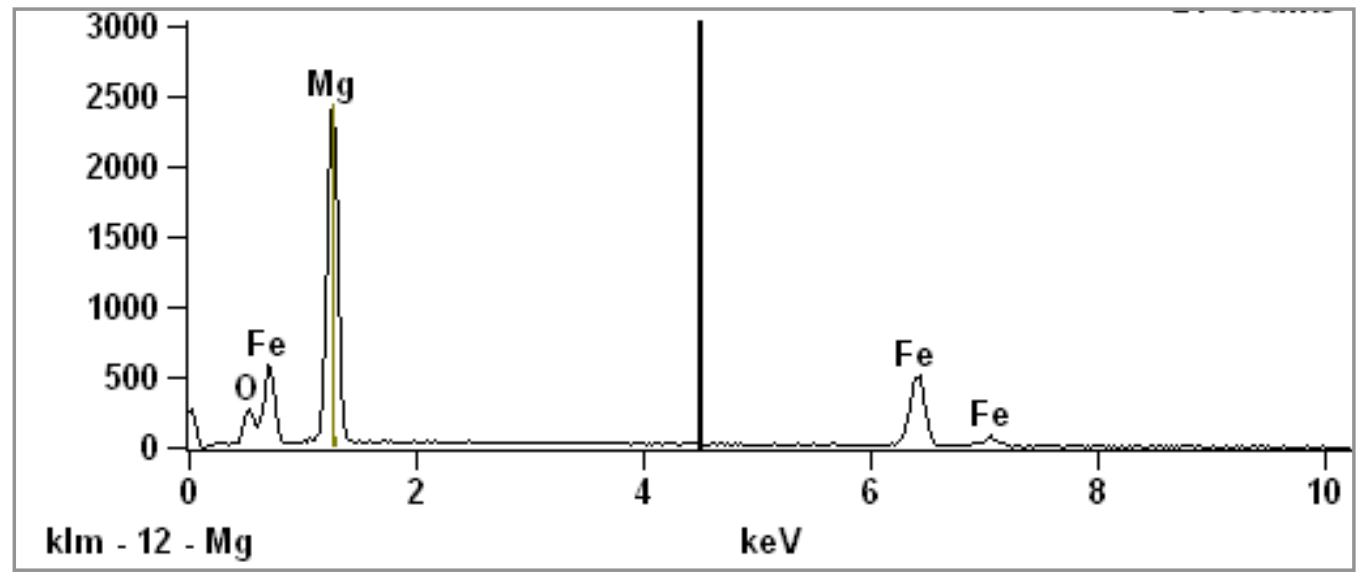

Figure 4. 3: The EDS spectrum collected from spot 3 marked in the secondary image.

Table 4. 1: Weight $\%$ of elemental components $\mathrm{O}, \mathrm{Mg}$ and Fe obtained from EDS analysis at spots marked 1-3 in the secondary image.

\begin{tabular}{llll}
\hline Wt \% & O & Mg & Fe \\
\hline Spot 1 & 8.15 & 43.17 & 48.67 \\
Spot 2 & 9.31 & 41.26 & 49.43 \\
Spot 3 & 7.85 & 40.45 & 51.70 \\
\hline
\end{tabular}

\subsubsection{High pressure micro X-ray diffraction}

High pressure experiments were carried out on both as synthesized and annealed samples to check the influence of ball milling on the properties of $\mathrm{Mg}_{2} \mathrm{FeH}_{6}$. The pressure transmitting medium used in the experiments was silicone oil of viscosity $5 \times 10^{-6} \mathrm{~m}^{2} / \mathrm{s}$ (Sigma- Aldrich). Figure 4.4 shows the synchrotron XRD pattern of $\mathrm{Mg}_{2} \mathrm{FeH}_{6}$ collected at ambient conditions. The Rietveld analysis of the ambient XRD pattern shows it is a cubic- $\mathrm{Fm}^{-} 3 \mathrm{~m}$ phase with a lattice parameter of $\mathrm{a}=6.446(3) \AA$ and a unit cell volume of $\mathrm{V}=267.846(2) \AA^{3}$, which is in good agreement with the earlier reports [122]. The 
atomic positions are; $\mathrm{Mg}-8 \mathrm{c}(0.25,0.25,0.25), \mathrm{Fe}-4 \mathrm{a}(0,0,0)$ and $\mathrm{H}-24 \mathrm{e}(0.2456,0,0)$. A unit cell contains 4 formula units which give a unit cell formula weight of 442.02 $\mathrm{g} / \mathrm{mol}$ and an X-ray density of $2.74 \mathrm{~g} / \mathrm{cm}^{3}$ for the $\mathrm{Mg}_{2} \mathrm{FeH}_{6}$. Weight fraction of $\mathrm{Mg}_{2} \mathrm{FeH}_{6}$ was $85.4 \%$ and $\mathrm{Fe}$ was $14.5 \%$. A small fraction of $\mathrm{MgO}$ and $\mathrm{Mg}$ were also present in the sample. Particle size of as milled and the annealed samples are found to be $13.6 \mathrm{~nm}$ and $53.84 \mathrm{~nm}$, respectively. Both compression and decompression data of $\mathrm{Mg}_{2} \mathrm{FeH}_{6}$ were collected in various steps between $0.2-36.5 \mathrm{GPa}$.

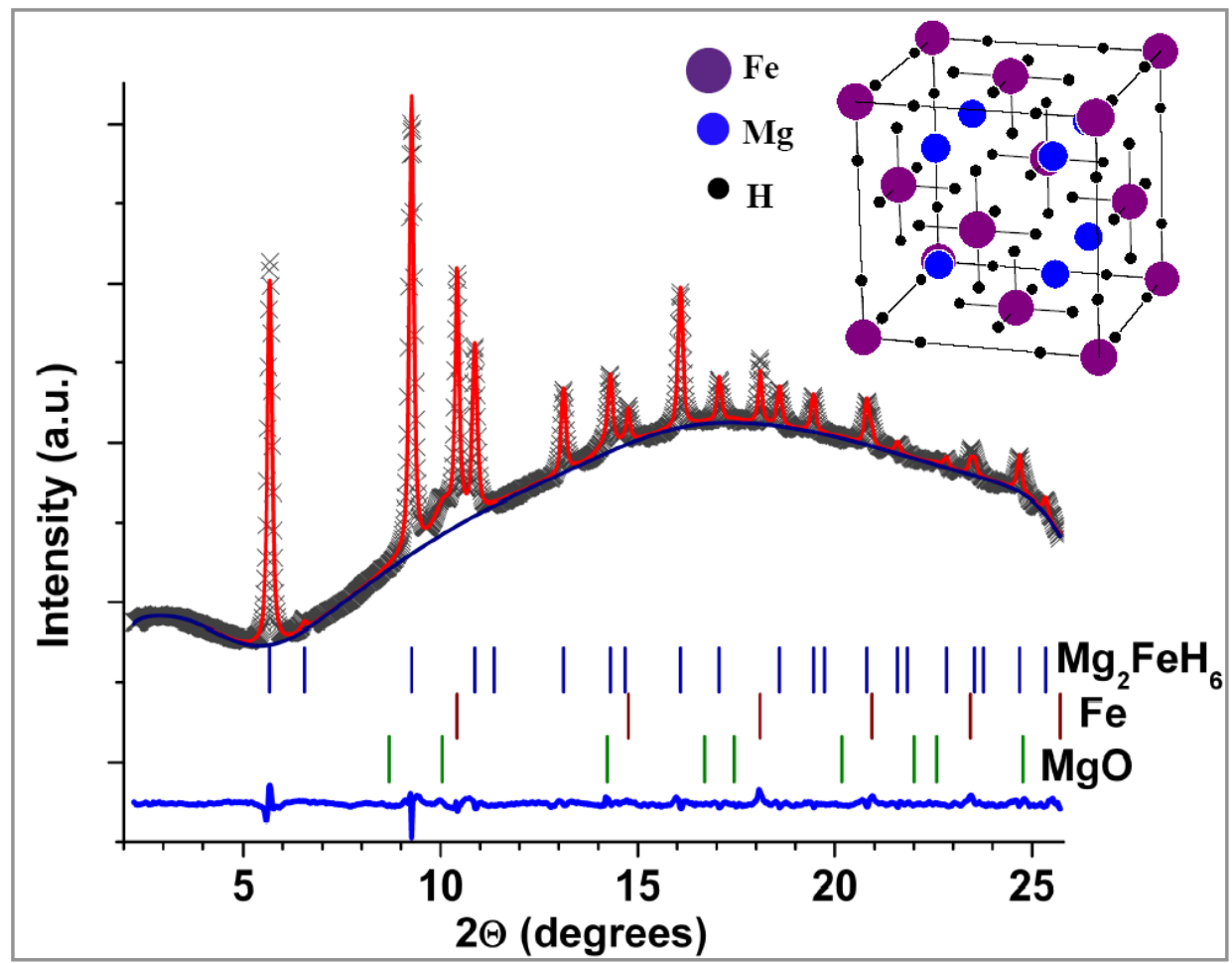

Figure 4. 4: Synchrotron XRD pattern collected at ambient conditions with the results of the Rietveld analysis. Inset is the simulated crystal structure of $\mathrm{Mg}_{2} \mathrm{FeH}_{6}$.

Unit cell volumes at different pressure are determined by peak fitting with the CMPR software package [114]. Figure 4.5 shows the X-ray diffraction patterns collected 
for the annealed samples at various pressures. No structural phase transition was observed up to the highest pressure reached in the experiment. The change in the XRD peak positions corresponding to the $\mathrm{Fe}$, above $10.6 \mathrm{GPa}$, is attributed to the bcc to hcp phase transition $[140,141]$. The peaks corresponds to hcp iron is overlapping with sample peaks, yet clearly visible in the XRD patterns collected above $25 \mathrm{GPa}$.
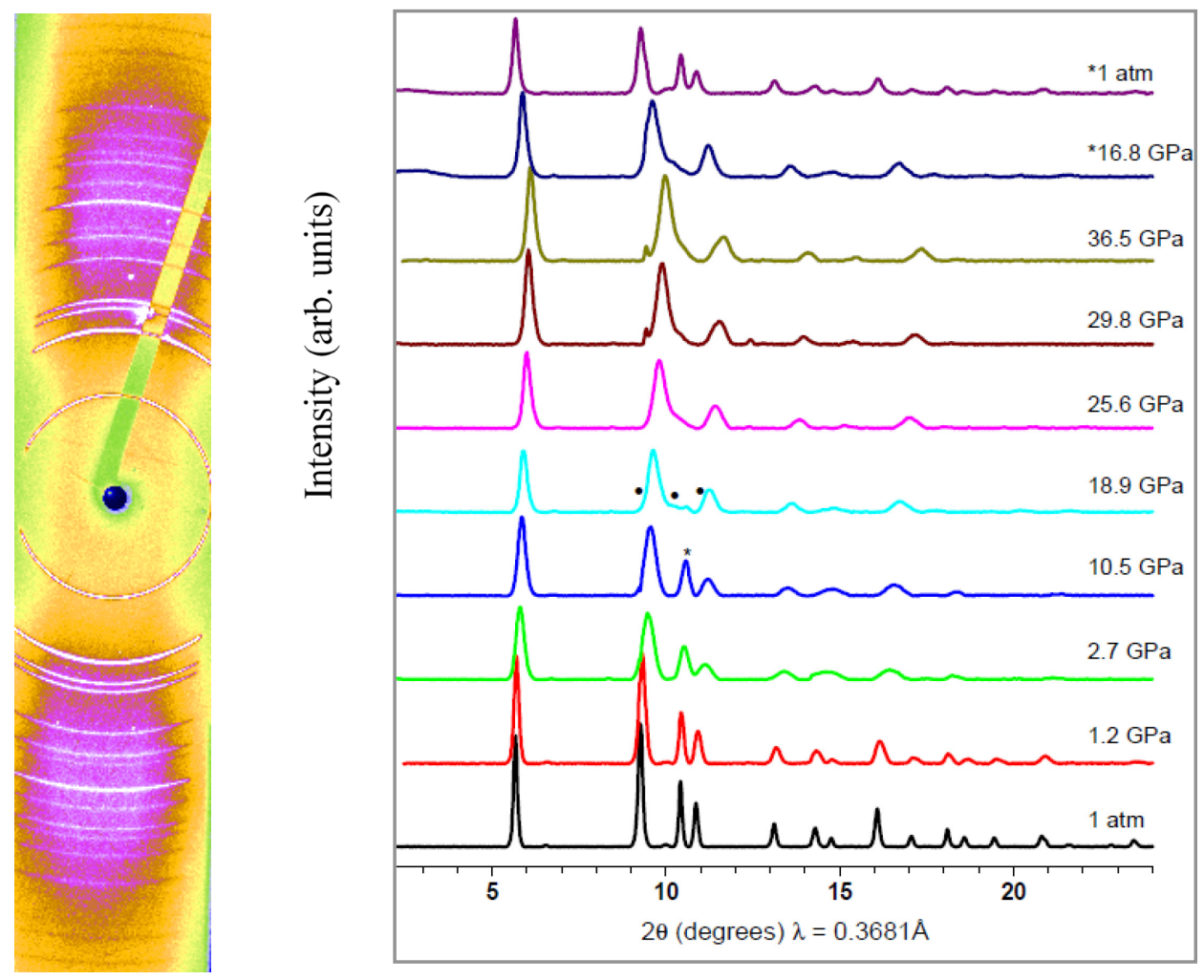

(a)

(b)

Figure 4. 5: (a) Debye diffraction rings collected from sample loaded in DAC, at ambient conditions. (b) X-ray diffraction patterns of $\mathrm{Mg}_{2} \mathrm{FeH}_{6}$ for some selected pressures during compression and decompression (pressure indicated by *). Peaks correspond to hcp phase of Fe are marked by closed circles in the patterns at $18.9 \mathrm{GPa}$. 
The bulk modulus data was determined by fitting the P-V data, Figure 4.6, with the third order Birch-Murnaghan equation of state. Bulk modulus of $\mathrm{Mg}_{2} \mathrm{FeH}_{6}$ calculated using the VASP was 76.3(0) GPa, while the experimental values are in the range 75-170 GPa for the samples measured at different conditions. The value of bulk modulus obtained from X-ray diffraction analysis of the sample with pressure medium was comparable with the calculated value. Table 4.2 shows a comparison of the isothermal $\mathrm{K}_{0}$ data obtained from high pressure studies on various samples of $\mathrm{Mg}_{2} \mathrm{FeH}_{6}$ with $\mathrm{K}_{0}^{\prime}$ fixed to 4 .

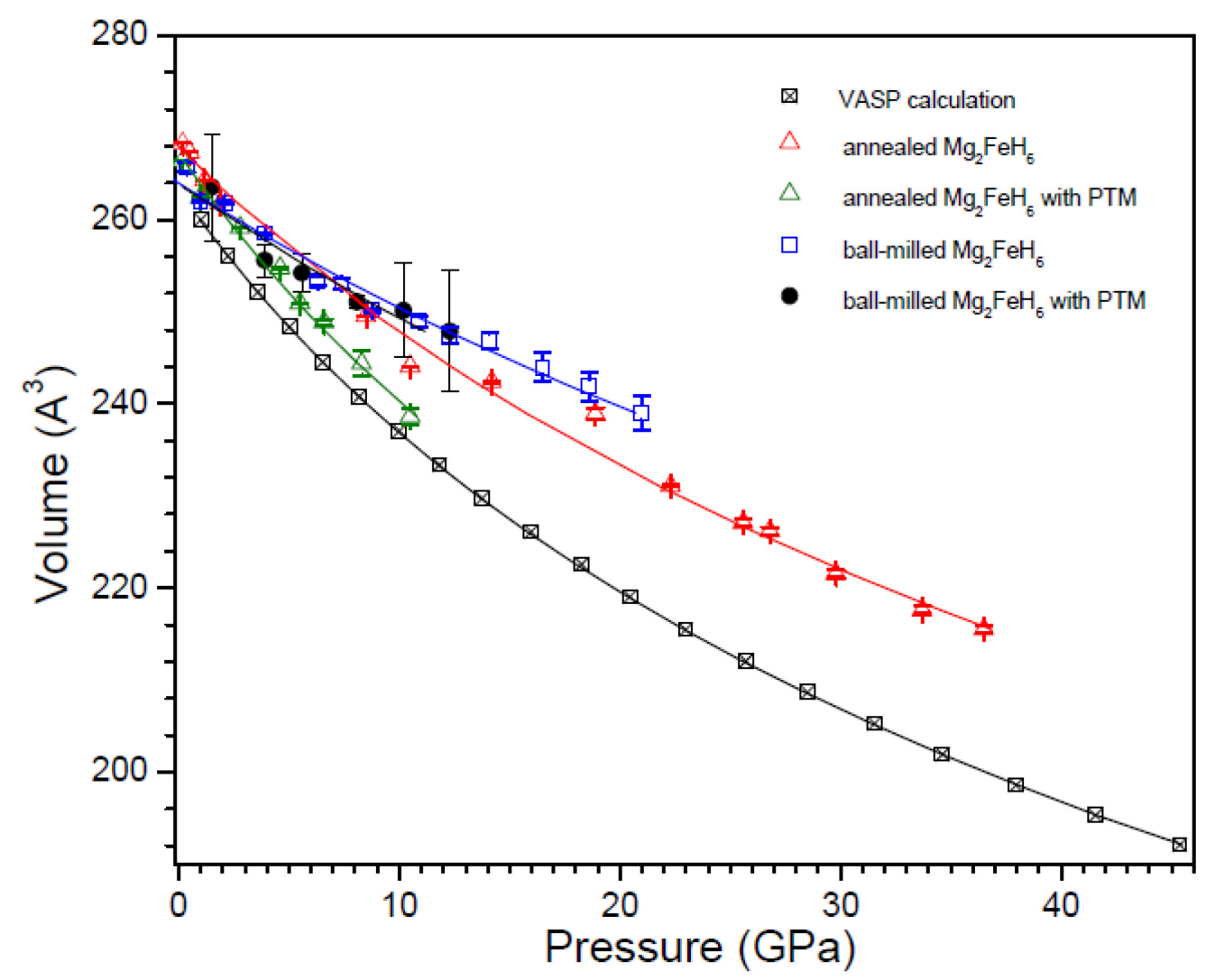

Figure 4. 6: A comparison of calculated and experimental variation of unit cell volume of $\mathrm{Mg}_{2} \mathrm{FeH}_{6}(\mathrm{MFH})$ with pressure fitted with the third order Birch-Murnaghan EoS under different conditions. PTM abbreviates pressure transmitting medium. 
Table 4. 2: Data obtained from fitting P-V data with Birch-Murnaghan EoS for various $\mathrm{Mg}_{2} \mathrm{FeH}_{6}$ samples with a comparison of pressure ranges. PTM abbreviates pressure transmitting medium.

\begin{tabular}{|c|c|c|c|c|c|}
\hline $\mathrm{Mg}_{2} \mathrm{FeH}_{6}$ & $\begin{array}{l}\text { P-range } \\
\text { (GPa) }\end{array}$ & $V_{0}\left(\AA^{3}\right)$ & $\mathrm{K}_{\mathbf{0}}(\mathrm{GPa})$ & $\begin{array}{l}V_{0}\left(\AA^{3}\right) \\
\text { P- range }(\end{array}$ & $\begin{array}{l}\mathrm{K}_{0}(\mathrm{GPa}) \\
10 \mathrm{Gpa}\end{array}$ \\
\hline VASP & $0-45$ & $263.51(0)$ & $76.31(0)$ & $263.24(0)$ & $77.07(1)$ \\
\hline annealed & $0-37$ & $268.2(1)$ & $108.75(3)$ & 267.61(5) & $95.26(5)$ \\
\hline ball milled & $0-21$ & $264.09(1)$ & $170.36(8)$ & $265.28(1)$ & $139.05(10)$ \\
\hline annealed+ PTM & $0-10.5$ & $267.62(1)$ & $75.35(4)$ & $267.62(1)$ & $75.35(4)$ \\
\hline ball milled+ PTM & $0-12.3$ & $264.02(2)$ & $157.11(27)$ & $264.81(2)$ & $139.15(31)$ \\
\hline
\end{tabular}

As synthesized samples show high bulk modulus, with and without pressure medium. This can be attributed to the largely nano- morphology of the $\mathrm{Mg}_{2} \mathrm{FeH}_{6}$ sample synthesized by ball milling. A close analogue of dimagnesium iron hydride, $\mathrm{Mg}_{2} \mathrm{NiH}_{4}$, has the bulk modulus of $43 \mathrm{GPa}$ [134]. Barsan et al. [142], studied ruthenium hydrides $\mathrm{M}_{2} \mathrm{RuH}_{6}(\mathrm{M}=\mathrm{Ca}, \mathrm{Sr}$ and $\mathrm{Eu})$ with a $\mathrm{K}_{2} \mathrm{PtH}_{6^{-}}$type crystal structure by Raman spectroscopy up to $5 \mathrm{GPa}$. They reported bulk moduli of $18.8 \mathrm{GPa}$ for $\mathrm{Ca}_{2} \mathrm{RuH}_{6}, 26.73$ GPa for $\mathrm{Sr}_{2} \mathrm{RuH}_{6}$ and $20.38 \mathrm{GPa}$ for $\mathrm{Eu}_{2} \mathrm{RuH}_{6}$. Those values were determined in the assumption of linear correlation between cubic unit lattice parameter and vibrational frequencies. However, these values might be much underestimated taking into account the current work and the calculated bulk moduli of $\mathrm{Ca}_{2} \mathrm{RuH}_{6}$, which are $67.6 \mathrm{GPa}$ (LDA) and 58.5 GPa (GGA) obtained by DFT [143]. The bulk modulus values obtained by fitting data up to $10.5 \mathrm{GPa}$ are compared with that of the data at different rages of pressure in the Table 4.2. Results show that there is no effect of silicone oil in the bulkmodulus values obtained for the ball milled sample below $10.5 \mathrm{GPa}$. This confirms 
the assumption that the high bulk modulus of the ball milled materials is caused by stress or particle size reduction induced by milling.

\subsubsection{High temperature X-ray diffraction}

Figure 4.7 shows the high temperature in situ X-ray diffraction patterns of $\mathrm{Mg}_{2} \mathrm{FeH}_{6}$ collected at various temperatures up to $450{ }^{\circ} \mathrm{C}$. No temperature induced structural phase transition was observed in $\mathrm{Mg}_{2} \mathrm{FeH}_{6}$ before its decomposition around 425 ${ }^{\circ} \mathrm{C}$. X-ray diffraction pattern collected at $450{ }^{\circ} \mathrm{C}$ shows the presence of $\mathrm{Fe}, \mathrm{Mg}$ and a small amount of $\mathrm{MgO}$.

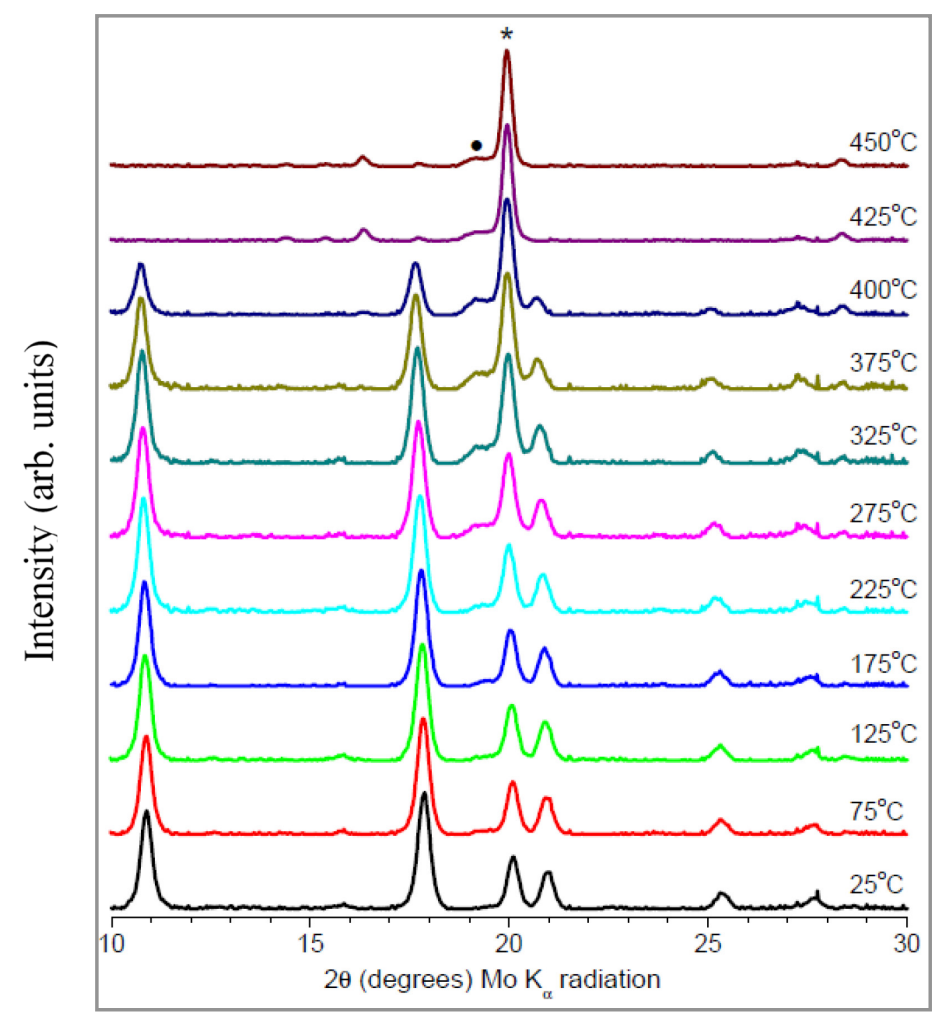

Figure 4. 7: High temperature $\mathrm{XRD}$ patterns of the $\mathrm{Mg}_{2} \mathrm{FeH}_{6}$ collected at various temperatures. In the pattern taken at $450{ }^{\circ} \mathrm{C}$, peak marked by asterisks is due to $\mathrm{Fe}$ and that marked by closed circle is $\mathrm{MgO}$, and others are of $\mathrm{Mg}$. 
The volumetric thermal expansion is calculated from the unit cell volume and temperature using the relation, $\alpha_{v}=\frac{1}{V_{o}} \frac{d V}{d T}$. Second order polynomial fit to the $\Delta \mathrm{V} / \mathrm{V}_{0}$ versus $\mathrm{T}_{-} \mathrm{T}_{0}$ (Figure 4.8) gives thermal expansion as $\alpha_{\mathrm{v}}=5.85(3) 10^{-5}+7.47(7) 10^{-8}\left(\mathrm{~T}-\mathrm{T}_{0}\right) /$ ${ }^{\circ} \mathrm{C}$. This value is 10 times higher than the thermal expansion coefficient reported for the $\mathrm{ZrH}_{2}[144]$.

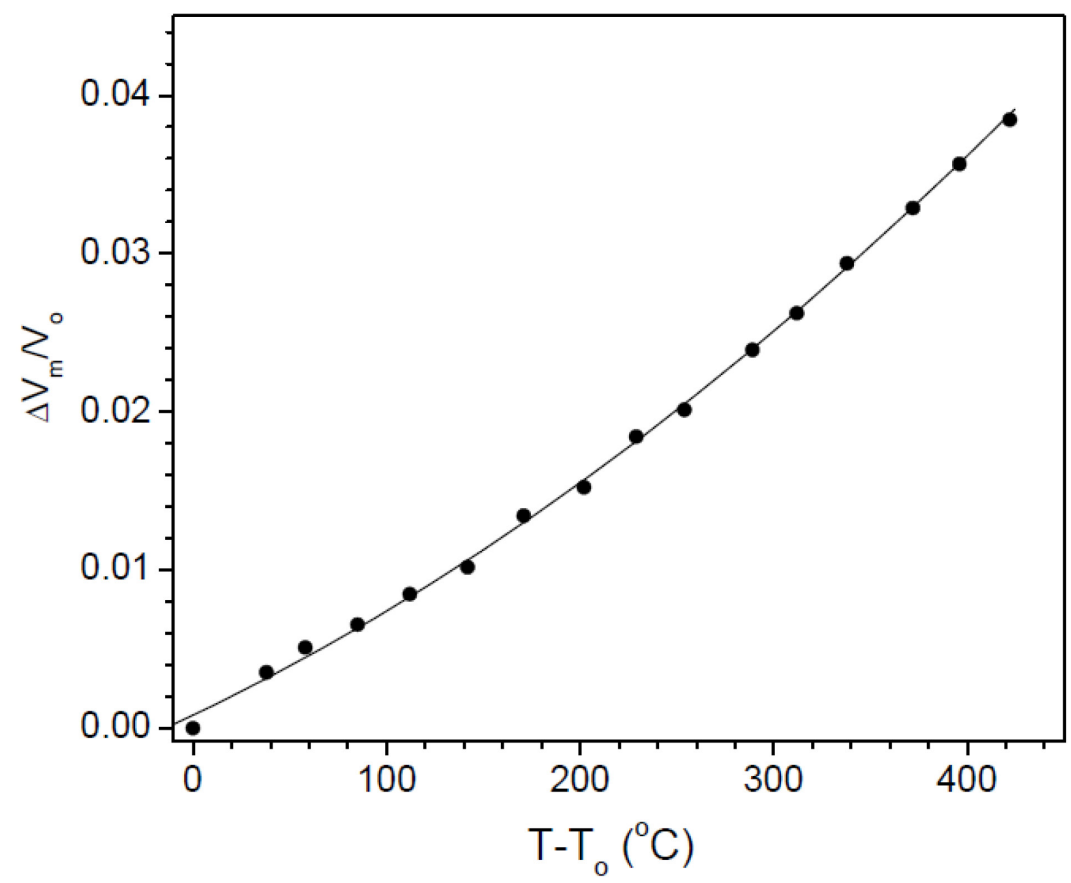

Figure 4. 8: Plot of normalized volume versus temperature for $\mathrm{Mg}_{2} \mathrm{FeH}_{6}$ fitted with a second order polynomial.

\subsubsection{Raman spectroscopy}

Parker et al. [145], has reported Raman spectra of $\mathrm{Mg}_{2} \mathrm{FeH}_{6}$ and the laser power on the sample was $4 \mathrm{~mW}$. The output laser power used in the present study was between 10 and $15 \mathrm{~mW}$ which is the least achievable value in the Raman spectrometer used. The 
results show intense peaks of $\mathrm{Fe}-\mathrm{H}$ bending modes in the range $800-1100 \mathrm{~cm}^{-1}$. Fe-H stretching modes (1600-2000 $\left.\mathrm{cm}^{-1}\right)$ are very weak which can be attributed to laser induced partial decomposition or bonding structure change. The liberational modes are observed at 356 and $585 \mathrm{~cm}^{-1}, \mathrm{Fe}-\mathrm{H}$ bending modes at 787 and $1093 \mathrm{~cm}^{-1}$ and $\mathrm{Fe}-\mathrm{H}$ stretching mode at $1866 \mathrm{~cm}^{-1}$. Figure 4.9 shows Raman spectrum (background subtracted) collected from $\mathrm{Mg}_{2} \mathrm{FeH}_{6}$ sample loaded in a quartz capillary at ambient conditions.

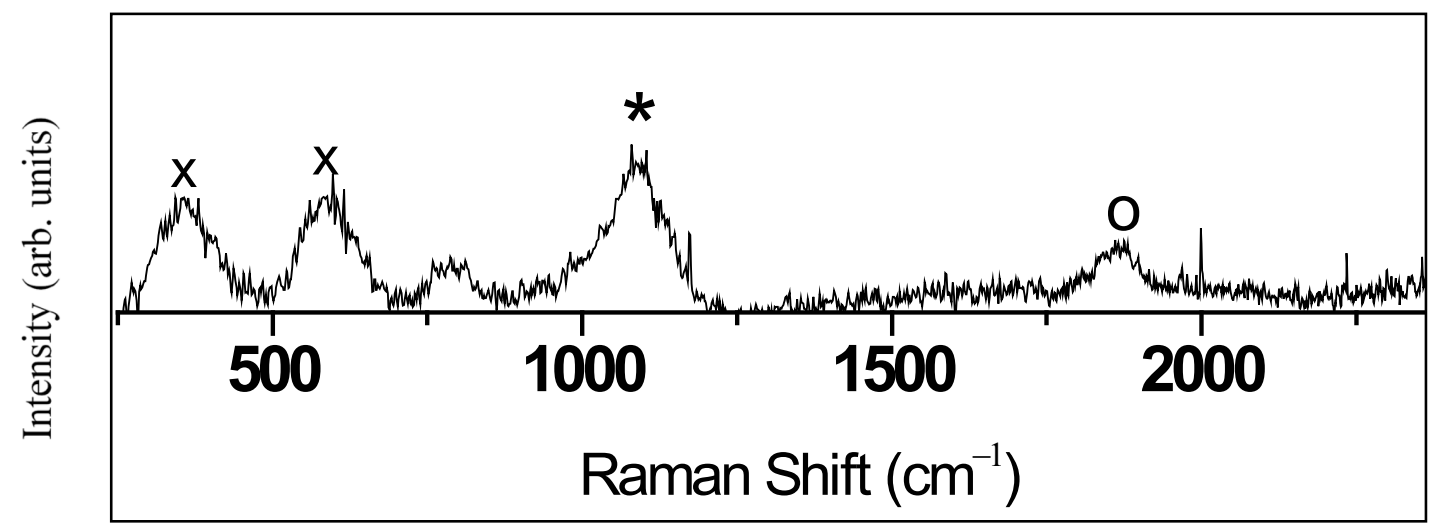

Figure 4. 9: Raman spectrum of $\mathrm{Mg}_{2} \mathrm{FeH}_{6}$ at ambient conditions. Liberational modes (x); Fe-H bending modes (*); Fe-H stretching mode (o). 


\section{CHPATER 5. STRUCTURAL STABILITY OF DIFFERENT \\ POLYMORPHS OF $\mathrm{Mg}\left(\mathrm{BH}_{4}\right)_{2}$}

\subsection{Introduction}

Magnesium borohydride, $\mathrm{Mg}\left(\mathrm{BH}_{4}\right)_{2}$, is a promising lightweight solid state hydrogen storage material with a theoretical hydrogen capacity of $14.8 \mathrm{wt} \%$. The thermodynamical properties of $\mathrm{Mg}\left(\mathrm{BH}_{4}\right)_{2}$ were examined by several researchers and it was found that reaction enthalpy and entropy should give 1 bar equilibrium pressure for hydrogen at room temperature [38, 146, 147]. The low enthalpy of decomposition suggests a possibility of reversible hydrogen storage in this compound [78, 79, 147]. However, it seems as if there were high kinetic barriers involved in the hydrogen desorption leading to high operation temperature $\left(>270{ }^{\circ} \mathrm{C}\right)$, poor kinetics and difficulty to re-hydrogenate the desorption product. The possible decomposition steps for $\mathrm{Mg}\left(\mathrm{BH}_{4}\right)_{2}$ may be; $\mathrm{Mg}\left(\mathrm{BH}_{4}\right)_{2} \rightarrow \mathrm{MgH}_{2}+2 \mathrm{~B}+3 \mathrm{H}_{2} \rightarrow \mathrm{Mg}+2 \mathrm{~B}+4 \mathrm{H}_{2} \rightarrow \mathrm{MgB}_{2}+4 \mathrm{H}_{2}$ [146]. The investigation of Ozolins et al. [148], lead to the conclusion that thermodynamically reversible hydrogen storage is possible in $\mathrm{Mg}\left(\mathrm{BH}_{4}\right)_{2}$ but it is kinetically hindered. Moreover, calcium analogue of $\mathrm{Mg}\left(\mathrm{BH}_{4}\right)_{2}$ has been demonstrated recently to show reversible hydrogen storage properties [149-151]. To improve the hydrogen absorption/ desorption properties of $\mathrm{Mg}\left(\mathrm{BH}_{4}\right)_{2}$, additives like Ti are explored [146]. To verify such phenomenon in $\operatorname{Mg}\left(\mathrm{BH}_{4}\right)_{2}$ further research is inevitable by theoretical calculations of thermodynamic properties which in turn requires a proper structural data in a wide range of temperatures and pressures. Moreover, destabilization of metal borohydrides was 
suggested to be achieved by application of high pressure via formation of short $\mathrm{HI}$ distances and deformation of tetrahedral $\mathrm{BH}_{4}$ anions $[79,152]$.

There are many reports on synthesis and crystal structure of $\operatorname{Mg}\left(\mathrm{BH}_{4}\right)_{2}[12,38$, 78, 79, 95, 153-157]. Her et al. [95], reported that crystal structure of $\operatorname{Mg}\left(\mathrm{BH}_{4}\right)_{2}$ is hexagonal- $\mathrm{P} 6_{1}$ below $180{ }^{\circ} \mathrm{C}$ and above this temperature it transforms to an orthorhombic- Fddd phase which is stable up to the decomposition temperature of 340 ${ }^{\circ} \mathrm{C}$. The $P 6_{1}$ structure of $\mathrm{Mg}\left(\mathrm{BH}_{4}\right)_{2}$ with 30 formula units (330 atoms) in a unit cell contains five symmetry independent $\mathrm{Mg}^{2+}$ cations and ten $\mathrm{BH}_{4}{ }^{-}$anions. Hydrogen coordination for $\mathrm{Mg}^{2+}$ is 8 and locally the $\mathrm{Mg}^{2+}$ network resembles an amorphous state [153]. The density of low temperature phase of $\mathrm{Mg}\left(\mathrm{BH}_{4}\right)_{2}$ is found to be $0.78 \mathrm{~g} / \mathrm{cm}^{3}$ whereas that of high temperature phase is $0.76 \mathrm{~g} / \mathrm{cm}^{3}$. The structure of high temperature phase was found to be coherent in $b-c$ plane but not in $a$-direction. The bonding in $\operatorname{Mg}\left(\mathrm{BH}_{4}\right)_{2}$ is partially covalent similar to that of $\mathrm{MgH}_{2}$ but it has a higher bond strength than $\mathrm{MgH}_{2}$ [12]. The B-H interaction in $\mathrm{BH}_{4}^{-}$tetrahedra is iono-covalent and that between $\mathrm{Mg}$ and $\mathrm{H}$ is ionic [78]. The hydrogen and $\mathrm{BH}_{4}{ }^{-}$co-ordination of $\mathrm{Mg}^{2+}$ ion is strongly related to its ionic radius/ ionic character which may be a cause of the complex bonding nature of $\mathrm{Mg}^{2+}$ ions. First-principal density functional theory calculations failed to predict ambient-pressure structure of $\operatorname{Mg}\left(\mathrm{BH}_{4}\right)_{2}$ due to its complexity [147, 148].

Because of the observed irreversibility of high temperature phase transition, high pressure behavior of $\mathrm{Mg}\left(\mathrm{BH}_{4}\right)_{2}$ is of special interest. Therefore more studies are required to find a method to accelerate the kinetics of the compound formation. To determine the proper additive to enhance kinetics of $\operatorname{Mg}\left(\mathrm{BH}_{4}\right)_{2}$, the actual structure and structural modifications of this compound should be understood. Many aspects of phase transition 
in $\mathrm{Mg}\left(\mathrm{BH}_{4}\right)_{2}$ is still unknown and to be solved for better understanding of the factors affecting the polymorphism. In order to verify structural predictions and to compare the behavior of high pressure phase to that of high temperature phase for $\operatorname{Mg}\left(\mathrm{BH}_{4}\right)_{2}$, micro X-ray diffraction and Raman spectroscopic studies were carried out up to $22 \mathrm{GPa}$. The results indicate an irreversible pressure induced phase transition in $\operatorname{Mg}\left(\mathrm{BH}_{4}\right)_{2}$.

\subsection{Experimental Details}

\subsubsection{Synthesis}

Powder sample of $\mathrm{Mg}\left(\mathrm{BH}_{4}\right)_{2}$ was synthesized by wet chemical method of reaction between $\mathrm{MgH}_{2}$ and triethylamine borane complex under an argon ambient followed by heating above $100{ }^{\circ} \mathrm{C}$ in two steps [146]. The mixture was then cooled to room temperature, filtered and washed with n-hexane and dried under vacuum. The triethylamine adduct of $\mathrm{Mg}\left(\mathrm{BH}_{4}\right)_{2}$ obtained was heated at various steps up to $160{ }^{\circ} \mathrm{C}$ under vacuum which yielded $98 \%$ pure sample. Using an argon-filled glove-box, the sample were loaded into the Mao-Bell type DAC of a culet size $400 \mu \mathrm{m}$ and the sample chamber was a stainless steel gasket with a hole of size $190 \mu \mathrm{m}$ and thickness $50 \mu \mathrm{m}$.

\subsubsection{High pressure micro X-ray diffraction}

The high pressure angle-dispersive micro XRD studies were conducted at Sector 16-IDB, HPCAT, Advanced Photon Source in Argonne National Laboratory $(\lambda=$ $0.368138 \AA$ ) and at X17C beam line of National Synchrotron Light Source in Brookhaven National Laboratory $(\lambda=0.4066 \AA)$. 


\subsection{Results and Discussion}

5.3.1. X-ray diffraction of as synthesized $\mathrm{Mg}\left(\mathrm{BH}_{4}\right)_{2}$

Figure 5.1 shows the micro XRD pattern of $\mathrm{Mg}\left(\mathrm{BH}_{4}\right)_{2}$ pressurized to $\sim 1 \mathrm{GPa}$ and the corresponding simulated pattern of hexagonal $P 6_{1}$ structure. Result shows that below 23.5 GPa, $\mathrm{Mg}\left(\mathrm{BH}_{4}\right)_{2}$ is a single phase and has a hexagonal $P 6_{1}$ structure with lattice parameters $a=10.047(3) \AA, c=36.34(1) \AA$ and $V=3176(1) \AA^{3}$ at $\sim 0.2$ GPa which agree with the previously reported values [79, 156].

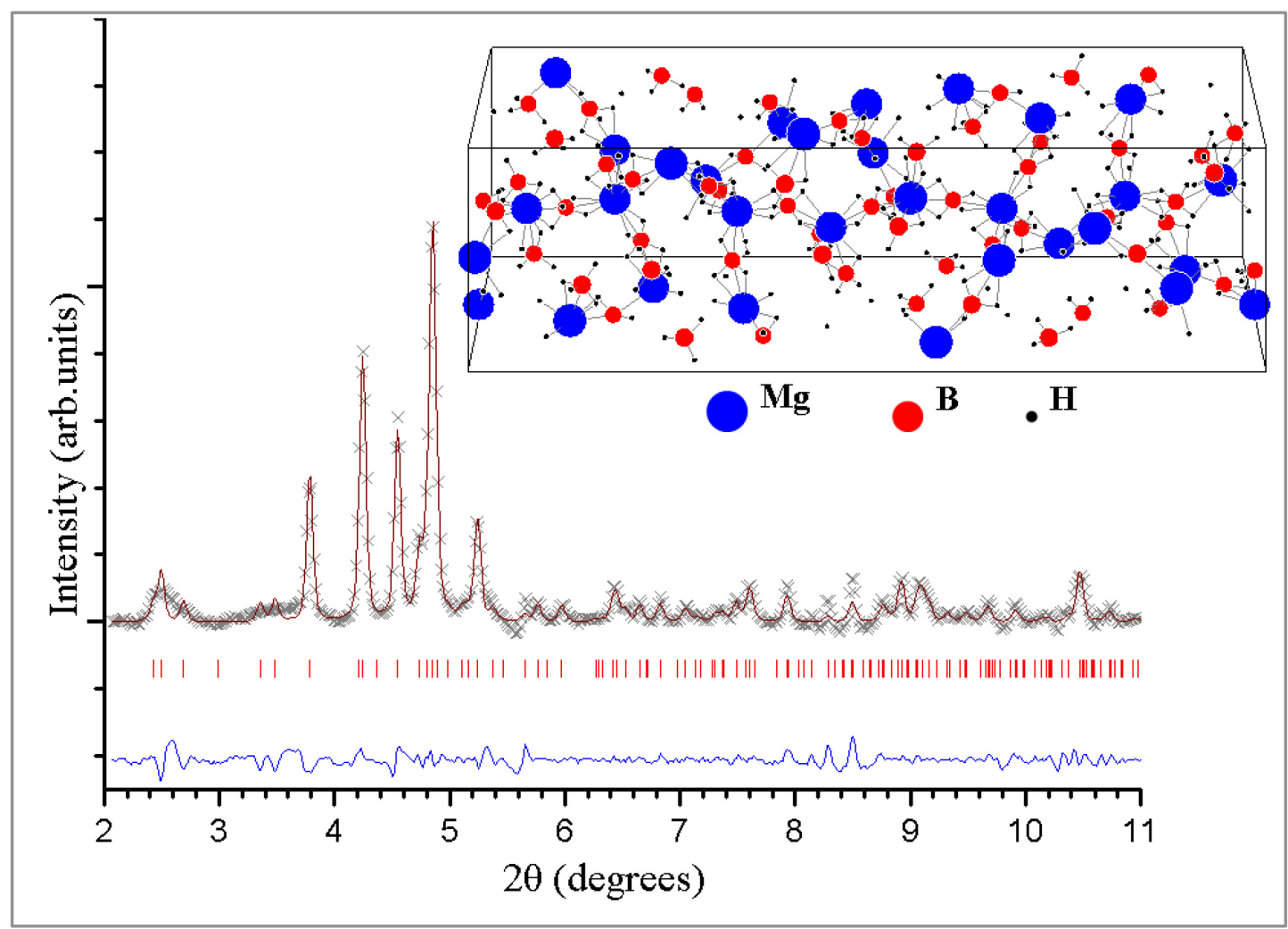

Figure 5. 1: Synchrotron XRD pattern of low pressure phase of $\mathrm{Mg}\left(\mathrm{BH}_{4}\right)_{2}$ at $\sim 1 \mathrm{GPa}$ with the results of Le Bail profile fitting and inset shows the corresponding hexagonal $\mathrm{P}_{1}$ structure. 
Figure 5.2 shows synchrotron XRD patterns recorded during compression and decompression at various pressures up to $\sim 11 \mathrm{GPa}$. The ambient pressure and high pressure phases coexist in the range 1.5-2.4 GPa. At 3.35 GPa the transition is complete. The high pressure phase is stable up to the highest pressure reached in the X-ray diffraction experiments. Pressure-transmitting medium was not used in the synchrotron XRD experiments. The decompression data shows the high pressure phase transition is irreversible.

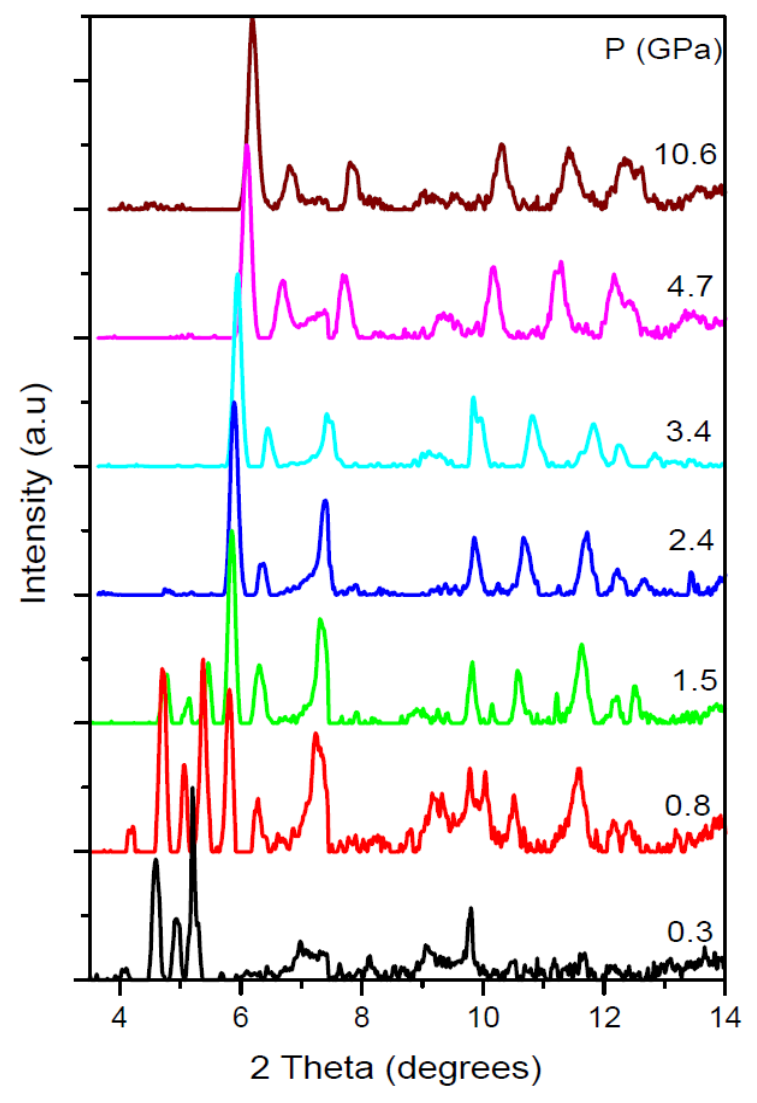

(a)

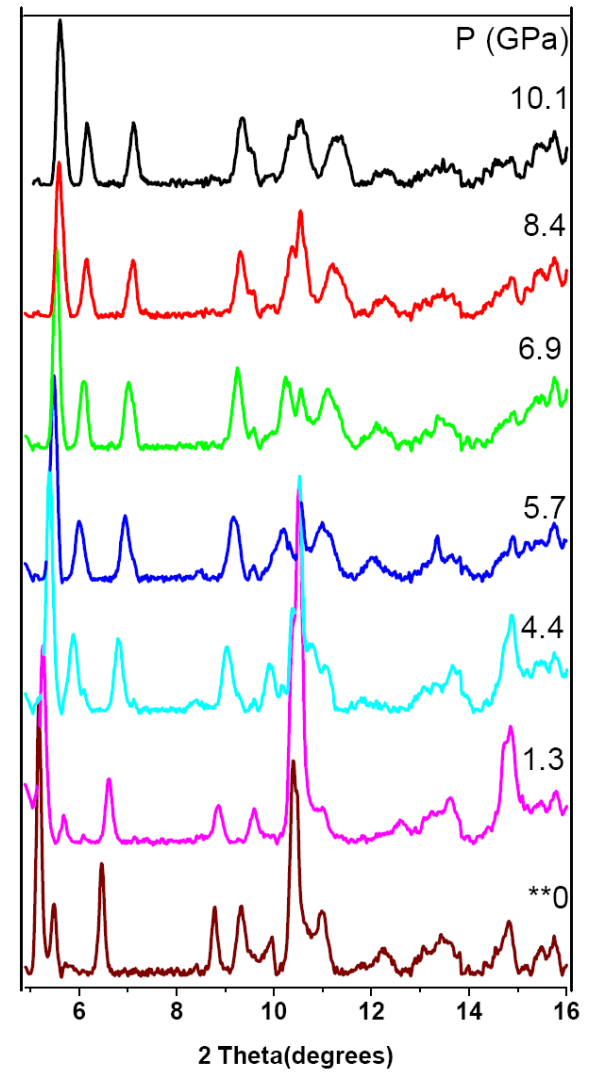

(b)

Figure 5. 2: The synchrotron $\mathrm{XRD}$ pattern obtained for $\mathrm{Mg}\left(\mathrm{BH}_{4}\right)_{2}$; (a) during compression $(\lambda=0.4066 \AA)$ up to $\sim 11 \mathrm{GPa}$, (b) during decompression $\chi=0.368138 \AA$ ) at various pressures. 
The irreversibility of the high pressure transformation even after the release of pressure suggests that the reverse transformation is kinetically hindered or ambient pressure polymorph of $\mathrm{Mg}\left(\mathrm{BH}_{4}\right)_{2}$ is a metastable phase. The later might be indicating that the structure of $\operatorname{Mg}\left(\mathrm{BH}_{4}\right)_{2}$ at ambient conditions can be influenced by the synthesis and processing conditions. The similar situation is observed for aluminum hydride, $\mathrm{AlH}_{3}$, with six different phases which can be prepared via chemical route by varying synthesis conditions [158]. Another aspect should be noted is that the ambient pressure structure of magnesium borohydride is characterized by large degree of disorder which might have kinetics origin, application of high external pressure eliminates this disorder and leads to more ordered and thus more simple structure.

Because of the low intensity of $\operatorname{Mg}\left(\mathrm{BH}_{4}\right)_{2}$ XRD patterns, solving the structure of high pressure phase was difficult. It is observed that the structure of $\mathrm{Mg}\left(\mathrm{BH}_{4}\right)_{2}$ at high pressures is different from the high temperature orthorhombic- Fddd phase [95]. It is also verified that it does not match with the structures predicted by first-principal calculations such as trigonal- $P-3 m 1$, monoclinic- $P 2 / c$ [147], tetragonal- $I-4 m 1$ [148], or orthorhombic- $P m c 2_{1}$ [78]. The possible decomposition products of magnesium borohydride such as $\mathrm{MgB}_{2}, \mathrm{Mg}$ or $\mathrm{B}$ were also absent in the XRD patterns of the high pressure phase.

The XRD patterns of high pressure phase were indexed in hexagonal $\mathrm{P}_{3}$ space group with lattice parameters at $5.5 \mathrm{GPa}: a=8.35(5) \AA, \mathrm{c}=4.68(4) \AA$ and $V=283.47(8)$ $\AA^{3}$. Figure 5.3 shows variation of molar volume with pressure for the $\mathrm{Mg}\left(\mathrm{BH}_{4}\right)_{2}$ up to $11 \mathrm{GPa}$. The P-V data of high pressure phase were fitted with third order BirchMurnaghan equation of state and the bulk modulus was found to be 10.16(6) GPa with $\mathrm{V}_{\mathrm{o}}$ 
$=378.12(7) \AA^{3}$ and $\mathrm{K}_{0}{ }^{\prime}$ is fixed to 4 . The experimentally determined bulk modulus of high pressure polymorph of $\mathrm{Mg}\left(\mathrm{BH}_{4}\right)_{2}$ is in a good agreement with the theoretically predicted value for $\operatorname{Mg}\left(\mathrm{AlH}_{4}\right)_{2}$ with the $P-3 m$ ltype structure $\left(\mathrm{K}_{0}=10.87 \mathrm{GPa}, \mathrm{K}_{0}^{\prime}=5.7\right)$ [78]. Low value of $\mathrm{K}_{0}$ suggests very high compressibility for the high pressure polymorph of $\operatorname{Mg}\left(\mathrm{BH}_{4}\right)_{2}$. To our knowledge this is the lowest bulk modulus reported for metal borohydrides, eg. $\mathrm{K}_{0}=19.9-31.1 \mathrm{GPa}$ for three different phases of $\mathrm{NaBH}_{4}[84$, 87], $\mathrm{K}_{0}=14.4-26 \mathrm{GPa}$ for three different phases of $\mathrm{LiBH}_{4}$ and $\mathrm{K}_{0}=16.8 \mathrm{GPa}$ for $\mathrm{KBH}_{4}$ $[79,87]$.

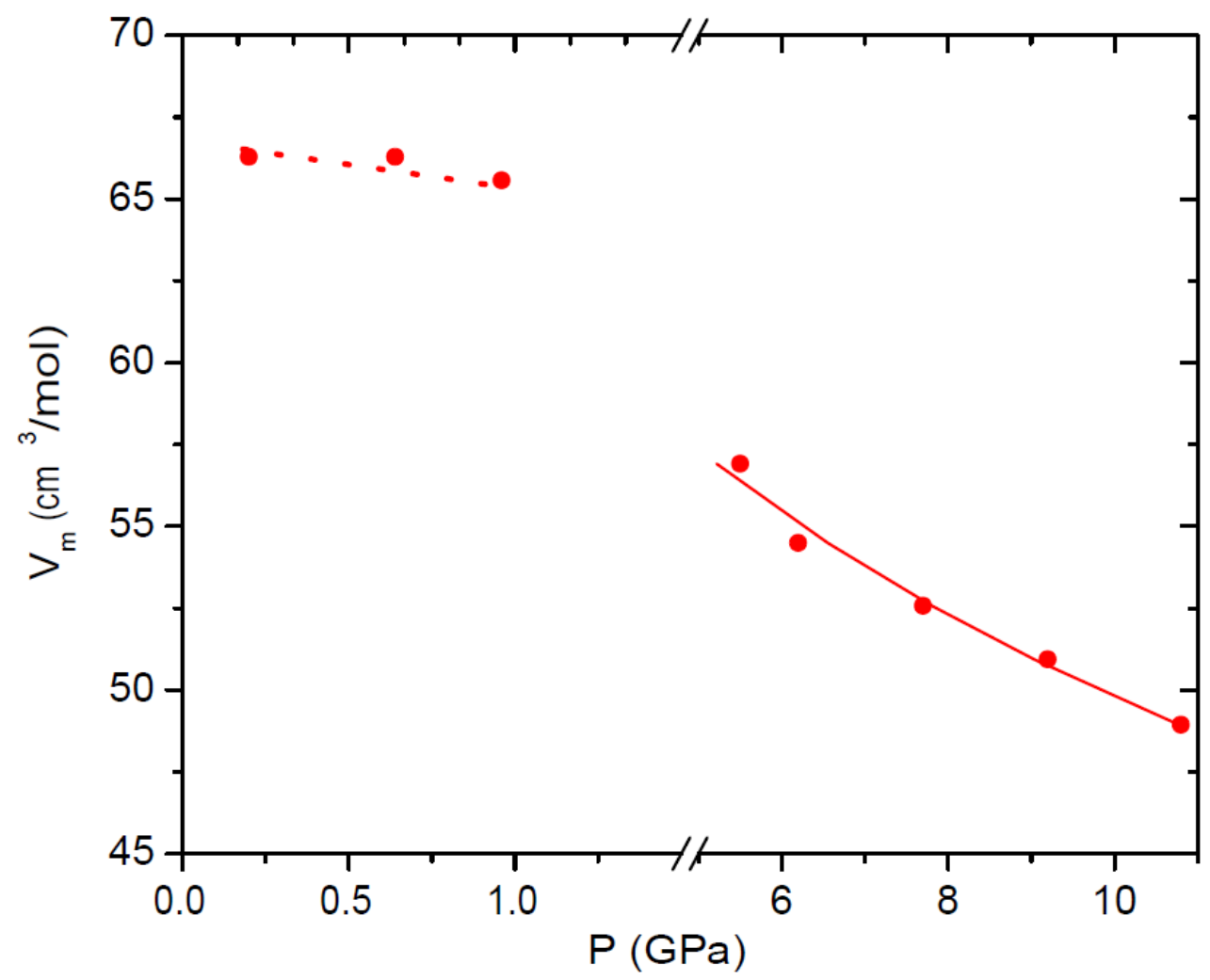

Figure 5. 3: Pressure dependence of volume (circles) for the high pressure phase of $\mathrm{Mg}\left(\mathrm{BH}_{4}\right)_{2}$, during compression, fitted with third order Birch- Murnaghan EoS (line). 


\subsubsection{Raman spectroscopy measurements}

\subsubsection{As synthesized $\mathrm{Mg}\left(\mathrm{BH}_{4}\right)_{2}$}

Raman spectra were collected with and without mineral oil as pressure transmitting medium to compare the results and observed similar results. Raman spectrum collected at ambient conditions from $\mathrm{Mg}\left(\mathrm{BH}_{4}\right)_{2}$ was in good agreement with the literature data [153]. The Raman spectra show liberational and translational modes in the range 50-500 $\mathrm{cm}^{-1}$, bending deformation modes $\sim 1100-1400 \mathrm{~cm}^{-1}$ and B-H stretching modes around $2250 \mathrm{~cm}^{-1}$. The strong bending and stretching modes suggest a strong network of $\mathrm{Mg} \ldots \mathrm{H}-\mathrm{B}$ bonds and a complex structure for $\mathrm{Mg}\left(\mathrm{BH}_{4}\right)_{2}$. Figure 5.4 shows the Raman spectra obtained during compression up to $\sim 21 \mathrm{GPa}$ for various modes. Spectrum recorded at $\sim 1.1 \mathrm{GPa}$ shows coexistence of both ambient and high pressure phase. The B$\mathrm{H}$ stretching modes start splitting around 2.5 GPa, and around 14.4 GPa a shift in Raman vibrational frequency of $\mathrm{B}-\mathrm{H}$ bending modes is observed. These changes can be attributed to phase transitions.

The weakening of B-H bending modes observed above $9.5 \mathrm{GPa}$ has occurred due to existence of pressure gradient in the sample and expect that laser heating has less effect on intensity of Raman modes in this experiment [159]. The phase transition observed around 14.4 GPa is completely reversible around 4.5 GPa upon decompression. The spectrum of compressed sample after releasing pressure shows close similarity to the spectra in the range 2.5-14.4 GPa during compression which suggest that the phase transition of $\mathrm{Mg}\left(\mathrm{BH}_{4}\right)_{2}$ at $2.5 \mathrm{GPa}$ is irreversible. Figure 5.5 shows Raman spectra collected at various pressures during decompression for bending and stretching modes. 
Decompression spectra of other modes are not shown in the figure due to their very low intensity.

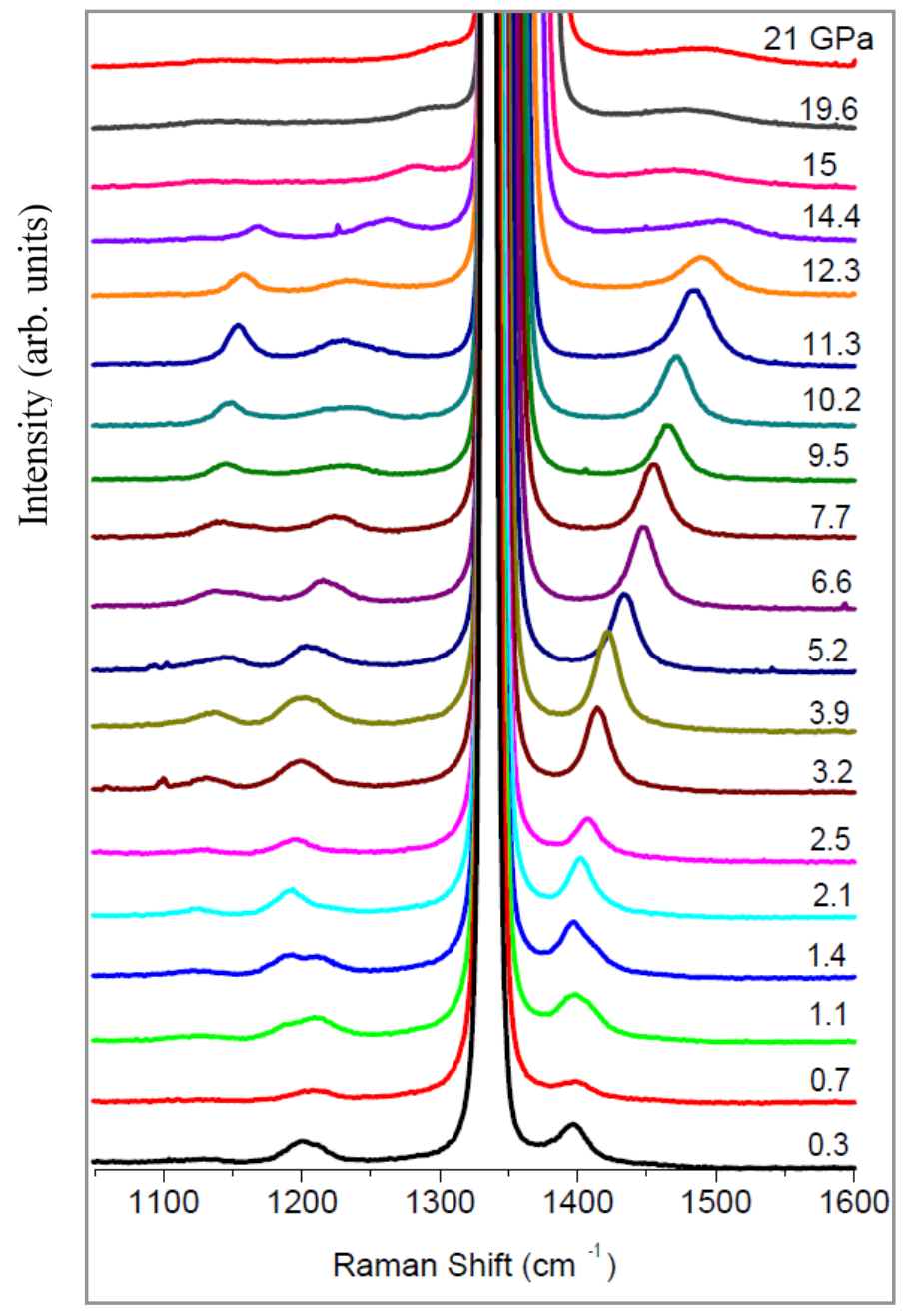

(a)

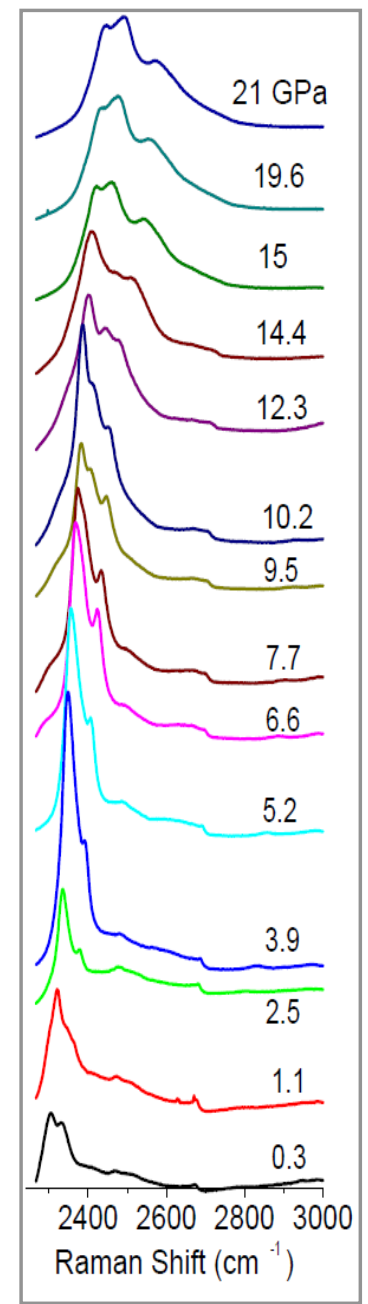

(b)

Figure 5. 4: The Raman spectra of $\mathrm{Mg}\left(\mathrm{BH}_{4}\right)_{2}$ collected during compression up to $\sim 21$

GPa at some selected pressures; (a) B-H bending modes, (b) B-H stretching modes. The peak around $1350 \mathrm{~cm}^{-1}$ is because of diamond in DAC.

A comparative study of compression and decompression data confirms a difference in structure between the compressed and non compressed sample which 
support the irreversible phase transition observed in XRD analysis. Therefore from combined XRD and Raman spectroscopy experiments it may conclude that the initial high pressure phase transition in $\mathrm{Mg}\left(\mathrm{BH}_{4}\right)_{2}$ occurs around $2.5 \mathrm{GPa}$ and it is stable up to $14 \mathrm{GPa}$.

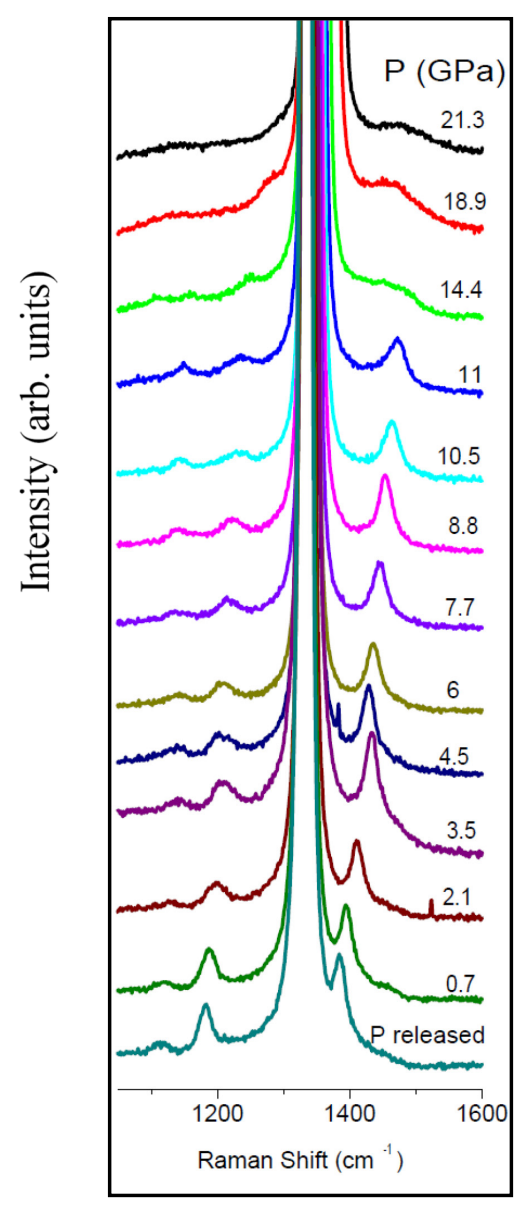

(a)

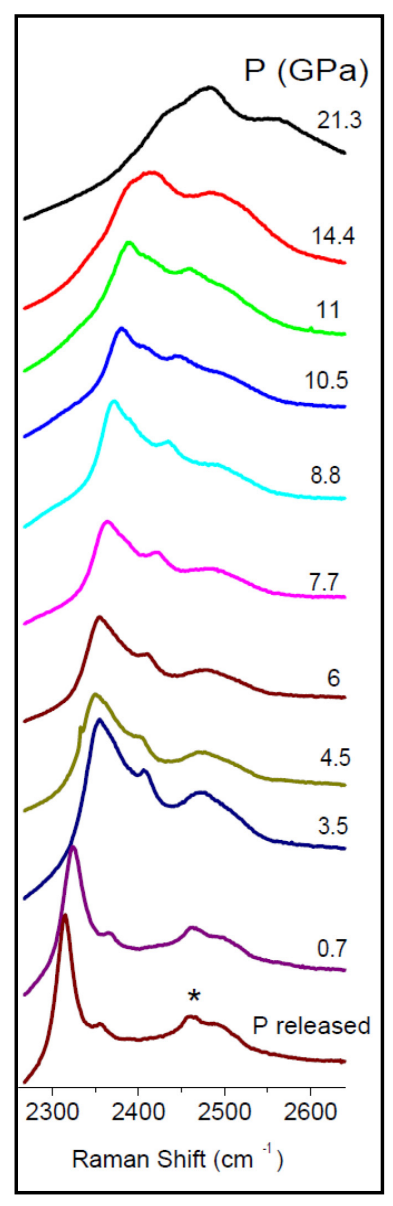

(b)

Figure 5. 5: The Raman spectra of $\mathrm{Mg}\left(\mathrm{BH}_{4}\right)_{2}$ collected during decompression to ambient pressure; (a) B-H bending modes, (b) B-H stretching modes. Peak marked with asterisks is due to impurities in diamond. 
A small amount of $\mathrm{H}_{2}$ was released in the experiment conducted without using mineral oil which can be attributed to the reaction of small amount of sample with the moisture in the gasket chamber. Table 5.1 shows change in Raman vibrational frequency as a function of pressure $(\mathrm{d} v / \mathrm{dp})$ for bending as well as liberational and translational modes during compression of as prepared $\mathrm{Mg}\left(\mathrm{BH}_{4}\right)_{2}$. In all these cases a significant change in slopes is observed after phase transitions. During decompression a shift in $\mathrm{d} v / \mathrm{dp}$ was observed around $11 \mathrm{GPa}$ for all the modes.

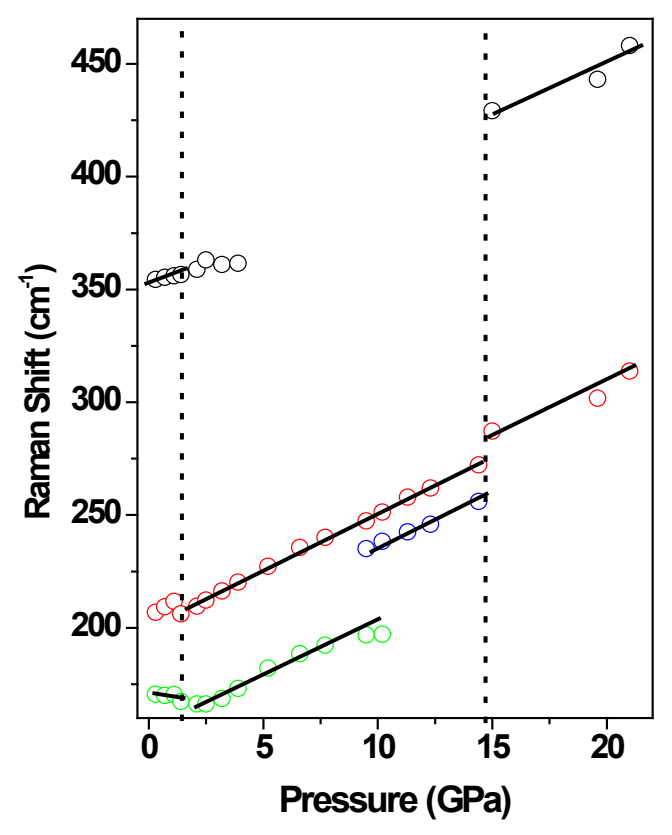

(a)

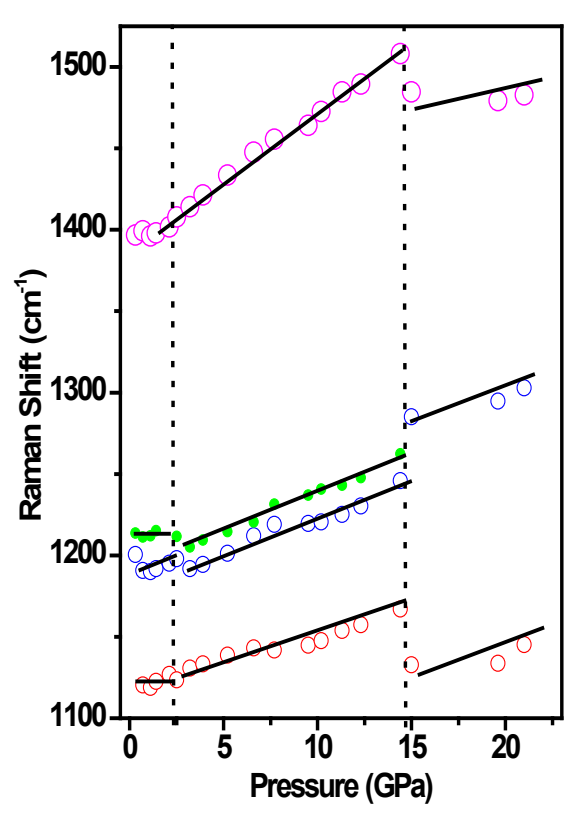

(b)

Figure 5. 6: Change in vibrational frequency with pressure for as prepared $\operatorname{Mg}\left(\mathrm{BH}_{4}\right)_{2}$ during compression; (a) liberational and translational modes, (b) B-H bending modes. The pressures corresponding to change in vibrational modes during compression is marked by grid lines parallel to $\mathrm{Y}$ axis. 


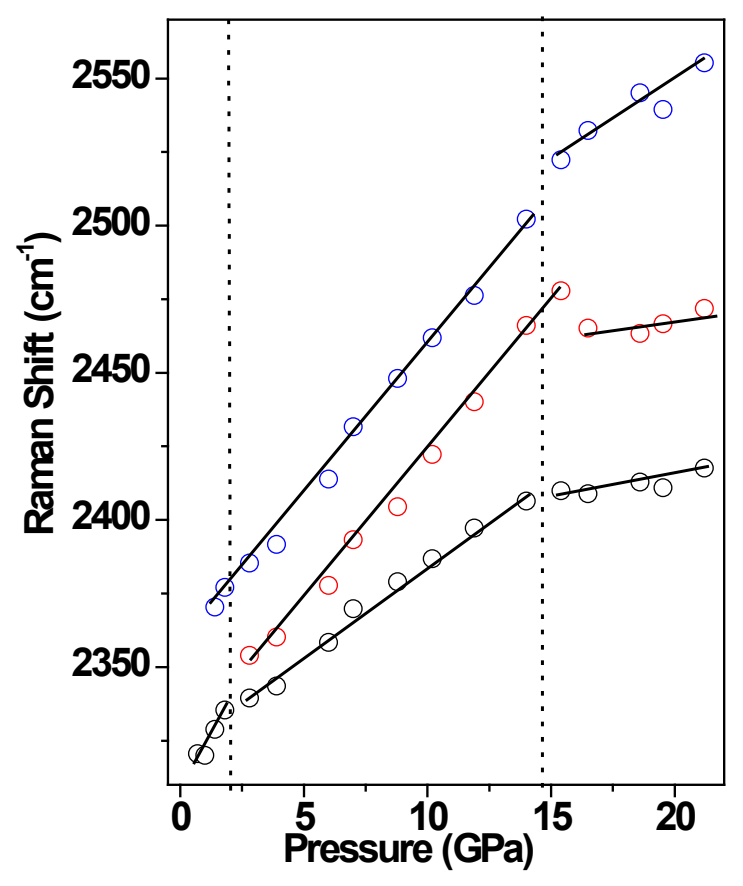

(a)

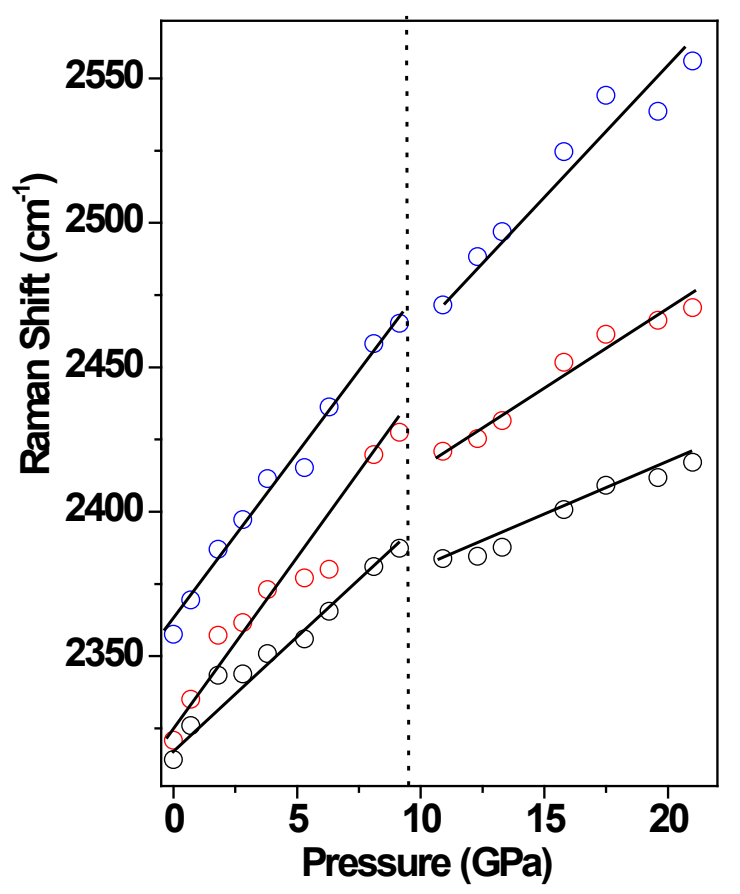

(b)

Figure 5. 7: Change in vibrational frequency of B-H stretching modes with pressure for as prepared $\mathrm{Mg}\left(\mathrm{BH}_{4}\right)_{2}$; (a) during compression, (b) during decompression. The pressures corresponding to change in vibrational modes during compression is marked by grid lines parallel to Y axis.

The difference in $\mathrm{d} v / \mathrm{dP}$ values of stretching modes (Table 5.2) during compression and decompression also confirms the irreversibility of phase transition in of $\mathrm{Mg}\left(\mathrm{BH}_{4}\right)_{2}$ as inferred from XRD analysis. The thermodynamically stable high pressure phase has to be further investigated to identify its potential as a hydrogen storage material in terms of kinetic and volumetric aspects. 
Table 5. 1: Raman frequency shifts as a function of pressure for liberational, translational and bending modes from fitting the compression data of as synthesized sample.

\begin{tabular}{|c|c|c|c|}
\hline \multirow{2}{*}{$\begin{array}{l}\text { Modes } \\
1 \operatorname{atm}\left(\mathrm{cm}^{-1}\right)\end{array}$} & \multicolumn{3}{|c|}{$\mathrm{dv} / \mathrm{dp}\left(\mathrm{cm}^{-1} \mathrm{GPa}^{-1}\right)$ at $\mathrm{P}$ - ranges: } \\
\hline & 0-2.5 GPa & 2.5-14.5 Gpa & 14.5-22 GPa \\
\hline 170 & -2.61 & 4.24 & \\
\hline 200 & & 4.19 & \\
\hline 210 & 6.12 & 5.06 & 4.13 \\
\hline 350 & 2.32 & & 4.38 \\
\hline 1120 & 5.26 & 3.08 & 1.6 \\
\hline 1200 & 0.48 & 4.38 & 2.76 \\
\hline 1210 & -0.35 & 4.91 & \\
\hline 1400 & 2.2 & 8.27 & 1.45 \\
\hline
\end{tabular}

Table 5. 2: Raman frequency shifts as a function of pressure for B- $\mathrm{H}$ stretching modes during both compression and decompression of as prepared $\mathrm{Mg}\left(\mathrm{BH}_{4}\right)_{2}$.

\begin{tabular}{|c|c|c|c|}
\hline Modes & \multicolumn{3}{|c|}{$\mathrm{dv} / \mathrm{dp}\left(\mathrm{cm}^{-1} \mathrm{GPa}^{-1}\right)$ at different P- ranges } \\
\hline Compression & 0- 2.5 GPa & 2.5-14.5 GPa & 14.5- $22 \mathrm{GPa}$ \\
\hline 2300 & 19.75 & 6.17 & 3.48 \\
\hline 2340 & 10.13 & 9.59 & 4.24 \\
\hline 2360 & 13.71 & 11.49 & 5.47 \\
\hline Decompression & 3.5- 0 GPa & 11- 4.5 GPa & 22- $14.4 \mathrm{GPa}$ \\
\hline 2300 & 9.78 & 5.71 & 6.52 \\
\hline 2340 & 19.09 & 10.34 & 9.01 \\
\hline \multirow[t]{2}{*}{2360} & 13.17 & 8.86 & 10.97 \\
\hline & 0- 9 GPa & 11- 21 GPa & \\
\hline 2300 & 7.26 & 3.6 & \\
\hline 2340 & 10.55 & 5.33 & \\
\hline 2360 & 11.51 & 8.12 & \\
\hline
\end{tabular}

\subsubsection{The high temperature phase of $\mathrm{Mg}\left(\mathrm{BH}_{4}\right)_{2}$}

The ambient hexagonal phase of $\mathrm{Mg}\left(\mathrm{BH}_{4}\right)_{2}$ transforms to an orthorhombic- $\mathrm{Pmc} 2_{1}$ structure above $180{ }^{\circ} \mathrm{C}$ and the phase transition is irreversible [95]. To compare the high pressure behavior of as prepared sample and the high temperature phase, Raman spectroscopic studies of the latter have also been carried out. The high temperature phase 
is synthesized by heating the sample loaded in DAC in an oven at $200{ }^{\circ} \mathrm{C}$ for $18 \mathrm{hrs}$ and the pressure was close to $1 \mathrm{~atm}$. Figure 5.8a shows comparison of Raman spectra at the stretching mode region for as prepared and heated samples which indicate a high temperature phase has been formed. High pressure Raman spectroscopic studies were carried out on the high temperature phase after cooling down to room temperature. The high temperature phase is found to be stable up to $2 \mathrm{GPa}$. Figure $5.8 \mathrm{~b}$ shows the pressure dependence of B-H stretching modes of high temperature phase of $\mathrm{Mg}\left(\mathrm{BH}_{4}\right)_{2}$.

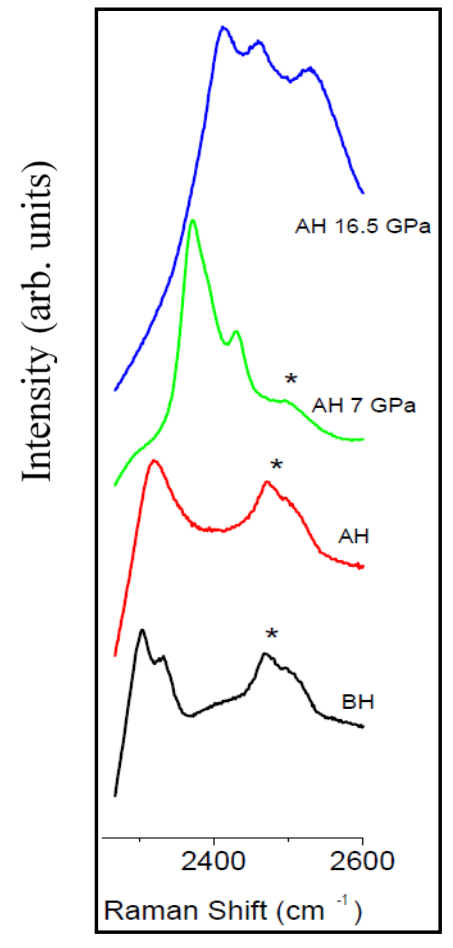

(a)

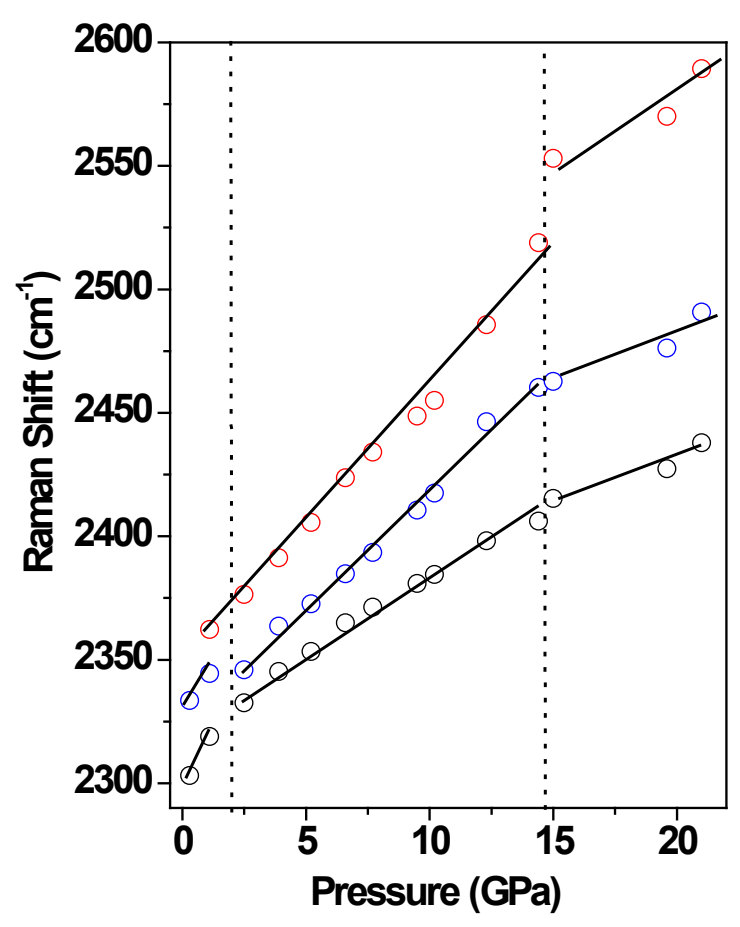

(b)

Figure 5. 8: (a) Raman spectra of as prepared $\operatorname{Mg}\left(\mathrm{BH}_{4}\right)_{2}$ in comparison with the high temperature phase during compression; peak marked with asterisks is due to impurities in diamond; $\mathrm{BH}-$ before heating (as synthesized); $\mathrm{AH}-$ after heating (high temperature phase). (b) Change in B-H stretching modes with pressure for the high temperature phase. 
In Raman spectroscopic study, significant mode changes were observed around 2 GPa and above $14 \mathrm{GPa}$. From the Raman spectra it is obvious that high pressure phase of both as prepared sample and the heated sample is of similar structure as both samples show same frequency shift in the range 2.5-14 GPa. However, there is a significant difference between spectra of both phases above $14 \mathrm{GPa}$. The disordering of highly compressible $\mathrm{B}-\mathrm{H}$ bonds in the $\mathrm{BH}_{4}{ }^{-}$tetrahedra can be resulting in the phase transitions.

Table 5. 3: Raman frequency shifts as a function of pressure for B-H stretching modes during both compression and decompression for the high temperature phase of $\operatorname{Mg}\left(\mathrm{BH}_{4}\right)_{2}$.

\begin{tabular}{lccc}
\hline $\begin{array}{l}\text { Modes } \\
\left(\mathbf{c m}^{-1}\right)\end{array}$ & \multicolumn{1}{c}{$\mathbf{d v} / \mathbf{d p}\left(\mathbf{c m}^{-1} \mathbf{G P a}^{-\mathbf{1}}\right)$ at range of pressures: } \\
\hline Compression & $\mathbf{0 - 2} \mathbf{~ G P a}$ & $\mathbf{2 . 8 - 1 4} \mathbf{~ G P a}$ & $\mathbf{1 4 - 2 2} \mathbf{~ G P a}$ \\
\hline 2300 & 14.6 & 6.23 & 1.24 \\
2340 & 16.7 & 10.02 & 3.25 \\
2360 & - & 10.54 & 5.07 \\
Decompression & $\mathbf{1 . 8 - 0 G P a}$ & $\mathbf{2 . 8 - 9} \mathbf{~ G P a}$ & $\mathbf{2 2 - 1 1} \mathbf{~ G P a}$ \\
2300 & 16.14 & 6.98 & 3.61 \\
2340 & 20.18 & 10.51 & 5.33 \\
2360 & 16.33 & 10.96 & 8.11 \\
\hline
\end{tabular}

\subsubsection{High pressure phase of $\mathrm{Mg}\left(\mathrm{BH}_{4}\right)_{2}$}

The experiments show that as synthesized $\mathrm{Mg}\left(\mathrm{BH}_{4}\right)_{2}$ undergoes an irreversible high pressure transition above $2.5 \mathrm{GPa}$. Therefore it is important to study the decomposition temperature of this polymorph to check if it is a kinetically destabilized phase. The high pressure phase was prepared by pressurizing as synthesized sample loaded in a DAC up to 5.6 GPa. The high pressure phase was retained when the pressure 
was released which can be noticed from Figure 5.9. Then it was heated at various temperatures up to $375{ }^{\circ} \mathrm{C}$ using a gas blower, as closed in the DAC to avoid reaction with moisture. Neither phase transition nor decomposition was observed up to this temperature. The hexagonal phase was observed to undergo phase transition above 180 ${ }^{\circ} \mathrm{C}$ and then decompose in two steps, at 290 and $317{ }^{\circ} \mathrm{C}$, respectively [37, 38]. Further studies have to be conducted to determine the decomposition temperature of the high pressure phase and also to destabilize this compound by catalytic addition.

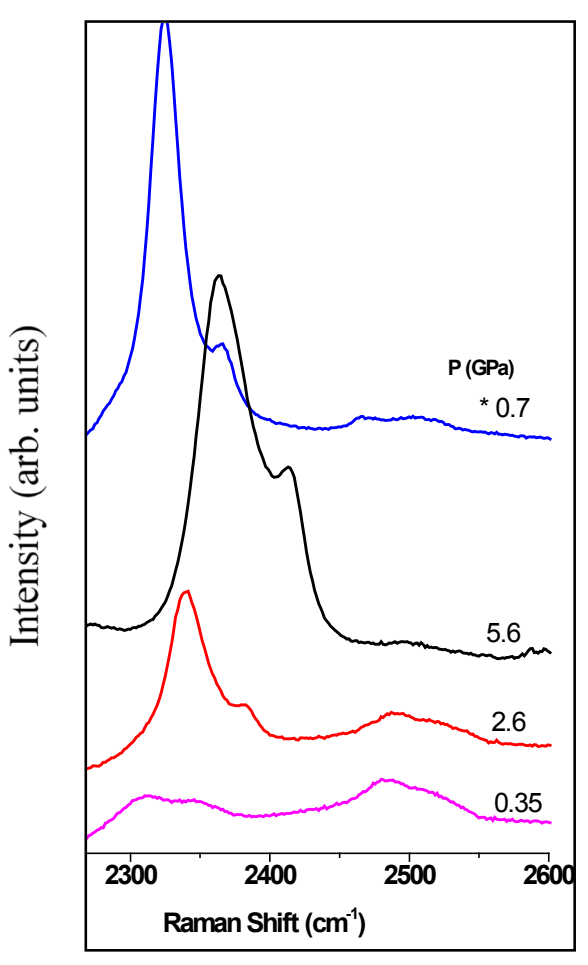

(a)

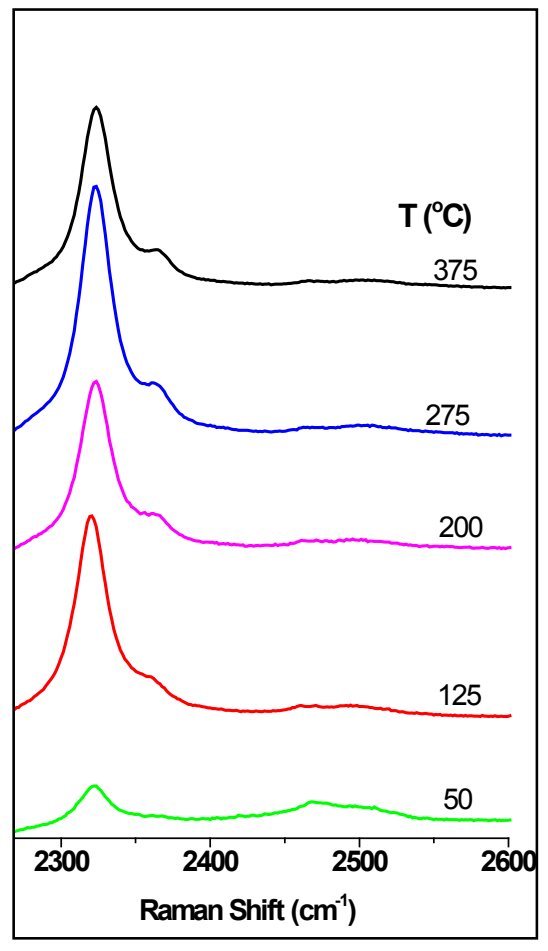

(b)

Figure 5. 9: Raman spectra of $\operatorname{Mg}\left(\mathrm{BH}_{4}\right)_{2}$; (a) as synthesized phase in comparison with the high pressure phase during compression at some selected pressures,(b) high pressure phase up to $375^{\circ} \mathrm{C}$ at some selected temperatures. 


\section{CHAPTER 6. HIGH PRESSURE INVESTIGATION OF Ca(BH $\left.{ }_{4}\right)_{2}$}

\subsection{Introduction}

Calcium borohydride, $\mathrm{Ca}\left(\mathrm{BH}_{4}\right)_{2}$, has a theoretical hydrogen storage capacity of 11.48 wt \%. Complex hydrides have high gravimetric storage capacities and among them $\mathrm{Ca}\left(\mathrm{BH}_{4}\right)_{2}$ and $\mathrm{Mg}\left(\mathrm{BH}_{4}\right)_{2}$ are promising materials because of the possibility of reversible hydrogen storage [151]. In a proposed dehydrogenation reaction, hydrogen release of 9.6 wt $\%$ is possible from $\mathrm{Ca}\left(\mathrm{BH}_{4}\right)_{2}$ with $\mathrm{CaH}_{2}$ and $\mathrm{CaB}_{6}$ as decomposition products [160]. The thermal decomposition behavior of $\mathrm{Ca}\left(\mathrm{BH}_{4}\right)_{2}$ was investigated under hydrogen pressure by Kim et al. [39], and under vacuum or inert gas flow by Aoki et al. [161]. The results show that $\mathrm{Ca}\left(\mathrm{BH}_{4}\right)_{2}$ undergoes transformation from an orthorhombic to a tetragonal structure below $250{ }^{\circ} \mathrm{C}$ with $5.9 \%$ hydrogen release. The structural investigations at different temperatures and pressures help to identify various polymorphs of the material which may have better thermodynamic stability. $\mathrm{Ca}\left(\mathrm{BH}_{4}\right)_{2}$ exhibits polymorphism depending on the synthesis and processing conditions and also transforms to different phases at various temperatures [96, 98, 151, 162-164]. Filinchuk et al. [96] observed three polymorphs of $\mathrm{Ca}\left(\mathrm{BH}_{4}\right)_{2}$ in the temperature range $30-600{ }^{\circ} \mathrm{C}$ which are of orthorhombic- $\alpha$ (F2dd), tetragonal- $\alpha^{\prime}$ (I-42d) and tetragonal- $\beta$ (P-4) structures. Below $300{ }^{\circ} \mathrm{C}, \alpha$ and ' $\alpha$ polymorphs completely transforms to $\beta$ phase. The second order transition from $\alpha$ to 'ais found to be dependent on heating rate/ sample synthesis and processing conditions. Two more possible structures of $\alpha$ phase are $\mathrm{C} 2 / \mathrm{c}$ and Fddd suggested by Majzoub et al. [163], and Miwa et al. [160], respectively. Buchter et al. [98], and Lee et al. [162], independently indexed tetragonal phase in $\mathrm{P} 4_{2} / \mathrm{m}$ space group 
which seems to be more reliable than the P-4 structure, according to the results of this study. An orthorhombic $(\gamma)$ phase of space group pbca was observed by Riktor et al. [164] which transforms to $\beta$ phase on heating to $290{ }^{\circ} \mathrm{C}$ and again to an unknown phase $\delta$ around $330^{\circ} \mathrm{C}$. Catalyst addition is also found to be influencing the formation of different phases in $\mathrm{Ca}\left(\mathrm{BH}_{4}\right)_{2}$ by Ronnebro et al. [151].

Majzoub et al.[163] observed formation of $\sim 50 \%$ of the $\beta$ phase after mechanical milling of $\alpha-\mathrm{Ca}\left(\mathrm{BH}_{4}\right)_{2}$ which was believed to be because of the combined effect of pressure and temperature. They calculated the bulk modulus of various polymorphs of $\mathrm{Ca}\left(\mathrm{BH}_{4}\right)_{2}$ using first principles method and it was $\sim 15 \mathrm{GPa}$. The same group also predicted transition of $\alpha$ to $\beta$ phase at $5.3 \mathrm{GPa}$. Even though there is much theoretical and high temperature work, high pressure experimental study on $\mathrm{Ca}\left(\mathrm{BH}_{4}\right)_{2}$ is not found in the current literature. Factors affecting the polymorphism of $\mathrm{Ca}\left(\mathrm{BH}_{4}\right)_{2}$ is not yet known completely. In situ X-ray diffraction and Raman spectroscopic studies on $\mathrm{Ca}\left(\mathrm{BH}_{4}\right)_{2}$ at high pressures were carried out to understand the structural stability of its polymorphs and to verify the results of theoretical calculations.

\subsection{Experimental Details}

\subsubsection{Synthesis}

Powder sample of $\mathrm{Ca}\left(\mathrm{BH}_{4}\right)_{2}$ was synthesized by heating $\mathrm{CaCl}_{2}$ and $\mathrm{NaBH}_{4}$ in tetrahydrofuran (THF) at $80{ }^{\circ} \mathrm{C}$. The obtained $\mathrm{Ca}\left(\mathrm{BH}_{4}\right)_{2} \cdot 2 \mathrm{THF}$ was dried at various temperatures and under vacuum for long time to remove THF. This process results in the formation of two polymorphs of $\mathrm{Ca}\left(\mathrm{BH}_{4}\right)_{2}$ in orthorhombic $(\alpha)$ and tetragonal $(\beta)$ structures. 


\subsubsection{High pressure micro X-ray diffraction}

Using an argon-filled glove box, this sample was loaded into the Mao-Bell type DAC with a diamond culet size of $400 \mu \mathrm{m}$. The sample chamber was a stainless steel gasket with hole of size $\sim 190 \mu \mathrm{m}$ and thickness of $50 \mu \mathrm{m}$. Both compression and decompression data were collected at room temperature. The ruby fluorescence method was used for pressure determination. Due to high reactivity of $\mathrm{Ca}\left(\mathrm{BH}_{4}\right)_{2}$ any kind of pressure transmitting medium was avoided. High pressure diffraction studies were conducted at Sector 16-IDB, HPCAT, Advanced Photon Source of Argonne National Laboratory using X-rays of wavelength $\sim 0.3681 \AA$ and Mar345 image plate detector. The micro XRD experiment was repeated at the X17C beam line at NSLS, Brookhaven National Laboratory which facilitating radiation of $\lambda=0.4066 \AA$.

\subsection{Results and Discussion}

\subsubsection{X-ray diffraction}

Figure 6.1 shows synchrotron $\mathrm{X}$-ray diffraction patterns of $\mathrm{Ca}\left(\mathrm{BH}_{4}\right)_{2}$ collected at ambient conditions and the results of Rietveld analysis. The analysis shows that sample is a homogeneous mixture of $\alpha$ and $\beta$ polymorphs. The $\alpha-\mathrm{Ca}\left(\mathrm{BH}_{4}\right)_{2}$ is indexed with orthorhombic- F2dd structure and the lattice parameters are $a=8.82(7) \AA, b=13.05(10)$ $\AA, \mathrm{c}=7.404(5) \AA$ and $\mathrm{V}=851.8(10) \AA^{3}$ which agree well with the previous report [96]. All the previously reported tetragonal structures for $\beta$ phase were checked and best match was obtained when space group $\mathrm{P} 4_{2} / \mathrm{m}$ is used. The lattice parameters for $\beta-\mathrm{Ca}\left(\mathrm{BH}_{4}\right)_{2}$ is found to be a $=6.88(4) \AA, c=4.32(5) \AA$ and $V=204.82(18) \AA^{3}$. This result is in 
agreement with that reported by Buchter et al. [98], and Lee et al.[162], but contradict with P-4 structure proposed by Filinchuk et al. [96], and Majzoub et al. [163].

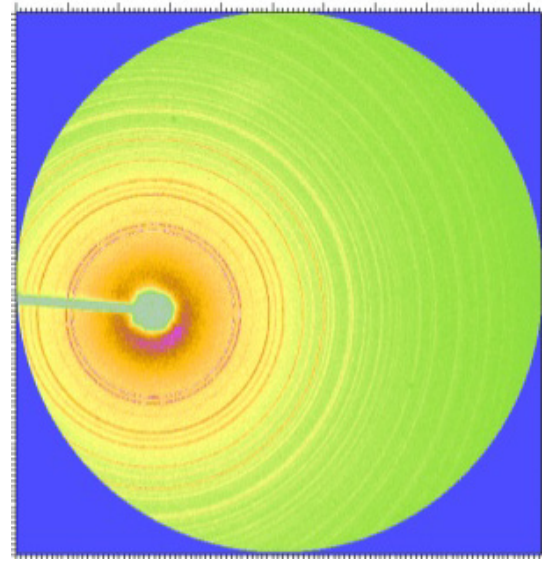

(a)

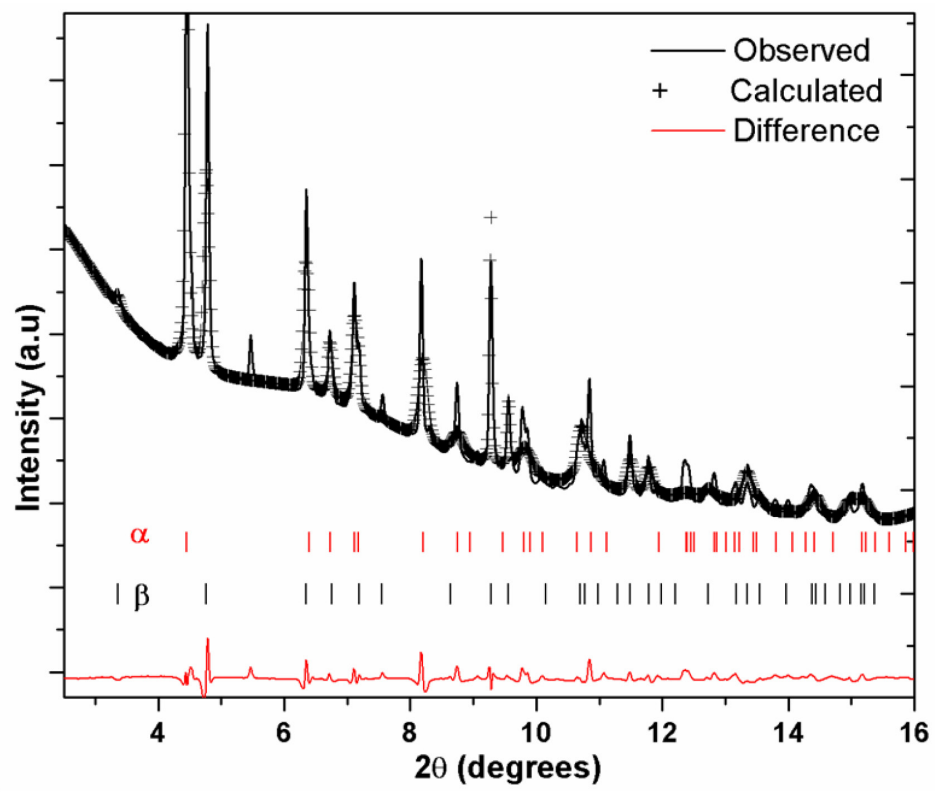

(b)

Figure 6. 1: Synchrotron $X$-ray diffraction pattern of the sample containing $\alpha$ and $\beta$ polymorphs of $\mathrm{Ca}\left(\mathrm{BH}_{4}\right)_{2}$ at ambient conditions; (a) Debye ring, (b) integrated plot with the results of Rietveld analysis.

Figure 6.2 shows picture of crystal structures of both $\alpha$ and $\beta$ polymorphs in F2dd $(\mathrm{Z}=8)$ and $\mathrm{P} 4_{2} / \mathrm{m}(\mathrm{Z}=2)$ space groups, respectively. The calculated molar volumes are 64.13 and $61.68 \mathrm{~cm}^{3} / \mathrm{mol}$ for $\alpha$ and $\beta$ phase respectively. Molar volume of $\beta$ phase is less than that of $\alpha-\mathrm{Ca}\left(\mathrm{BH}_{4}\right)_{2}$ implying a more close packed structure for the former. Figure 6.3 shows XRD patterns at various pressure during compression in a DAC up to $13 \mathrm{GPa}$ in comparison with a pattern obtained from decompressed sample. The disappearance of 
peaks corresponds to $\beta-\mathrm{Ca}\left(\mathrm{BH}_{4}\right)_{2}$ above $10 \mathrm{GPa}$ may corresponds to transition to a highly disordered phase as discussed later in Raman spectroscopy results.

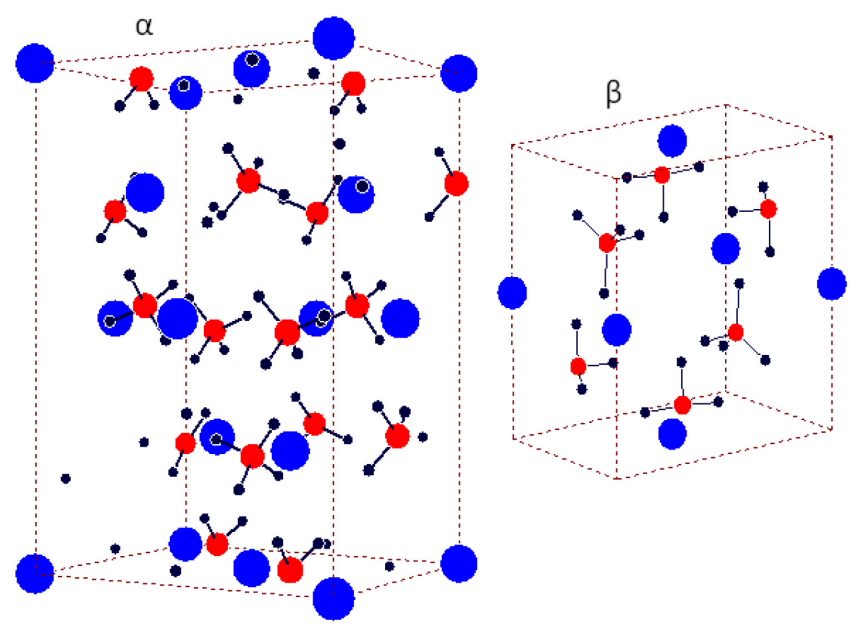

Figure 6. 2: The refined crystal structures of $\mathrm{Ca}\left(\mathrm{BH}_{4}\right)_{2}$ polymorphs; $(\alpha)$ orthorhombic and ( $\beta$ ) tetragonal- $\mathrm{P} 42 / \mathrm{m}$ phases. The atoms $\mathrm{Ca}, \mathrm{B}$ and $\mathrm{H}$ are represented by symbols of color blue, red, and black, respectively.

Upon decompression it is found that the transformation of $\beta$ phase is irreversible while $\alpha$ phase is stable in the pressure range studied. The calculated formation energies of $\alpha$ and $\beta$ phases are of the same order [98]. Therefore, both phases should coexist at similar thermodynamical conditions. The results indicate that above $10 \mathrm{GPa}, \alpha$ phase is more stable than the $\beta$ phase which is compatible with previous report that the $\alpha$ phase has a lower free energy than the $\beta-\mathrm{Ca}\left(\mathrm{BH}_{4}\right)_{2}$ [162]. The unit cell parameters for $\alpha$ phase at different pressures were obtained by Le Bail method. 


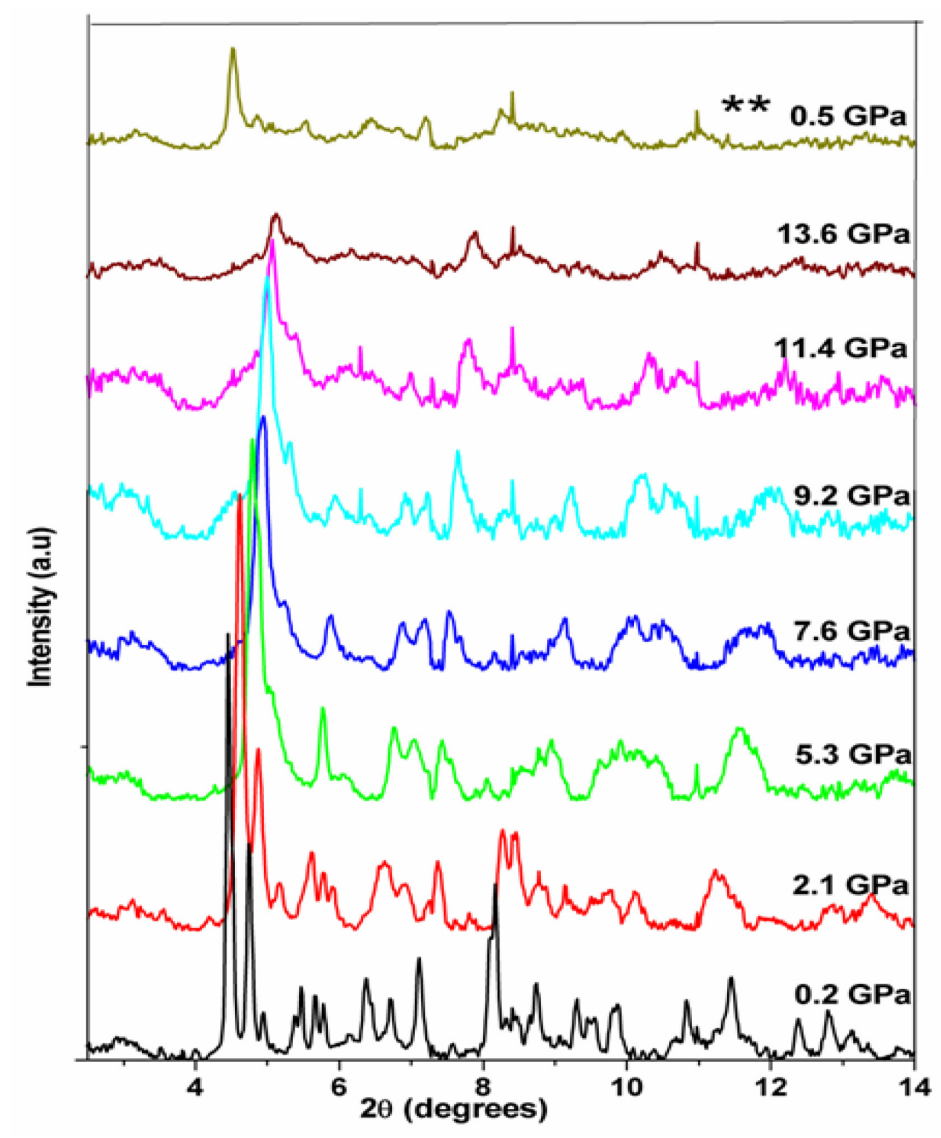

Figure 6. 3: Synchrotron $\mathrm{XRD}$ patterns of $\mathrm{Ca}\left(\mathrm{BH}_{4}\right)_{2}$; during compression up to $13.6 \mathrm{GPa}$ and after decompression to $0.5 \mathrm{GPa}($ marked $* *)$.

Pressure volume data obtained for $\alpha-\mathrm{Ca}\left(\mathrm{BH}_{4}\right)_{2}$ was fitted with third order BirchMurnaghan EoS (Figure 6.4) and its bulk modulus is found to be 22.95(4) GPa with $\mathrm{V}_{\mathrm{o}}=$ 866.06(18) $\AA^{3}$ and $\mathrm{K}_{\mathrm{o}}{ }^{\prime}=2.63(0.5)$. This is higher than the calculated bulk modulus for the polymorphs of $\mathrm{Ca}\left(\mathrm{BH}_{4}\right)_{2}$ by first-principles method which was $15 \mathrm{GPa}$ [163]. The compressibility of $\mathrm{Ca}\left(\mathrm{BH}_{4}\right)_{2}$ is of the order of many metal borohydrides $[96,97]$. Any $\alpha$ to $\beta$ phase transition was not observed in this experiment, around $5.3 \mathrm{GPa}$, as reported by Majzoub et al. [163]. 


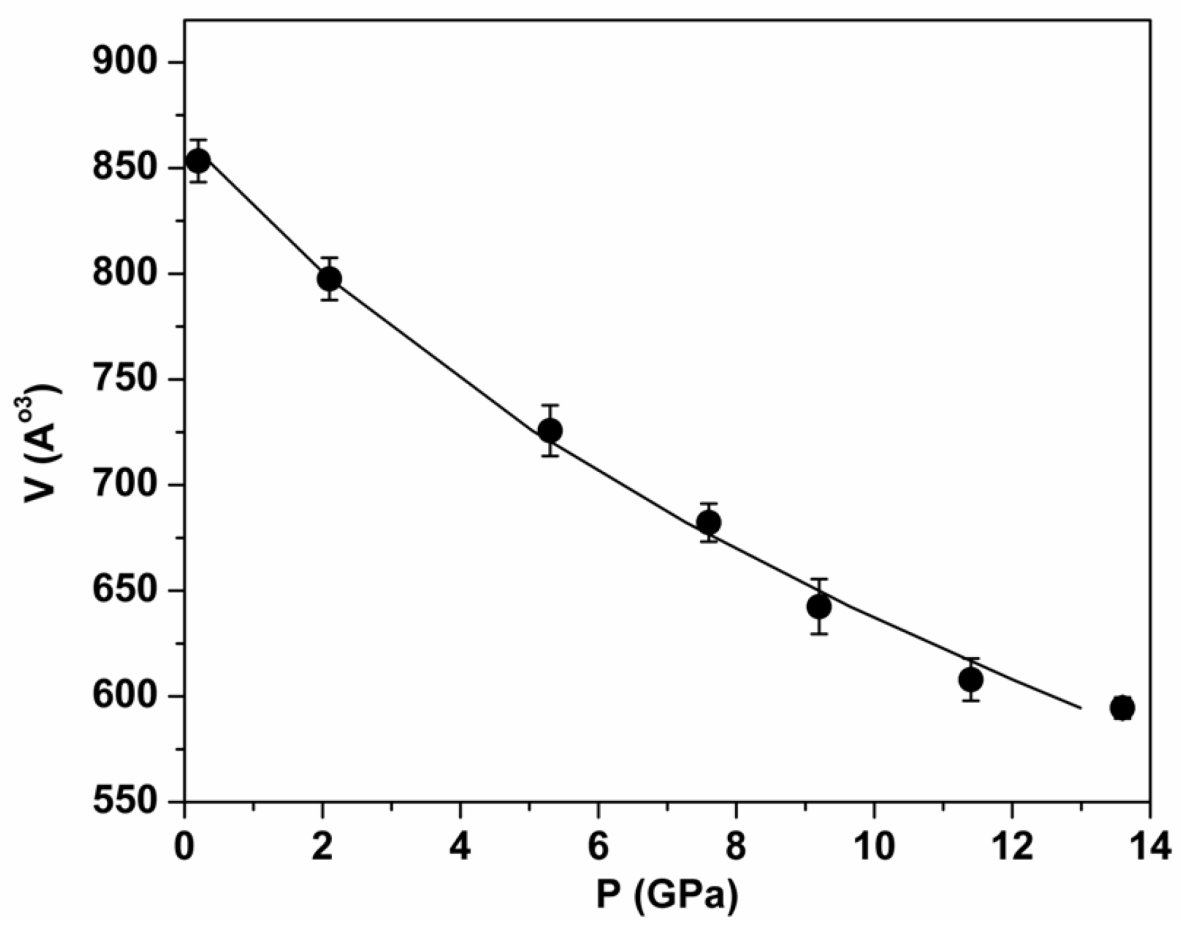

Figure 6. 4: Pressure dependence of the volume (circles) for $\alpha-\mathrm{Ca}\left(\mathrm{BH}_{4}\right)_{2}$ during compression, fitted with the third-order Birch-Murnaghan equation of state (line).

\subsubsection{Raman spectroscopy}

Figure 6.5 shows Raman spectrum collected at ambient conditions which confirms the presence of both $\alpha$ and $\beta-\mathrm{Ca}\left(\mathrm{BH}_{4}\right)_{2}$ phases in the sample in comparison with the previous reports $[160,163]$. The peaks corresponds to each phase is resolved with a Gaussian profile fitting. The analysis of $\mathrm{BH}_{4}^{-}$liberational $\left(50-550 \mathrm{~cm}^{-1}\right)$ and $\mathrm{B}-\mathrm{H}$ bending modes in the region $1000-1300 \mathrm{~cm}^{-1}$ are not included in Raman spectra at high pressures because of its weak intensity or its overlap with the Raman peak of diamond. 


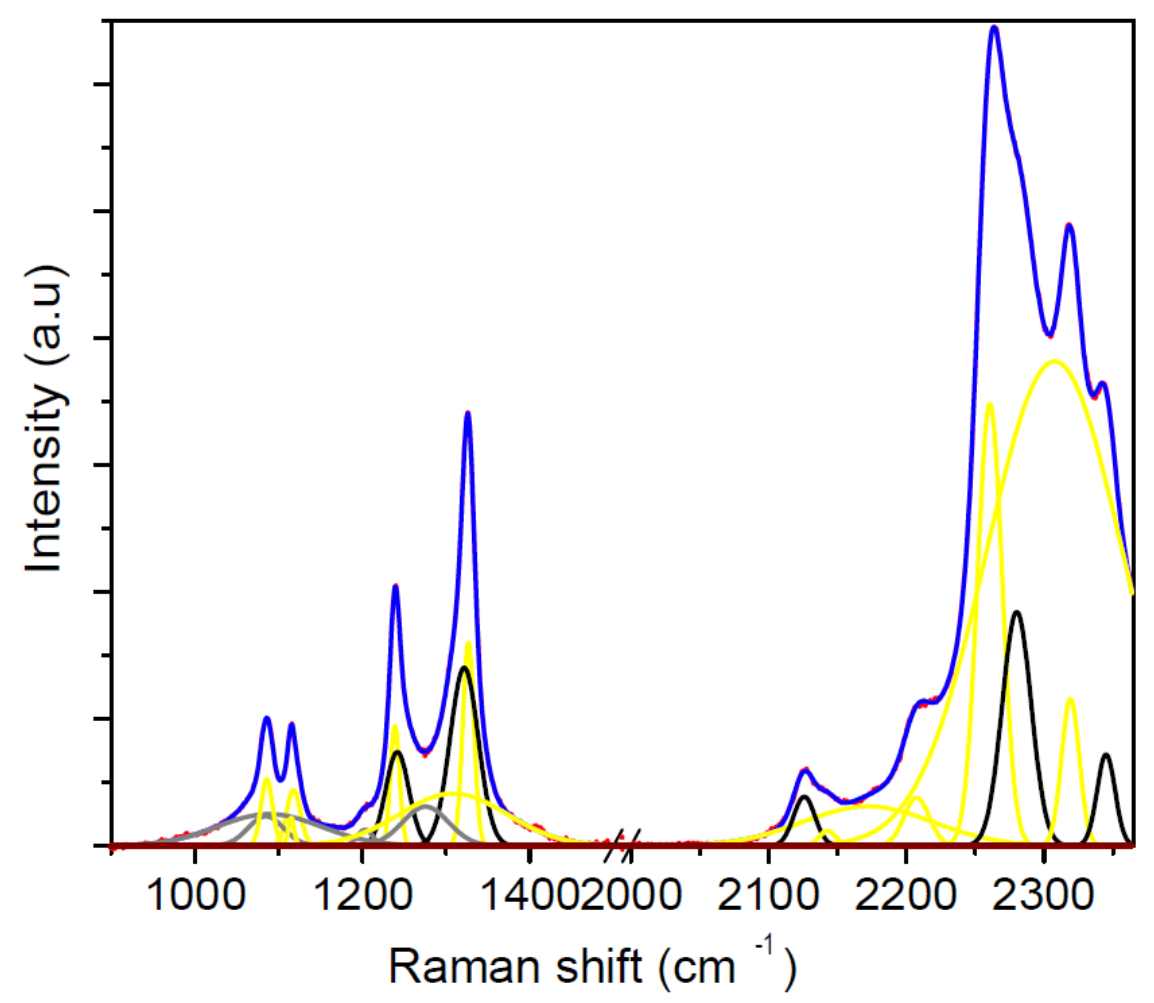

Figure 6. 5: Raman spectrum (blue line) of $\mathrm{Ca}\left(\mathrm{BH}_{4}\right)_{2}$ containing both $\alpha$ (yellow colored peaks) and $\beta$ (black colored peaks) polymorphs collected at ambient conditions.

Figure 6.6 shows the Raman spectra at various pressures in the region of B-H stretching modes (2250- $2400 \mathrm{~cm}^{-1}$ ) collected during compression and decompression. Raman spectrum obtained in the range 0.2- 10.2 GPa shows coexistence of the $\alpha$ and $\beta$ phases without any phase change. Above $10.2 \mathrm{GPa}$ there is a shift in Raman vibrational frequency of peaks corresponds to the $\alpha$ phase. While peaks of the $\beta$ phase disappears and a new peak originates around $2307 \mathrm{~cm}^{-1}$ at $10.2 \mathrm{GPa}$. This is much clear in the plot of variation of Raman shift with pressure (Figure 6.7). The analysis results implies a structural transition of the $\beta$ phase and occurrence of a hardening or reordering of $\mathrm{B}-\mathrm{H}$ bonds in the $\alpha$ - phase at high pressures. 


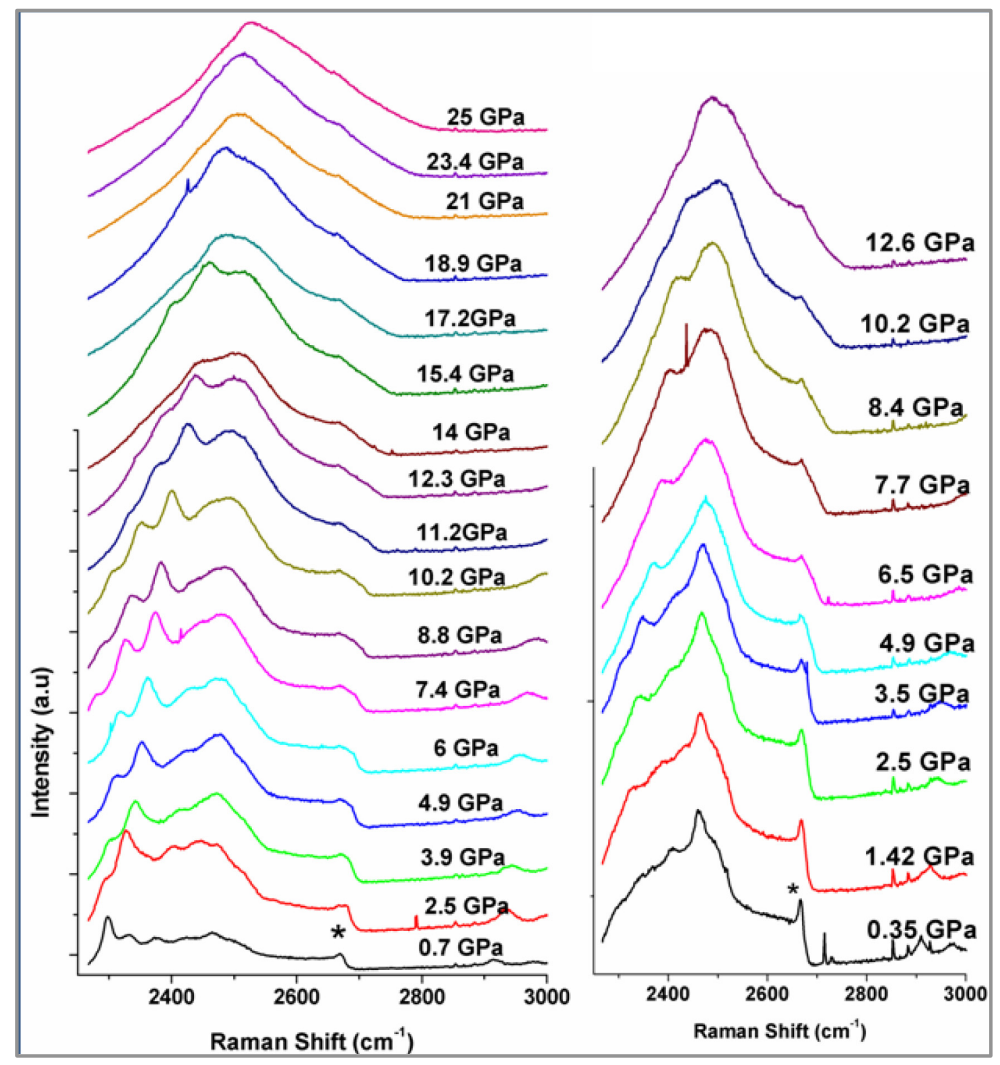

(a)

(b)

Figure 6. 6: Raman spectra of $\mathrm{Ca}\left(\mathrm{BH}_{4}\right)_{2}$; (a) during compression up to $25 \mathrm{GPa}$, (b) during decompression for B-H stretching modes. The peak (marked *) around $2650 \mathrm{~cm}^{-1}$ is because of diamond in the DAC.

On decompression, the behavior of the $\alpha$-phase is found to be reversible around $5 \mathrm{GPa}$ while that of the $\beta$ - phase is irreversible. Change in Raman vibrational frequencies as a function of pressure $(\mathrm{d} v / \mathrm{d} P)$ for $\mathrm{B}-\mathrm{H}$ stretching modes during compression of $\mathrm{Ca}\left(\mathrm{BH}_{4}\right)_{2}$ is given in Table 6.1. During decompression a frequency shift is observed around $5 \mathrm{GPa}$, but the slopes remain unchanged for the peaks corresponds to the $\alpha$ - 
$\mathrm{Ca}\left(\mathrm{BH}_{4}\right)_{2}$. On comparing Raman spectroscopic results and $\mathrm{XRD}$ results, it may conclude that the $\beta$ phase transforms to a highly disordered structure above $10.2 \mathrm{GPa}$.
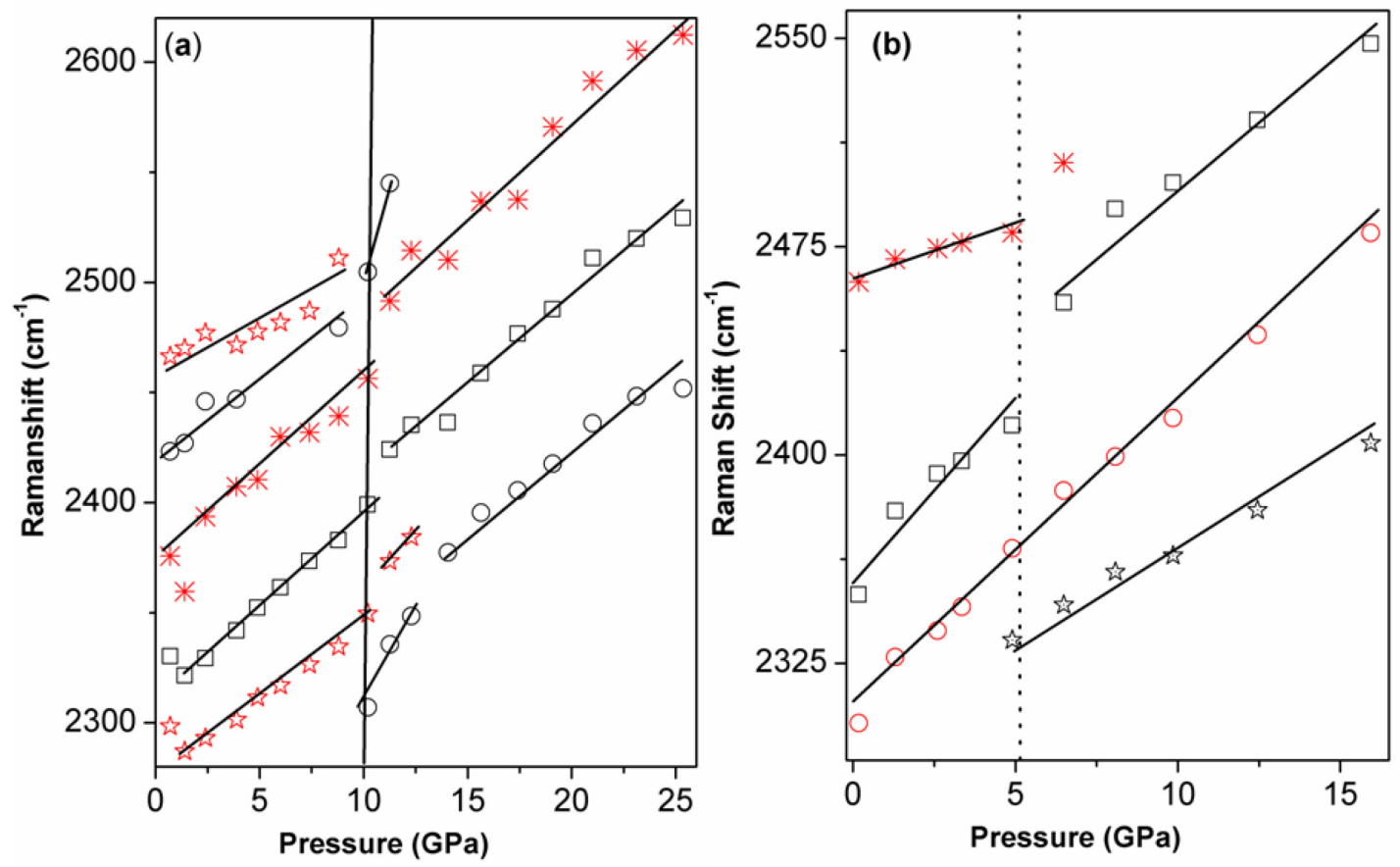

Figure 6. 7: Change in the Raman shift with pressure for B-H stretching modes of $\mathrm{Ca}\left(\mathrm{BH}_{4}\right)_{2}$; (a) during compression and (b) during decompression. The pressures corresponding to the change in vibrational modes are marked by grid lines parallel to the Y axis.

A partial decomposition of the $\mathrm{Ca}\left(\mathrm{BH}_{4}\right)_{2}$ sample leading to desorption of small amount of hydrogen was also observed in the Raman spectroscopic study $2.5 \mathrm{GPa}$, but the possible decomposition products were absent in the XRD pattern at this pressure. The decomposition may be caused by reaction with the small amount of moisture present in the gasket chamber. A comparison of spectra collected around $0.5 \mathrm{GPa}$ and after 
releasing pressure shows that $\alpha$ phase is unchanged which confirms the XRD results. It is obvious that there is a phase transition of $\beta-\mathrm{Ca}\left(\mathrm{BH}_{4}\right)_{2}$ while the $\alpha$-phase is stable in the pressure range studied, except some change in the bonding structure. Because of the poor intensity of the XRD patterns at high pressure, structure of the new phase was not determined.

Table 6. 1: Change in Raman shift frequencies as a function of pressure for B-H stretching modes of $\mathrm{Ca}\left(\mathrm{BH}_{4}\right)_{2}$ during compression and decompression.

\begin{tabular}{|c|c|c|c|c|}
\hline \multicolumn{5}{|c|}{ B-H stretching $\quad \mathrm{d} v / \mathrm{d} P\left(\mathrm{~cm}^{-1} \mathrm{GPa}^{-1}\right)$ at different $\mathrm{P}$ - ranges } \\
\hline$\left(\mathrm{cm}^{-1}\right)$ & $0.2-8.8 \mathrm{GPa}$ & $10.2-25.3 \mathrm{GPa}$ & $0.2-4.9 \mathrm{GPa}$ & $6.5-16 \mathrm{GPa}$ \\
\hline 2298 & $6.04(6)$ & - & - & $6.18(2)$ \\
\hline 2307 & -- & $8.38(7)$ & $10.81(3)$ & $10.81(3)$ \\
\hline 2330 & $8.69(1)$ & 7.99(4) & $12.9(8)$ & $12.9(8)$ \\
\hline 2375 & $6.79(5)$ & $8.92(6)$ & -- & -- \\
\hline 2423 & 9.39 (11) & -- & $5.85(11)$ & -- \\
\hline 2466 & $4.5(12)$ & -- & -- & -- \\
\hline
\end{tabular}




\section{CHAPTER 7. HIGH PRESSURE INVESTIGATION AND \\ P-T PHASE DIAGRAM OF NaBH}

\subsection{Introduction}

Sodium borohydride $\left(\mathrm{NaBH}_{4}\right)$ is a potential hydrogen storage material and has a theoretical hydrogen storage capacity of $10.6 \mathrm{wt} \%$. Li et al. [165], demonstrated $\mathrm{NaBH}_{4}$ slurry as an efficient way of application and generated hydrogen by the simple reaction $\mathrm{NaBH}_{4}+2 \mathrm{H}_{2} \mathrm{O} \rightarrow \mathrm{NaBO}_{2}+4 \mathrm{H}_{2}$. Due to strong covalent and ionic bonding nature, dissociation temperatures of borohydrides are very high. The improvement of the hydride properties by catalytic addition requires better understanding of the phases and its phase stability. It is found that the hydrogen desorption in $\mathrm{NaBH}_{4}$ can be enhanced by addition of $\mathrm{Pt}$ or $\mathrm{Ru}$ [166]. Under ambient conditions, $\mathrm{NaBH}_{4}$ has a cubic structure with space group Fm-3m [85, 167].

At low temperatures, around $190 \mathrm{~K}, \mathrm{NaBH}_{4}$ exists in a tetragonal structure [168, 169]. The well investigated high- pressure phases of $\mathrm{NaBH}_{4}$ at room temperature are tetragonal- $\mathrm{P}^{-} 42_{1} \mathrm{c}$ and orthorhombic- Pnma structure which appears above 6.3 and 8.9 GPa, respectively [85]. The previous reports suggest that the orthorhombic phase is stable in the pressure range $8.9-30 \mathrm{GPa}$. Lee et al. [170], have studied ab initio structural stability of cubic and tetragonal phases of $\mathrm{NaBH}_{4}$ up to $30 \mathrm{GPa}$ and $4000 \mathrm{~K}$ but an experimental phase diagram is lacking in the current literature in these ranges. A low temperature phase diagram of $\mathrm{NaBH}_{4}$ was reported by Sundqvist et al. [169], in the P- T plane of 0-2 GPa and 100-300 K. In the phase diagram reported by Sundqvist et al. [169], tetragonal to orthorhombic phase boundary of $\mathrm{NaBH}_{4}$ is marked in the range 9-11 GPa. 
Even though there are many reports on high pressure phase transitions of $\mathrm{NaBH}_{4}$, some of them failed to observe a phase transition above $10.8 \mathrm{GPa}$ [169]. The ab initio calculations by Araujo et al. [171], showed a cubic to monoclinic transition at $19 \mathrm{GPa}$ and to orthorhombic at $33 \mathrm{GPa}$. Through Raman spectroscopic studies, the same group observed a phase transition in the range $\sim 10.8-14.8 \mathrm{GPa}$ and a completely new phase was formed above $15 \mathrm{GPa}$. However, the XRD experiments reported cubic to tetragonal transition at $6.3 \mathrm{GPa}$ and to orthorhombic at $8.9 \mathrm{GPa}[84,85]$. Because of these inconsistencies in reported transition pressures and structure of the phases, in situ high PT structural measurements were carried out on $\mathrm{NaBH}_{4}$ both by XRD and Raman spectroscopy, using a DAC to obtain further understanding of its stability.

Filinchuk et al. [172], found that texture has much influence in the structure refinement of $\mathrm{NaBH}_{4}$. Theoretically predicted structures of borohydrides (Ref. [148, 173]) are found to be different from that of the experimentally observed ones $[80,85,94$, 171]. More theoretical and experimental investigations are required to explain the inconsistencies in these results. The present work is to extend the P-T plane of the experimental $\mathrm{NaBH}_{4}$ phase diagram from ambient to $17 \mathrm{GPa}$ and $673 \mathrm{~K}$ using combined XRD and Raman spectroscopy studies. The results of the high pressure Raman spectroscopic studies of $\mathrm{NaBH}_{4}$ up to $\sim 30 \mathrm{GPa}$ is carried out and an interesting new phase is observed. A comparative symmetry analysis also has been carried out for various phases of $\mathrm{NaBH}_{4}$. 


\subsection{Experimental Details}

$\mathrm{NaBH}_{4}$ of $99 \%$ purity, purchased from Sigma Aldrich was used for the study. All sample handlings were carried out in an argon atmosphere. Mao-Bell type DAC with diamonds of $0.4 \mathrm{~mm}$ culets and steel gasket of $0.18 \mathrm{~mm}$ hole was used. For heating experiments resistive ring-heater and specially designed $\mathrm{K}$ - type thermocouples were employed around diamond culets. For accurate pressure measurement, $\mathrm{NaCl}$ and ruby chips were added to the sample. High pressure experiments were conducted with and without mineral oil as a pressure transmitting medium and observed highly non hydrostatic behavior above $12 \mathrm{GPa}$ in both cases. In the heating experiments no pressure transmitting medium was used. The XRD measurements at high P-T conditions were conducted at station B2 of Cornell high energy synchrotron source (CHESS) in Cornell University with facilitating radiation of wavelength, $\lambda=0.49594$ Some part of the high P-T diffraction studies was carried out at X17B2 beam line of National Synchrotron Light Source in Brookhaven National Laboratory (energy- dispersive, $2 \theta=6.4762$. High pressure XRD experiment was also carried out at HPCAT, Argonne National Laboratory with an X-ray wavelength of $\lambda=0.40026 \AA$.

\subsection{Results and Discussion}

\subsubsection{In situ high P-T X-ray diffraction}

\subsubsection{Angle dispersive micro X-ray diffraction}

The micro XRD patterns of $\mathrm{NaBH}_{4}$ were refined with Rietveld analysis and the obtained lattice parameter for the ambient cubic phase is a $=6.107(0.2) \AA$ with $\mathrm{V}=$ $227.83 \AA^{3}$ at $0.2 \mathrm{GPa}$ which is in agreement with the previously reported value [85]. 
Figure 7.1 shows the indexed synchrotron XRD pattern of cubic $\mathrm{NaBH}_{4}$ with the results of Rietveld analysis. In the cubic phase, the $\mathrm{Na}$ atoms occupy $4 \mathrm{a}$ sites at $(0,0,0)$ and $\mathrm{B}$ atoms at $4 \mathrm{~b}(0.5,0.5,0.5)$ positions, whereas the $\mathrm{H}$ atoms occupy $32 \mathrm{f}$ sites with coordinates $\mathrm{x}=0.400264$. The orientationally disordered $\left(\mathrm{BH}_{4}\right)^{-}$tetrahedral units are octahedrally surrounded by $\mathrm{Na}^{+}$cations facilitating ionic nature for the compound.

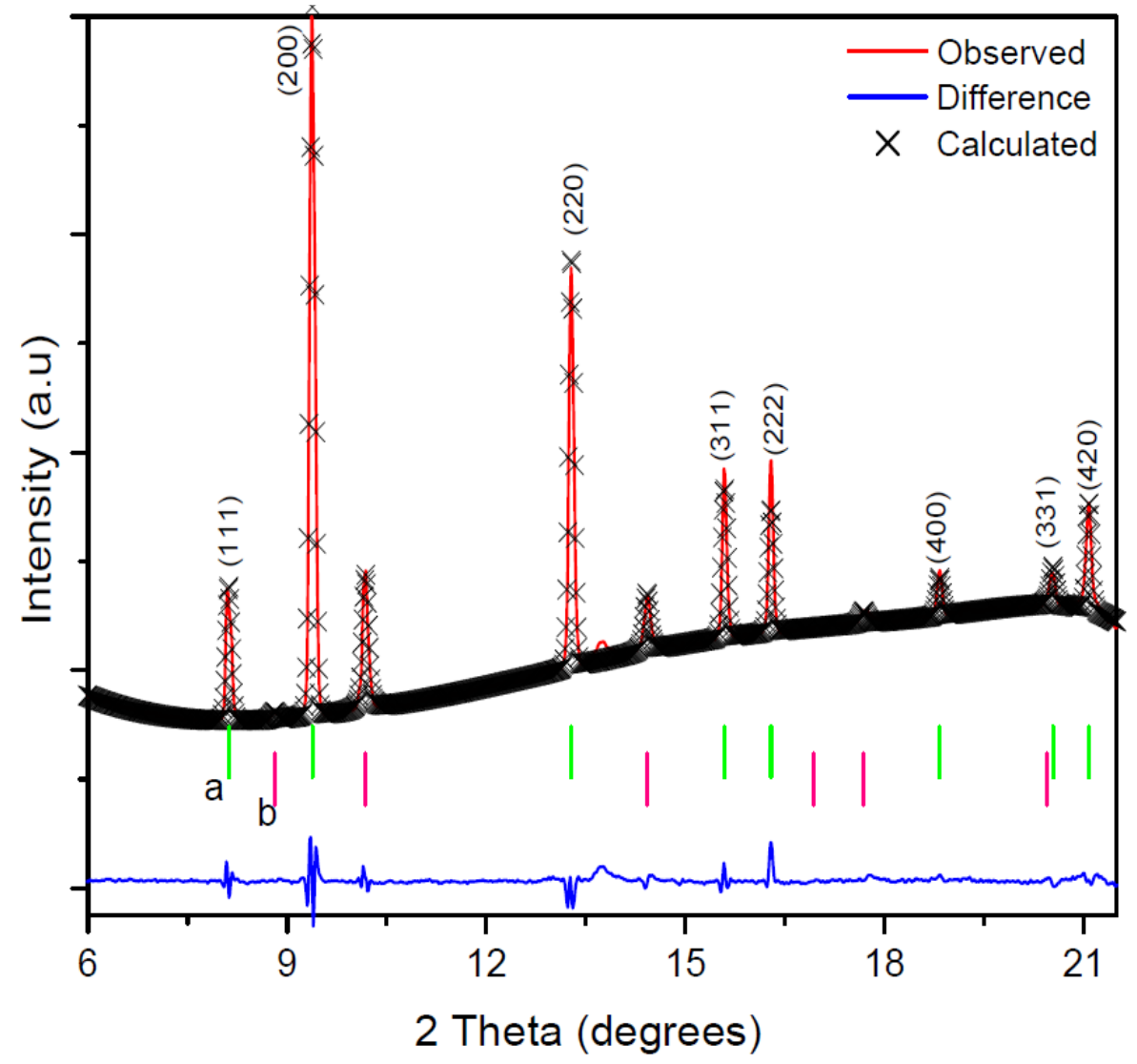

Figure 7. 1: Micro X-ray diffraction pattern of the cubic phase of $\mathrm{NaBH}_{4}$ collected at 0.8 GPa using a synchrotron source with the results of Rietveld analysis. The peak positions marked in green (a) corresponds to that of $\mathrm{NaBH}_{4}$ and that marked in pink (b) are of $\mathrm{NaCl}$. 
Figure 7.2 shows selected X-ray diffraction patterns collected at different pressures/ temperatures. The cubic structure is stable below $6.5 \mathrm{GPa}$ and further increase in pressure cause splitting of (200), (220) and (311) lines which indicate a transition to tetragonal phase. XRD pattern collected at $9 \mathrm{GPa}$ shows appearance of many new peaks which corresponds to an orthorhombic phase. The tetragonal phase at $6.5 \mathrm{GPa}$ transformed back to cubic phase when temperature was increased to $373 \mathrm{~K}$ and the compression increases this transformation temperature.

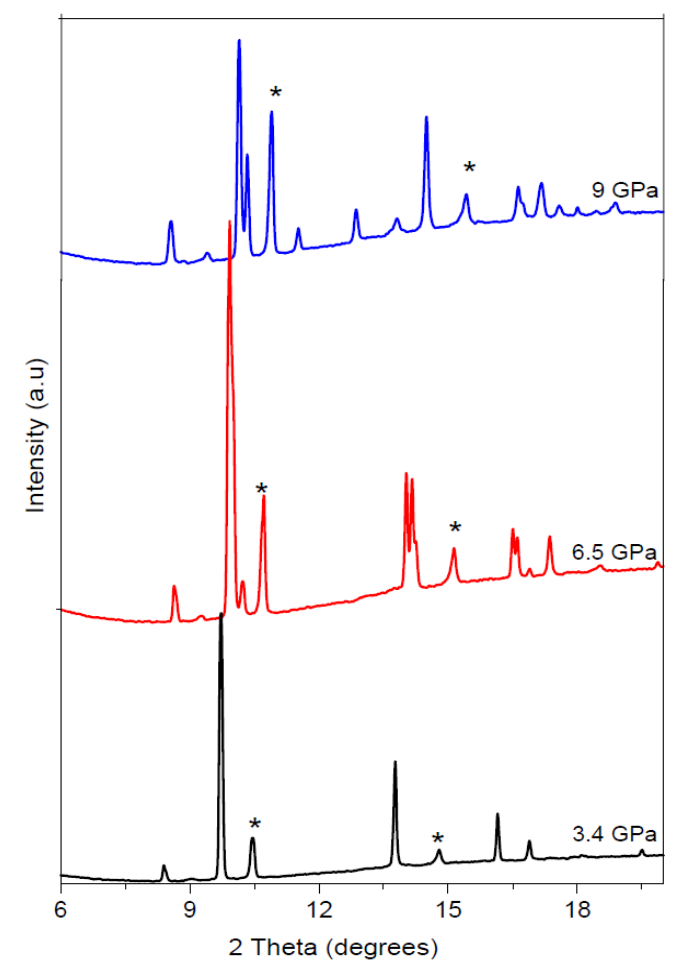

(a)

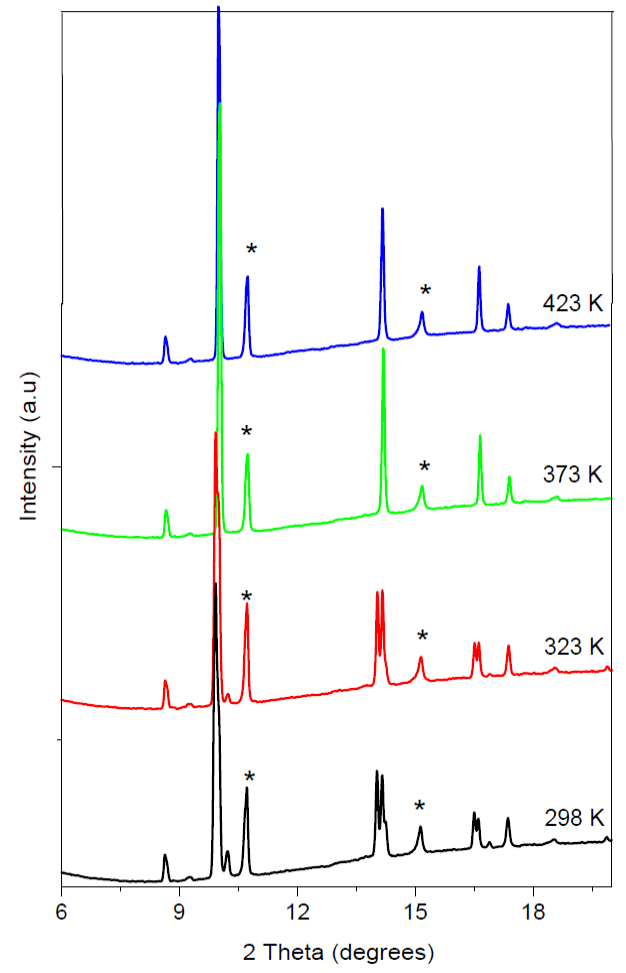

(b)

Figure 7. 2: Selected XRD patterns of the three different phases of the $\mathrm{NaBH}_{4}$, at room temperature; (a) the cubic (3.4 GPa), tetragonal (6.5 GPa) and orthorhombic (9 GPa). (b) at various temperatures and $6.5 \mathrm{GPa}$. Between 323 and $373 \mathrm{~K}$, tetragonal to cubic phase transition occurs. Peaks marked ${ }^{*}$ are of $\mathrm{NaCl}$ which was used as a pressure marker. 
Figure 7.3 shows schematic representation of the crystal structures of three different phases of $\mathrm{NaBH}_{4}$. The high pressure XRD experiments were conducted up to 34 GPa to investigate a possible phase transition above $14 \mathrm{GPa}$ which was observed with Raman spectroscopy (discussed later). Figure 7.4 shows XRD patterns collected above 13 GPa for some selected pressures. Even though a new peak observed above $15.7 \mathrm{GPa}$ (marked by arrow in successive patterns), a phase transition is not clearly visible from the peak revolution. This is due highly non hydrostatic pressure and the broadening of peaks above $12 \mathrm{GPa}$. The experiments carried out with and without mineral oil as a pressure medium. Due to solidification of the mineral oil above 9 GPa non hydrostaticity is generated. All the phase transitions observed were reversible as can be inferred from the XRD pattern of decompressed sample.

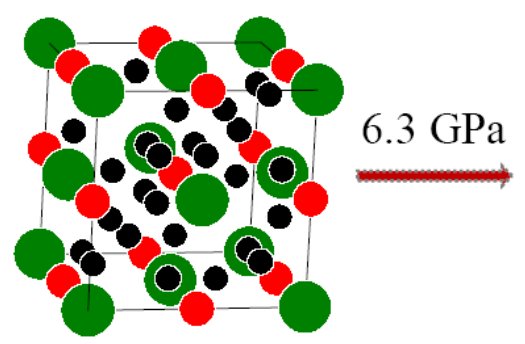

(a)

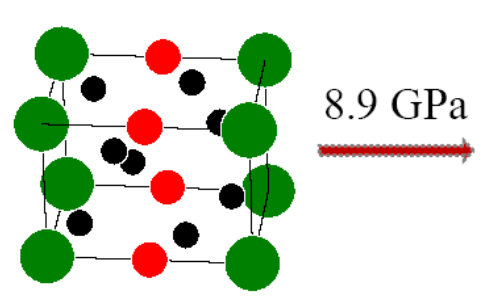

(b)

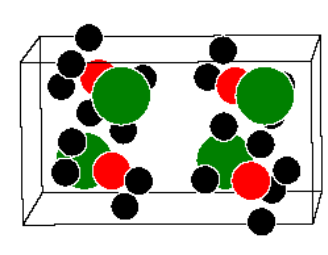

(c)

Figure 7. 3: Crystal structures of (a) the cubic, (b) tetragonal and (c) orthorhombic phases of $\mathrm{NaBH}_{4}$. The $\mathrm{Na}, \mathrm{B}$, and $\mathrm{H}$ are marked in green, orange and black symbols, respectively.

In the cubic- $\mathrm{NaBH}_{4}$, various bond lengths are found to be 1.208- 1.709, 1.047, 3.03 and $2.57 \AA$ for $\mathrm{H}-\mathrm{H}, \mathrm{B}-\mathrm{H}, \mathrm{Na}-\mathrm{B}$ and Na-H, respectively at $0.8 \mathrm{GPa}$. Except $\mathrm{Na}-\mathrm{H}$ 
bonds all the other bonds are highly compressive. On increasing pressure from 0.8 - 3.8 GPa, Na-H and B-H bonds exhibit $1.45 \%$ and $22 \%$ compression, respectively. Hence it may expect that the distortion in $\left(\mathrm{BH}_{4}\right)^{-}$tetrahedra can be causing the phase transitions. The $\mathrm{H}-\mathrm{B}-\mathrm{H}$ and $\mathrm{B}-\mathrm{H}-\mathrm{H}$ bond angles remain unchanged in this pressure range while Na$\mathrm{H}-\mathrm{B}$ and $\mathrm{H}-\mathrm{Na}-\mathrm{H}$ angles diverge or converge. The co-ordination number and geometry of co-ordination polyhedra vary with pressure and temperature leading to phase transition.

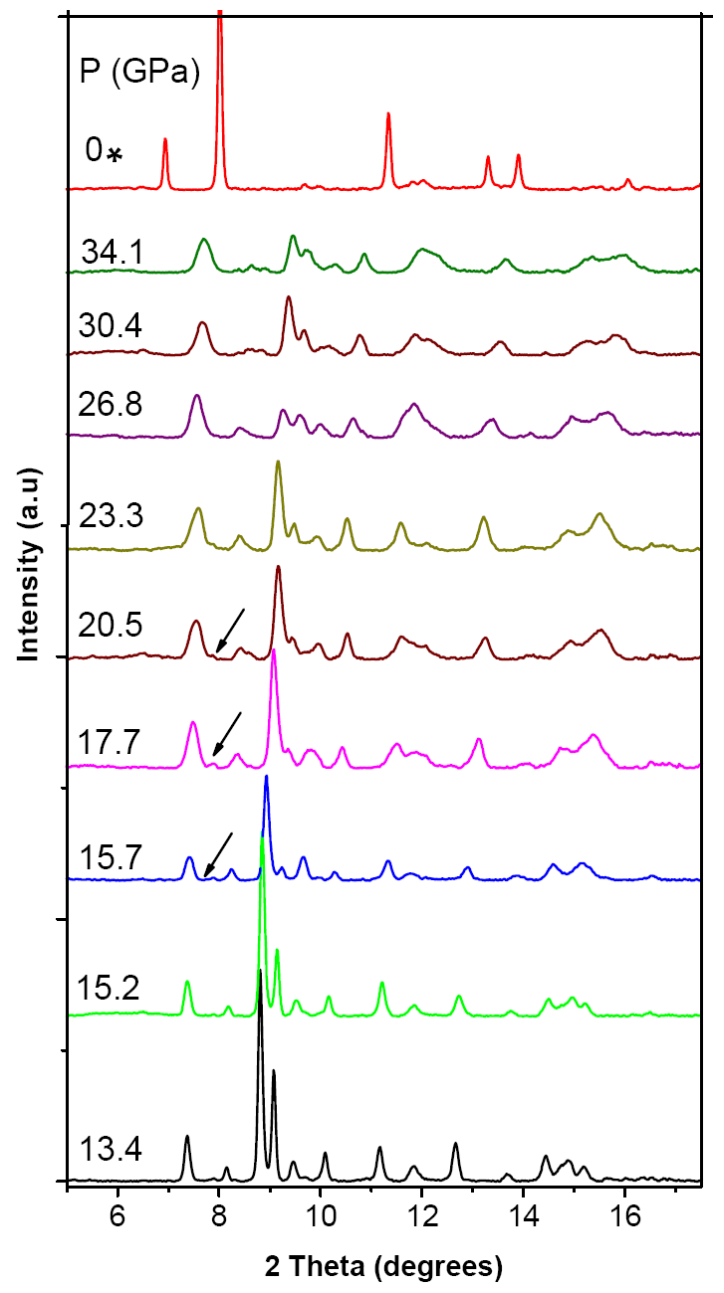

Figure 7. 4: Selected high pressure XRD patterns of $\mathrm{NaBH}_{4}$ above $13.4 \mathrm{GPa}$ up to 34 GPa. The arrow indicated new peaks, and decompressed pattern is marked *. 


\subsubsection{Energy dispersive micro X-ray diffraction}

A portion of the high P-T experiments on $\mathrm{NaBH}_{4}$ were carried out using multi anvil press employed with a cylindrical graphite heater and tungsten carbide (WC) anvils and the diffraction data were recorded using a CCD detector. Figure 7.5 shows an energy dispersive XRD (EDXRD) pattern of the cubic phase. $\mathrm{NaCl}$ was used a pressure marker. At each pressure sample was heated up to $675 \mathrm{~K}$.

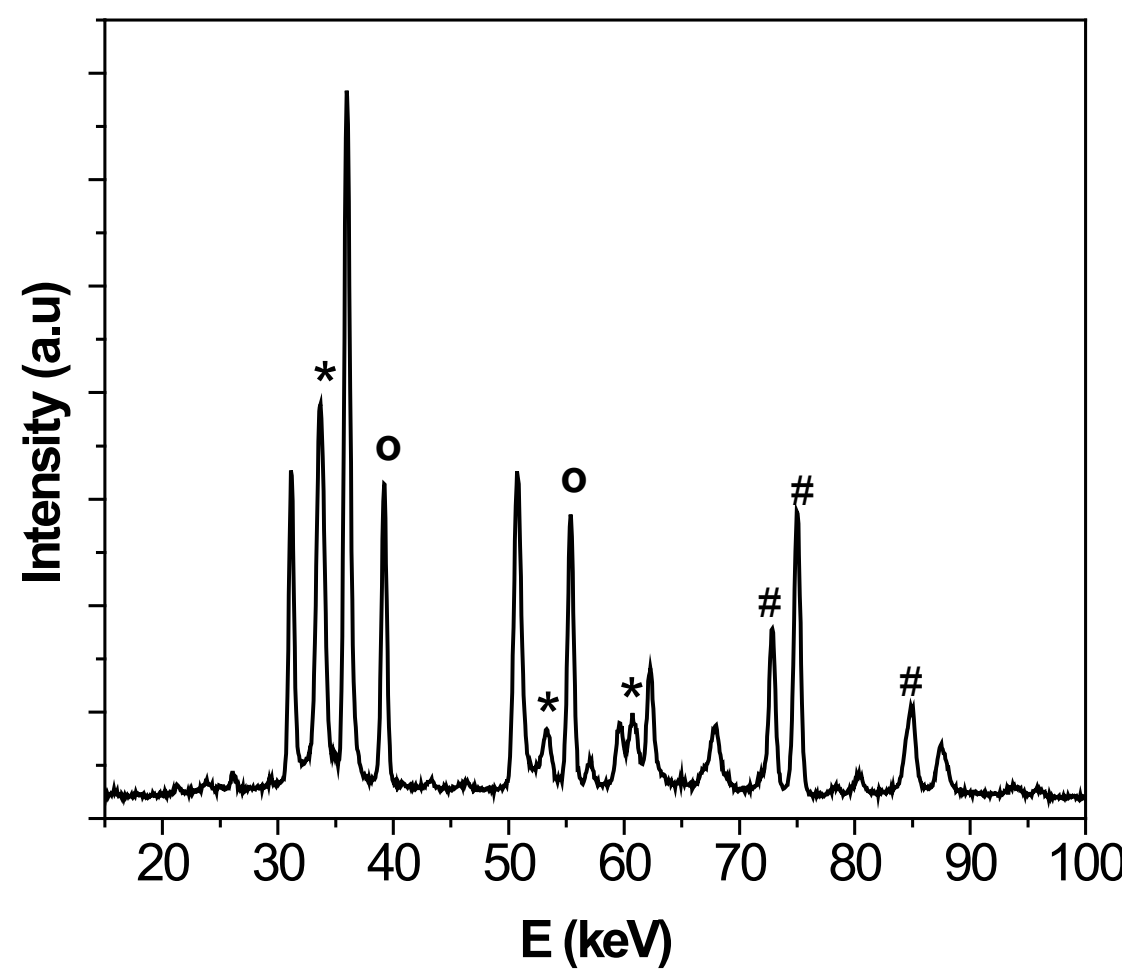

Figure 7. 5: An EDXRD pattern of cubic- $\mathrm{NaBH}_{4}$ at $0.2 \mathrm{GPa}$. The peaks marked (*), (o) and (\#) are due to $\mathrm{BN}, \mathrm{NaCl}$ and $\mathrm{K}_{\alpha}$, respectively.

The pressure- volume- temperature ( $\mathrm{P}-\mathrm{V}-\mathrm{T})$ data obtained for the cubic phase of $\mathrm{NaBH}_{4}$ was fitted with the third order Birch-Murnaghan equation of state and the value of $\mathrm{K}_{0}$ is found to be $18.76(1) \mathrm{GPa}$ with $\mathrm{K}_{\mathrm{o}}{ }^{\prime}=3.48(0.3)$ and equilibrium unit cell volume of 
231.817(0.9) $\AA^{3}$. The temperature derivative of bulk modulus and the thermal expansion coefficient were also determined for the ambient phase and the obtained values are $\mathrm{dK} / \mathrm{dT}$ $=-0.01307 \mathrm{GPaK}^{-1}$ and $\alpha=12.5 \times 10^{-5}+23.21 \times 10^{-8} \mathrm{~T} / \mathrm{K}$, respectively. Figure 7.6 show a comparison of P- V data at 298 and $573 \mathrm{~K}$. At low pressures, below $2 \mathrm{GPa}$, the data shows slight deviation from the previously reported data at room temperature by Kumar et al. [85], which can be due to an error in pressure determination due to a buildup of anisotropic stress [174].

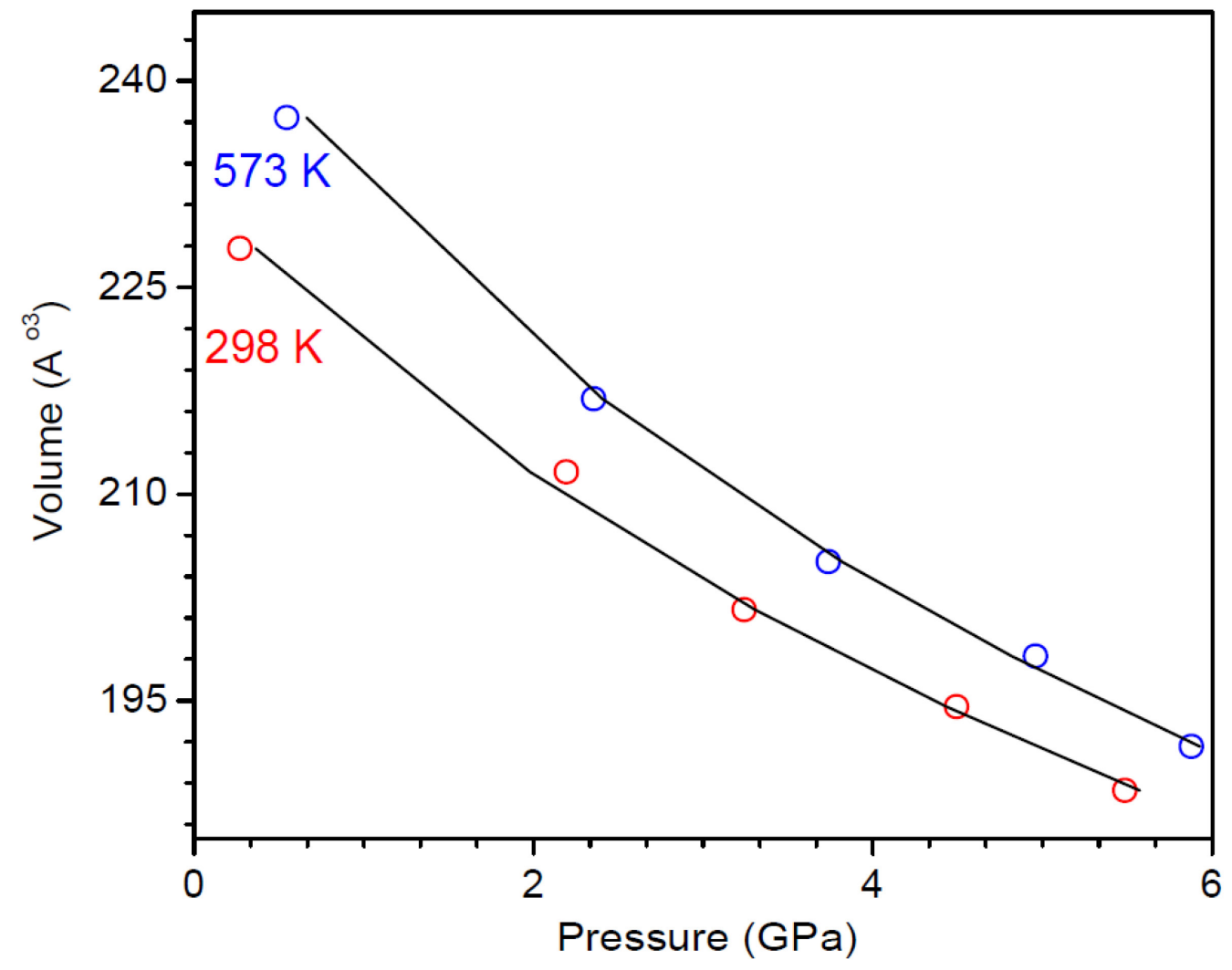

Figure 7. 6: Pressure dependence of the unit cell volume (circles) for the high-pressure phase of cubic $\mathrm{NaBH}_{4}$ during compression at room temperature and at $573 \mathrm{~K}$, fitted with third order Birch- Murnaghan EoS (line). 
The obtained value of $\mathrm{K}_{\mathrm{o}}$ for cubic phase is in agreement with the19.9 GPa reported by Kumar et al. [85]. They have also reported that the bulk modulus of orthorhombic- $\mathrm{NaBH}_{4}$ is $31.1 \mathrm{GPa}$. The ambient cubic phase of $\mathrm{NaH}$ and its high pressure phase has a bulkmodulus of 19.4 and $28.3 \mathrm{GPa}$ respectively [48]. It suggests that the boron addition to $\mathrm{NaH}$ does not have much influence on its compressibility. The calculated values of $\mathrm{K}_{0}$ for cubic and tetragonal phases of $\mathrm{NaBH}_{4}$ are 20.6 and $30.9 \mathrm{GPa}$, respectively at $0 \mathrm{~K}$ [84]. First principle calculations by Vajeeston et al. [91], shows a $\mathrm{K}_{0}$ of 7.6 GPa for the $\mathrm{NaBH}_{4}$ which is much lower than all other reported values.

\subsubsection{Raman spectroscopy}

\subsubsection{High pressure in situ Raman spectroscopy}

Figure 7.7 shows Raman spectra of $\mathrm{NaBH}_{4}$ at some selected pressures and temperatures in the B-H stretching region $\left(2100-2500 \mathrm{~cm}^{-1}\right)$. B-H bending modes (1100$1300 \mathrm{~cm}^{-1}$ ) overlap with the Raman peaks due to diamond in DAC and hence were excluded them from further analysis. According to the Raman spectroscopy results, phase transitions occur at 6.3 and $8.3 \mathrm{GPa}$ to tetragonal and orthorhombic structures respectively. No new Raman peaks emerged in the cubic to tetragonal transition except change of slope of the vibrational modes. There is a new peak appearing at $8.3 \mathrm{GPa}$ indicating tetragonal to orthorhombic phase transition. The cubic- tetragonal transition is an order-disorder transition which was also observed below $190 \mathrm{~K}$ [174]. Again a new phase starts to appear above $14 \mathrm{GPa}$ and form a completely new phase around $17 \mathrm{GPa}$ and it is stable up to $30 \mathrm{GPa}$ (Figure 7. 7b). It may be possible that the new phase is not distinguishable in XRD patters, as above $14 \mathrm{GPa}$ the peaks are much broader and weak 
which makes it difficult to identify any splitting of peaks. Above $14 \mathrm{GPa}$ the relative intensities of the peaks changes to that of a monoclinic- $\mathrm{LiAlH}_{4}$ with space group $\mathrm{P} 2{ }_{1} / \mathrm{c}$ [69]. Any anomalies in Raman shift were not observed below $3 \mathrm{GPa}$ as reported in Araujo et al. [171]

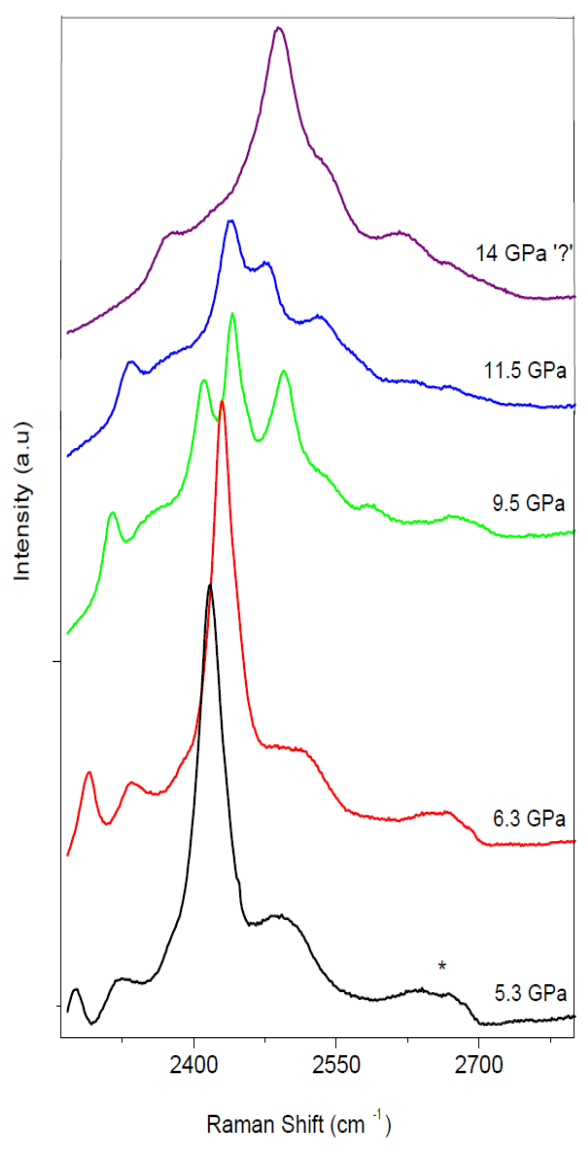

(a)

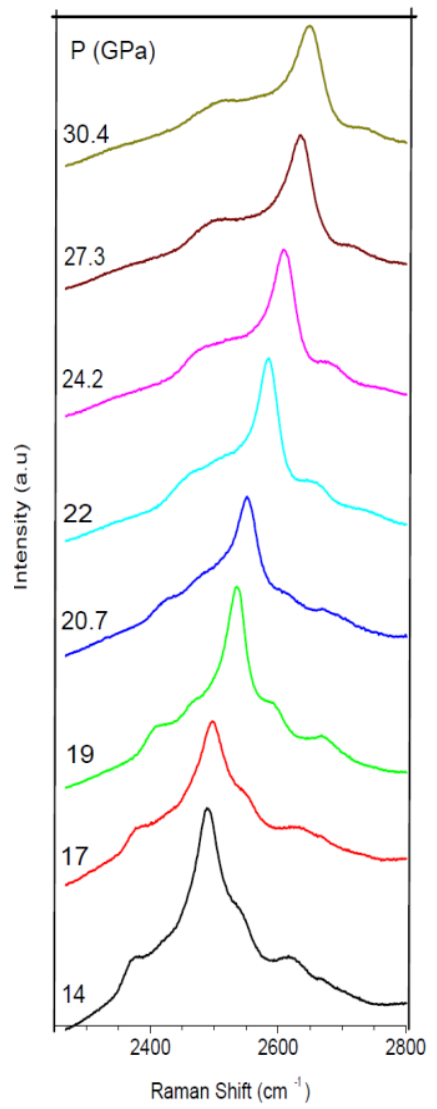

(b)

Figure 7. 7: Raman spectra of $\mathrm{NaBH}_{4}$ during compression; (a) at selected pressures and the spectrum marked ? corresponds to the appearance of a new phase, (b) spectra in the range 14-30 GPa. 
Figure 7.8 shows Raman spectra collected at various pressures during decompression. A large hysteresis $(\sim 3 \mathrm{GPa})$ is observed in the reverse transition of orthorhombic phase at room temperature. The experimentally observed B-H stretching mode frequencies for all the four phases of $\mathrm{NaBH}_{4}$ are presented in Table 7.1. The Raman mode present at $754 \mathrm{~cm}^{-1}$ in the spectra of cubic phase was not present in that of the high pressure phases. The B-H bending mode $\sim 1278 \mathrm{~cm}^{-1}$ at high pressures were not studied with pressure because of its overlap with the Raman peak of diamond from DAC.

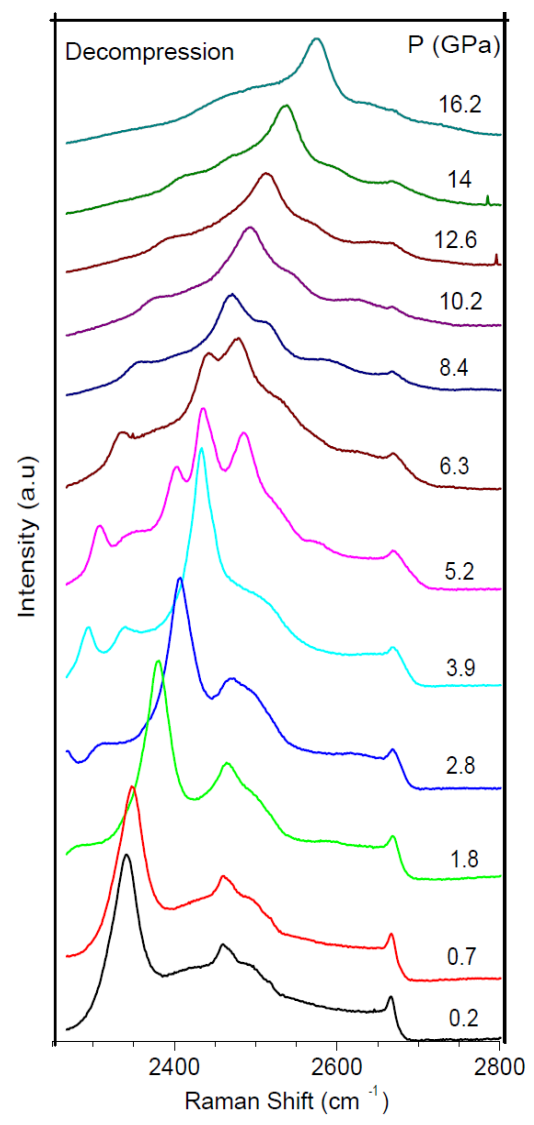

Figure 7. 8: Selected Raman spectra of $\mathrm{NaBH}_{4}$ during decompression.

The decompression data shows the phase transitions occurred are reversible with a hysteresis which supports the observation of Sundqvist et al. [169]. The observed 
transitions are much clear from the plot of Raman shift with pressure (Figure 7.9). It is found that pressure induced shift in Raman peaks arises from the bond compression and the energy storage exerted by compressive stress [175]. Frequency of Raman modes increase or stiffen the vibration with compression of bond under pressure whereas the bond expansion softens the vibration. There is a correlation between B- $\mathrm{H}$ stretching frequencies, B-H bond length and energy.

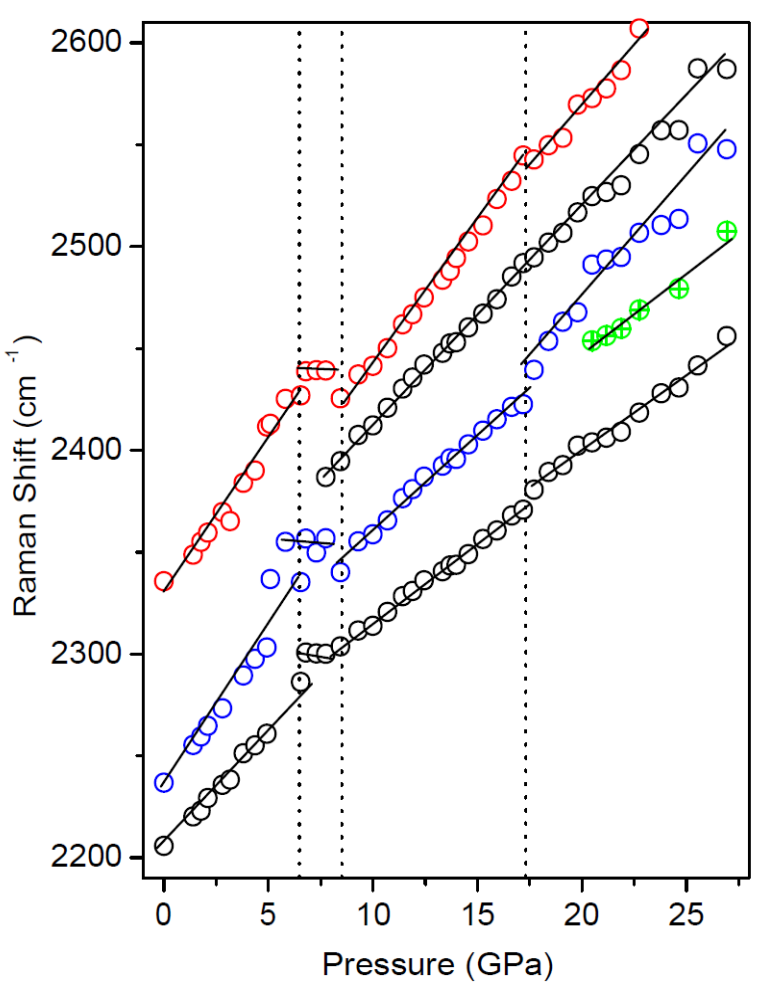

(a)

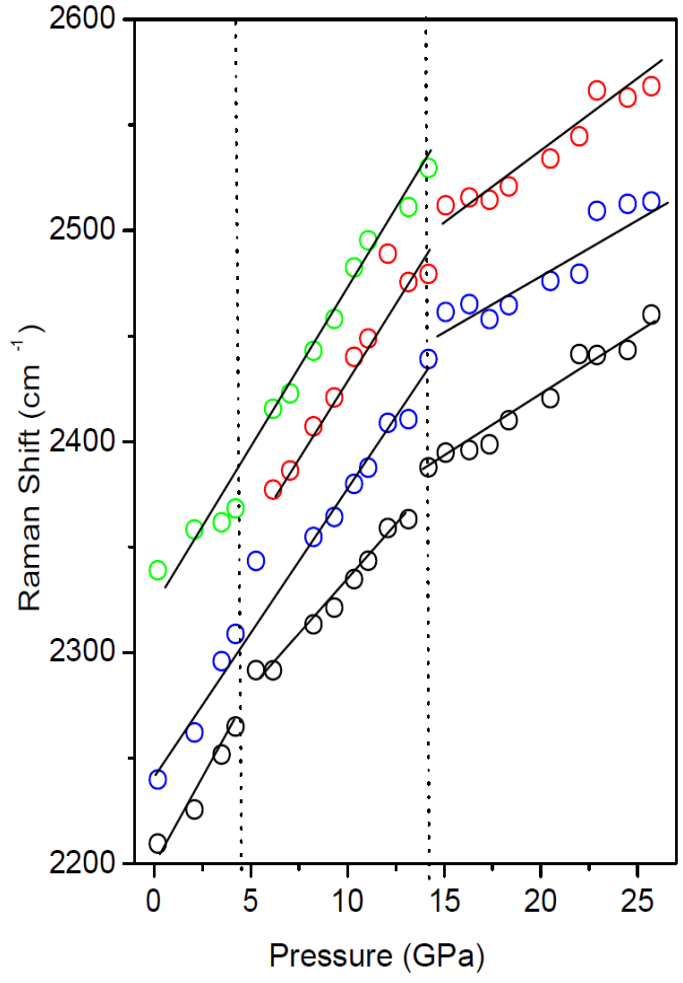

(b)

Figure 7. 9: The Raman shift versus pressure obtained by peak fitting of B-H stretching modes at each pressure; (a) during compression and (b) decompression. Pressures corresponds to phase transition are marked with grid lines parallel to the $\mathrm{Y}$ axis. 
In the tetragonal phase the stiffening of bond due to compression is absent where as all other phases has shift in Raman mode frequency. This can be related to the bond reordering under pressure accompanied with the transition of an orientationally disordered cubic to an orientationally ordered tetragonal phase. For a hydrogen bonded B-H stretching mode, the positive pressure dependence may be explained by $\mathrm{H}-\mathrm{H}$ repulsion under compression [176]. A change in the slope of Raman shift versus pressure plots and an appearance of a new peak during compression confirms the transition of orthorhombic to another high pressure phase in the range 14-17 GPa.

It is difficult to distinguish an orthorhombic to tetragonal transition from the decompression data which can be due to the large hysteresis of the reverse transition of orthorhombic phase. Table 7.2 gives the change of slopes of Raman shift versus pressure during compression which indicates that there are four high pressure phases of $\mathrm{NaBH}_{4}$ that exist in the range $0-30 \mathrm{GPa}$ at room temperature. First principle calculations by Araujo et al. [171], found a cubic to monoclinic - P2 $2_{1} / \mathrm{c}$ phase transition at $19 \mathrm{GPa}$ while their experimental Raman spectra $\sim 14 \mathrm{GPa}$ is very similar to that of the orthorhombic phase as observed in this study.

Calculations of Kim et al. [84], and experiments of Kumar et al. [85], found that orthorhombic phase is stable up to $30 \mathrm{GPa}$. The compression data of Araujo et al. [171], shows a complete transition to an orthorhombic phase $\sim 14.8 \mathrm{GPa}$ which reverse transform to low pressure phase at $7 \mathrm{GPa}$ on decompression. This result deviates from the observations of transition pressures of 8.3 and $5 \mathrm{GPa}$ for forward and reverse transitions of orthorhombic phase, respectively in this study. The difference in the measured 
pressures might be the reason why they have missed the phase transition above $14 \mathrm{GPa}$ in their Raman spectroscopic study up to $16.2 \mathrm{GPa}$.

Table 7. 1: The observed B-H stretching modes in $\mathrm{cm}^{-1}$ (sh- shoulder) for different phases of $\mathrm{NaBH}_{4}$.

\begin{tabular}{lllllll}
\hline $\begin{array}{l}\text { OGPa } \\
\text { cubic }\end{array}$ & $\begin{array}{l}\text { 6.3GPa } \\
\text { tetragonal }\end{array}$ & & $\begin{array}{l}\text { 9.1GPa } \\
\text { orthorhombic }\end{array}$ & \multicolumn{3}{l}{$\begin{array}{l}\text { 17.2GPa } \\
\text { monoclinic }\end{array}$} \\
\hline 2205.77 & 2286.62 & & 2311.57 & & 2368.12 & sh \\
2236.77 & 2335.59 & sh & 2356.74 & sh & 2408.61 & sh \\
2331.7 sh & 2384.34 & sh & 2407.54 & & -- & \\
-- & -- & & 2436.89 & & 2452.84 & sh \\
2335.66 & 2426.13 & sh & 2450.44 & sh & -- & \\
2350.73 & 2430.05 & & 2475.74 & sh & 2491.05 & \\
-- & -- & & 2492.85 & & 2534.12 & sh \\
\hline 2461.91 sh & 2512.4 & & 2533.68 & sh & 2613.65 & sh \\
-- & -- & & 2591.52 & sh & 2671.24 & sh \\
\hline
\end{tabular}

Table 7. 2: The pressure dependence of Raman shift frequency for $\mathrm{NaBH}_{4}$ during both compression and decompression.

\begin{tabular}{|c|c|c|c|c|c|}
\hline \multicolumn{2}{|c|}{$\begin{array}{l}\text { Raman mode } \\
v\left(\mathrm{~cm}^{-1}\right)\end{array}$} & \multicolumn{2}{|c|}{$\begin{array}{c}\text { Compression } \\
\mathrm{dv} / \mathrm{dp}\left(\mathrm{cm}^{-1} \mathrm{GPa}^{-1}\right)\end{array}$} & \multicolumn{2}{|c|}{$\begin{array}{l}\text { Decompression } \\
\mathrm{dv} / \mathrm{dp}\left(\mathrm{cm}^{-1} \mathrm{GPa}^{-1}\right)\end{array}$} \\
\hline & $0-6.3 \mathrm{GPa}$ & 8.3-17 GPa & $17.5-28 \mathrm{GPa}$ & $5.2-13.6 \mathrm{GPa}$ & $14.2-26 \mathrm{GPa}$ \\
\hline 2200 & $11.51(1)$ & $8.2(7)$ & $7.56(3)$ & $9.9(0.5)$ & $6.23(0.4)$ \\
\hline 2300 & $13.82(2)$ & $8.88(2)$ & $11.45(8)$ & $12.97(0.8)$ & $6.05(0.7)$ \\
\hline 2340 & $15.93(1)$ & $13.32(3)$ & 11.97(9) & $14.66(0.6)$ & -- \\
\hline 2400 & -- & $10.39(1)$ & -- & $16.04(2)$ & $6.07(0.6)$ \\
\hline
\end{tabular}

\subsubsection{High P-T Raman spectroscopy}

High P-T Raman spectroscopic studies were carried out to construct the phase diagram of $\mathrm{NaBH}_{4}$. Pressure was measured at room temperature and then heated the sample in steps. The high pressure phase transitions were reversible with a rise in 
temperature as can be observed from Figures 7.10. After collecting spectra at each temperature until the phase transition, the DAC was cooled down to room temperature to increase the pressure with ruby fluorescence method. The transition temperatures were noted on both heating and cooling cycles and the average value is used as a transition point in the phase diagram.

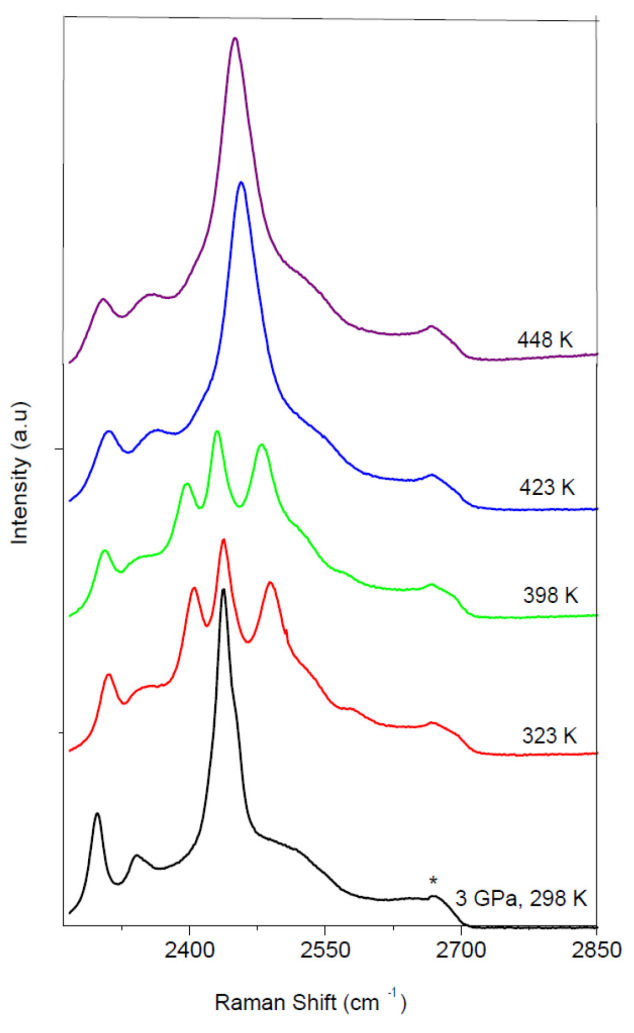

(a)

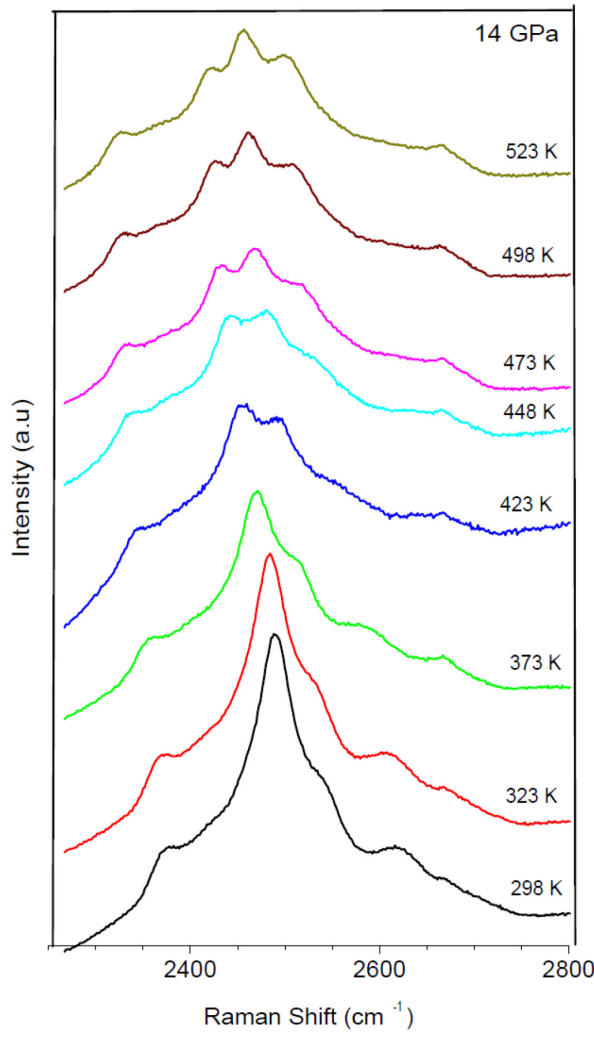

(b)

Figure 7. 10: The high temperature Raman spectra of $\mathrm{NaBH}_{4}$ in the B-H stretching mode region; (a) while heating at $9.5 \mathrm{GPa}$ in comparison with a spectrum at $3 \mathrm{GPa}$, (b) at 14 GPa and different temperatures. 


\subsubsection{P-T phase diagram}

The transition points for $\mathrm{NaBH}_{4}$ at various pressures and temperature were noted from both XRD and Raman spectroscopy experiments and constructed a P- T phase diagram. Figure 7.11 shows phase diagram of the $\mathrm{NaBH}_{4}$ in the pressure range $0-17 \mathrm{GPa}$ and temperature range $175-673 \mathrm{~K}$. The phase boundaries are obtained by heating at almost constant pressure. The cubic phase is found to be stable up to $673 \mathrm{~K}$ and $6 \mathrm{GPa}$. On increasing pressure at room temperature, the cubic- $\mathrm{NaBH}_{4}$ transform to tetragonal phase at $6.5 \mathrm{GPa}$ and back transform to cubic at $373 \mathrm{~K}$.

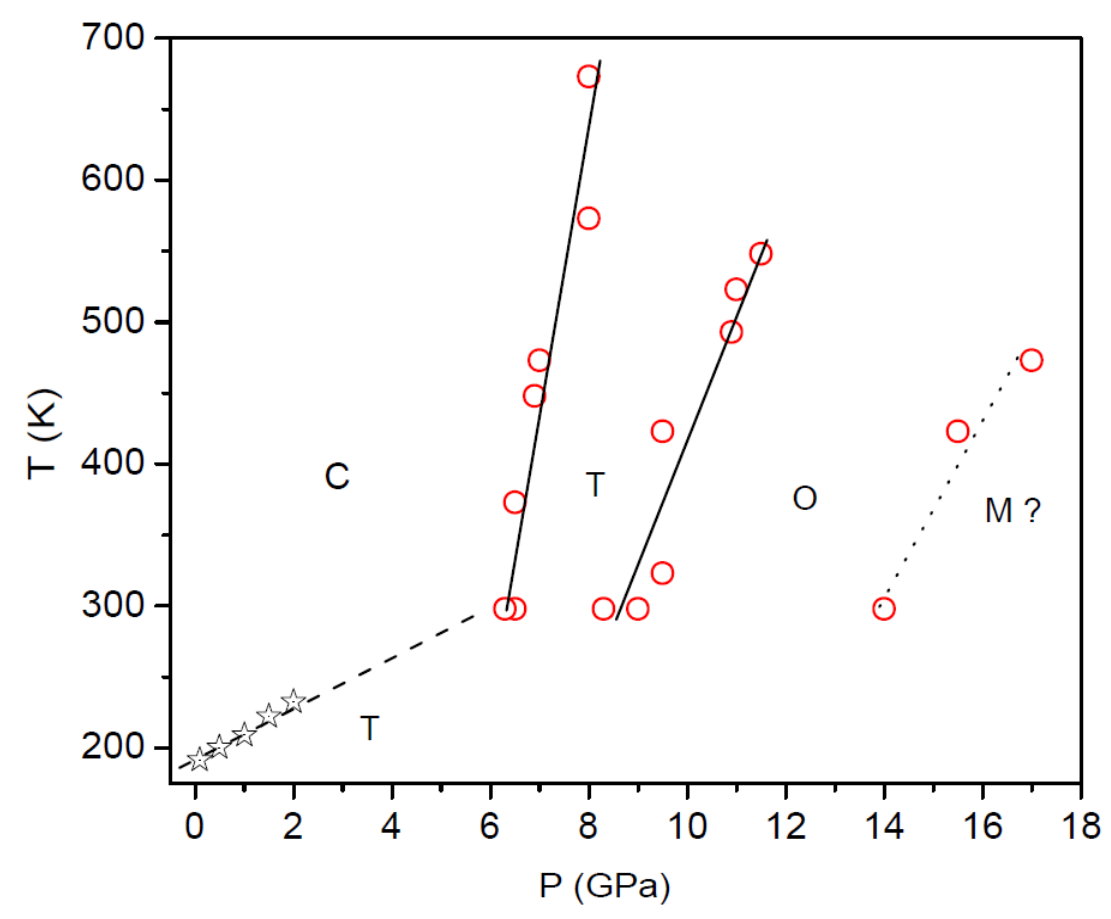

Figure 7. 11: $\mathrm{P}-\mathrm{T}$ phase diagram of $\mathrm{NaBH}_{4}$ investigated through $\mathrm{DAC}$ technique using XRD and Raman spectroscopic measurements (circles) including the results of low temperature study of Sundqvist et al. [169], (asterisks). The phase regions marked C, T, $\mathrm{O}$ and $\mathrm{M}$ are cubic, tetragonal, orthorhombic and monoclinic, respectively. The region marked ? implies structure of phase is not confirmed. 
The phase transitions observed in $\mathrm{NaBH}_{4}$ are found to be reversible with increase in temperature or decrease in pressure with some hysteresis. The available low temperature P-T data were incorporated from Sundqvist et al. [169], in the current phase diagram which is compatible with the present results. The cubic to tetragonal structural transition has a narrow phase boundary compared to that of tetragonal to orthorhombic transition implies entropy of cubic phase is comparable to that of the tetragonal phase. A large slope observed for the cubic - tetragonal phase boundary thus can be correlated to a significant volume change according to the Clapeyron equation, $\mathrm{dT} / \mathrm{dP}=\Delta \mathrm{V} / \Delta \mathrm{S}$. The molar volumes of cubic, tetragonal and orthorhombic phases are found to be 34.3, 28.14 and $26.11 \mathrm{~cm}^{3} / \mathrm{mol}$, respectively which suggests a larger slope for the cubic- tetragonal than the tetragonal- orthorhombic phase boundary.

\subsubsection{Correlation of site group to factor group and Raman activity}

To identify the Raman active vibrational modes of different phases of $\mathrm{NaBH}_{4}$, a factor group analysis also have been carried out $[177,178]$. The lattice modes of each site and the total irreducible representations are summarized in Table 7.3. Total irreducible representation of cubic $\left(\mathrm{Oh}^{5}\right)$ phase of $\mathrm{NaBH}_{4}$ is found to be $\Gamma_{\text {tot }}=\mathrm{A}_{1 \mathrm{~g}}+\mathrm{A}_{2 \mathrm{u}}+\mathrm{E}_{\mathrm{u}}+\mathrm{E}_{\mathrm{g}}+$ $\mathrm{T}_{2 \mathrm{u}}+2 \mathrm{~T}_{2 \mathrm{~g}}+4 \mathrm{~T}_{1 \mathrm{u}}+\mathrm{T}_{1 \mathrm{~g}}$ which suggest that it has 30 degrees of vibrational freedom among which $\mathrm{T}_{1 \mathrm{u}}$ is an acoustical mode and $\mathrm{A}_{1 \mathrm{~g}}, \mathrm{E}_{\mathrm{g}}$ and $\mathrm{T}_{2 \mathrm{~g}}$ are Raman active modes. Therefore there should be 4 fundamental vibrational modes in the Raman spectra of cubic phase.

Tetragonal $\left(\mathrm{D}_{2 \mathrm{~d}}{ }^{4}\right)$ phase with $\Gamma_{\text {tot }}=3 \mathrm{~A}_{1}+3 \mathrm{~A}_{2}+5 \mathrm{~B}_{1}+5 \mathrm{~B}_{2}+10 \mathrm{E}$ has 36 degrees of vibrational freedom among which $\mathrm{B}_{2}$ and $\mathrm{E}$ are acoustical modes and $\mathrm{A}_{1}, \mathrm{~B}_{1}, \mathrm{~B}_{2}$ and $\mathrm{E}$ are Raman active modes. This implies there should be 21 fundamental vibrational modes in 
the Raman spectra of tetragonal phase. Further the orthorhombic phase of $\left(\mathrm{D}_{2 \mathrm{~h}}{ }^{16}\right) \mathrm{NaBH}_{4}$ has $\Gamma_{\text {tot }}=9 A_{g}+6 A_{u}+6 B_{1 g}+9 B_{1 u}+9 B_{2 g}+6 B_{2 u}+6 B_{3 g}+9 B_{3 u}$ and 60 degrees of vibrational freedom among which $\mathrm{B}_{1 \mathrm{u}}, \mathrm{B}_{2 \mathrm{u}}$ and $\mathrm{B}_{3 \mathrm{u}}$ are acoustical modes and $\mathrm{A}_{\mathrm{g}}, \mathrm{B}_{1 \mathrm{~g}}, \mathrm{~B}_{2 \mathrm{~g}}$ and $\mathrm{B}_{3 \mathrm{~g}}$ are Raman active modes. It gives 30 fundamental vibrational modes in the Raman spectra of orthorhombic phase. A monoclinic- $\mathrm{P} 2{ }_{1} / \mathrm{c}$ structure $\left(\mathrm{C}_{2 \mathrm{~h}}{ }^{5}\right)$ can be assigned to the $\mathrm{NaBH}_{4}$ phase appeared above $14 \mathrm{GPa}$ in comparison to the theoretical prediction of Araujo et al. [171]. The monoclinic phase has 24 degrees of vibrational freedom $\left(\Gamma_{\text {tot }}=3 \mathrm{~A}_{\mathrm{g}}+9 \mathrm{~A}_{\mathrm{u}}+\right.$ $3 \mathrm{~B}_{\mathrm{g}}+9 \mathrm{~B}_{\mathrm{u}}$ ) and among which $\mathrm{A}_{\mathrm{u}}$ and $\mathrm{B}_{\mathrm{u}}$ are acoustical modes and $\mathrm{A}_{\mathrm{g}}$ and $\mathrm{B}_{\mathrm{g}}$ are Raman active modes. Therefore there should be 6 fundamental vibrational modes in the Raman spectra of monoclinic phase.

The observed Raman spectra are in agreement with the results of the factor group analysis which shows an increment in the degrees of vibrational freedom and the number of Raman active modes with pressure, from cubic to tetragonal and to orthorhombic phase transitions and then a drop at the formation of the new phase. Therefore, it can be concluded that the high pressure phase observed above $14 \mathrm{GPa}$ can be of monoclinic structure with space group $\mathrm{P} 2{ }_{1} / \mathrm{c}$ as showed by calculations of Araujo et al. [171]. The theoretically predicted monoclinic phase could be observed through Raman spectroscopy unlike in XRD because of its ability to distinguish small traces of various local phases coexisting in a compound [179]. 
Table 7. 3: Symmetry analysis and irreducible representation of lattice modes of $\mathrm{NaBH}_{4}$.

\begin{tabular}{|c|c|c|}
\hline Phase & $\begin{array}{l}\text { Translational modes of } \\
\text { atoms in each site }\end{array}$ & Total irreducible representation \\
\hline \multicolumn{2}{|c|}{ Cubic $\left(225 ; \mathrm{Fm}^{-} 3 \mathrm{~m} ; \mathrm{Oh}^{5}\right)$} & $\begin{array}{c}\Gamma_{\text {tot }}=A_{1 g}+A_{2 u}+E_{u}+E_{g}+T_{2 u}+ \\
2 T_{2 g}+4 T_{1 u}+T_{1 g}\end{array}$ \\
\hline $\mathrm{Na}(4 \mathrm{a})$ & $\mathrm{O}_{\mathrm{h}}: \mathrm{T}_{1 \mathrm{u}}$ & \\
\hline B (4b) & $\mathrm{O}_{\mathrm{h}}: \mathrm{T}_{1 \mathrm{u}}$ & \\
\hline $\mathrm{H}$ (32f) & $\mathrm{C}_{3 \mathrm{v}}: \mathrm{A}_{1 \mathrm{~g}}+\mathrm{A}_{2 \mathrm{u}}+\mathrm{E}_{\mathrm{u}}+\mathrm{E}_{\mathrm{g}}+\mathrm{T}_{2 \mathrm{u}}$ & $2 \mathrm{~T}_{1 \mathrm{u}}+\mathrm{T}_{1 \mathrm{~g}}$ \\
\hline \multicolumn{3}{|c|}{ Tetragonal $\left(114 ; \mathrm{P}-42{ }_{1} \mathrm{c} ; \mathrm{D}_{2 \mathrm{~d}}{ }^{4}\right)$} \\
\hline $\mathrm{Na}(2 \mathrm{a})$ & $\mathrm{S}_{4}: \mathrm{B}_{1}+\mathrm{B}_{2}+2 \mathrm{E}$ & \\
\hline B (2b) & $\mathrm{S}_{4}: \mathrm{B}_{1}+\mathrm{B}_{2}+2 \mathrm{E}$ & \\
\hline $\mathrm{H} \quad(8 \mathrm{e})$ & $\mathrm{C}_{1}: 3 \mathrm{~A}_{1}+3 \mathrm{~A}_{2}+3 \mathrm{~B}_{1}+3 \mathrm{~B}_{2}+6$ & \\
\hline \multicolumn{2}{|c|}{ Orthorhombic (62; Pnma; $\left.\mathrm{D}_{2 \mathrm{~h}}{ }^{16}\right)$} & $\begin{array}{c}\Gamma_{\text {tot }}=9 A_{g}+6 A_{u}+6 B_{1 g}+9 B_{1 u}+ \\
9 B_{2 g}+6 B_{2 u}+6 B_{3 g}+9 B_{3 u}\end{array}$ \\
\hline $\mathrm{Na}(4 \mathrm{c})$ & $\mathrm{C}_{\mathrm{s}}: 2 \mathrm{~A}_{\mathrm{g}}+\mathrm{A}_{\mathrm{u}}+\mathrm{B}_{1 \mathrm{~g}}+2 \mathrm{~B}_{1 \mathrm{u}}+2$ & ${ }_{u}+B_{3 g}+2 B_{3 u}$ \\
\hline B (4c) & $\mathrm{C}_{\mathrm{s}}: 2 \mathrm{~A}_{\mathrm{g}}+\mathrm{A}_{\mathrm{u}}+\mathrm{B}_{1 \mathrm{~g}}+2 \mathrm{~B}_{1 \mathrm{u}}+2$ & $+B_{3 g}+2 B_{3 u}$ \\
\hline $\mathrm{H}_{1}(4 \mathrm{c})$ & $\mathrm{C}_{\mathrm{s}}: 2 \mathrm{~A}_{\mathrm{g}}+\mathrm{A}_{\mathrm{u}}+\mathrm{B}_{1 \mathrm{~g}}+2 \mathrm{~B}_{1 \mathrm{u}}+2$ & $u+B_{3 g}+2 B_{3 u}$ \\
\hline $\mathrm{H}_{2}(8 \mathrm{~d})$ & $\mathrm{C}_{1}: 3 \mathrm{~A}_{\mathrm{g}}+3 \mathrm{~A}_{\mathrm{u}}+3 \mathrm{~B}_{1 \mathrm{~g}}+3 \mathrm{~B}_{1 \mathrm{u}}$ & $3 B_{2 u}+3 B_{3 g}+3 B_{3 u}$ \\
\hline \multicolumn{2}{|c|}{ Monoclinic $\left(14 ; \mathrm{P} 2{ }_{1} / \mathrm{c} ; \mathrm{C}_{2 \mathrm{~h}}{ }^{5}\right)$} & $\Gamma_{\text {tot }}=3 A_{g}+9 A_{u}+3 B_{g}+9 B_{u}$ \\
\hline $\mathrm{Na}(2 \mathrm{a})$ & $\mathrm{C}_{\mathrm{i}}: 3 \mathrm{~A}_{\mathrm{u}}+3 \mathrm{~B}_{\mathrm{u}}$ & \\
\hline B (2b) & $\mathrm{C}_{\mathrm{i}}: 3 \mathrm{~A}_{\mathrm{u}}+3 \mathrm{~B}_{\mathrm{u}}$ & \\
\hline $\mathrm{H} \quad(4 \mathrm{e})$ & $\mathrm{C}_{1}: 3 \mathrm{~A}_{\mathrm{g}}+3 \mathrm{~A}_{\mathrm{u}}+3 \mathrm{~B}_{\mathrm{g}}+3 \mathrm{~B}_{\mathrm{u}}$ & \\
\hline
\end{tabular}




\section{CHAPTER 8. STRUCTURAL INVESTIGATION OF NaAIH 4 AND THE INFLUENCE OF BALL MILLING ON COMPRESSION PROPERTIES}

\subsection{Introduction}

Sodium alanate $\left(\mathrm{NaAlH}_{4}\right)$ has been proposed as a viable hydrogen storage medium capable of supplying hydrogen at moderate temperatures and at rates required for fuel cell applications. These favorable characteristics, particularly the reversibility of $\mathrm{NaAlH}_{4}$ as a hydrogen storage media, were reported first by Bogdanovic et al. [28]. An extensive study of related complex metal hydrides including the reversibility and enhanced desorption kinetics for $\mathrm{NaAlH}_{4}$ when doped with titanium and other transition metals followed [180-183]. Phase transitions and crystal structure modifications have been studied with the thermal decomposition of $\mathrm{NaAlH}_{4}[184,185]$. Enhanced desorption kinetics were clearly demonstrated for $\mathrm{NaAlH}_{4}$ doped with Ti and/or Zr catalyst [186, 187]. Ab initio calculations by projected augmented plane wave method indicate that $\mathrm{NaAlH}_{4}$ undergoes a phase transformation from tetragonal- P41/a to an orthorhombic$C m c 2_{1}$ structure at $6.43 \mathrm{GPa}$ with a $45 \%$ volume contraction [74]. It was also suggested that the orthorhombic phase of $\mathrm{NaAlH}_{4}$ stores more hydrogen volumetrically than the tetragonal phase and would, if stabilized at ambient conditions, be an interesting candidate for further studies with regard to hydrogen absorption/desorption efficiency. Talyzin et al. [188], performed high pressure Raman measurements of $\mathrm{NaAlH}_{4}$ up to 17 GPa. They reported two possible phase transformations: one around $3 \mathrm{GPa}$ which was less obvious as a structural transformation, and a second at $12 \mathrm{GPa}$ which was to an orthorhombic structure. Experimental study of Kumar et al. [73], reports a tetragonal to 
monoclinic phase transition above $14 \mathrm{GPa}$. Upon release of pressure, the tetragonal structure was found to reappear at $\sim 4 \mathrm{GPa}$. In this study, XRD measurements of unmilled or as received $\mathrm{NaAlH}_{4}$ showed the phase transformation to an orthorhombic structure which is in agreement with the prediction of Vajeeston et al. [74], followed by an amorphization at $13.6 \mathrm{GPa}$. A close analogue, $\mathrm{LiAlH}_{4}$, has a monoclinic structure at ambient conditions with space group $P 21 / c$ and undergoes a slow transition to a tetragonal phase between 2.2 and 3.5 GPa [69].

It has been reported that ball milling improves the hydrogen storage properties of hydrides including $\mathrm{NaAlH}_{4}[180,182,183,189-191]$. In general, the enhanced hydrogen sorption properties of hydrides after ball milling have been attributed to: the formation of large surface area, the reduction in grain size, and the introduction of lattice defects [192]. Milling essentially results in increasing the surface to bulk ratio, increasing grain and fault boundary densities that assist diffusion and the rate of $\mathrm{H}_{2}$ sorption. Therefore, it would be of great interest to study mechano-chemically milled $\mathrm{NaAlH}_{4}$ to understand the effect of milling on the structural behavior using high-pressure methods. To understand the possible structure relations with respect to processing conditions of $\mathrm{NaAlH}_{4}, \mathrm{X}$-ray diffraction and Raman spectroscopy measurements were performed on both unmilled and milled samples. In-situ measurements were carried out on unmilled $\mathrm{NaAlH}_{4}$ wet with mineral oil and found a structural transformation from a tetragonal to an orthorhombic phase above 8.6 GPa. Above $13 \mathrm{GPa}$, the sample shows an amorphous behavior. Ballmilled $\mathrm{NaAlH}_{4}$ wet with mineral oil was studied with micro X-ray diffraction using a synchrotron radiation source. Amorphization was started at $6.5 \mathrm{GPa}$ and completed at around $13.5 \mathrm{GPa}$ without any other phase transition. The intensity of the powder 
diffraction patterns decreased with increasing pressure. The structural transformation was found to be reversible when the pressure was released.

\subsection{Unmilled $\mathrm{NaAlH}_{4}$}

\subsubsection{Experimental details}

In-situ powder XRD and Raman spectroscopy measurements were carried out simultaneously on the $\mathrm{NaAlH}_{4}$ sample of $95 \%$ purity purchased from Sigma Aldrich. A sample wet with mineral oil was used for the study because of high reactivity of the $\mathrm{NaAlH}_{4}$ towards moisture. The sample was loaded along with tiny ruby chips in a DAC of culet size $600 \mu \mathrm{m}$. Sample chamber was a steel gasket with a hole size of $300 \mu \mathrm{m}$ and thickness $100 \mu \mathrm{m}$. XRD was carried out using a diffractometer equipped with rotating anode generator (Bruker) and facilitating radiation of wavelength $0.71073 \AA$. The 2D diffraction data were collected using a CCD detector.

\subsubsection{Results and discussion}

\subsubsection{X-ray diffraction}

At ambient conditions, the $\mathrm{NaAlH}_{4}$ has a tetragonal structure with space group I41/a. The lattice parameters of the unmilled sample were found to be $a=5.02629(2) \AA$ and $c=11.3562(7) \AA$, which gives a unit cell volume of $286.898(4) \AA^{3}$ that is in good agreement with the previous report [193]. Figure 8.1 shows the angle dispersive X-ray diffraction pattern of $\mathrm{NaAlH}_{4}$ obtained at various pressures upto $15 \mathrm{GPa}$. It can be noted from XRD results that the phase transformation begins at $8.6 \mathrm{GPa}$ and completes at 
around 12.4 GPa. The appearance of new peaks at high pressures between 8.6 and 9.5 $\mathrm{GPa}$ is a clear indication of the phase transition.

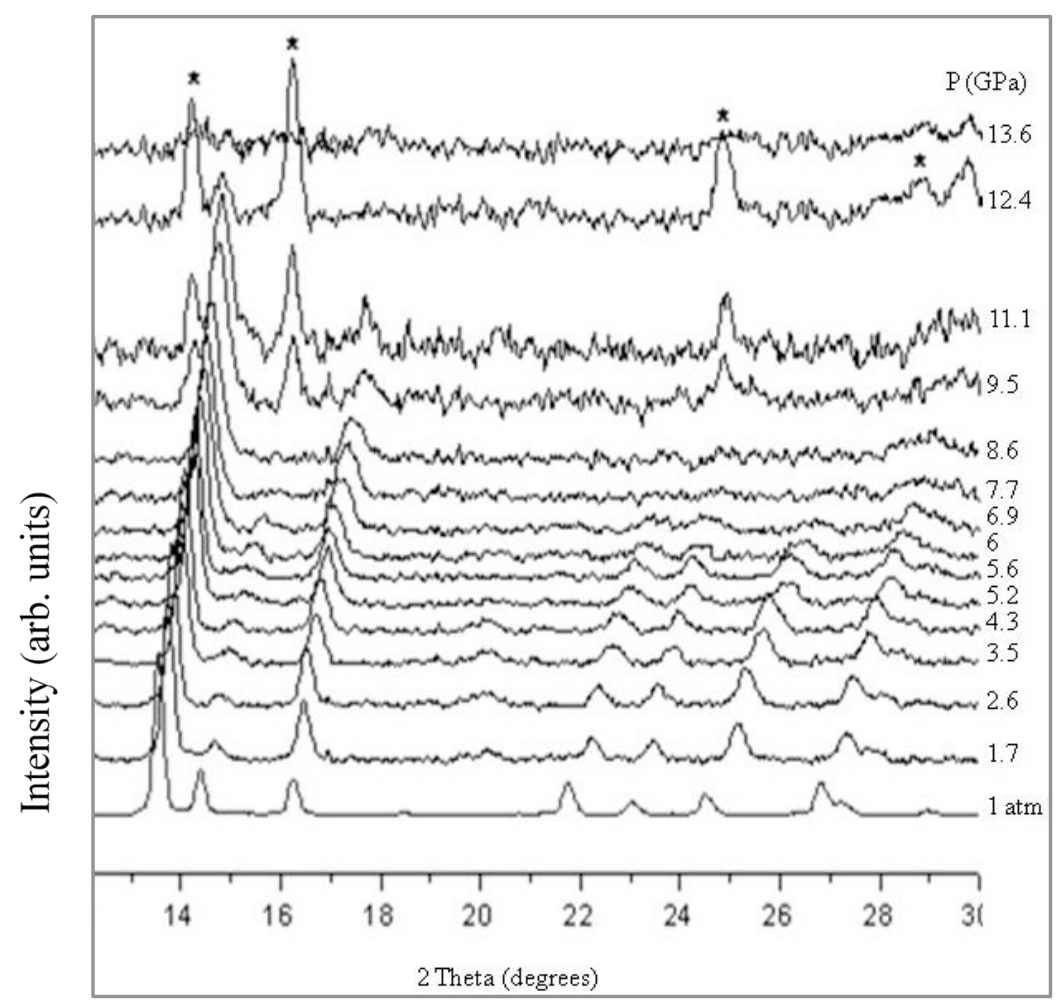

Figure 8. 1: The XRD patterns of unmilled $\mathrm{NaAlH}_{4}$ recorded at various pressures up to 13.6 GPa. Peaks due to orthorhombic phase are marked by asterisks at $12.4 \mathrm{GPa}$.

It was not possible to obtain the structural parameters above $6.5 \mathrm{GPa}$ because of poor quality of diffraction patterns. The signals became very weak at higher pressures and only two diffraction lines, which can be assigned to $\left(\begin{array}{lll}1 & 0 & 3\end{array}\right)$ and $\left(\begin{array}{lll}2 & 0 & 0\end{array}\right)$ atomic planes were observed indicating partial amorphization of the sample [194]. A material can go to an amorphous phase instead of a high pressure phase due to the strong stress effect [195]. Variation of lattice parameters with pressure was determined by structure refinement 
using Le Bail method. Figure 8.2 shows the obtained P-V plot of unmilled $\mathrm{NaAlH}_{4}$ fitted with third order Birch- Murnaghan EoS.

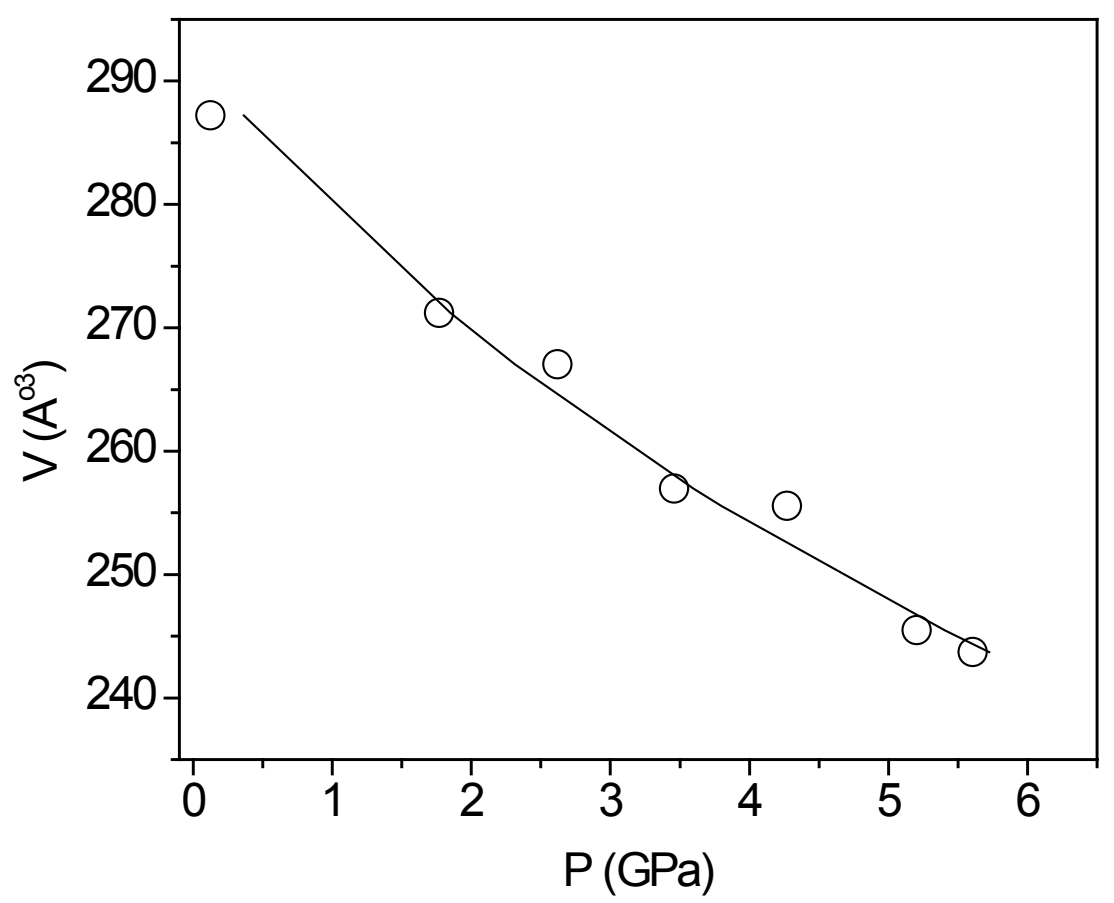

Figure 8. 2: The P-V plot of unmilled $\mathrm{NaAlH}_{4}$ (circles) fitted with Birch- Murnaghan equation of state (line).

The compression along c- axis was found to be three times that along a- axis of the unit cell of $\mathrm{NaAlH}_{4}$. The experimental data were fitted with third order BirchMurnaghan equation of state. Bulk modulus was found to be $25.65 \mathrm{GPa}$ with $\mathrm{K}_{0}{ }^{\prime}$ constrained to 4 and $\mathrm{V}_{0}=288.3 \AA^{3}$. Transition to an orthorhombic phase was observed just before amorphization. The transition pressure observed in this study was $2 \mathrm{GPa}$ above the computational result of Vajeeston et al. [74]. Amorphization of unmilled $\mathrm{NaAlH}_{4}$ loaded with mineral oil was observed between 12.4 and $13.6 \mathrm{GPa}$. The structural parameters were not refined for the orthorhombic- $\mathrm{NaAlH}_{4}$, but the peak positions shows 
orthorhombic structure with space group $\mathrm{Cmc} 2{ }_{1}$ similar to that predicted by Vajeeston et al. [74].

\subsubsection{Raman spectroscopy}

Raman spectrum of $\mathrm{NaAlH}_{4}$ obtained at ambient conditions with mode assignment was reported by Ross et al. [196]. Peaks observed in the range of 1600 and $1900 \mathrm{~cm}^{-1}$ are of the Al-H stretching modes, and that between 700 and $950 \mathrm{~cm}^{-1}$ is due to Al-H bending modes, whereas those vibrations lower than $600 \mathrm{~cm}^{-1}$ show the librational and torsional modes which are in good agreement with the literature [74, 188, 196]. Figure 8.3 shows Raman spectra collected at various pressures up to $15 \mathrm{GPa}$ during compression of unmilled $\mathrm{NaAlH}_{4}$.

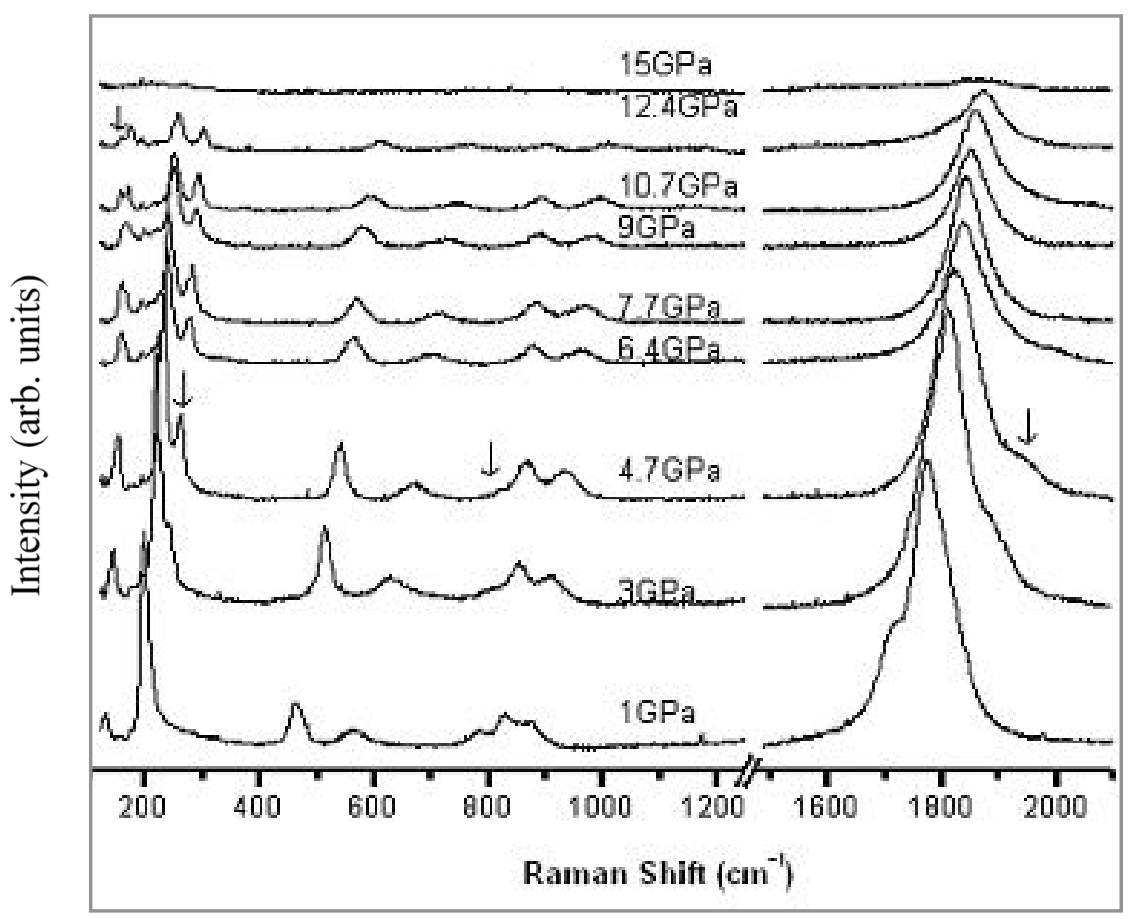

Figure 8. 3: Raman spectra of unmilled $\mathrm{NaAlH}_{4}$ at various pressures up to $15 \mathrm{GPa}$. The change in Raman modes under pressure are marked with arrow. 
From the Raman spectroscopy data, it is evident that the sample becomes amorphous between 12.4 and $15 \mathrm{GPa}$. A change in the peaks at liberational and stretching mode regions was observed around $3 \mathrm{GPa}$ which are indicated by arrows in Figure 8.3. Raman spectra show changes in the collective vibration modes such as the splitting of the peak at $130 \mathrm{~cm}^{-1}$, and separation of the shoulder at $250 \mathrm{~cm}^{-1}$ and appearance of a peak at $1685 \mathrm{~cm}^{-1}$. Above $3 \mathrm{GPa}$, the splitting of peak at $210 \mathrm{~cm}^{-1}$ is very prominent and which separates out completely at $\sim 12.4 \mathrm{GPa}$. Also, splitting of a peak in the region of collective vibration is observed between 9 and $12.4 \mathrm{GPa}$. The $\mathrm{Al}-\mathrm{H}$ stretching mode disappears at $1700 \mathrm{~cm}^{-1}$ and reappears at $1800 \mathrm{~cm}^{-1}$. The changes in the vibrational modes of unmilled $\mathrm{NaAlH}_{4}$ with pressure are evident from the Raman spectrum which confirms the phase transformation. The anharmonicities observed by Raman spectroscopy below 3 GPa may be attributed to an inhomogeneous pressure distribution within the sample or the local bonding structure change due to larger compression along c-axis as compared to a- axis. In comparison to the X-ray diffraction data, it is evident that the changes in the Raman spectrum around $3 \mathrm{GPa}$ corresponds to strong changes in the vibrational frequencies caused by distortion of the tetragonal structure and not a phase transition. The experiments were repeated three times with fresh loading of the sample and confirmed the occurrence of the orthorhombic phase around $9 \mathrm{GPa}$.

\subsection{Mechano-chemically Milled $\mathrm{NaAlH}_{4}$}

\subsubsection{Experimental details}

Powder sample of $\mathrm{NaAlH}_{4}$ having $99 \%$ purity was purchased from SigmaAldrich, and was ball-milled for 60 min in a Spex 8000 ball mill machine. A vial with 
stainless steel balls of diameters 6.35 and $12.7 \mathrm{~mm}$ are used to mill the sample at room temperature under a dry argon atmosphere. Milling typically resulted in a reduction of particle size without decomposition. The crystal structure and purity of the milled alanate were checked by XRD. The high-pressure micro X-ray diffraction experiments were performed at 16-IDB at HPCAT of Argonne National Laboratory. The monochromatic beam of wavelength $0.45657 \AA$ was focused to approximately $100 \mu \mathrm{m}^{2}$ and the data was recorded by a Mar345 image plate.

The sample was loaded with mineral oil in a Mao-Bell type DAC. A stainless steel gasket with a hole of size $200 \mu \mathrm{m}$ and thickness $50 \mu \mathrm{m}$, was used as the sample chamber. Grains of platinum metal were loaded with the sample as a pressure marker. Analysis of the XRD patterns of the ball-milled sample shows single phase $\mathrm{NaAlH}_{4}$ having tetragonal structure with space group $I 41 / a$ identical to the unmilled sample. In order to determine particle size, $\mathrm{LaB}_{6}$ crystals of particle size $2 \mu \mathrm{m}$ were used as the standard. XRD patterns of $\mathrm{NaAlH}_{4}$ (both as-received and ball-milled) and $\mathrm{LaB}_{6}$ were recorded at the same conditions. Lattice parameters were determined from Rietveld structure refinement using GSAS. Refined value of Lorentzian-Scherrer broadening term in the GSAS profile function of $\mathrm{LaB}_{6}$ was used as an instrumental broadening parameter. This value was subtracted from the corresponding broadening term of the $\mathrm{NaAlH}_{4}$ samples to determine diffraction line broadening related to the particle size effect.

\subsubsection{Results and discussions}

Gomes et al. [189], reported decreasing of the unit cell volume and c/a ratio after ball milling which is in agreement with our results. These observations can be attributed to the increase of surface to volume ratio in the particles. Average particle size (X) was 
determined using the relation: $X=\frac{18000 K * \lambda}{\pi L_{x}}$; Where $K$ is the Scherrer constant (0.9), $\lambda$ is the wavelength of X-rays in $\AA$, and $L_{x}$ is the Lorentzian broadening term in GSAS profile function. From the above equation, particle size of as-received and ball-milled $\mathrm{NaAlH}_{4}$ samples were found to be $1.08 \mu \mathrm{m}$ and $715 \mathrm{~nm}$, respectively. It was found that ball milling of the $\mathrm{NaAlH}_{4}$ decreases the particle size by $\sim 30 \%$. Figure 8.4 shows the XRD patterns of the ball milled $\mathrm{NaAlH}_{4}$ collected at various pressures up to $13.5 \mathrm{GPa}$.

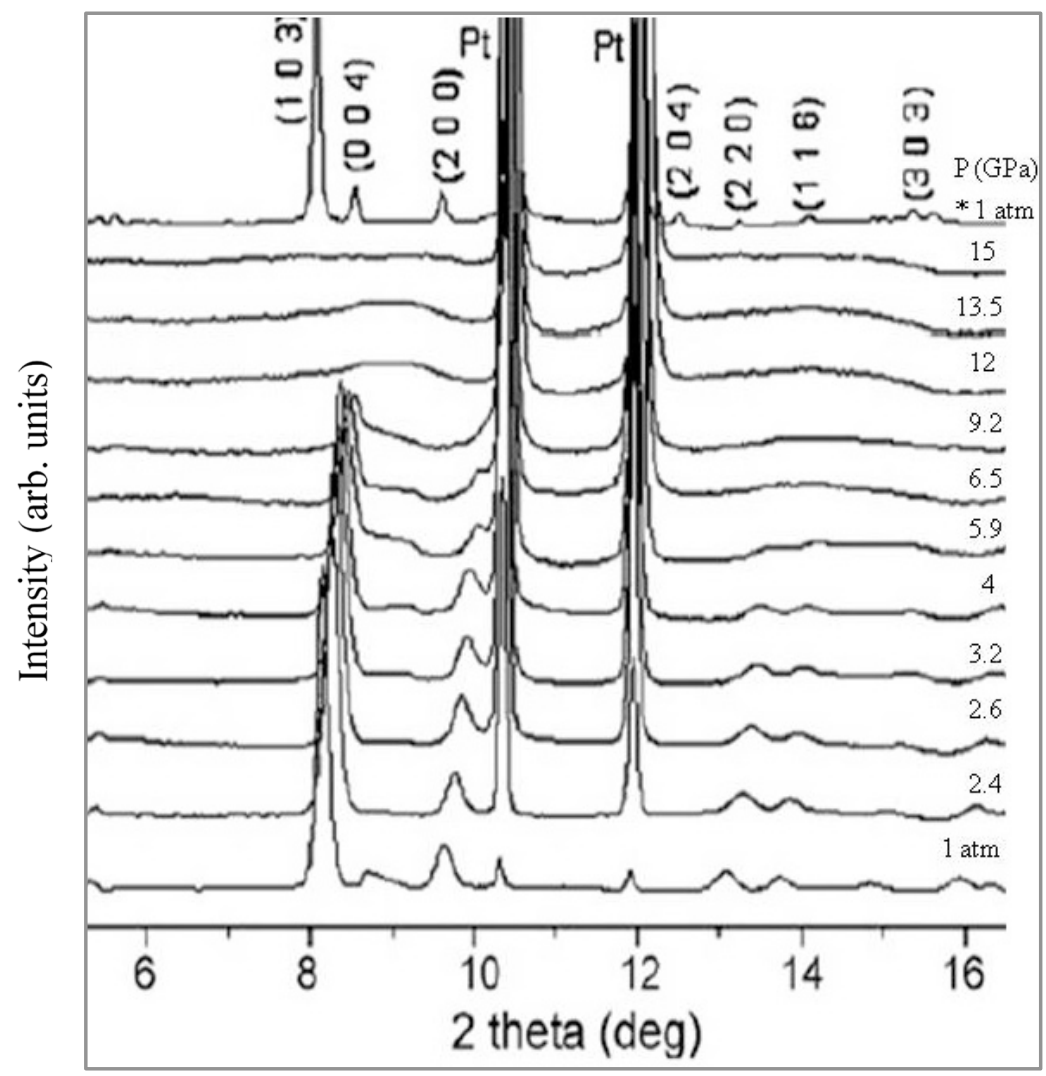

Figure 8. 4: The XRD patterns of the ball-milled $\mathrm{NaAlH}_{4}$ collected at various pressures. Pattern marked * is from decompressed sample, and Pt metal was used as a pressure marker. 
Lattice parameters of the ball milled $\mathrm{NaAlH}_{4}$ are determined by Rietveld refinement of the micro XRD data and found to be: $\mathrm{a}=4.800(1) \AA ; \mathrm{c}=10.56(1) \AA$ which gives a unit cell volume of $243.48(2) \AA^{3}$. From the analysis of the XRD data it is observed that the lattice parameters decrease with increasing pressure with a $c / a$ ratio of $\sim 2.2$ is maintained. The amorphization of the ball-milled sample was occurred at approximately $12 \mathrm{GPa}$, whereas for unmilled $\mathrm{NaAlH}_{4}$ it was at $13.6 \mathrm{GPa}$. Structural refinement was not possible at high pressures $>6.8 \mathrm{GPa}$, due to the peak broadening and disappearance of all Bragg reflections other than that of $\left(\begin{array}{lll}0 & 1 & 3\end{array}\right)$ and $\left(\begin{array}{lll}1 & 1 & 2\end{array}\right)$. This is an indication of the onset of amorphization of milled $\mathrm{NaAlH}_{4}$. The previous report of XRD study of $\mathrm{NaAlH}_{4}$ indicates a phase transformation to a monoclinic phase which corresponds to the amorphization pressure in our observation. This pressure-induced amorphization must have been influenced by the presence of mineral oil, which can be explained by the variation of the hydrostatic conditions [197]. High-pressure phases were absent in the ball-milled samples as observed from both the micro XRD and Raman spectroscopy experiments. The increase in the surface area (with strong stress effects) to bulk volume ratio, resulting from ball milling could have forced the system to an amorphous phase, as an easier path during the phase transformation [195].

The experimental P-V data were fitted with a third order Birch- Murnaghan equation of state using the Marquardt-Levenberg least square procedure with a sigma plot program. Figure 8.5 shows the behavior of unit cell volume with pressure and the EoS fit with $\mathrm{K}_{0}{ }^{\prime}$ constrained to 4 . The ball-milling of $\mathrm{NaAlH}_{4}$ caused an increase of $\mathrm{K}_{0}$ from 25.3(1) to 52.16(1) GPa, which is a surprisingly large positive effect. This can be attributed to the lattice defects induced during milling. The experiment was repeated two 
times to confirm the amorphization pressure and presence of the high-pressure phase. The difference in properties of ball milled sample from that of milled $\mathrm{NaAlH}_{4}$ may be caused by the size-induced pressure effect due to larger surface area in the material when compared to their bulk or due to the lattice defects generated during milling [198, 199]. To check the size effect with pressure, milled $\mathrm{NaAlH}_{4}$ was loaded with mineral oil and the XRD pattern was recorded up to $11.45 \mathrm{GPa}$. The pressure was then released to $2 \mathrm{GPa}$ and once again increased to $13.5 \mathrm{GPa}$ but did not observe any changes other than the amorphization. No high-pressure phase was observed prior to amorphization contrary to the unmilled $\mathrm{NaAlH}_{4}$. Hence, it can be concluded that pressurizing the sample to $11 \mathrm{GPa}$ did not have any effect on the particle size.

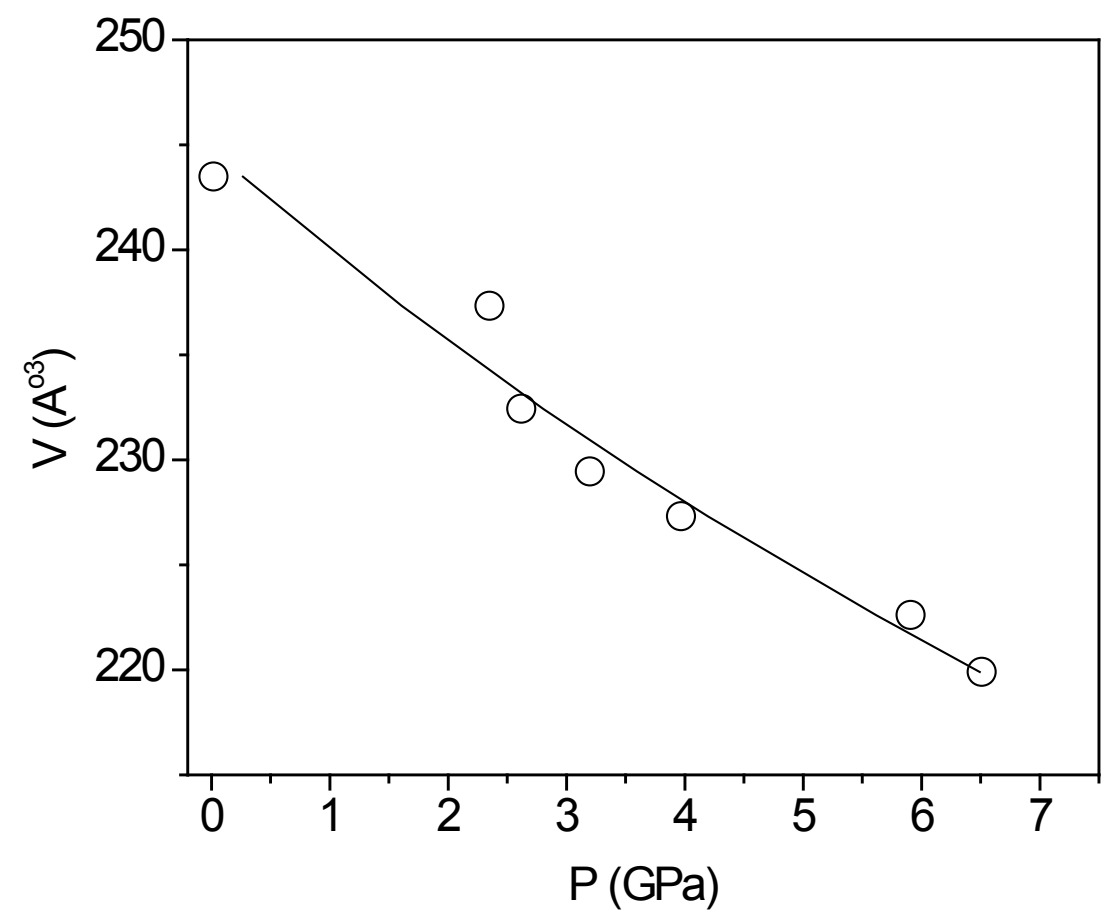

Figure 8. 5: Birch Murnaghan EoS fit (line) to the $P-V$ data (circles) of ball milled $\mathrm{NaAlH}_{4}$ 


\section{CHAPTER 9. HIGH PRESSURE INVESTIGATION OF STRUCTURAL STABILITY OF LiAIH}

\subsection{Introduction}

High-pressure modification of lithium alanate $\left(\mathrm{LiAlH}_{4}\right)$ reported to be a very interesting material for hydrogen storage applications, if it could be stabilized upon release of pressure [71]. $\mathrm{LiAlH}_{4}$ and $\mathrm{NaAlH}_{4}$ have received considerable attention due to their high theoretical hydrogen storage capacities and light weight [200]. Under ambient conditions $\mathrm{LiAlH}_{4}$ has a monoclinic structure with space group $P 21 / c$ and four formula units per unit cell [201]. The structure consists of $\mathrm{AlH}_{4}{ }^{-}$units separated by $\mathrm{Li}^{+}$ions, and the hydrogen atoms are arranged around the aluminum atoms in an almost regular tetrahedral configuration. According to the computation results of Vajeeston et al. [71], the monoclinic phases of $\mathrm{LiAlH}_{4}$ transforms above $2.6 \mathrm{GPa}$ into a tetragonal- I41/a structure which is isostructural to that of the ambient phase of $\mathrm{NaAlH}_{4}$ with a volume collapse of $17 \%$. Again, at $33.8 \mathrm{GPa}, \mathrm{LiAlH}_{4}$ undergoes a transition from tetragonal to an orthorhombic- Pnma structure [71].

Existing experimental data on high-pressure phase transformations of $\mathrm{LiAlH}_{4}$ are relatively old and were obtained using ex-situ methods [72, 202]. High- pressure Raman spectroscopy experiments of $\mathrm{LiAlH}_{4}$ by Talyzin and Sundqvist [70], and Chellappa et al. [69], confirmed a phase transition between 2 and 3.5 GPa. However, compression studies of $\mathrm{LiAlD}_{4}$ using neutron diffraction by Pitt et al. [203], showed no transitions up to 7.15 $\mathrm{GPa}$, and a two-phase mixture was observed on increasing temperature above this pressure. A reversible phase transition at ambient temperature was impossible to observe 
in such experiments, but the low pressure monoclinic phase of $\mathrm{LiAlH}_{4}$ was recovered after pressure treatment up to $9 \mathrm{GPa}$. The tetragonal and orthorhombic phases reported in these early studies were observed only when high pressure was combined with hightemperature treatment. High pressure phase transitions of pure $\mathrm{LiAlH}_{4}$ investigated using in situ Raman spectroscopy and computation which shows that $\sim 3 \mathrm{GPa}$ the ambient monoclinic- $P 21 / c$ phase of $\mathrm{LiAlH}_{4}$ transform to a high pressure phase with a distorted $\mathrm{AlH}_{4}{ }^{-}$tetrahedron $[69,70]$. The phase transition is reversible below $1.4 \mathrm{GPa}$ on decompression. In this study, in situ XRD and Raman spectroscopy measurements were carried out on the $\mathrm{LiAlH}_{4}$.

\subsection{Experimental Details}

The powder sample of $\mathrm{LiAlH}_{4}$ was obtained from Sigma Aldrich. The sample used for the study was loaded in DAC with mineral oil to avoid its reaction to moisture. The X-ray diffraction pattern were collected in steps of pressure using a rotating anode generator (Brucker) with facilitating radiation of wavelength $0.71073 \AA$. Raman spectrum were recorded is steps of pressure up to $50 \mathrm{GPa}$. Ruby fluorescence method was used for the pressure measurement.

\subsection{Results and Discussion}

\subsubsection{X-ray diffraction}

The analysis of powder X-ray diffraction pattern of $\mathrm{LiAlH}_{4}$ collected at ambient conditions suggest that it has a monoclinic- P21/c structure with lattice parameters; $\mathrm{a}=$ 4.858(3) $\AA, \mathrm{b}=7.832(6) \AA, \mathrm{c}=7.909(2) \AA$ and $\beta=112.53^{\circ}$. A high pressure phase 
transition at room temperature was observed above 2.16 GPa. Figure 9.1 shows the XRD patterns collected up to $23 \mathrm{GPa}$ at some selected pressures during compression.

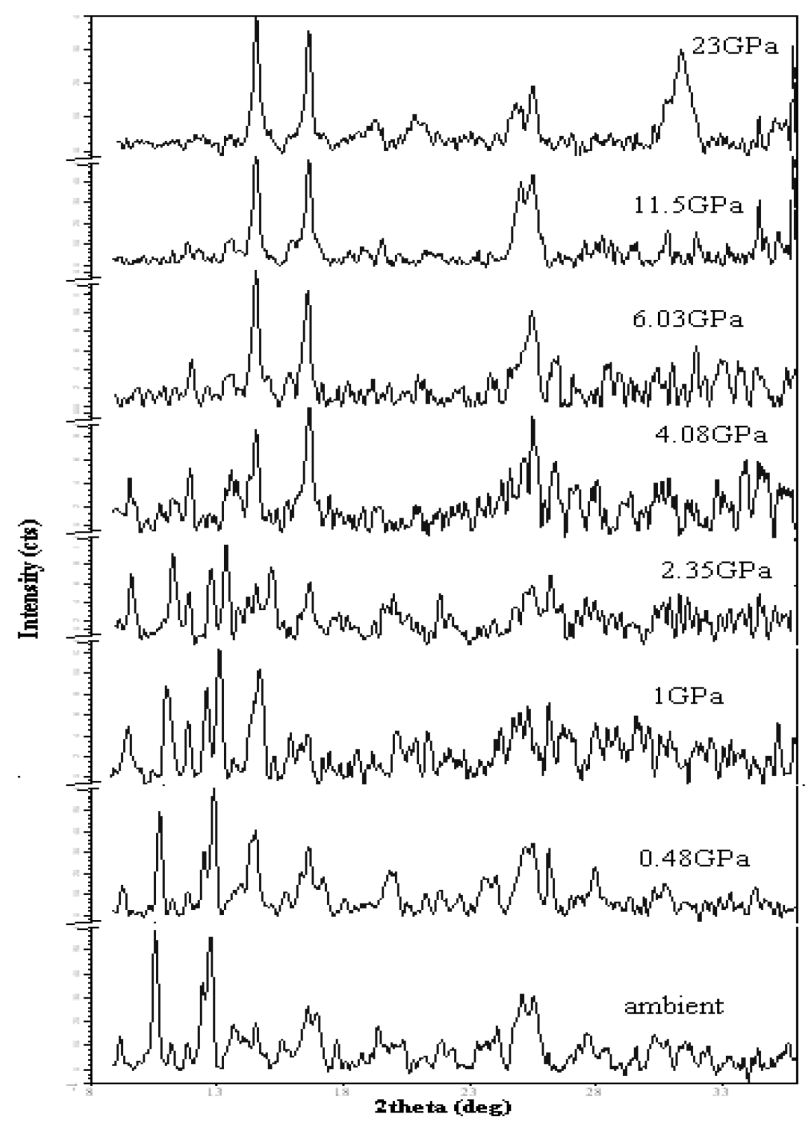

Figure 9. 1: Comparison of the high pressure XRD patterns of $\mathrm{LiAlH}_{4}$ collected at room temperature.

The change of unit cell volume with pressure has been determined from the analysis of XRD patterns. The P-V data thus obtained was fitted with third order BirchMurnaghan EoS (Figure 9.2). The bulk modulus of the ambient phase is found to be 13.87 GPa with a $\mathrm{K}_{0}^{\prime}=4$ and $\mathrm{V}_{0}=300.58 \AA^{3}$ which agree with the theoretical calculations [71]. 


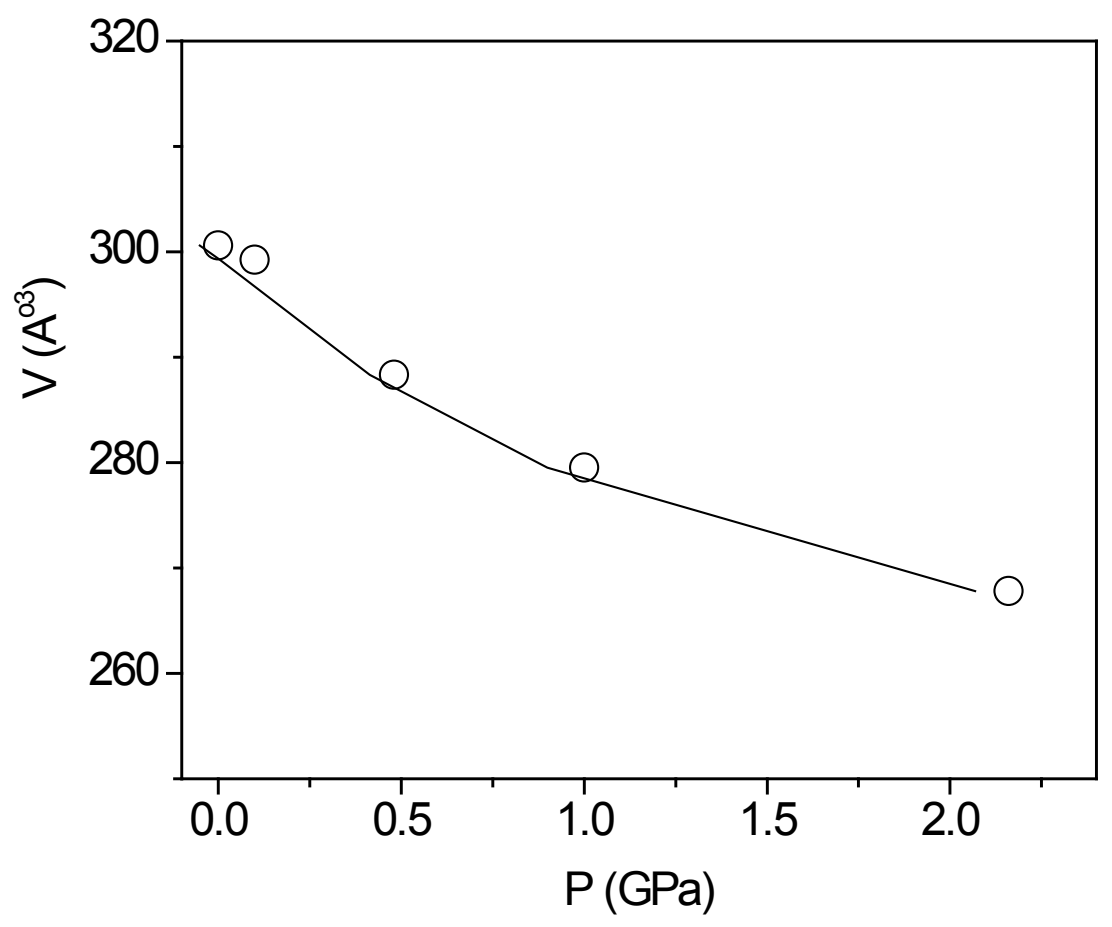

Figure 9. 2: The P-V data (circles) obtained for monoclinic phase of $\mathrm{LiAlH}_{4}$ fitted with third order Birch-Murnaghan EoS (line).

\subsubsection{Raman spectroscopy}

Figure 9.3 shows the Raman spectrum of monoclinic phase collected at ambient conditions. The strong characteristic peaks observed between 1600 and $1850 \mathrm{~cm}^{-}$ ${ }^{1}$ are due to $\mathrm{Al}-\mathrm{H}$ stretching modes. Al-H bending modes are found in the range 700-1500 $\mathrm{cm}^{-1}$. The liberational and translational lattice modes are very weak and observed between 270 and $550 \mathrm{~cm}^{-1}$. Raman spectra collected at high pressures show that a change in the vibrational modes occurs between 3.3 and $6.3 \mathrm{GPa}$. This can be attributed to a monoclinic to tetragonal structural phase transition. The phase transformation predicted by Vajeeston et al. [71], at $33 \mathrm{GPa}$ from computation was not observed in the Raman 
spectroscopic studies up to $50 \mathrm{GPa}$. Softening of Al-H stretching modes and corresponding shift to lower frequencies were observed beyond the transition pressure. The pressure quenched high pressure phase shows the Al-H stretching mode shift again to lower frequencies, suggesting a weakening of the Al-H bond. A weaker Al- $\mathrm{H}$ bond of the high pressure phase of $\mathrm{LiAlH}_{4}$ implies its structural instability. Therefore it is hypothesized that similar structural changes may be possible due to increased coordination number of $\mathrm{Al}^{3+}$ and the formation of $\mathrm{AlH}_{6}$ octahedra by ball milling of $\mathrm{LiAlH}_{4}$, which will result in enhanced dehydrogenation kinetics [69].

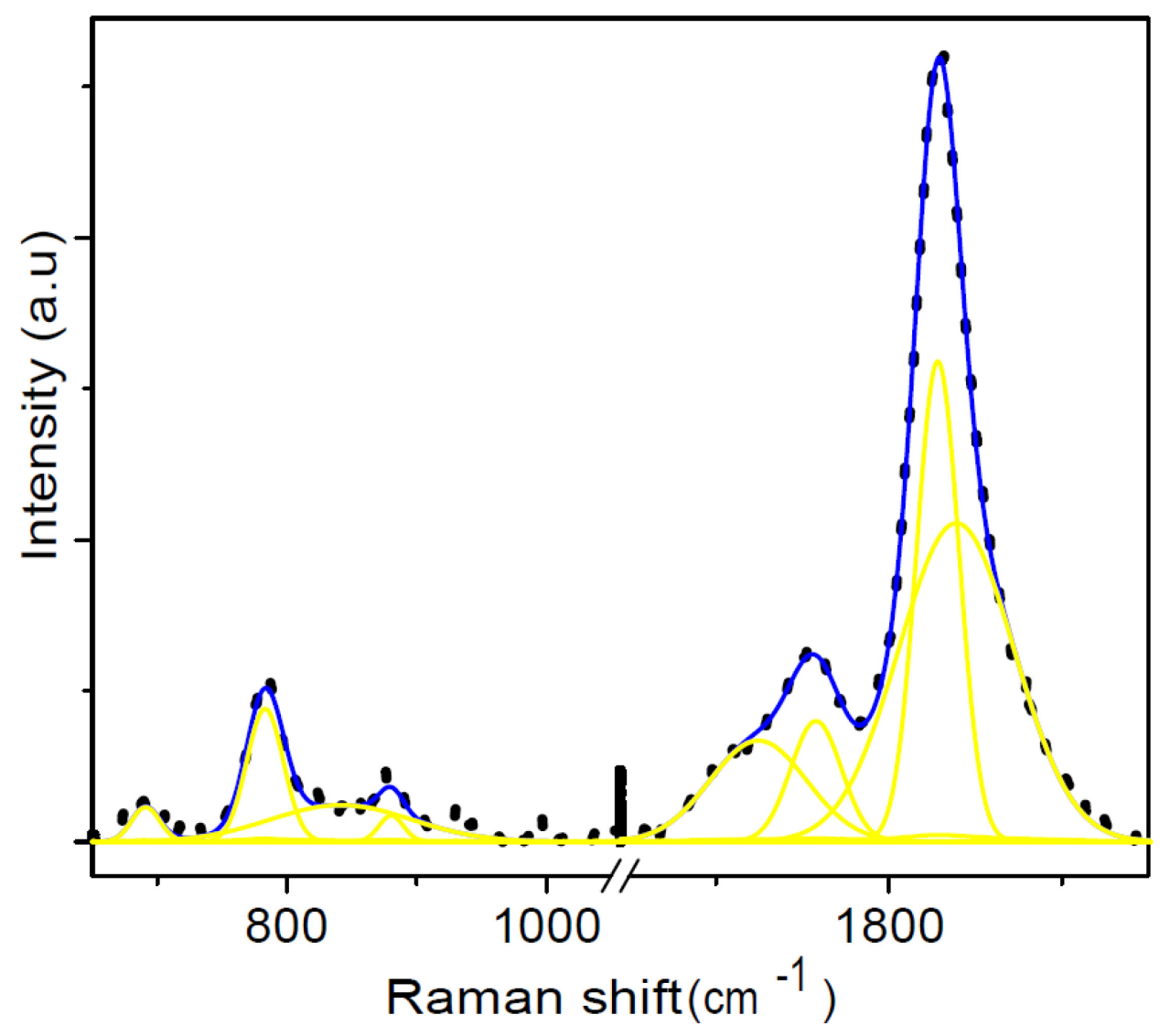

Figure 9. 3: Raman spectrum of $\mathrm{LiAlH}_{4}$ in at ambient conditions (dotted line) fitted with a Gaussian profile function. 
Figure 9.4 shows a comparison of the Raman spectra of $\mathrm{LiAlH}_{4}$ collected at various pressures up to $6.3 \mathrm{GPa}$. Between 3.3 to $6.3 \mathrm{GPa}$, the structural phase transition is evident from the appearance of a strong new peak at $1668 \mathrm{~cm}^{-1}$ and the disappearance of the Al-H stretching modes of monoclinic- $\mathrm{LiAlH}_{4}$.

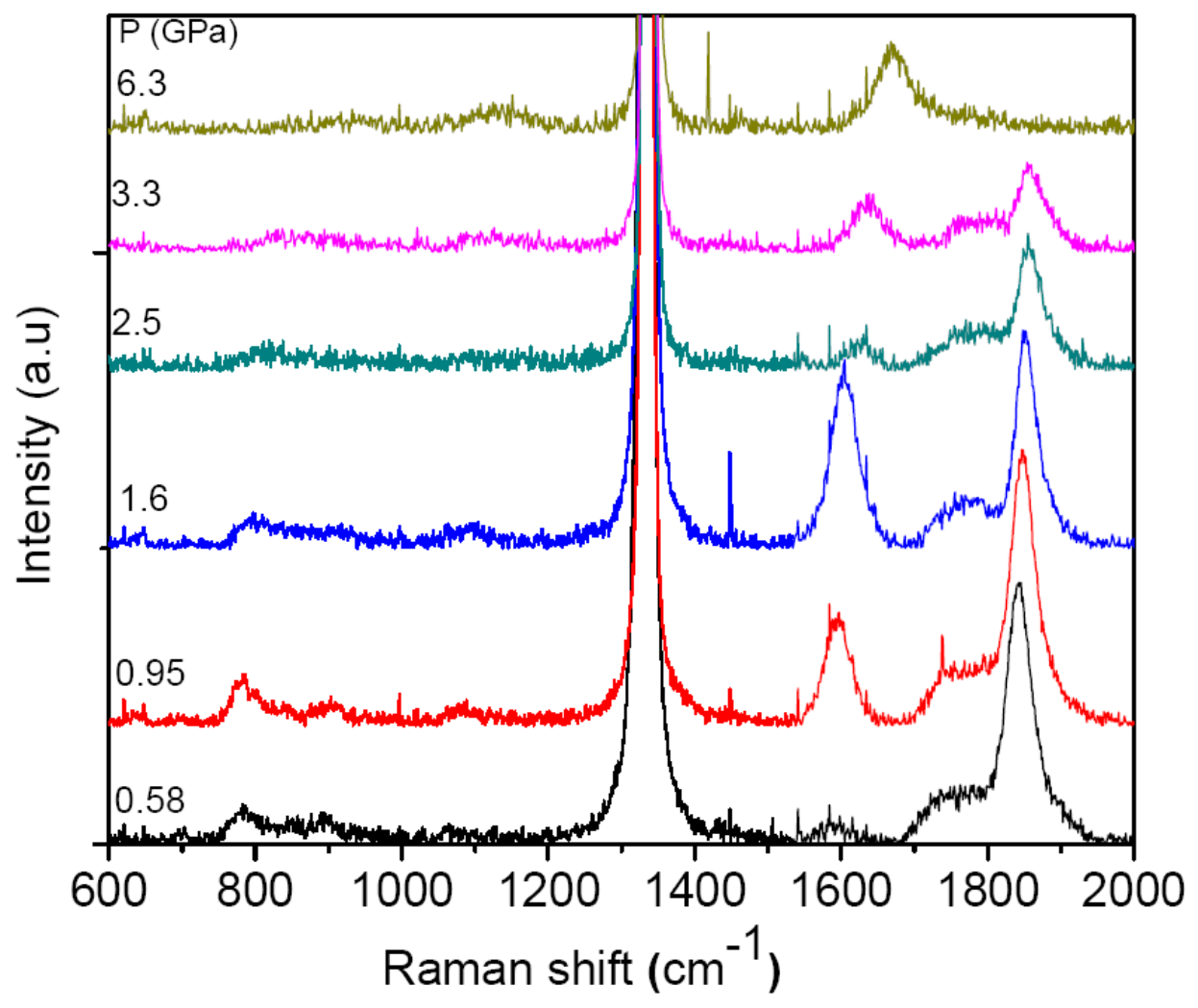

Figure 9. 4: Raman spectra of $\mathrm{LiAlH}_{4}$ collected at various pressures and at room temperature. 


\section{CHAPTER 10. PRINCIPAL COMPONENT ANALYSIS ON PROPERTIES OF BINARY AND TERNARY HYDRIDES}

\subsection{Introduction}

Material informatics is a developing area in material science in which a new knowledge system is built by collecting and classifying information with the help of calculations and databases. If experience and knowledge, captured and preserved in a database format, it helps to investigate new materials efficiently. The abundance of parameters causes difficulties in the interpretation of their relation. Many datum sets can be correlated to define a single material property with the help of a mathematical technique. The main challenge in this field is the difficulty in correlating many properties of different set of materials. Most of the classical analytical methods for finding characteristics of data set are very laborious and time consuming.

Lot of experimental and theoretical work has been done on hydrides in search of a suitable material. A detailed hydride research database is required for proper retrieval of information already available. Data were collected from available journal publications and constructed a property database. Data sets in the hydride database can be correlated to define a single material property using an available mathematical technique such as principal component analysis (PCA) which is a widely used tool for dimensionality reduction and for visualization of variable patterns. In this work, only binary (of the form $\mathrm{MH}_{\mathrm{x}}$, where $\mathrm{x}=1,2$ or 3 and $\mathrm{M}$ is a metal) and ternary (of the form $\mathrm{A}_{\mathrm{a}} \mathrm{B}_{\mathrm{b}} \mathrm{H}_{\mathrm{x}}$, where $\mathrm{a}, \mathrm{b}$ and $\mathrm{x}$ are integers and $\mathrm{A}, \mathrm{B}$ are metals) metal hydrides have been considered. Other 
hydrides were excluded from the study due to the presence of large missing data and associated error on analysis. This work investigates correlation among material properties using PCA method.

\subsection{Data}

The property data include but not limited to specific heat (Cp), entropy (S), molar volume (V) and hydrogen weight percentage ( $\mathrm{H}$ wt \%) of metal hydrides as well as the sum of elemental properties of the metals corresponds to each compound and constructed a database. Light metal hydrides of alkali and alkali-earth elements have good hydrogen storage capacity but operation temperature is very high while transition metal hydrides (eg. $\mathrm{LaNi}_{5} \mathrm{H}_{6}$ ) release $\mathrm{H}_{2}$ at room temperature but with less storage capacity. In order to understand property interrelationships of hydrides PCA have been carried out by considering the properties such as entropy of metal hydrides $\left(\mathrm{S}_{\mathrm{MH}}\right)$, molar volume of metal hydrides $\left(\mathrm{V}_{\mathrm{MH}}\right)$, sum of specific heat of metals $\left(\mathrm{Cp}_{\mathrm{M}}\right)$ in each hydride, specific heat of metal hydrides $\left(\mathrm{Cp}_{\mathrm{MH}}\right)$, sum of entropy of metals $\left(\mathrm{S}_{\mathrm{M}}\right)$ in each hydride, hydrogen weight per cent $\left(\mathrm{H} \mathrm{w}^{\circ}\right)$ of hydrides and sum of the molar volume of metals $\left(\mathrm{V}_{\mathrm{M}}\right)$ in each hydride. Only these properties have been included in the current study due to the unavailability of enough data on other properties.

\subsection{Method}

The property data of both binary and ternary hydrides are used for the study of their interrelationships. If there are large number of variables and some of which are correlated, it is required to consider only fewer variables that capture maximum variation 
in the original data set. For this purpose, a better approach is to consider all properties and carry out principal component analysis. PCA can reveal certain characteristics or combinations of the original variables that mostly determine the structure of the data distribution which may not be related to known influencing factors in the data set. PCA is one of the widely used techniques for dimensionality reduction with widespread applications to pattern recognition, data classification, exploratory data analysis, economics, life sciences, chemistry, geology and material science [204-208]. Due to fascinating interrelationships of material properties, use of PCA analysis has wide application in material science.

The data reduction in PCA is achieved by transforming variables to principal components, which are uncorrelated (orthogonal) and ordered so that the first few retain most of the variation in the total set of original variables with descending importance. If some of the variables in data set are correlated, only a minimum number of linear combinations are needed to describe most of the variability in the data set. Correspondingly, for less-correlated data sets, more combinations are necessary. Such linear combinations are called principal components (PC). As an example, if the first three components explain $\sim 90 \%$ of the variability among the original data set of 10 variables, the number of variables to be analyzed has been reduced from 10 to 3. In PCA the initial data matrix is represented as the inner product of row/ score matrix and column/ loading matrix. The row matrix has the dimensionality $\mathrm{r} \times \mathrm{n}$, where $\mathrm{r}$ is the number of observations (i.e., compounds) in the initial data set, and $\mathrm{n}$ is the number of principal components. The column matrix has the dimensionality $\mathrm{n} \mathrm{x} \mathrm{c}$, where $\mathrm{c}$ is the number of observable properties (variables) in the initial data set. In a plot of a score 
vector against another score vector, compounds with similar properties will cluster [209211].

PCA analyses have been carried out by forming a matrix with columns as hydride properties and rows corresponds to various hydrides. Based on the matrix diagonalization procedure in SPSS- 15 (Statistical Package for Social Sciences), principal components (eigenvectors) of the matrix were obtained. The eigenvectors are termed component loadings and they are used to calculate the component scores. PCA eigenvectors provide information about contribution of each variable to a component. This study helps to correlate variables having common behavior and hence to classify hydrides with respect to their chemical or physical nature.

The PCA option for factor analysis (FA) in SPSS is used in this study. FA is solely concerned with correlations among variables. The methodology of FA is very similar to PCA except that unique variability is excluded from the analysis. The output from the SPSS program is not identical to that of generally used PCA analysis. By default it will only extract factors whose eigenvalue is greater than one. By adjusting the number of factors equal to variables it is possible to overcome the difference between PCA and FA. Then the communalities (sum of the common components) are all 1.00 thus all the variability is assumed to be common. In SPSS, factor loadings (component matrix) are eigenvector normalized by the square root of its eigenvalue. The actual PCA can be found from the eigenvectors of the loadings by dividing each loading with the square root of the eigenvalue. The PCA derive a small number of linear combinations (PCs) of a set of variables that retain most of the information in the original variables. The projection of variables onto a PC is called scores. Plots of the scores for two or more PCs help us to 
find graphically the similarities and differences among variables. The distance between points in a score plot shows if they are similar or different [205]. Thus it may be able to find materials with similar properties (corresponds to closer points) which will help us to select new hydrides for particular applications.

Because of randomly distributed missing data in the dataset, pair wise deletion option is used in PCA to have a reasonable result. To achieve a clear pattern of coefficients, PC axes can be rotated in any direction without changing the relative locations of the points to one another with a change in actual coordinates of the points. The rotated solutions explain the same amount of variance as the original solution [212]. Varimax method of orthogonal rotation is an analytical way for rotation of PC solutions. The varimax rotation maximizes the sum of the variances of the squared coefficients within each eigenvector while the rotated axes remain orthogonal. The varimax normalised rotation has been carried out to maximize the values of the loading factors of each compound analyzed.

\subsection{Results and Discussion}

A data set of hydride properties with $\sim 200$ compounds is interpreted. PCA has been carried out on a set of data which consists of both binary and ternary hydrides as well as on sets of data which contain just binary or just ternary hydrides. Figure 10.1 shows eigenvalues and the variance explained by each principal components (PCs) obtained from the analysis of a set of data with both binary and ternary metal hydrides. Number of factors to be considered for extracting proper information is determined from the screeplot (plot of eigenvalue versus component number) and the percent variance. 
From the sudden fall of eigenvalues or the loadings of PCs (Figure 10.1) it is obvious that the first two PCs (which explains $62.89 \%$ and $30.15 \%$ variance, respectively) reduced the seven dimensional data set to a two dimensional data set, with an average $6.96 \%$ loss of detail.

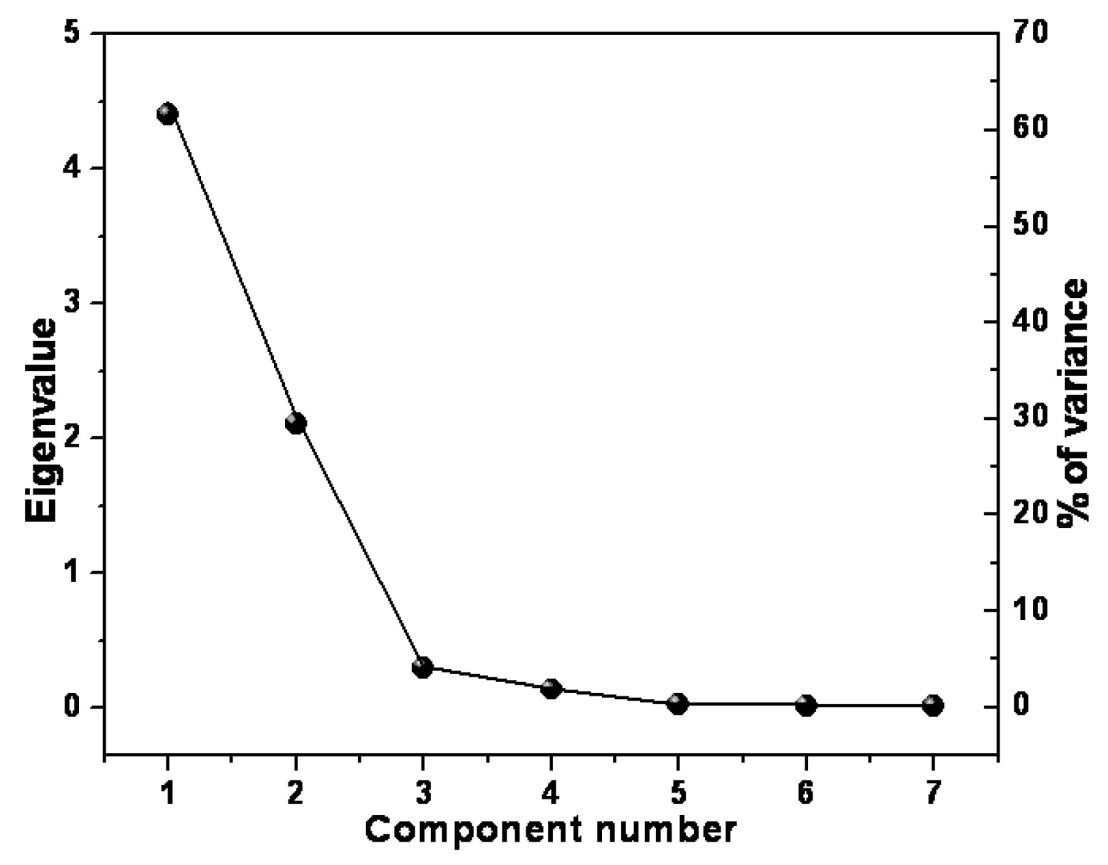

Figure 10. 1: Eigen value and total variance explained by each component obtained from PCA of a set of data with both binary and ternary metal hydrides.

The component matrix obtained after applying varimax rotation from PCA of a set of data which contain both binary and ternary hydrides is shown in Table 10.1. First column represent the variable and next seven columns represent eigenvectors correspond to the seven components. Contribution of first five variables to component one and last two variables to component two are considerable defining an arbitrary limit of 0.75 . The correlation matrix for this analysis is given in Table 10.2. In order to evaluate the 
correlation between variables, it is important to know the magnitude or strength of the correlation coefficient (Table 10.2) as well as the significance of the correlation. The results show correlations among $\mathrm{Cp}_{\mathrm{M}}, \mathrm{Cp}_{\mathrm{MH}}, \mathrm{S}_{\mathrm{MH}}$, and $\mathrm{V}_{\mathrm{MH}}$.

Table 10. 1: The component matrix obtained from PCA of a set of data with both binary and ternary hydrides. Subscript $\mathrm{M}$ denotes metal and $\mathrm{MH}$ abbreviates metal hydride.

\begin{tabular}{llllllll}
\hline Property & \multicolumn{7}{c}{ Components } \\
\cline { 3 - 7 } & 1 & 2 & 3 & 4 & 5 & 6 & 7 \\
$\mathrm{~S}_{\mathrm{MH}}$ & 0.984 & 0.085 & -0.086 & 0.004 & -0.120 & -0.024 & -0.033 \\
$\mathrm{~V}_{\mathrm{MH}}$ & 0.968 & -0.019 & 0.036 & -0.233 & 0.068 & 0.014 & -0.039 \\
$\mathrm{Cp}_{\mathrm{M}}$ & 0.935 & 0.216 & -0.185 & 0.192 & 0.074 & -0.051 & 0.002 \\
$\mathrm{Cp}_{\mathrm{MH}}$ & 0.872 & 0.471 & -0.013 & -0.110 & -0.019 & 0.013 & 0.070 \\
$\mathrm{~S}_{\mathrm{M}}$ & 0.759 & -0.628 & -0.022 & 0.143 & -0.001 & 0.087 & 0.003 \\
$\mathrm{Hwt} \%$ & 0.208 & 0.901 & 0.360 & 0.116 & 0.003 & 0.024 & -0.018 \\
$\mathrm{~V}_{\mathrm{M}}$ & 0.491 & -0.792 & 0.360 & 0.011 & -0.002 & -0.051 & 0.017 \\
\hline & & & & & & &
\end{tabular}

Table 10. 2: Correlation matrix obtained from PCA of a set of data with both binary and ternary hydrides. $\mathrm{M}$ and $\mathrm{MH}$ abbreviates metal and metal hydride, respectively.

\begin{tabular}{|c|c|c|c|c|c|c|c|}
\hline & $\mathrm{V}_{\mathrm{M}}$ & $\overline{S_{M}}$ & $\mathrm{Cp}_{\mathrm{M}}$ & $\overline{\mathrm{Cp}} \mathrm{p}_{\mathrm{MH}} \mathrm{V}$ & $\overline{\mathrm{AH}}$ & $\mathrm{H} \mathrm{wt} \%$ & $\mathrm{~S}_{\mathrm{MH}}$ \\
\hline$\overline{V_{M}}$ & 1.000 & 0.859 & 0.226 & 0.049 & 0.499 & -0.482 & 0.386 \\
\hline $\mathrm{S}_{\mathrm{M}}$ & 0.859 & 1.000 & 0.602 & 0.352 & 0.714 & -0.398 & 0.694 \\
\hline$C \mathrm{p}_{\mathrm{M}}$ & 0.226 & 0.602 & 1.000 & 0.897 & 0.855 & 0.343 & 0.948 \\
\hline$C \mathrm{p}_{\mathrm{MH}}$ & 0.049 & 0.352 & 0.897 & 1.000 & 0.857 & 0.587 & 0.899 \\
\hline $\mathrm{V}_{\mathrm{MH}}$ & 0.499 & 0.714 & 0.855 & 0.857 & 1.000 & 0.171 & 0.940 \\
\hline Hwt \% & -0.482 & -0.398 & 0.343 & 0.587 & 0.171 & 1.000 & 0.251 \\
\hline $\mathrm{S}_{\mathrm{MH}}$ & 0.386 & 0.694 & 0.948 & 0.899 & 0.940 & 0.251 & 1.000 \\
\hline
\end{tabular}


The plot of first two PCs obtained from PCA of a set of data which contain both binary and ternary hydrides is shown in the Figure 10.2. The selected properties are found to be confined to PC1-PC2 plane of the component plot which suggests that the first two components explain most of the variability in the data set as predicted from the screeplot.

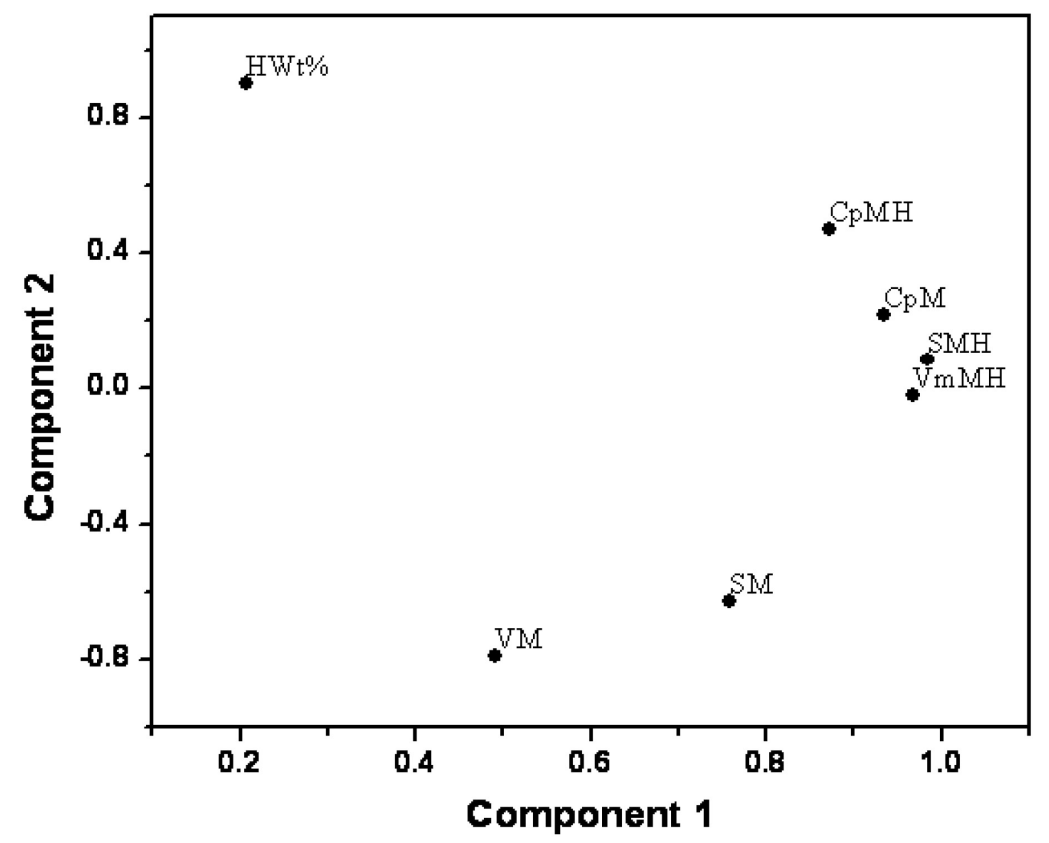

Figure 10. 2: Component plots for a set of data with both binary and ternary hydrides.

Subscript M denotes metal and MH denotes metal hydride. S- entropy, $\mathrm{Cp}$ - specific heat, $\mathrm{V}$ - molar volume and $\mathrm{H} \mathrm{wt} \%$ - hydrogen weight percent in each compound.

As two PCs cover $\sim 93 \%$ of the variance, plot of first two component scores is enough to visualize any possible patterns. From the score plot, shown in Figure 10.3, it can be observed that the ternary hydrides exhibits different phenomenon compared to binary hydrides. Therefore PCA of binary and ternary hydrides were carried out separately. 


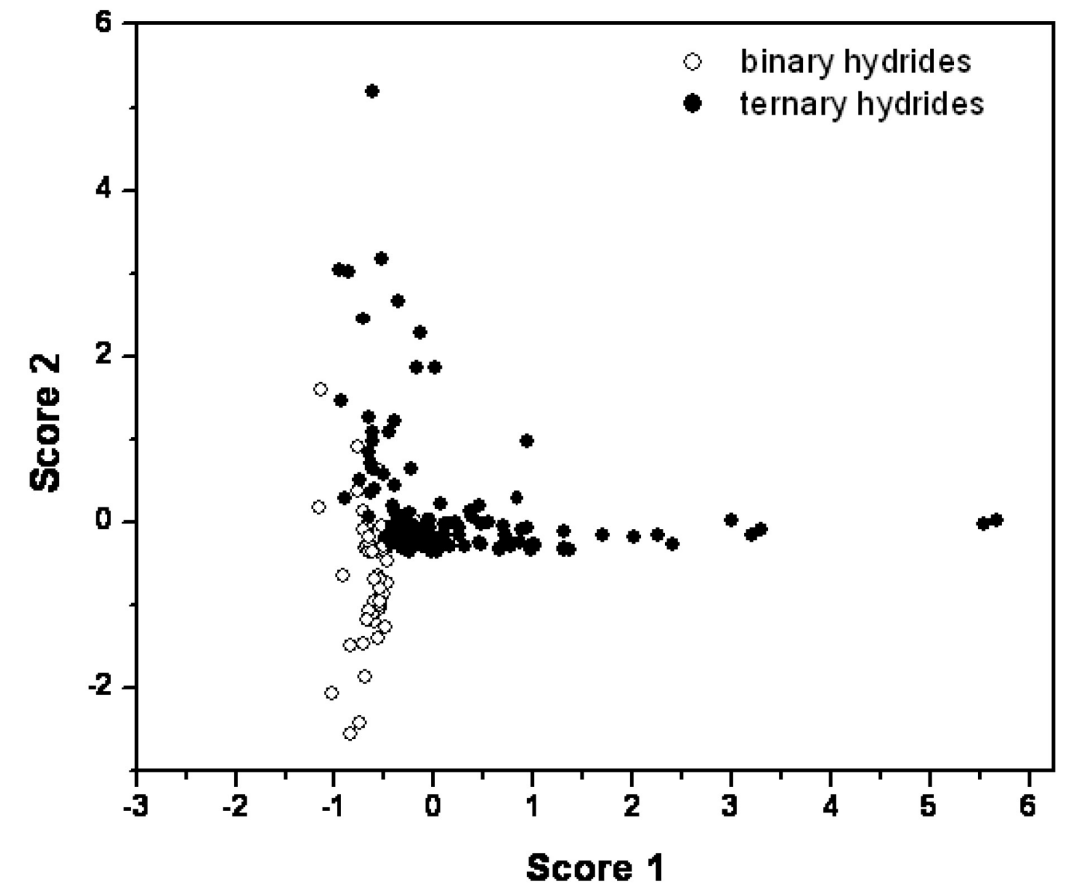

Figure 10. 3: Plot of score 2 versus score 1 of a set of data with both binary and ternary hydrides.

The score plots obtained are presented in Figures 10.4 and 10.5. The outliers found in these plots can be due to erroneous data for corresponding compounds. The score plots help us to select materials for specific applications from its position in the plot. The clustering of various hydrides in the score plots gives insight into similarity in their properties. If the PCA plots show interrelationship among many properties, it is not required to study all the properties of a hydride to determine its overall performance. 


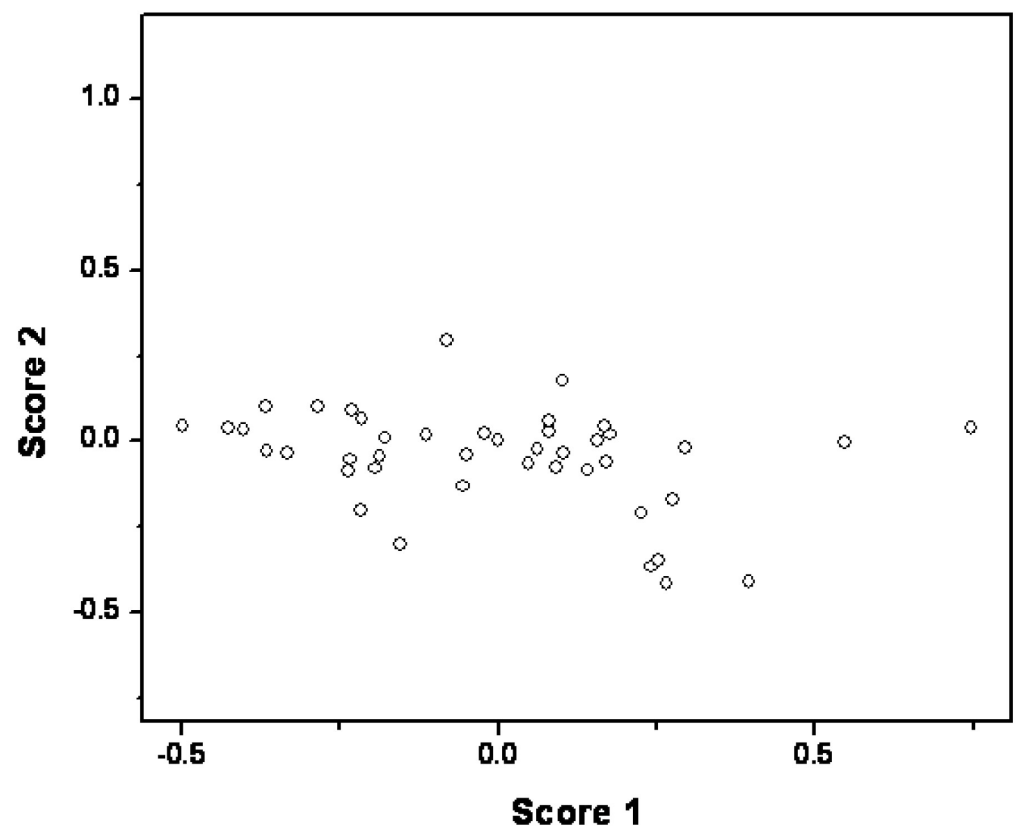

Figure 10. 4: Plot of scores of first two principal components for binary hydrides.

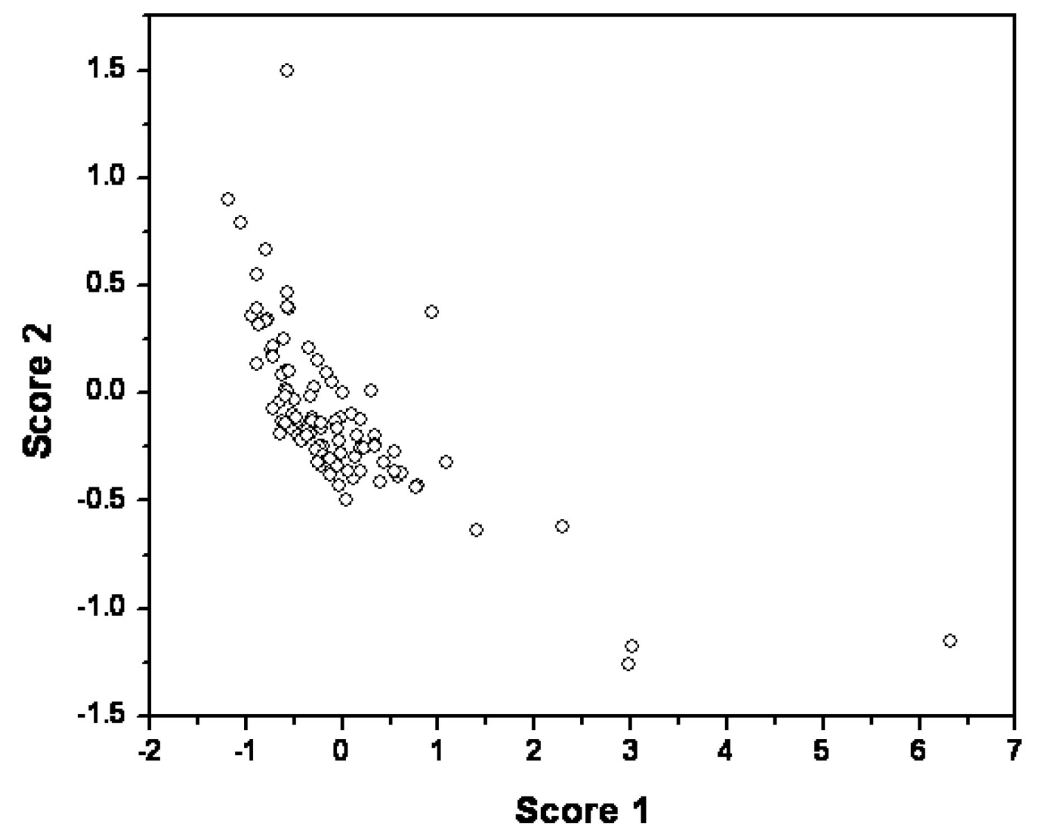

Figure 10. 5: Plot of scores of first two principal components for ternary hydrides. 


\section{CHPATER 11. INVESTIGATION OF MIXTURES OF HYDRIDES}

\subsection{High Pressure Raman Spectroscopic Investigation of $\mathrm{LiNH}_{2} \mathrm{BH}_{3}$}

\subsubsection{Introduction}

Ammonia borane, $\mathrm{NH}_{3} \mathrm{BH}_{3}$, is a promising candidate material for hydrogen storage with 19.6 wt $\%$ of $\mathrm{H}_{2}$. A thermal decomposition of pure ammonia borane proceeds in three steps and the release of all the hydrogen atoms occur only above 500 ${ }^{\circ} \mathrm{C}$. $\mathrm{NH}_{3} \mathrm{BH}_{3}$ releases hydrogen via catalyzed hydrolysis in an aqueous solution [213, 214], catalyzed dehydrocoupling under non-aqueous conditions $[215,216]$, or thermolysis at elevated temperatures [217, 218]. Catalyzed dehydrogenation, such as acid-catalyzed solvolysis significantly reduces gravimetric density of the material due to the presence of solvent. As a hydrogen storage material, $\mathrm{NH}_{3} \mathrm{BH}_{3}$ also face drawback of difficulty in its regeneration after dehydrogenation. Decomposition of pure $\mathrm{NH}_{3} \mathrm{BH}_{3}$ releases volatile byproducts like borazine that are unfavorable for fuel cell operation. The release of first equivalent of $\mathrm{H}_{2}$ from $\mathrm{NH}_{3} \mathrm{BH}_{3}$ typically occurs upon its melting at $110^{\circ} \mathrm{C}$. The subsequent release of the second equivalent of $\mathrm{H}_{2}$ occurs above $130{ }^{\circ} \mathrm{C}$, but is generally concurrent with the generation of borazine, $(\mathrm{BH})_{3}(\mathrm{NH})_{3}$, and diborane $\left(\mathrm{B}_{2} \mathrm{H}_{6}\right)$ gas impurities.

Xiangdong et al. [219, 220], reported that mechanically milling 1:1 mixture of ammonia borane and lithium hydride yields a destabilized hydrogen storage material, lithium amidotrihydroborate $\left(\mathrm{LiNH}_{2} \mathrm{BH}_{3}\right)$. It has a hydrogen storage capacity of around 10 wt $\%$. Advantage of $\mathrm{LiNH}_{2} \mathrm{BH}_{3}$ is that it can release over 7 wt $\%$ pure hydrogen at around $100{ }^{\circ} \mathrm{C}$, free of gas impurities. The decomposition temperature of the $\mathrm{NH}_{3} \mathrm{BH}_{3}$ 
sample destabilized by $\mathrm{LiH}$ was found to be lowered to $80{ }^{\circ} \mathrm{C}$. $\mathrm{LiNH}_{2} \mathrm{BH}_{3}$ has an orthorhombic- Pbca structure at ambient conditions [221]. The enthalpy of the reaction: $\mathrm{NH}_{3} \mathrm{BH}_{3}+\mathrm{LiH} \rightarrow \mathrm{LiNH}_{2} \mathrm{BH}_{3}+\mathrm{H}_{2} \uparrow$, is $-16 \mathrm{kcal} / \mathrm{mol}$ [222]. The decomposition reaction may be a two step process: $\mathrm{LiNH}_{2} \mathrm{BH}_{3} \rightarrow \mathrm{LiNHBH}_{2}+\mathrm{H}_{2} \rightarrow \mathrm{LiNBH}+\mathrm{H}_{2}$.

The lightest metal hydride, $\mathrm{LiH}$, has $12.5 \mathrm{wt} \%$ hydrogen content and $0.1 \mathrm{MPa}$ equilibrium hydrogen pressure at $910{ }^{\circ} \mathrm{C}$. The ambient phase has a cubic structure which is much compressible and densely packed [49]. Stephens and Lilley [51], investigated $\mathrm{LiH}$ with isotopic substitution at room temperature. High pressure experiments revealed that $\mathrm{LiH}$ is stable under compression up to $36 \mathrm{GPa}[52,223]$. The computationally predicted high pressure phase transition of $\mathrm{LiH}$ is at $0.66 \mathrm{TPa}$ [53]. Melting curve of $\mathrm{LiH}$ under compression was computed up to $200 \mathrm{GPa}$ and $2725^{\circ} \mathrm{C}$ by Ogitsu et al. [224], and observed a change in electronic structure of the compressed melt at high temperatures.

The $\mathrm{H}-\mathrm{H}$ interaction between the protonic $(\mathrm{N}-\mathrm{H})$ and hydridic $(\mathrm{B}-\mathrm{H})$ hydrogen atoms of adjacent molecules attributes to the high melting point of $\mathrm{NH}_{3} \mathrm{BH}_{3}$ as compared to similar compounds. Neutron diffraction studies at low-temperature show that in $\mathrm{NH}_{3} \mathrm{BH}_{3}$, the $\mathrm{N}-\mathrm{H} \ldots \mathrm{H}-\mathrm{B}$ interactions has the shortest $\mathrm{H} \ldots \mathrm{H}$ bond length of $2.02 \AA$. This is shorter than the the van der Waals distance $(2.4 \AA)$ between the $\mathrm{H}$ atoms. Under ambient conditions, $\mathrm{NH}_{3} \mathrm{BH}_{3}$ crystallizes in a tetragonal- $14 \mathrm{~mm}$ structure with a unit cell containing two molecules. At low temperature of about $-48{ }^{\circ} \mathrm{C}, \mathrm{NH}_{3} \mathrm{BH}_{3}$ exhibits a first order rotational order-disorder phase transition to an orthorhombic- $P m n 2_{1}$ structure [225]. Raman spectroscopy studies of $\mathrm{NH}_{3} \mathrm{BH}_{3}$ shows that the vibrational modes involving the $\mathrm{NH}_{3}$ group show negative pressure dependences whereas that of $\mathrm{BH}_{3}$ group have a positive shift, which support the existence of the dihydrogen bond [226]. 
Raman spectroscopy studies of $\mathrm{NH}_{3} \mathrm{BH}_{3}$ at high pressures by Lin et al. [227], observed N-H stretching modes in the range $3170-3320 \mathrm{~cm}^{-1}$. Changes in the N-H stretching region were occurred at 5,12 and 14.4 GPa during compression. The B-H stretching modes (2200 and $\left.2700 \mathrm{~cm}^{-1}\right)$ and B-N stretching modes $\left(700-1000 \mathrm{~cm}^{-1}\right)$ show transitions at 2 and $12 \mathrm{GPa}$ [227]. B-H bending vibrational modes was observed at 1171 and $1192 \mathrm{~cm}^{-1}$. The results suggest that $\mathrm{NH}_{3} \mathrm{BH}_{3}$ shows three phase transitions at approximately 2, 5, and $12 \mathrm{GPa}$ [227]. At $2 \mathrm{GPa}$, the asymmetric B-H stretching mode, the asymmetric B-H bending mode, as well as the low frequency rocking mode started to split into two modes. Around $5 \mathrm{GPa}$, coalescence of the asymmetric $\mathrm{N}-\mathrm{H}$ stretching mode, as well as the splitting of the combined $\mathrm{B}-\mathrm{H}$ and $\mathrm{N}-\mathrm{H}$ rocking modes occurred. It is proposed that $\mathrm{NH}_{3} \mathrm{BH}_{3}$ is dimerized at high pressure, forming diammonite of diborane, $\mathrm{H}_{2} \mathrm{~B}\left(\mathrm{NH}_{3}\right)_{2} \mathrm{BH}_{4}$. Ammonia borane shares the same empirical formula $\left(\mathrm{BNH}_{6}\right)$ with diamonite of borane and are products of symmetrical and unsymmetrical cleavages of borane by ammonia, respectively.

Chitra et al. [228], investigated pressure- induced structural transformations of diborane $\left(\mathrm{B}_{2} \mathrm{H}_{6}\right)$ using in situ Raman spectroscopy. Diborane is found to exist in the liquid phase up to pressures of less than $4 \mathrm{GPa}$. At $4 \mathrm{GPa}$, it undergoes a liquid- solid phase transformation to a new high- pressure phase with a possible structure similar to that of its low- temperature orthorhombic phase. Again, above $6 \mathrm{GPa}$ a new phase with a possible extended network structure of higher hydrides of borane has formed. At pressures above $14 \mathrm{GPa}$, diborane transforms to a third high-pressure phase. All of the observed pressure-induced structural transformations of $\mathrm{B}_{2} \mathrm{H}_{6}$ are completely reversible upon decompression. In the present study, $\mathrm{LiNH}_{2} \mathrm{BH}_{3}$ is investigated under pressure 
using in situ Raman spectroscopy experiments. Because of poor quality of Raman spectra collected in the bending mode region, only the B-H and N-H stretching modes are analyzed in detail.

\subsubsection{Results and Discussions}

Raman spectroscopy studies of $\mathrm{LiNH}_{2} \mathrm{BH}_{3}$ were conducted at ambient and high pressure conditions on both compression and decompression. Table 11.1 lists the vibrational modes observed for $\mathrm{LiNH}_{2} \mathrm{BH}_{3}$ at ambient conditions. Figures 11.1 and 11.2 shows the obtained Raman spectrum of $\mathrm{LiNH}_{2} \mathrm{BH}_{3}$ collected at ambient conditions from the sample loaded in a quartz capillary. In comparison to the computational results of the vibratonal modes by Lee et al. [229], it can be concluded that the Raman spectrum collected at ambient condition is that of pure $\mathrm{LiNH}_{3} \mathrm{BH}_{3}$. Because of low intensity of B-H and $\mathrm{N}-\mathrm{H}$ bending modes collected from DAC, only B-H and $\mathrm{N}-\mathrm{H}$ stretching regions are considered for further analysis. After loading the sample in a DAC, the Raman spectrum of the sample is different from the as synthesized phase which was confirmed in repeated experiments. Results show that the $\mathrm{LiNH}_{2} \mathrm{BH}_{3}$ sample just closed in DAC $(<0.1 \mathrm{GPa})$ undergoes partial transition/decomposition. Figure 11.3 shows images of the sample chamber at different pressures. Portion of the sample which is transparent (W) and opaque (D) to light are marked in the image at $4.2 \mathrm{GPa}$. 


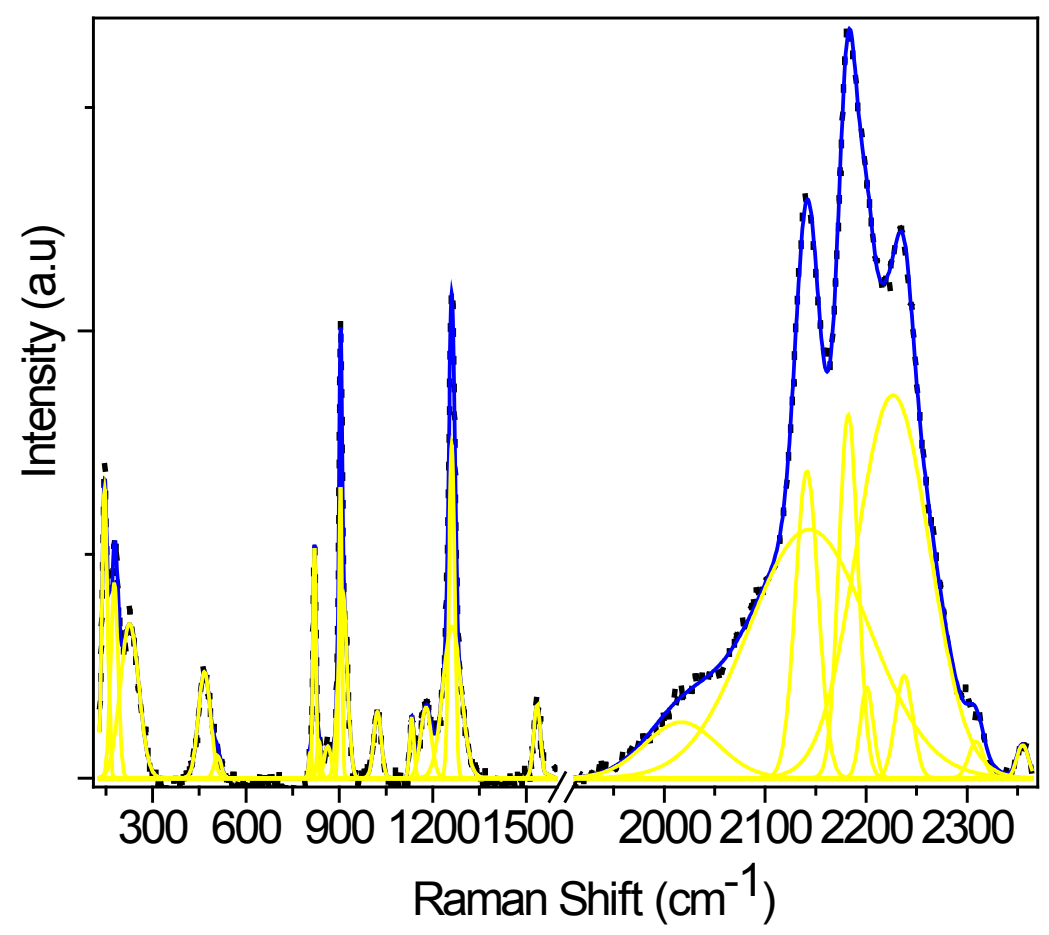

Figure 11. 1: Lattice, bending and B-H stretching modes in the Raman spectrum of $\mathrm{LiNH}_{2} \mathrm{BH}_{3}$, collected at ambient conditions fitted with a Gaussian profile function.

Figure 11.4 shows Raman spectra of the sample at different pressures collected from both $\mathrm{W}$ and $\mathrm{D}$ regions. The Raman shift frequencies of $\mathrm{B}-\mathrm{H}$ and $\mathrm{N}-\mathrm{H}$ stretching modes at $4.2 \mathrm{GPa}$ which corresponds to two different phase regions, D and $\mathrm{W}$, are listed in Table 11.2. The frequencies given in bold letters in the table can not be due to $\mathrm{LiNH}_{2} \mathrm{BH}_{3}$. The dark regions in the sample can be a two phase region which contains an unidentified phase along with $\mathrm{LiNH}_{2} \mathrm{BH}_{3}$. The high pressure phase transitions were reversible on decompression. The decompressed patterns show $\mathrm{LiNH}_{2} \mathrm{BH}_{3}$ in both $\mathrm{W}$ and D regions. 


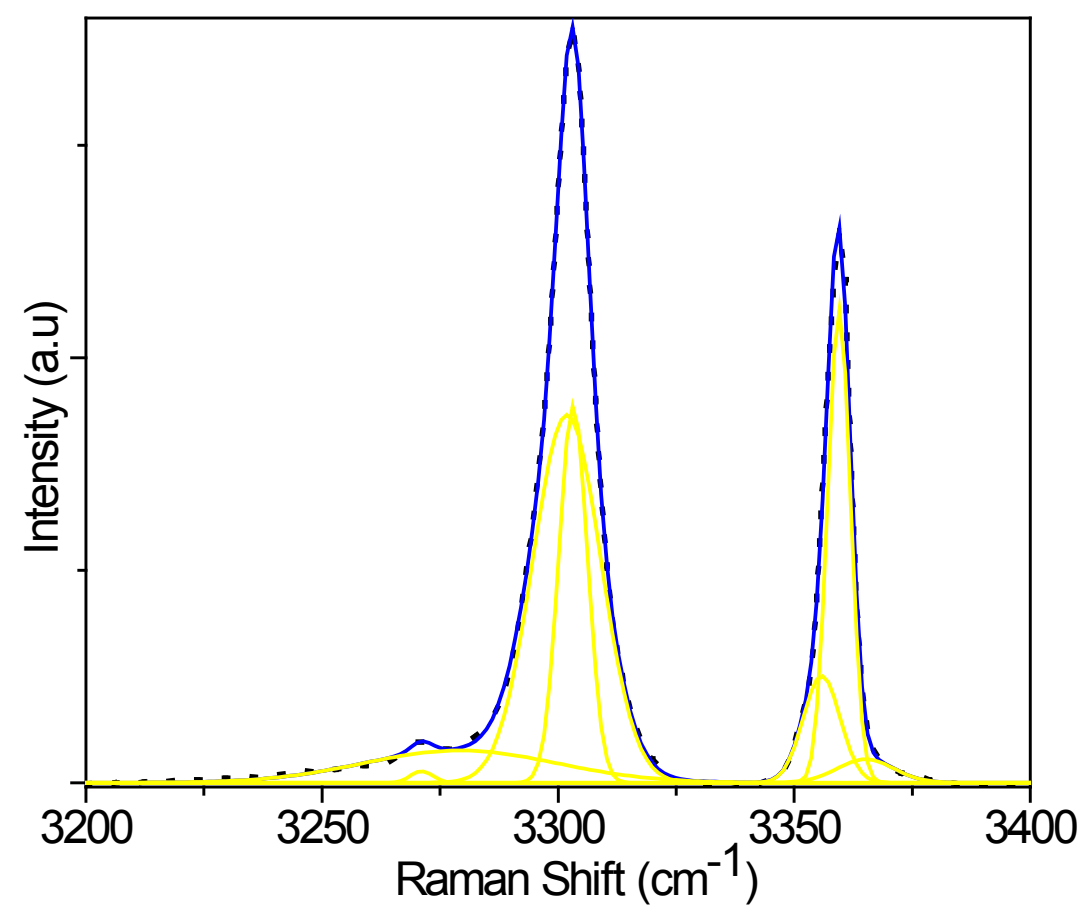

Figure 11. 2: N-H stretching modes in the Raman spectrum of $\mathrm{LiNH}_{2} \mathrm{BH}_{3}$, collected at ambient conditions fitted with a Gaussian profile function.

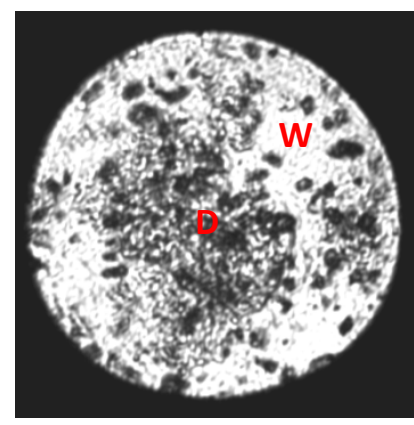

$4.2 \mathrm{GPa}$

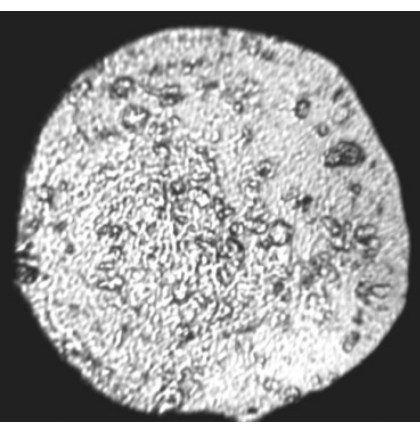

$17.2 \mathrm{GPa}$

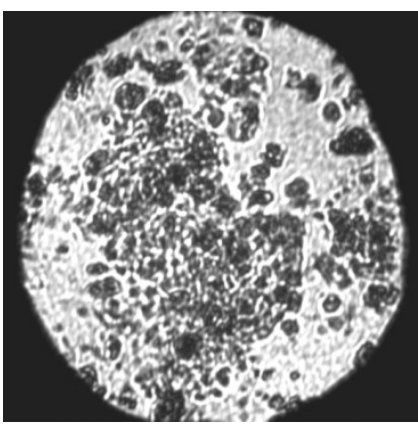

DP0

Figure 11. 3: The sample loaded in DAC with two phase regions marked W and D at different pressures. DP0 implies decompressed sample at ambient conditions. 

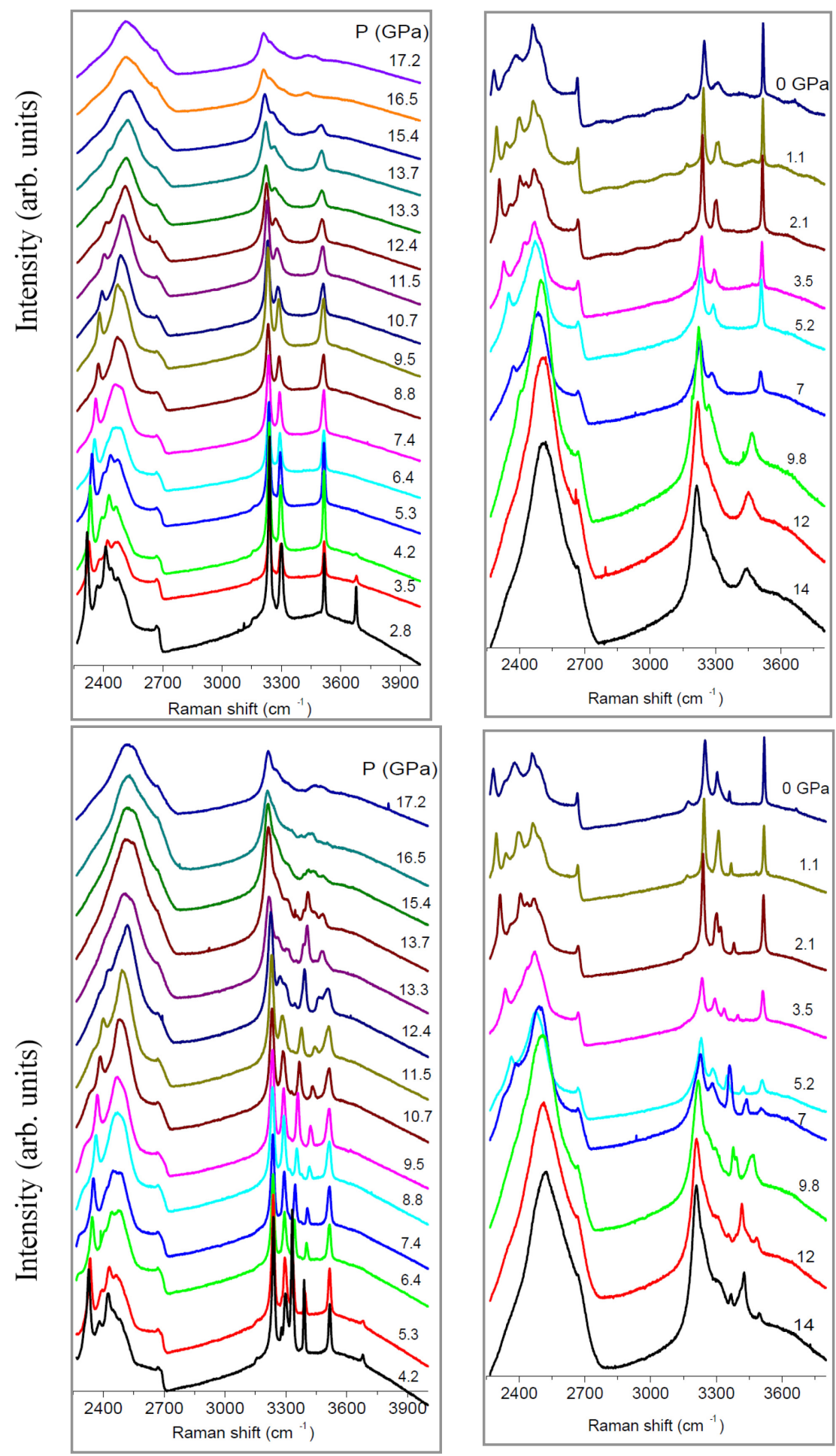

Figure 11. 4: Raman spectra of $\mathrm{LiNH}_{2} \mathrm{BH}_{3}$ during compression (right) and decompression (left) for the regions W (top) and D (bottom), respectively. 
Table 11. 1: Raman shift frequencies in $\mathrm{cm}^{-1}$ for $\mathrm{LiNH}_{2} \mathrm{BH}_{3}$ (LAB) in this study at ambient conditions in comparison with that of $\mathrm{NH}_{3} \mathrm{BH}_{3}(\mathrm{AB})$; str. Abbreviates stretching modes. The mode frequencies marked * are from FTIR study [229].

\begin{tabular}{|c|c|c|c|c|c|c|}
\hline $\mathbf{L A B}$ & $\begin{array}{c}\mathbf{A B} \\
{[230]}\end{array}$ & & $\mathbf{L A B}$ & $\begin{array}{l}\mathbf{A B} \\
{[230]}\end{array}$ & $\mathbf{L A B}$ & $\begin{array}{l}\mathbf{A B} \\
{[230]}\end{array}$ \\
\hline Bending/str. & & & B-H str. & B-H str. & N-H str. & N-H str. \\
\hline $\begin{array}{l}806.01 \\
800^{*}\end{array}$ & 727 & $\begin{array}{l}\text { N-B-H } \\
\text { rock }\end{array}$ & 2016.9 & $\mathrm{x}$ & $\begin{array}{l}3271.09 \\
3197 *\end{array}$ & $\begin{array}{l}3176, \\
3195^{*}\end{array}$ \\
\hline 819.968 & 784 & B-N str. & 2141.32, & $\mathrm{x}$ & $\begin{array}{l}3301.85 \\
3253 *\end{array}$ & $\begin{array}{l}3250 \\
3253^{*}\end{array}$ \\
\hline $\begin{array}{l}834.154, \\
842 *\end{array}$ & 800 & B-N str. & $\begin{array}{l}2143.72 \\
2148 *\end{array}$ & $\mathrm{x}$ & 3303.19, & $\begin{array}{l}3316, \\
3314^{*}\end{array}$ \\
\hline 862.465 & $\mathrm{x}$ & & $\begin{array}{l}2182.37 \\
2196^{*}\end{array}$ & $\mathrm{x}$ & $\begin{array}{l}3355.82 \\
3359 *\end{array}$ & \\
\hline $\begin{array}{l}903.248, \\
901 * \text { B-N str. }\end{array}$ & $\mathrm{x}$ & & 2200.69 & $\mathrm{x}$ & 3359.5 & \\
\hline 910.905 & $\mathrm{x}$ & & 2226.71 & $\mathrm{x}$ & $\begin{array}{l}3664.92 \\
3675^{*}\end{array}$ & \\
\hline $\begin{array}{l}1021.78, \\
1016^{*}, 1066^{*}\end{array}$ & 1065 & $\begin{array}{l}\text { N-B-H } \\
\text { rock }\end{array}$ & $\begin{array}{l}2237.66 \\
2287 *\end{array}$ & $\begin{array}{l}2279 \\
2287 *\end{array}$ & & \\
\hline 1132.58 & 1155 & $\mathrm{BH}_{3}$ & 2308.96 & 2328 & & \\
\hline $\begin{array}{l}\text { 1177.97, } \\
1177 * \text { B-H }\end{array}$ & 1189 & $\mathrm{BH}_{3}$ & $\begin{array}{l}2354.97 \\
2349 *\end{array}$ & $\begin{array}{l}2375 \\
2343 *\end{array}$ & & \\
\hline 1261.03 & 1357 & $\mathrm{NH}_{3}$ & & & & \\
\hline $\begin{array}{l}1261.31, \\
1261 *, \mathrm{~B}-\mathrm{H} \\
1381^{*}\end{array}$ & 1450 & ov & & & & \\
\hline $\begin{array}{l}1534.8, \\
1543^{*}, \mathrm{~N}-\mathrm{H} \\
1616^{*}\end{array}$ & 1600 & $\mathrm{NH}_{3}$ & & & & \\
\hline
\end{tabular}

The peak observed at $3664 \mathrm{~cm}^{-1}$ is very weak at ambient conditions, but is stronger in the Raman spectra collected from the $\mathrm{LiNH}_{2} \mathrm{BH}_{3}$ sample loaded in a DAC. Lee et al. [229], observed a peak at $3675 \mathrm{~cm}^{-1}$ in FTIR study which was unidentified. They proposed that it may be due to an impurity of $-\mathrm{OH}$ or $-\mathrm{NH}$ origin. It can not be due 
to a Li related mode because that is observed around $1380 \mathrm{~cm}^{-1}$. Other possibilities of origin of the peak at $3675 \mathrm{~cm}^{-1}$ was suggested to be due to disordered orientations of $\mathrm{NH}_{3}$ and $\mathrm{BH}_{3}$ groups in the sample or an effect of peak splitting due to crystal field. This peak disappears above $4.2 \mathrm{GPa}$ with the observed phase transition and hence it may conclude that the peak at $3664 \mathrm{~cm}^{-1}$ is of $\mathrm{LiNH}_{2} \mathrm{BH}_{3}$. The observed phase separation can not be due to inhomogeneus pressure distribution, as the opaque region is not only located at the center of the sample chamber but also at the corners of the gasket hole. The sharp ruby fluorescense spectra observed during compression also suggest somewhat hydrostatic conditions in the sample. High precausion was taken while loading the sample in the DAC in an argone atmosphere of the glove box. Therefore, reaction with moisture is least expected. Another possibility is the exposure to laser and the heating effect. This was sorted out after long time laser exposure to both transparent and opaque regions without any change. Therefore, it is expected that the phase separation is due to partial decomposition of $\mathrm{LiNH}_{2} \mathrm{BH}_{3}$. The Raman spectra at any pressure does not show any hydrogen peaks. Above $14 \mathrm{GPa}$, similar spectra was obtained from sample in both regions and the sample was found to be transparent to light above this pressure. Hence, it may be concluded that there may be a slow chemical reaction in the sample under pressure which completes around $14 \mathrm{GPa}$ and the sample become the unidentified new phase above this pressure. 
Table 11. 2: B-H and N-H stretching modes of the $\mathrm{LiNH}_{2} \mathrm{BH}_{3}$ in $\mathrm{cm}^{-1}$ at $4.2 \mathrm{GPa}$ as observed from Raman spectra of two different phase regions, D and W. The wave numbers given in bold are of an unidentified phase.

\begin{tabular}{llll}
\hline D & W & D & W \\
\hline 2309.61 & 2317.27 & 3157.22 & 3157.12 \\
2327.62 & 2332.94 & 3234.76 & 3234.13 \\
2329.96 & 2334.34 & 3239.16 & 3237.94 \\
$\mathbf{2 3 7 8 . 9 8}$ & $\mathbf{x}$ & $\mathbf{3 2 5 7 . 3 3}$ & $\mathbf{x}$ \\
2379.84 & 2392.01 & 3266.69 & 3270.21 \\
2421.87 & 2425.58 & 3296.16 & 3295.75 \\
2449.54 & 2466.52 & 3306.38 & 3309.17 \\
$\mathbf{2 4 8 6 . 4 8}$ & $\mathbf{x}$ & $\mathbf{3 3 3 1 . 1 5}$ & $\mathbf{x}$ \\
2511.57 & 2514.68 & $\mathbf{3 3 8 6 . 4 1}$ & $\mathbf{x}$ \\
& & $\mathbf{3 3 9 3 . 7 5}$ & $\mathbf{x}$ \\
& & 3513.64 & 3513.41 \\
& & 3516.23 & 3514.95 \\
& & 3679.03 & 3679 \\
\hline
\end{tabular}

Figure 11.5 shows the change of Raman shift frequency with pressure for both W and $\mathrm{D}$ regions. It may be concluded that the $\mathrm{W}$ portion is pure $\mathrm{LiNH}_{2} \mathrm{BH}_{3}$ up to $4.2 \mathrm{GPa}$ and above which it undergoes a phase transition. The $\mathrm{D}$ portion is a mixture of $\mathrm{LiNH}_{2} \mathrm{BH}_{3}$ and an unidentified phase. From the Raman shift versus pressure plots, the phase boundaries can be indentified at 4.2, 9 and $15 \mathrm{GPa}$ in $\mathrm{W}$ region. Whereas, in the D region, the phase boundaries can be marked between 11-12 and 14-15 GPa. In this experiment, Raman spectra were collected from the D region only above $4.2 \mathrm{GPa}$. A repeated experiment shows a phase transition of the D region below $5 \mathrm{GPa}$ as well. Therefore it may be summarized that $\mathrm{LiNH}_{2} \mathrm{BH}_{3}$ undergoe three phase transitions around 5, 10 and $14 \mathrm{GPa}$. 

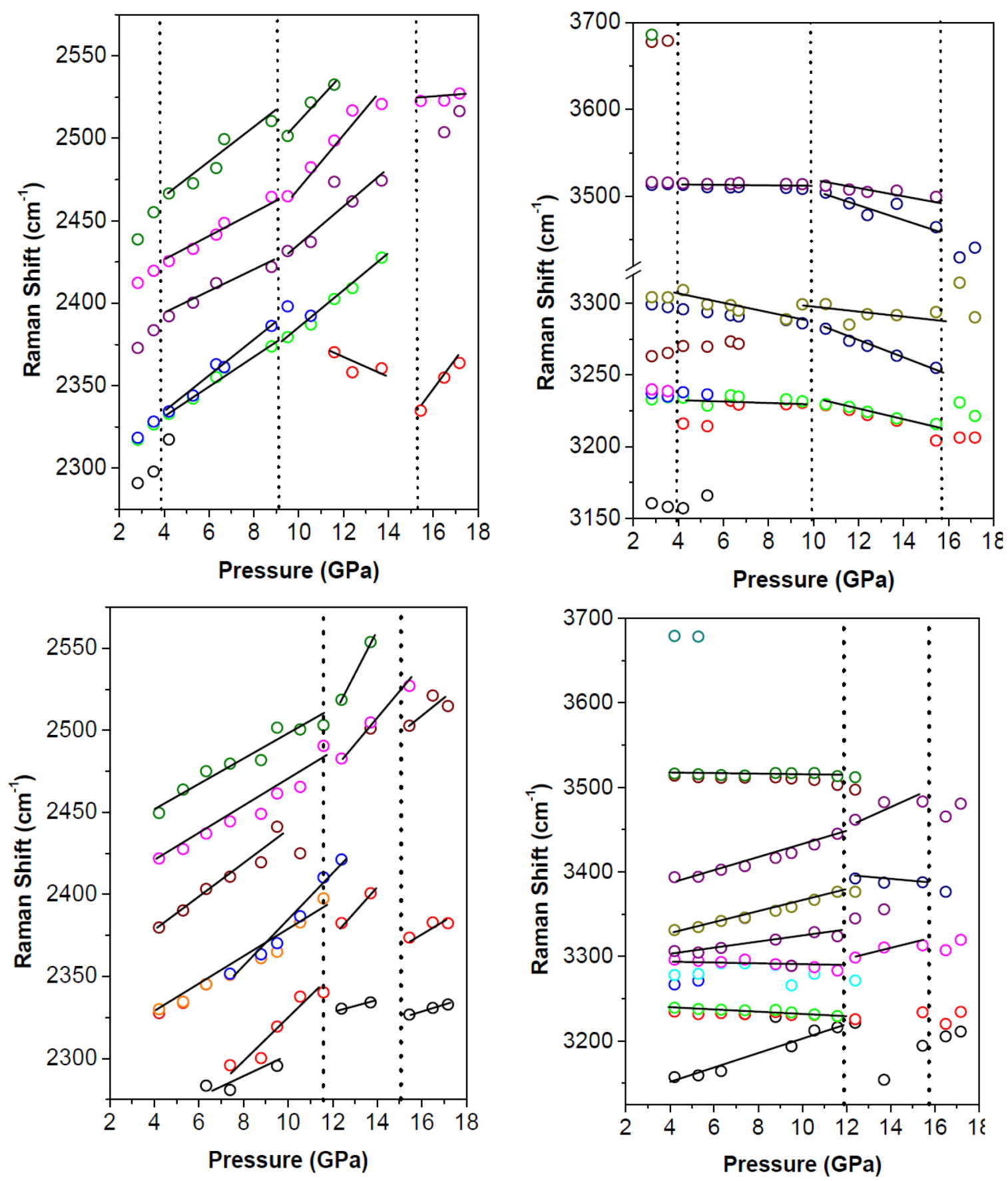

Figure 11. 5: Change of Raman shift with pressure for $\mathrm{LiNH}_{2} \mathrm{BH}_{3}$ during compression of B-H (left) and N-H (right) stretching modes for the regions W (top) and D (bottom), respectively. 
The N-H stretching modes in the region $\mathrm{W}$ show softening similar to $\mathrm{NH}_{3} \mathrm{BH}_{3}$ under pressure where as the spectra from region $\mathrm{D}$ show mixed behavior (both compression and softening of different modes) which also confirms the existence of structurally different phases in that region. The electron density can be tuned with rapidly reducing volumes upon compression [228]. Therefore, pressure-induced chemical reactions are highly possible in borane complexes. Drastic spectral changes observed above $6 \mathrm{GPa}$ in diborane was suggested to be due to enhanced intermolecular interaction with a covalent nature. At higher pressures, as the mobility of the atoms is restricted, the most facilitated phase transformations are the ones which are not associated with large atomic movements. Hence, in a similar way the formation of higher hydrides may be possible in $\mathrm{LiNH}_{2} \mathrm{BH}_{3}$ without drastic changes in the atomic positions at high pressures [228].

\subsection{High P-T investigation of mixture of $\mathrm{MgH}_{2}$ and $\mathrm{AlH}_{3}$}

\subsubsection{Introduction}

Magnesium alanate, $\operatorname{Mg}\left(\mathrm{AlH}_{4}\right)_{2}$, is an interesting complex metal hydride with 9.3 wt $\%$ of hydrogen content. Computational studies suggest that at ambient conditions, $\mathrm{Mg}\left(\mathrm{AlH}_{4}\right)_{2}$ is of trigonal- $\mathrm{P}^{-} 3 \mathrm{~m}_{1}$ structure which transform to a monoclinic- $\mathrm{C} 2 / \mathrm{m}$ structure around $0.68 \mathrm{GPa}$. Above $10.28 \mathrm{GPa}$, transition to an orthorhombic- Pbca structure is expected [77]. The decomposition of $\mathrm{Mg}\left(\mathrm{AlH}_{4}\right)_{2}$ occurs in three steps, first in

the range $110-200{ }^{\circ} \mathrm{C}$, and second and third around $240-380$ and $400{ }^{\circ} \mathrm{C}$, respectively [31]. By the second step, hydrogen is fully released and the third step corresponds to the formation of $\mathrm{Al}_{3} \mathrm{Mg}_{2}$. The enthalpy of reaction for $\mathrm{MgH}_{2}+2 \mathrm{AlH}_{3} \rightarrow \mathrm{Mg}\left(\mathrm{AlH}_{4}\right)_{2}$ is 
calculated in the range 11-29 kJ/mol and for $\mathrm{AlH}_{3}+\mathrm{MgH}_{2} \rightarrow \mathrm{MgAlH}_{5}$ it is $12 \mathrm{~kJ} / \mathrm{mol}$, which suggest that both are endothermic processes which may occur in the same temperature range [231]. In an earlier study, $\mathrm{Mg}\left(\mathrm{AlH}_{4}\right)_{2}$ was synthesized by ball milling the mixture of $\mathrm{AlH}_{3}$ and $\mathrm{MgH}_{2}$ and its decomposition enthalpy was reported as $\sim 0 \mathrm{~kJ} /$ mol $\mathrm{H}_{2}$ [232]. Aim of this study was to synthesize $\mathrm{Mg}\left(\mathrm{AlH}_{4}\right)_{2}$ under high P-T conditions from the stochiometric mixture of $\mathrm{AlH}_{3}$ and $\mathrm{MgH}_{2}$. The sample was heated under pressure up to $150{ }^{\circ} \mathrm{C}$ in steps and the results indicate a reaction has occurred and an unidentified new phase has been formed.

Aluminium hydride is a covalent binary hydride which usually forms polymeric compound $\left(\mathrm{AlH}_{3}\right)_{\mathrm{n}}$ and exist in more than six crystal structures depending on synthesize and processing conditions [158]. $\mathrm{AlH}_{3}$ has a hydrogen content of $10.1 \mathrm{wt} \%$ and decomposes into elements in one step. The decomposition temperature is in the range 60$200{ }^{\circ} \mathrm{C}$ for different polymorphs of $\mathrm{AlH}_{3}$. The hydrogenation of $\mathrm{Al}$ needs about $2.5 \mathrm{GPa}$ of $\mathrm{H}_{2}$ pressure. The $\mathrm{AlH}_{3}$ phases are metastable at room temperature possibly due to the $\mathrm{Al}_{2} \mathrm{O}_{3}$ layer formation. The rhombohedral phase of $\mathrm{AlH}_{3}$ has a reported decomposition temperature of $175^{\circ} \mathrm{C}$, and a $1 \mathrm{hr}$ ball milling reduces it to $125^{\circ} \mathrm{C}$. Sandrock et al. [233], report that controlling particle-size and doping of $\mathrm{AlH}_{3}$ with small levels of alkali-metal hydrides results in accelerated desorption rates. It is found that addition of $20 \mathrm{~mol} \%$ of $\mathrm{LiH}$ reduces the desorption temperature of $\mathrm{AlH}_{3}$ to below $100{ }^{\circ} \mathrm{C}$ which is good enough to supply vehicles. In the present study a rhombohedral- $\mathrm{R}-3 \mathrm{C}$ polymorph $\left(\alpha-\mathrm{AlH}_{3}\right)$ has been used.

High pressure investigations show that $\alpha-\mathrm{AlH}_{3}$ exhibit a monoclinic distortion around $8 \mathrm{GPa}$ and it has a bulk modulus value of $40 \mathrm{GPa}[234,235]$. The reduced cell 
volume at high pressure is accommodated by an octahedral tilting and a decrease of the Al-H bond distance. The formation enthalpy for the rhombohedral phase of $\mathrm{AlH}_{3}$ is reported to be around $-8.2 \mathrm{~kJ} / \mathrm{mol} \mathrm{H}_{2}$ and it has absolute entropy of $20 \mathrm{~J} / \mathrm{K} \mathrm{mol} \mathrm{H}_{2}$. Highpressure experiments have demonstrated that $\mathrm{Al}$ absorbs hydrogen and form aluminum hydride at $2.8 \mathrm{GPa}$ of $\mathrm{H}_{2}$ pressure at $300{ }^{\circ} \mathrm{C}[236]$.

$\mathrm{MgH}_{2}$ contains 7.7 wt $\%$ of hydrogen and the decomposition temperature is more than $325^{\circ} \mathrm{C}$. Several high pressure phases have been observed under $19 \mathrm{GPa}$ for $\mathrm{MgH}_{2}$ which are of tetragonal- $\mathrm{P} 4_{2} / \mathrm{mnm}(0-5.5 \mathrm{GPa})$, orthorhombic- Pben (5.5- $\left.9.5 \mathrm{GPa}\right)$, cubic- $\mathrm{Pa}^{-} 3$ (9.35- 10.36 GPa) and orthorhombic- Pbca (above $10 \mathrm{GPa}$ ) structures [60]. The orthorhombic- Pbcn and tetragonal phases coexist up to $9.5 \mathrm{GPa} . \mathrm{MgH}_{2}$ can be synthesized by low cost means; however its enthalpy of formation is much higher which is around $-76 \mathrm{~kJ} / \mathrm{mol}$ [61]. Equilibrium pressure of $\mathrm{MgH}_{2}$ is found to be about $0.1 \mathrm{MPa}$ at $275{ }^{\circ} \mathrm{C}$ and for a mixture of $\mathrm{MgH}_{2} / \mathrm{Si}$ it is around $10 \mathrm{MPa}$ at $150{ }^{\circ} \mathrm{C}[50,237]$. Thermodynamic properties of $\mathrm{MgH}_{2}$ were computed up to $1273{ }^{\circ} \mathrm{C}$ which indicates an enhanced hydrogen property for this material at high temperature [238]. The bulk modulus for different polymorphs of $\mathrm{MgH}_{2}$ has been determined in the range 44- $50 \mathrm{GPa}$. The computationally determined transition pressures are found to be lower than the experimental values [239]. Among the high pressure phases, $\mathrm{Pbcn}$ structure of $\mathrm{MgH}_{2}$ is stable under ambient conditions owing to its structural similarity with the $\mathrm{P} 4_{2} / \mathrm{mnm}$ system.

Compared to other alkali- earth metal hydrides, $\mathrm{MgH}_{2}$ has more number of phases at high pressures and temperatures. Slow diffusion and kinetics prevent usage of $\mathrm{MgH}_{2}$ as an efficient candidate for hydrogen storage. $\mathrm{MgH}_{2}$ can be synthesized under pressure and 
temperature of $3 \mathrm{MPa}$ and $440{ }^{\circ} \mathrm{C}$, respectively [240]. Bortz et al. [241], studied neutron powder diffraction of $\mathrm{Pbcn}-\mathrm{MgH}_{2}$ formed by heating tetragonal phase to $800{ }^{\circ} \mathrm{C}$ at $2 \mathrm{GPa}$ and quenching to ambient conditions. The reverse transition of orthorhombic to tetragonal phase occurs at $1 \mathrm{~atm}$ and $297^{\circ} \mathrm{C}$. The orthorhombic and tetragonal phases are also found to be coexisting in the pressure ranges $2-8 \mathrm{GPa}$ and $250-900{ }^{\circ} \mathrm{C}$ other than between 5.5 and $9.5 \mathrm{GPa}$ at room temperature [60,241].

\subsubsection{Results and discussions}

\subsubsection{X-ray diffraction}

X-ray diffraction experiments were conducted using a Mo radiation of $\lambda=$ $0.71073 \AA$ and synchrotron source with $\lambda=0.495937 \AA$. The loaded DAC was heated $e x$ situ in an oven. Figure 11.6 shows micro XRD patterns collected using a synchrotron source at room temperature after heating the sample at different pressures. The XRD pattern collected at ambient conditions shows the sample is a homogeneous mixture of $\mathrm{MgH}_{2}$ and $\mathrm{AlH}_{3}$. After heating at $110{ }^{\circ} \mathrm{C}$ and $0.7 \mathrm{GPa}$ for more than $12 \mathrm{hrs}$, the XRD patterns show $\mathrm{MgH}_{2}$ and $\mathrm{Al}$. This imply decomposition of $\mathrm{AlH}_{3}$ at this pressure and temperature. When pressure was increased to $3.4 \mathrm{GPa}$, a shoulder was observed in the $\mathrm{Al}$ peak which may be due to a partial reaction of $\mathrm{Al}$ with $\mathrm{MgH}_{2}$ and $\mathrm{H}_{2}$. Pure $\mathrm{Al}$ metal do not show any phase transition up to $27 \mathrm{GPa}[242]$. After increasing pressure to $7.2 \mathrm{GPa}$, the sample was again heated at $150{ }^{\circ} \mathrm{C}$ for about $12 \mathrm{hrs}$ and found new peaks in the XRD pattern which was stable on decompression. Therefore we may conclude that some of the new peaks observed after heating at 7.2 GPa is due to orthorhombic- Pbcn phase which coexist with tetragonal- $\mathrm{MgH}_{2}$ up to $9.5 \mathrm{GPa}$. The highest pressure achieved in the 
present experiment was $\sim 9 \mathrm{GPa}$ which is below the complete transition pressure of $\mathrm{MgH}_{2}$. Therefore, even after release of pressure both $\mathrm{MgH}_{2}$ phases were present along with $\mathrm{Al}$ and an unidentified phase. The experimental results show that $\operatorname{Mg}\left(\mathrm{AlH}_{4}\right)_{2}$ formation did not occur as expected.

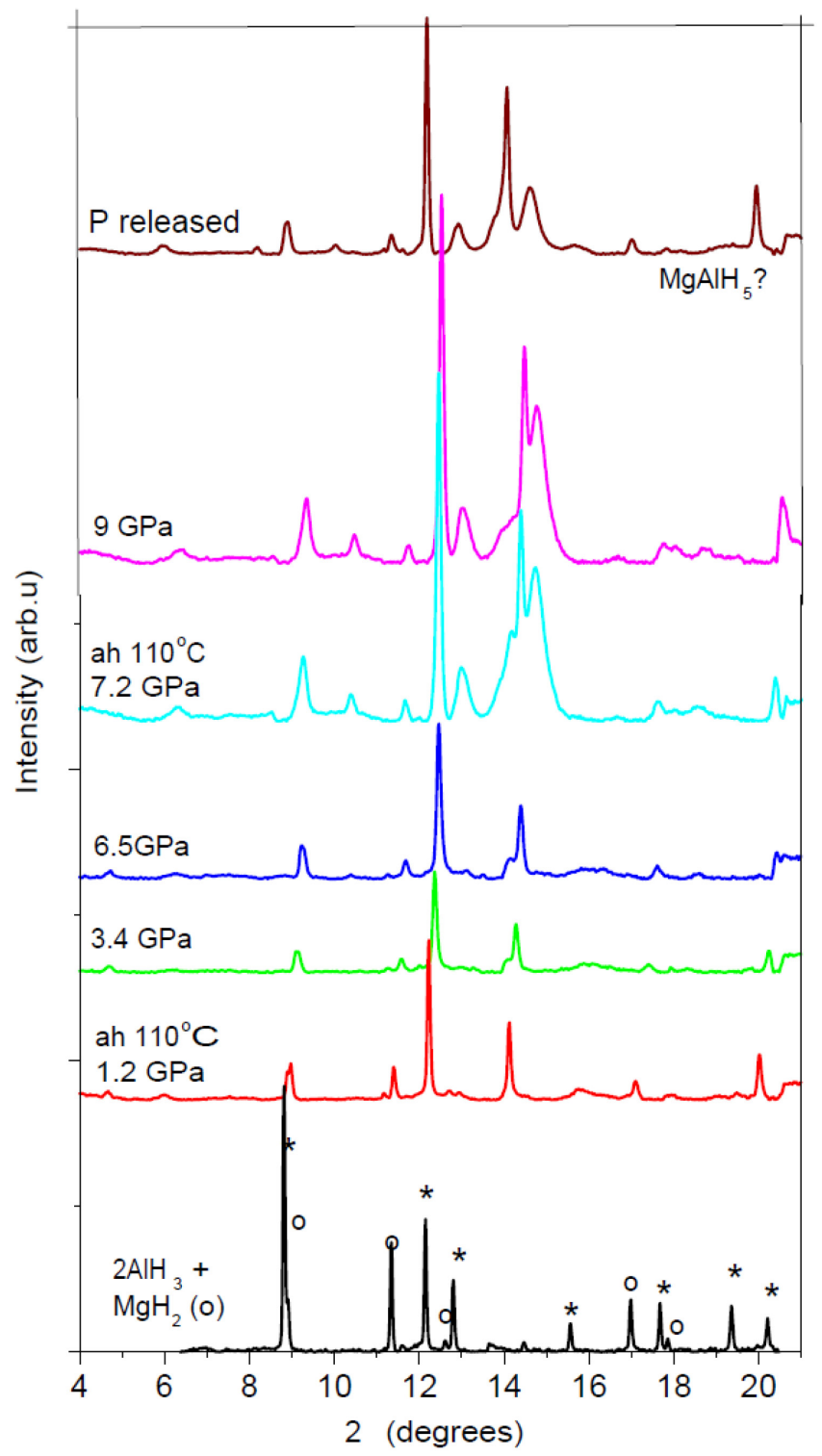

Figure 11. 6: The micro XRD patterns of mixture of $\mathrm{AlH}_{3}$ and $\mathrm{MgH}_{2}$ collected at various pressures and room temperature. Abbreviations: bh- before heating, ah- after heating. 
Figure 11.7 shows there are two unidentified peaks at $2 \theta \sim 13^{\circ}$ and $\sim 14.5^{\circ}$ which may corresponds to a new phase. Appearance of a new peak was also observed in Raman spectroscopic study $\sim 3.4 \mathrm{GPa}$ suggesting a partial reaction between $\mathrm{MgH}_{2}, \mathrm{Al}$ and $\mathrm{H}_{2}$ around this pressure. The expected reaction of $\mathrm{Al}$ and $\mathrm{H}_{2}$ was not occurred above 2.8 GPa which may be due to the low partial pressure of hydrogen in the sample chamber.

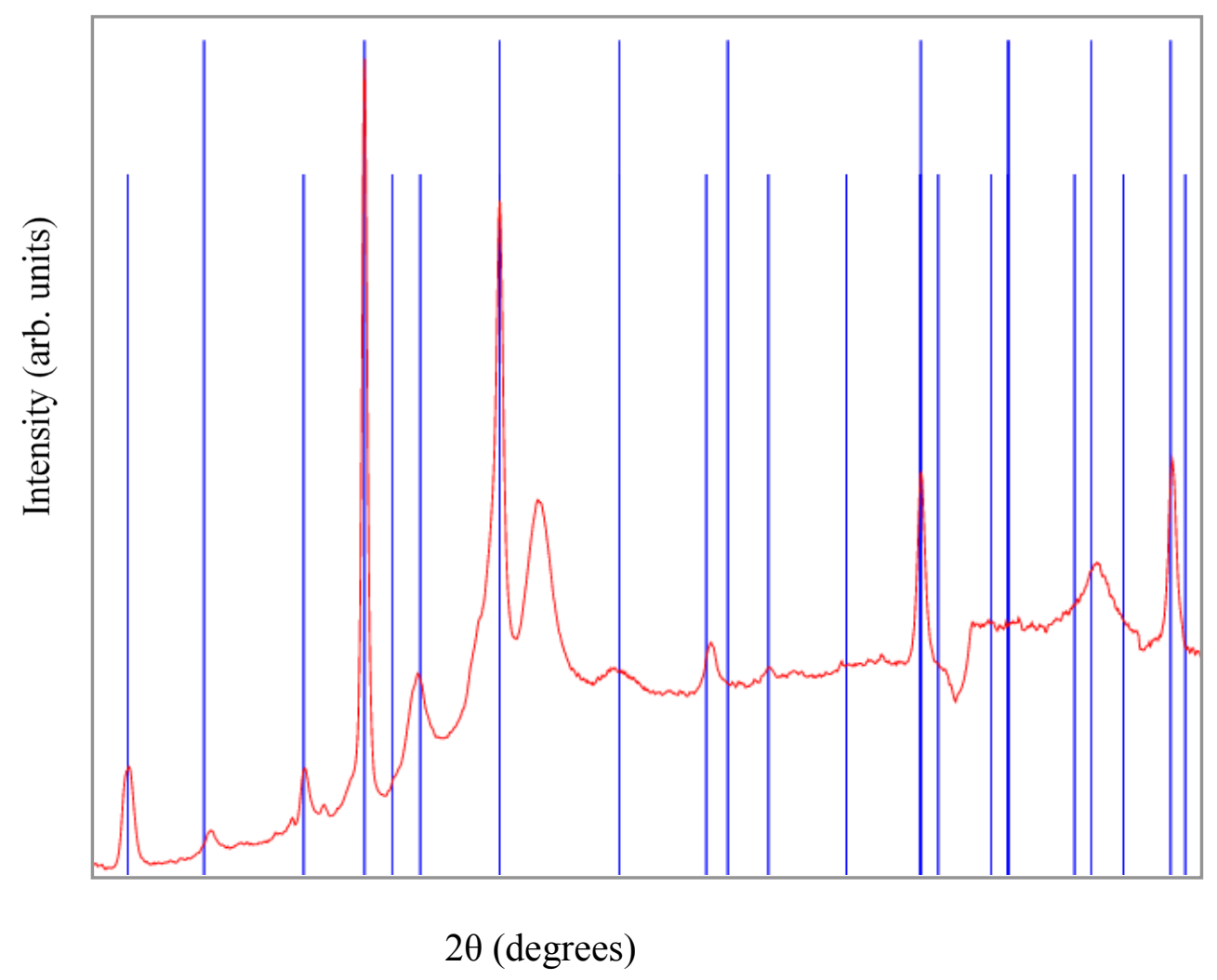

Figure 11. 7: The XRD pattern from decompressed sample at room temperature after heating under pressure up to $9 \mathrm{GPa}$ matched with peaks corresponds to $\mathrm{MgH}_{2}$ (shorter lines) and $\mathrm{Al}$ (longer lines).

Rietveld analysis of the obtained XRD pattern shows that the sample, after high P-T treatment, is not a single phase like $\mathrm{MgAlH}_{5}$, but a mixture of two or three phases. 
$\mathrm{MgAlH}_{5}$ is a possible decomposition product of magnesium alanate in the temperature range $120-155{ }^{\circ} \mathrm{C}$ by the reaction: $2 \mathrm{Mg}\left(\mathrm{AlH}_{4}\right)_{2} \rightarrow 2 \mathrm{MgAlH}_{5}+2 \mathrm{Al}+3 \mathrm{H}_{2}$ [243]. In summary, a partial reaction of the components is found to be occurred and a M-Al-H system is formed.

High pressure XRD patterns of $\mathrm{Mg}\left(\mathrm{AlH}_{4}\right)_{2}$ with $\mathrm{LiCl}$ (marked*) impurities are shown in Figure 11.8. The micro XRD experiments were conducted with X-rays of wavelength $\sim 0.4151 \AA$. At ambient conditions $\mathrm{Mg}\left(\mathrm{AlH}_{4}\right)_{2}$ is of trigonal- $\mathrm{P}^{-} 3 \mathrm{~m}_{1}$ structure. At $0.68 \mathrm{GPa}$ it transform to a monoclinic- $\mathrm{C} 2 / \mathrm{m}$ structure. It agrees with theoretical calculations of $\mathrm{Hu}$ et al. [77]. Above $10.28 \mathrm{GPa}$ transition to an orthorhombic- Pbca structure is expected but was not observed in this study up to $13 \mathrm{GPa}$.

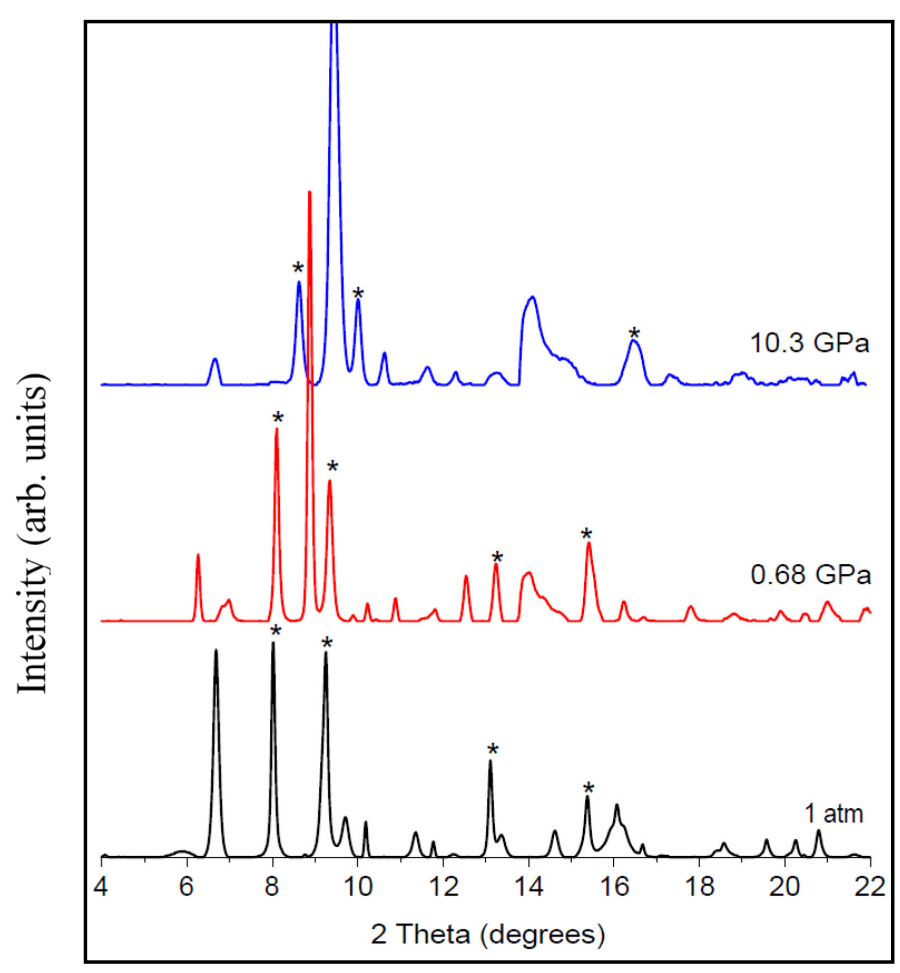

Figure 11. 8: XRD patterns of $\mathrm{Mg}\left(\mathrm{AlH}_{4}\right)_{2}$ containing $\mathrm{LiCl}$ (marked *) at some selected pressures. 


\subsubsection{Raman spectroscopy}

The mixture of $2 \mathrm{AlH}_{3}+\mathrm{MgH}_{2}$ was heated at $\sim 110{ }^{\circ} \mathrm{C}$ and $0.1 \mathrm{MPa}$ for $12 \mathrm{hrs}$. After heating $\mathrm{AlH}_{3}$ was decomposed to $\mathrm{Al}$ and $\mathrm{H}_{2}$. The mixture was then cooled to room temperature and compressed in steps of pressure. A new peak was emerged in the Raman spectra at $3.5 \mathrm{GPa}$ which is marked with arrow in Figure 11.9. This resembles the Al-H bending mode at 766 and $821 \mathrm{~cm}^{-1}$ of $\operatorname{Mg}\left(\mathrm{AlH}_{4}\right)_{2}$ and hence the reaction of $\mathrm{Al}$ with $\mathrm{MgH}_{2}$ or $\mathrm{H}_{2}$ can be expected.

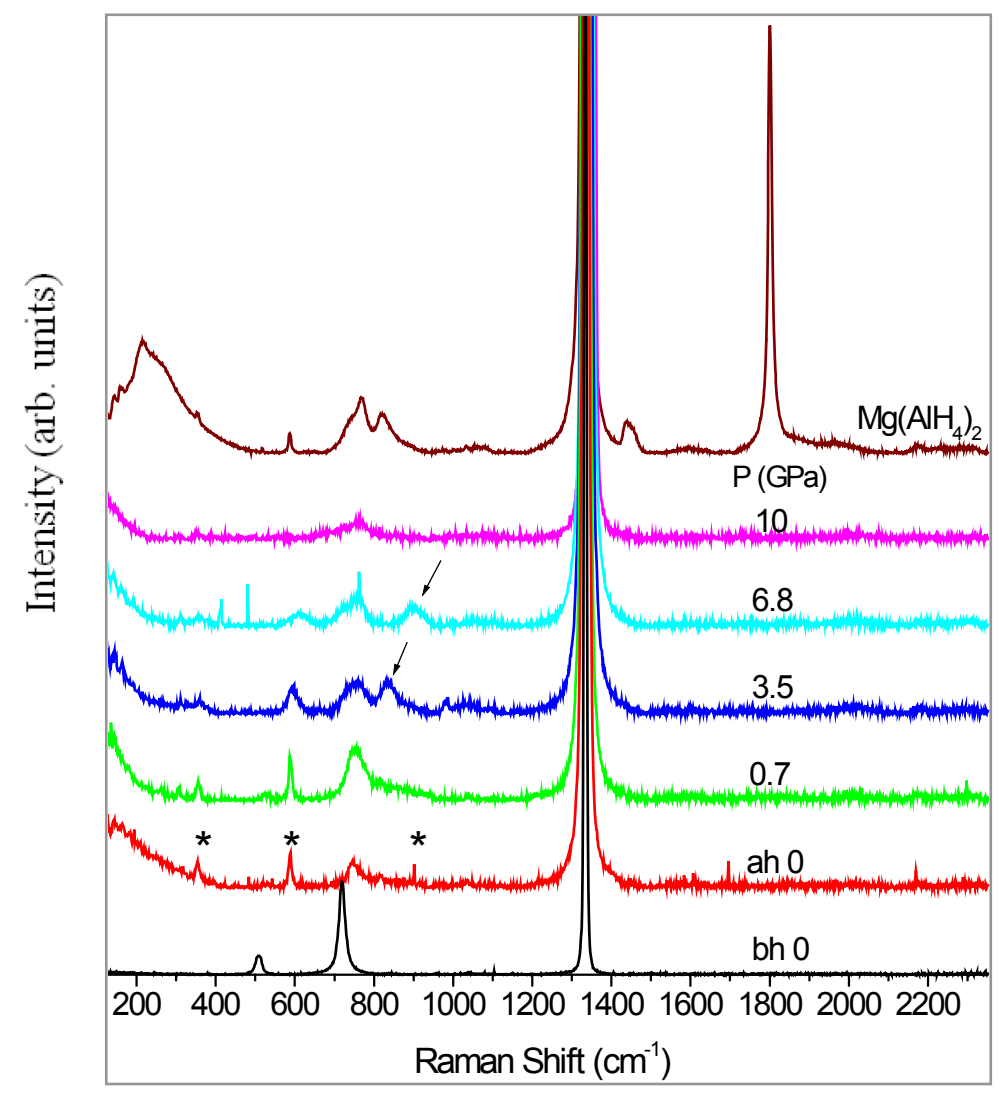

Figure 11. 9: Raman spectra of the $2 \mathrm{AlH}_{3}+\mathrm{MgH}_{2}$ mixture collected at various pressures in comparison with the Raman spectra of $\operatorname{Mg}\left(\mathrm{AlH}_{4}\right)_{2}$. 
The pressure was gradually increased up to $10 \mathrm{GPa}$. The new peak was disappeared at $10 \mathrm{GPa}$ and was reappeared on decompression below $4 \mathrm{GPa}$. Which implies that the new peak is not due to $\mathrm{MgH}_{2}$ as the orthorhombic phase is stable on decompression confirming a possible reaction of $\mathrm{Al}$ with $\mathrm{MgH}_{2}$ or $\mathrm{H}_{2}$. While, in the XRD pattern at $10 \mathrm{GPa}$, the new peaks were not disappeared and was stable on decompression. Therefore, it may be expected that the disappearence of peaks in the Raman spectra above $10 \mathrm{GPa}$ can be due to freezing of vibrations other than amorphization or decomposition. Figure 11.10 shows Raman spectra of pure $\operatorname{Mg}\left(\mathrm{AlH}_{4}\right)_{2}$ at various pressures.

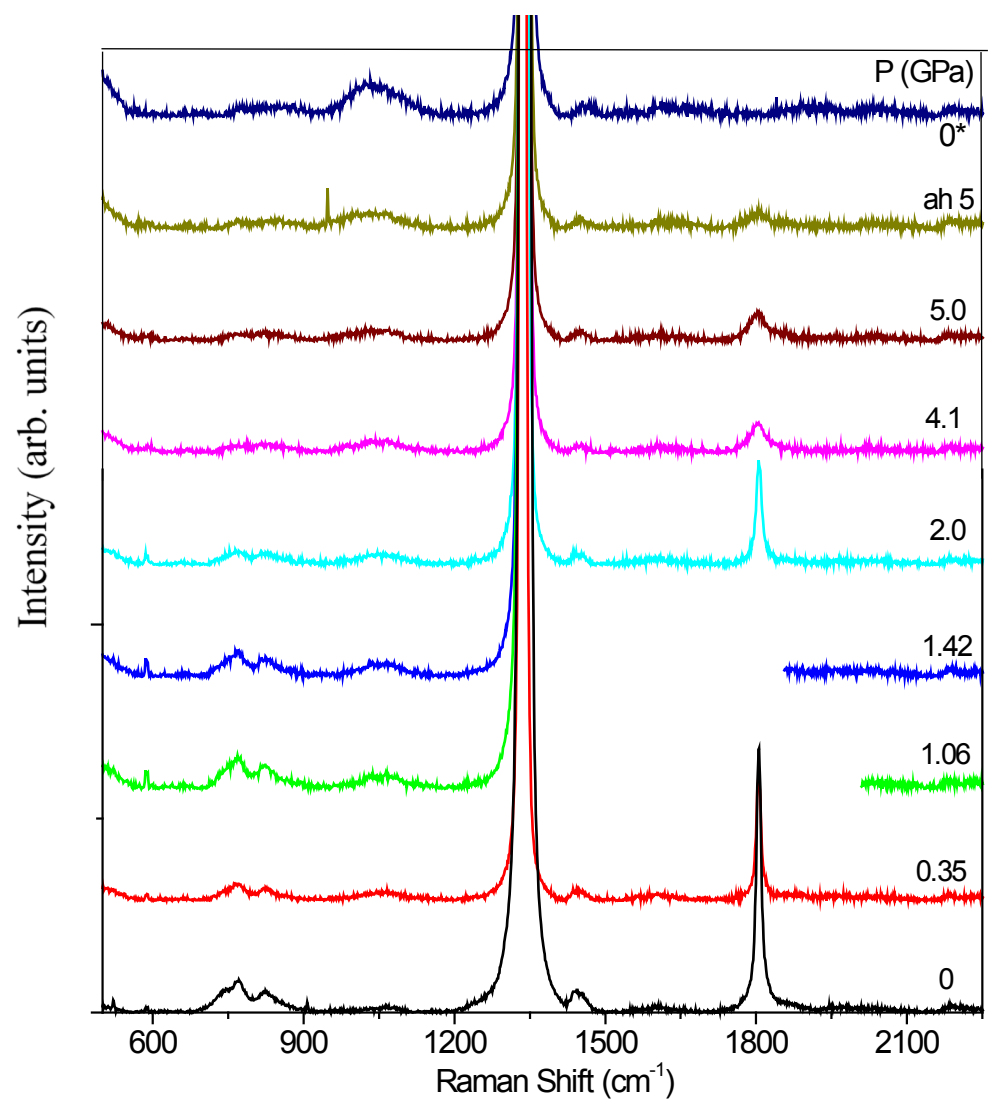

Figure 11. 10: Raman spectra of $\mathrm{Mg}\left(\mathrm{AlH}_{4}\right)_{2}$ collected at various pressures. Spectrum marked $*$ is of the decompressed sample and 'ah' abbreviates after heating. 
In the high pressure study of pure $\mathrm{Mg}\left(\mathrm{AlH}_{4}\right)_{2}$, small amount of $\mathrm{H}_{2}$ was released above $0.35 \mathrm{GPa}$. This may be caused by the reaction of $\mathrm{Mg}\left(\mathrm{AlH}_{4}\right)_{2}$ with moisture on the gasket hole surface. When pressure was increased above $5 \mathrm{GPa}$, the strong $\mathrm{Al}-\mathrm{H}$ stretching mode $\sim 1800 \mathrm{~cm}^{-1}$ disappeared and this transition was irreversible. Raman spectroscopic studies of $\mathrm{LiAlH}_{4}$ and $\mathrm{NaAlH}_{4}$ also show disappearance of Al-H stretching modes at high pressures. To check effect of stress field, the sample cell was heated at 150 ${ }^{\circ} \mathrm{C}$ for about 6 hrs at $5 \mathrm{GPa}$ (spectrum marked ah in Figure 11. 10) and found no change.

\subsection{Mixtures of Si and $\mathrm{MgH}_{2}$}

\subsubsection{Introduction}

$\mathrm{MgH}_{2}$ contains $7.7 \mathrm{wt} \%$ of hydrogen and the decomposition temperature is above $325{ }^{\circ} \mathrm{C}$. Thermodynamic properties of $\mathrm{MgH}_{2}$ were computed up to $1273{ }^{\circ} \mathrm{C}$ which indicates an enhanced hydrogen storage property for this material at high temperatures [238]. The slow diffusion and kinetics prevent usage of $\mathrm{MgH}_{2}$ as an efficient candidate for hydrogen storage. Hence it is important to identify the best additive for destabilizing this light metal hydride. $\mathrm{MgH}_{2}$ can be synthesized by low cost means; however its enthalpy of formation is much higher, around $-76 \mathrm{~kJ} / \mathrm{mol}$ [61]. Equilibrium pressure of $\mathrm{MgH}_{2}$ at $275{ }^{\circ} \mathrm{C}$ is about $0.1 \mathrm{MPa}$ and for a mixture of $\mathrm{MgH}_{2}$ and $\mathrm{Si}$ it is about $10 \mathrm{MPa}$ at $150{ }^{\circ} \mathrm{C}[50,237]$. This result indicates that the $\mathrm{MgH}_{2} / \mathrm{Si}$ system, which has a hydrogen capacity of $5 \mathrm{wt} \%$, could be applicable for hydrogen storage at reduced temperatures. The $\mathrm{MgH}_{2} / \mathrm{Si}$ system was found to be not readily reversible and the hydrogenation of the $\mathrm{Mg}_{2} \mathrm{Si}$ appears to be kinetically limited. 
If a compound is stable with respect to its constituent elements, then the hydride will be effectively destabilized. This occurs because the system can be cycled between the hydride and the compound instead of the elemental metals and $\mathrm{H}_{2}$. For pure $\mathrm{MgH}_{2}$, hydrogen desorption begins at approximately $295{ }^{\circ} \mathrm{C}$, and an equilibrium pressure of 0.17 MPa was attained at $300{ }^{\circ} \mathrm{C}[50]$. After milling the mixture of $\mathrm{MgH}_{2}$ and $\mathrm{Si}$ and thermal dehydrogenation up to $400{ }^{\circ} \mathrm{C}, \mathrm{Mg}_{2} \mathrm{Si}$ was the predominant phase, with a small amount of residual $\mathrm{Si}$ [50]. The reaction can be represented as: $2 \mathrm{MgH}_{2}+\mathrm{Si} \rightarrow \mathrm{Mg}_{2} \mathrm{Si}+2 \mathrm{H}_{2}$. The standard enthalpy of formation of $\mathrm{Mg}_{2} \mathrm{Si}$ is $-77.8 \mathrm{~kJ} / \mathrm{mol}$ [50]. Therefore, formation of $\mathrm{Mg}_{2} \mathrm{Si}$ reduces the standard enthalpy of dehydrogenation, which is $\sim 75.3 \mathrm{~kJ} / \mathrm{mol}$ for pure $\mathrm{MgH}_{2}$ to $36.4 \mathrm{~kJ} / \mathrm{mol}$ for $\mathrm{MgH}_{2}+1 / 2 \mathrm{Si}$. High temperature studies of $\mathrm{Mg}_{2} \mathrm{Si}$ up to $700{ }^{\circ} \mathrm{C}$ show it is stable in that temperature ranges [244]. The present study aim to synthesize a compound by the reaction: $3 \mathrm{MgH}_{2}+\mathrm{Si} \rightarrow \mathrm{MgSiH}_{6}+2 \mathrm{Mg}$.

\subsubsection{Results and discussion}

The high pressure experiments at room temperature on the mixture of $\mathrm{Si}$ and $\mathrm{MgH}_{2}$ showed only phase transitions of $\mathrm{MgH}_{2}$. Figure 11.11 shows XRD patterns of the mixture and the components Si and $\mathrm{MgH}_{2}$ collected at using X-rays of $\lambda=0.71073 \AA$. When the sample mixture was heated at $3.8 \mathrm{GPa}$ and $600{ }^{\circ} \mathrm{C}$ formation $\mathrm{Mg}$ and $\mathrm{H}_{2}$ implying the decomposition of $\mathrm{MgH}_{2}$ without reacting to $\mathrm{Si}$. In another experiment, the mixture of $\mathrm{MgH}_{2}$ and $\mathrm{Si}$ was heated at $0.5 \mathrm{GPa}$ and $600{ }^{\circ} \mathrm{C}$ with a fresh loading using a zirconia anvil cell. After cooling down the cell, Raman spectra shows formation of $\mathrm{Mg}_{2} \mathrm{Si}$ alloy. Further studies has to be conducted to estimate the $\mathrm{H}_{2}$ pressure required to rehydrogenate $\mathrm{Mg}_{2} \mathrm{Si}$. The observed reaction is: $3 \mathrm{MgH}_{2}+\mathrm{Si} \rightarrow \mathrm{Mg}_{2} \mathrm{Si}+\mathrm{MgH}_{2}+2 \mathrm{H}_{2}$. 


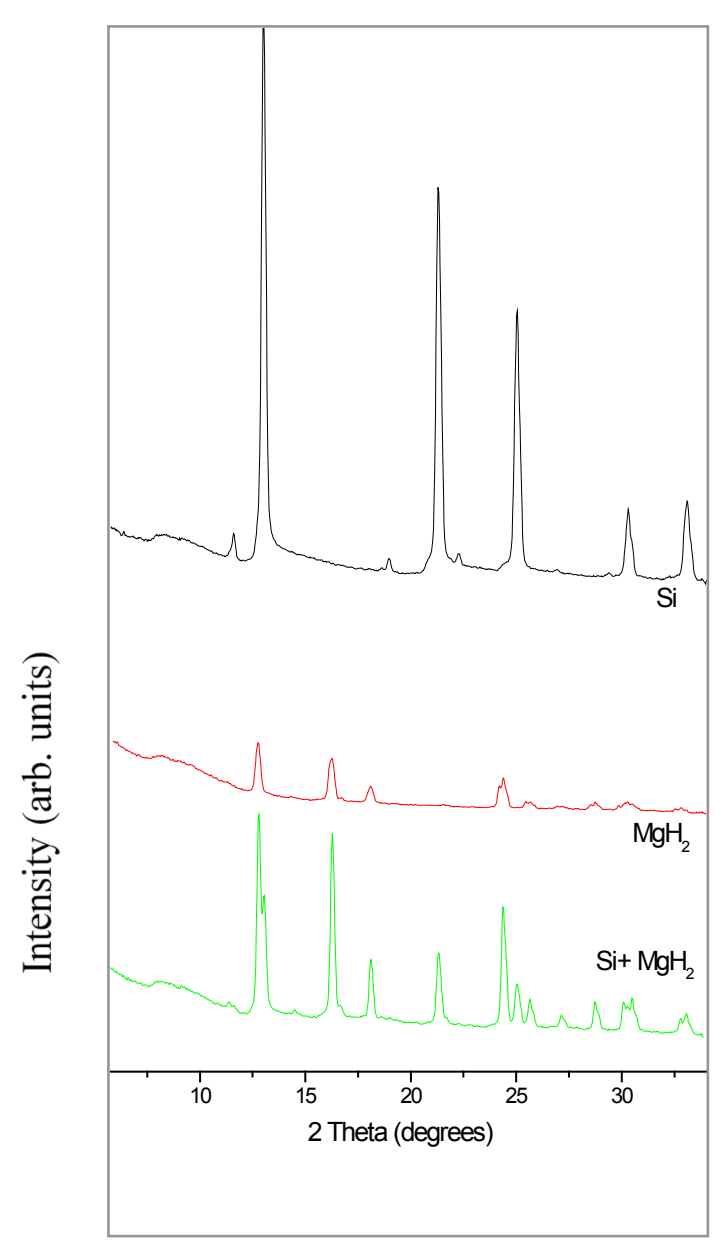

(a)

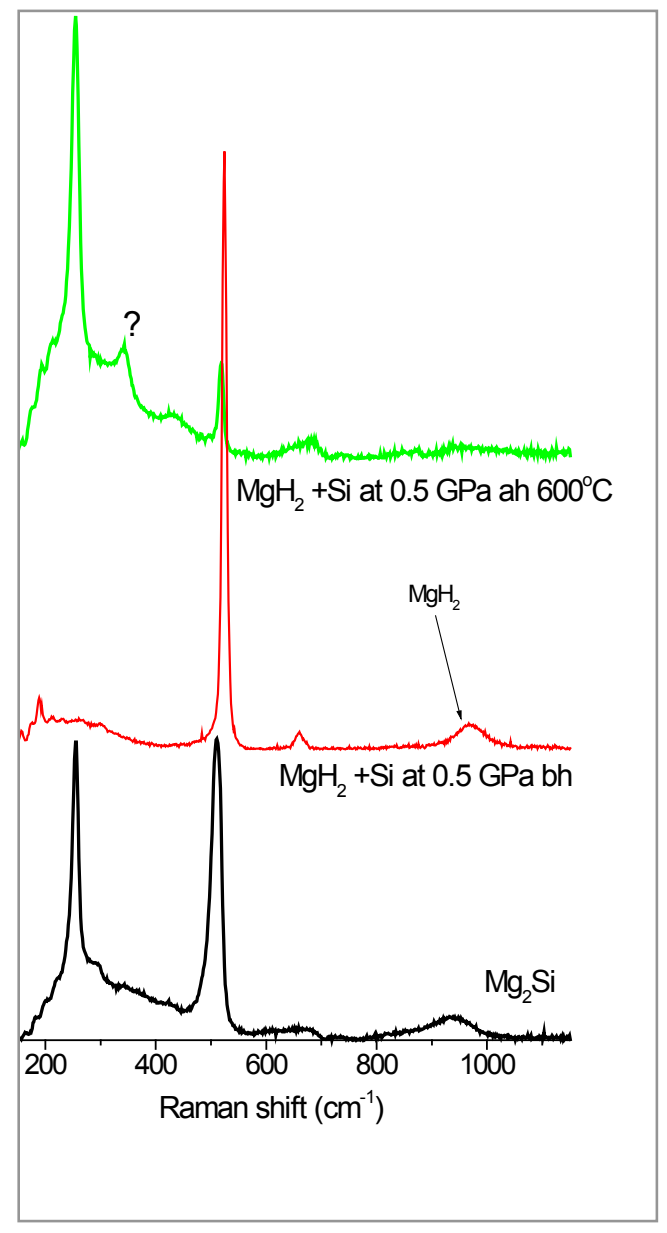

(b)

Figure 11. 11: (a) The XRD pattern of the mixture of $\mathrm{Si}$ and $\mathrm{MgH}_{2}$ in comparison to that of pure components. (b) Raman spectra of $\mathrm{MgH}_{2}+\mathrm{Si}$ mixture before and after heating at $0.5 \mathrm{GPa}$ and $600{ }^{\circ} \mathrm{C}$ and that of pure $\mathrm{Mg}_{2} \mathrm{Si}$; bh and ah abbreviates before and after heating, respectively. 


\section{CHAPTER 12. COMPARISON OF PROPERTIES OF HYDRIDES}

\subsection{Properties of Metals and Hydrides: A Comparison}

Estimation of different thermodynamic properties is required to determine the stability of a hydride for suitable technological applications. In general, hydride formation is accompanied by a structural phase transformation of the metal which has effect on their properties. Therefore, it is important to correlate properties of both metal and metal hydride to understand how the hydrogen influences the material properties. Table 12.1 shows approximate value of molar volume of hydrogen atom in the boron hydrides. The molar volume of boron hydrides are calculated from their unit cell volume. The molar volume of B metal is $\sim 4.5 \mathrm{~cm}^{3} / \mathrm{mol}$. The difference of the molar volumes of the boron hydride and boron atoms gives an approximate value of the volume occupied by the hydrogen atoms. Average increase in molar volume per $\mathrm{H}$ - atom in the boron hydrides is found to be $\sim 6 \mathrm{~cm}^{3} / \mathrm{mol}$. The values of thermodynamic properties of metal may increase or decrease when it form a hydride. Table 12.2 summarizes structure and entropy change due to hydride formation of some metal hydrides. Decrease or increase of entropy may be a combined effect of structural transformation and charge transfer between the metal and H- atom. 
Table 12. 1: Approximate values of molar volumes of hydrogen atoms in boron hydrides.

\begin{tabular}{llll}
\hline $\begin{array}{c}\text { Hydride } \\
\mathrm{B}_{\mathrm{x}} \mathrm{H}_{\mathbf{y}}[245]\end{array}$ & $\begin{array}{l}\mathbf{V}_{\text {mol }} \\
\left(\mathrm{cm}^{3} / \mathrm{mol}\right)\end{array}$ & $\begin{array}{l}\sum \mathbf{V}_{\text {mol }} \mathbf{B} \\
\left(\mathrm{cm}^{3} / \mathrm{mol}\right)\end{array}$ & $\begin{array}{l}\boldsymbol{\Delta \mathbf { V } _ { \text { mol } } \text { of } \mathrm { H }} \\
\text { in } \mathrm{B}_{\mathrm{x}} \mathrm{H}_{\mathbf{y}}\end{array}$ \\
\hline $\mathrm{B}_{10} \mathrm{H}_{14}$ & 123.41 & 45.6 & 5.56 \\
$\mathrm{~B}_{10} \mathrm{H}_{16}$ & 145.83 & 45.6 & 6.26 \\
$\mathrm{~B}_{12} \mathrm{H}_{16}$ & 158.47 & 54.7 & 6.48 \\
$\mathrm{~B}_{13} \mathrm{H}_{19}$ & 176.08 & 59.3 & 6.15 \\
$\mathrm{~B}_{14} \mathrm{H}_{20}$ & 176.54 & 63.9 & 5.63 \\
$\mathrm{~B}_{16} \mathrm{H}_{20}$ & 197.91 & 73 & 6.24 \\
$\mathrm{~B}_{18} \mathrm{H}_{22}$ & 215.21 & 82.1 & 6.05 \\
$\mathrm{~B}_{18} \mathrm{H}_{23}$ & 213.41 & 82.1 & 5.71 \\
$\mathrm{~B}_{20} \mathrm{H}_{26}$ & 246.52 & 91.2 & 5.97 \\
$\mathrm{~B}_{2} \mathrm{H}_{6}$ & 46.65 & 9.13 & 6.25 \\
\hline $\mathrm{BH}_{3}$ & 23.6 & 4.5 & 6.37 \\
$\mathrm{~B}_{4} \mathrm{H}_{10}$ & 73.60 & 18.3 & 5.53 \\
\hline $\mathrm{B}_{5} \mathrm{H}_{11}$ & 87.44 & 22.8 & 5.87 \\
$\mathrm{~B}_{5} \mathrm{H}_{9}$ & 78.26 & 22.8 & 6.16 \\
\hline $\mathrm{B}_{6} \mathrm{H}_{10}$ & 88.50 & 27.4 & 6.11 \\
$\mathrm{~B}_{8} \mathrm{H}_{12}$ & 110.99 & 36.5 & 6.21 \\
\hline
\end{tabular}

Table 12. 2: Variation of the entropy of hydrogen in some binary metal hydrides $\left(\mathrm{MH}_{\mathrm{x}}\right)$.

\begin{tabular}{llll}
\hline $\mathrm{MH}_{\mathrm{x}}$ & $\begin{array}{c}\mathrm{MH}_{\mathrm{x}} \\
\text { structure }\end{array}$ & $\begin{array}{c}\mathrm{M} \\
\text { structure }\end{array}$ & $\Delta \mathrm{S}$ \\
\hline $\mathrm{PrH}_{2}$ & fcc- $\mathrm{Fm}^{-} 3 \mathrm{~m}$ & hcp- $\mathrm{P}_{3} / \mathrm{mmc}$ & -8.58 \\
$\mathrm{NdH}_{2}$ & fcc $-\mathrm{Fm}^{-} 3 \mathrm{~m}$ & hcp- $\mathrm{P}_{3} / \mathrm{mmc}$ & -6.09 \\
$\mathrm{LaH}_{2}$ & fcc $-\mathrm{Fm}^{-} 3 \mathrm{~m}$ & hcp- $\mathrm{P}_{3} / \mathrm{mmc}$ & -2.61 \\
$\mathrm{ZrH}_{2}$ & fcc $-\mathrm{Fm}^{-} 3 \mathrm{~m}$ & hcp $-\mathrm{P}_{3} / \mathrm{mmc}$ & -1.92 \\
$\mathrm{YH}_{2}$ & fcc $-\mathrm{Fm}^{-} 3 \mathrm{~m}$ & hcp $-\mathrm{P}_{3} / \mathrm{mmc}$ & -3.03 \\
$\mathrm{TiH}_{2}$ & fcc $-\mathrm{Fm}^{-} 3 \mathrm{~m}$ & hcp $-\mathrm{P} 6_{3} / \mathrm{mmc}$ & -0.52 \\
$\mathrm{LiH}$ & fcc $-\mathrm{Fm}^{-} 3 \mathrm{~m}$ & bcc $-\mathrm{Im} 3 \mathrm{~m}$ & -9.05 \\
$\mathrm{CsH}$ & $\mathrm{fcc}-\mathrm{Fm}^{-} 3 \mathrm{~m}$ & bcc $-\mathrm{Im} 3 \mathrm{~m}$ & 0.24 \\
$\mathrm{RbH}$ & fcc $-\mathrm{Fm}^{-} 3 \mathrm{~m}$ & bcc $-\mathrm{Im} 3 \mathrm{~m}$ & 8.62 \\
$\mathrm{KH}$ & fcc $-\mathrm{Fm}^{-} 3 \mathrm{~m}$ & bcc $-\mathrm{Im} 3 \mathrm{~m}$ & -14.46 \\
$\mathrm{NaH}$ & fcc $-\mathrm{Fm}^{-} 3 \mathrm{~m}$ & bcc $-\mathrm{Im} 3 \mathrm{~m}$ & -11.44 \\
\hline
\end{tabular}


A detailed hydride research database is required for proper retrieval of information already available. In this study, data of various properties of more than 950 hydrides were collected from previous publications and public databases. The dataset thus generated is an efficient tool to disseminate the material properties for hydrogen storage technology. The aim of this work is to design new, better hydrides by combining or interpreting the already available data. The hydride database has enabled the study of inter-relationships of hydride material properties such as entropy, molar volume, density, unit cell volume etc., with those of corresponding metals or metal alloys. Figures 12.112.6 show comparative study of various properties of hydrides and the constituent metals.

Alkali metal hydrides have less molar volume than the corresponding metals. The entropy-molar volume relationship (Figure 12.3) of hydrides is found to be different from that of host metals. Analyzing the Figure 12.3, entropy of a hydride can be related to its molar volume by the simple formula $\mathrm{S}_{\mathrm{MH}}=-3.074+2.153 \mathrm{~V}_{\mathrm{MH}}$. Molar volume of a hydride can be represented as $\mathrm{V}_{\mathrm{MH}}=\mathrm{V}_{\mathrm{M}^{+}}+\mathrm{V}_{\mathrm{H}^{+}} \Delta$, where $\mathrm{V}_{\mathrm{M}}$ is the molar volume of metal/alloy, $\mathrm{V}_{\mathrm{H}}$ is the molar volume of hydrogen as a metal $\left(\sim 1.7 \mathrm{~cm}^{3} / \mathrm{mol}\right), \Delta$ is molar volume due to charge transfer between metal and hydrogen atoms. There may be an influence of crystal structure on the value of $\Delta$ and hence molar volume of hydride [246]. Larger the difference between electro-negativities of metal and hydrogen, greater will be the charge transfer effects and hence the change in molar volume. The density of the alkali metal hydride is higher than the corresponding metals may be caused by the crystal structure difference. The alkali- hydrides have fcc (face centered cubic) metal lattice with a packing density of 0.74 compared to 0.68 for corresponding bcc (body centered cubic) metal lattice. 


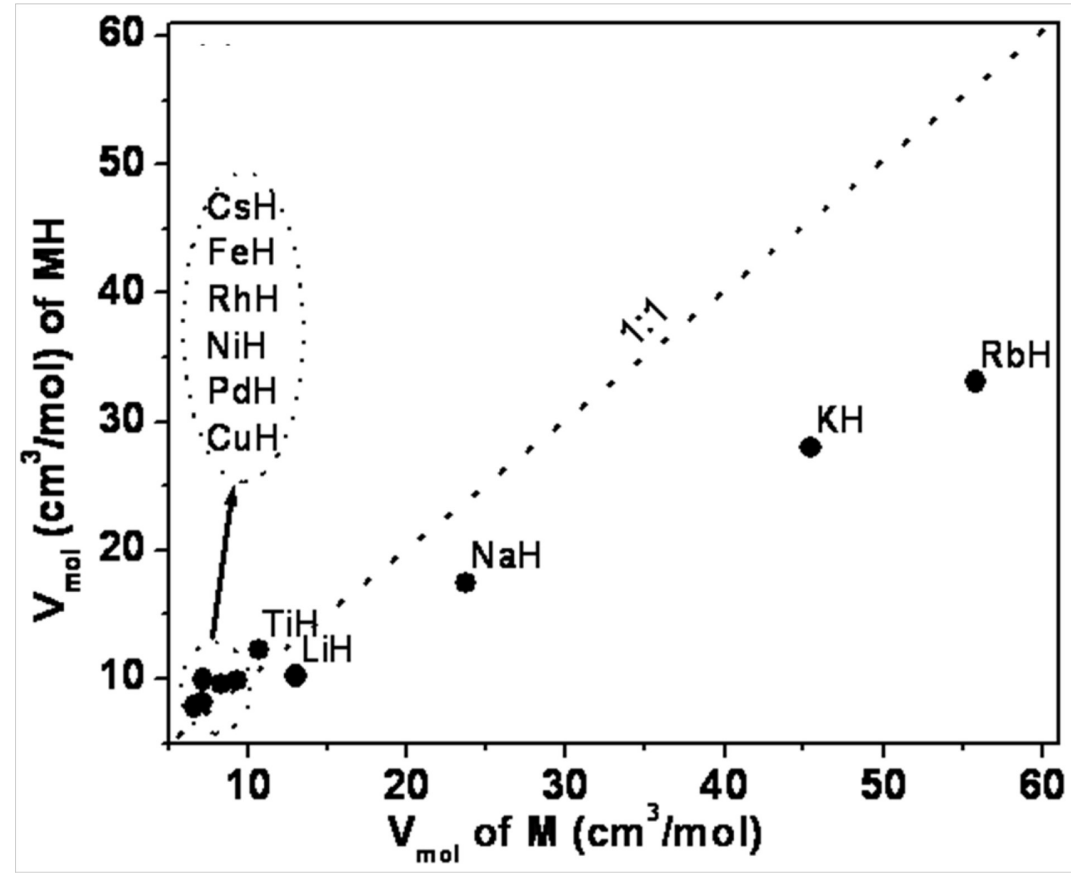

Figure 12. 1: Comparison of molar volume of binary hydrides and corresponding metal.

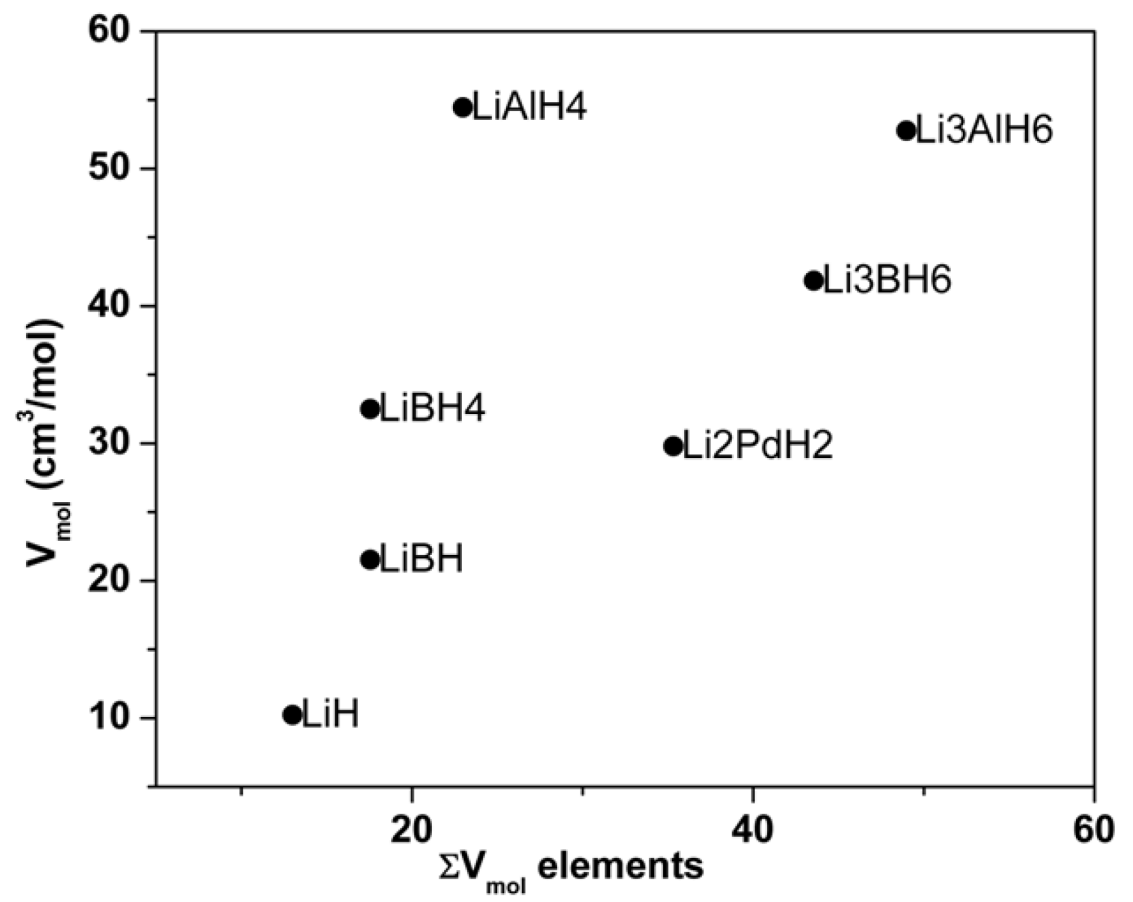

Figure 12. 2: Molar volume of Li containing hydrides versus the element sum [70, 71, $73,83,170,247-249]$. 


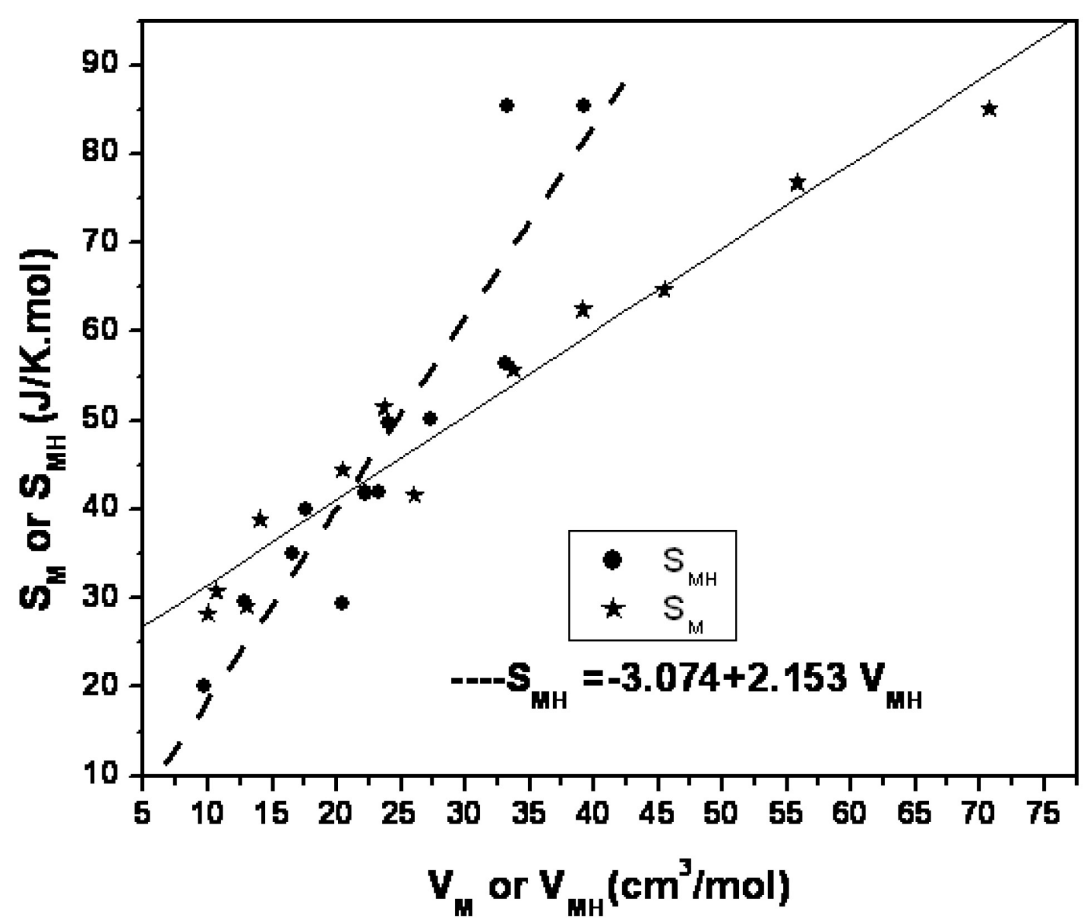

Figure 12. 3: A comparison of entropy versus molar volume of metals and metal hydrides. Subscript M denotes metal and MH denotes metal hydride.

In summary, the increase or decrease of entropy, density and volume can be a combined effect of structural transformation and charge transfer between metal and $\mathrm{H}$ atom during the hydride formation. From Figure 12.5 it is obvious that hydrogenating a metal results in large increase of its specific heat which may be due to hydrogen induced optical vibration [250]. The hydrides which contain elements of same group or period also show some similarity in their properties. Plot of molar volume of various alkali metal hydrides versus the sum of that of host metals is shown in Figure 12.6. This plot suggests that there may be an interrelationship between crystal chemistry and hydrogen induced properties. Hydrides having similar symmetry should show interrelationship among their properties which may vary from those of different symmetry. 


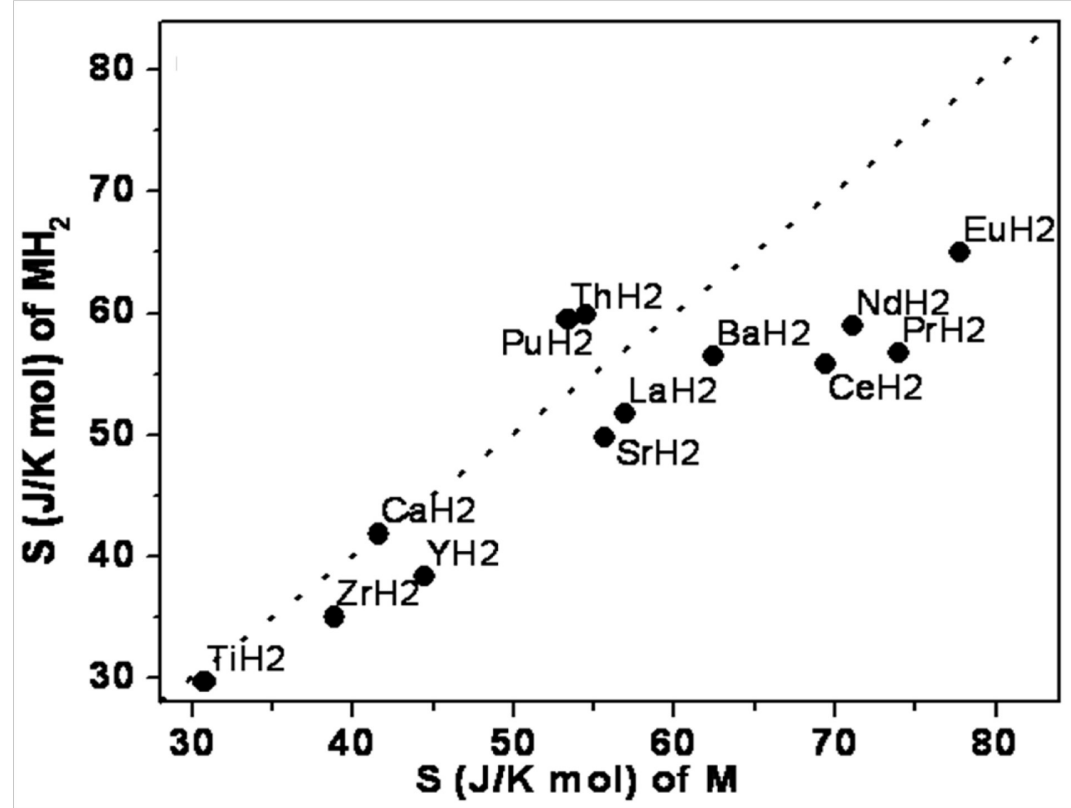

Figure 12. 4: Plots of entropy of binary metal hydrides versus that of corresponding metals.

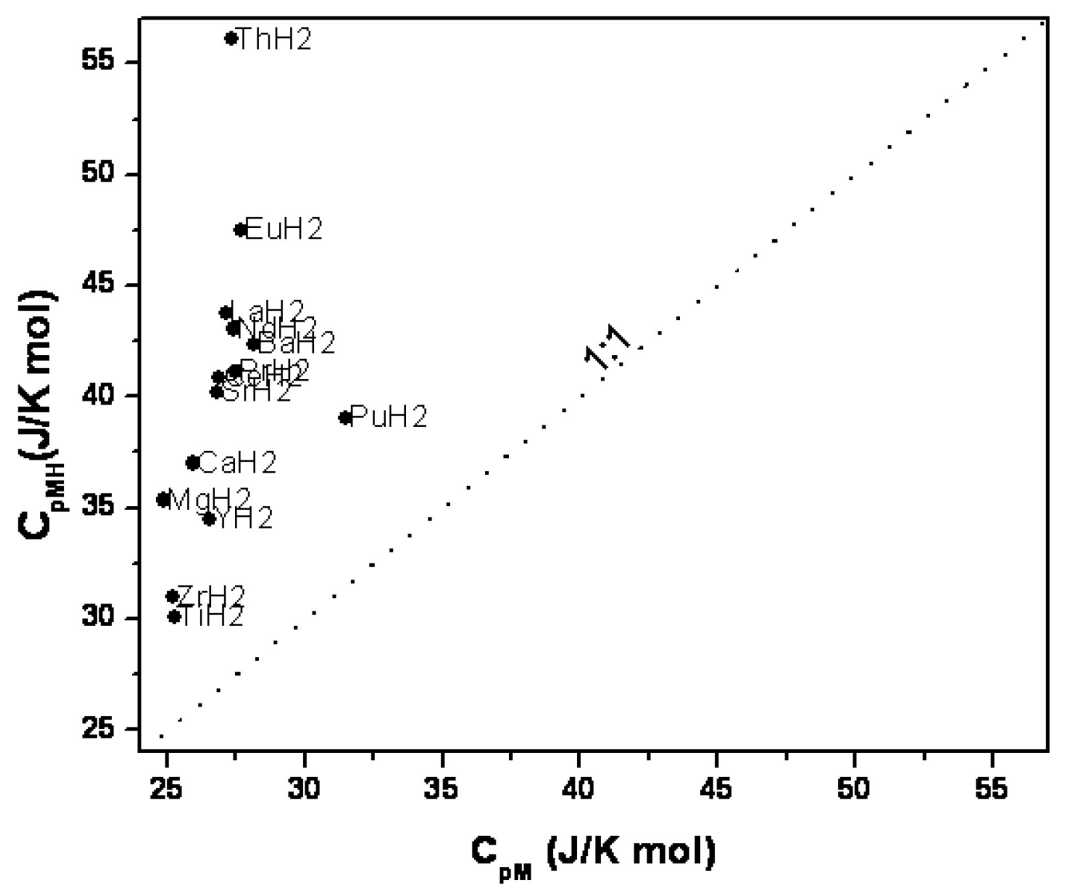

Figure 12. 5: Plot of specific heat of various binary metal hydrides versus that of the host metal. The dotted line corresponds to the specific heat of metals. 


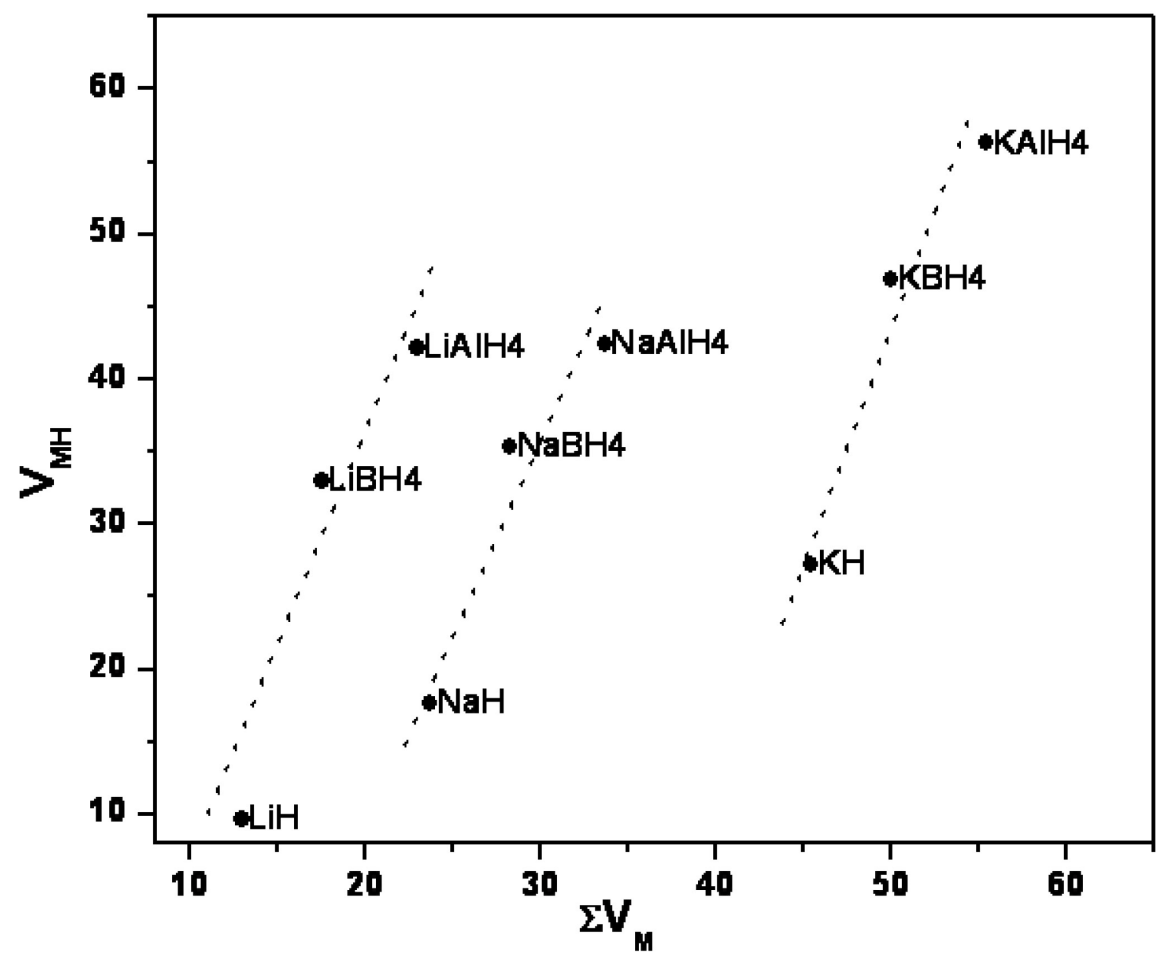

Figure 12. 6: A comparison of molar volumes $\left(\mathrm{cm}^{3} / \mathrm{mol}\right)$ of various alkali hydrides with that of the corresponding host metals.

\subsection{Inter-relationships of Thermodynamic Properties of Hydrides}

Purpose of this study is to explore the thermodynamic properties of metal hydrides which have no established relationships. This type of studies can give insight into the influence of atomic or structural properties on the thermodynamics of the hydrides. Figures 12.7- 12.11 show the interrelation of entropy (taken from FactSage database), hydrogen content, specific heat, enthalpy and bulk modulus to molar volume of metal hydrides. The enthalpy of alkali hydrides is found to be increasing with atomic number, where as it is decreasing in the case of alkali earth metal hydrides. This can be due to large number of $\mathrm{H}-\mathrm{H}$ bonds in the alkali- earth metal hydrides or because of the 
partially ionic nature of $\mathrm{M}-\mathrm{H}$ bonds. The entropy is found to be increasing in both cases with increase in atomic number while $\mathrm{CsH}$ and $\mathrm{BeH}_{2}$ show some deviation. This may be attributed to covalent nature of $\mathrm{BeH}_{2}$ while others are ionic. The large size of Cs and the orintational freedom of hydrogen atoms may be causing a partially ionic bonding of $\mathrm{CsH}$. This in turn can result in the deviation of properties of $\mathrm{CsH}$ from that of similar hydrides.

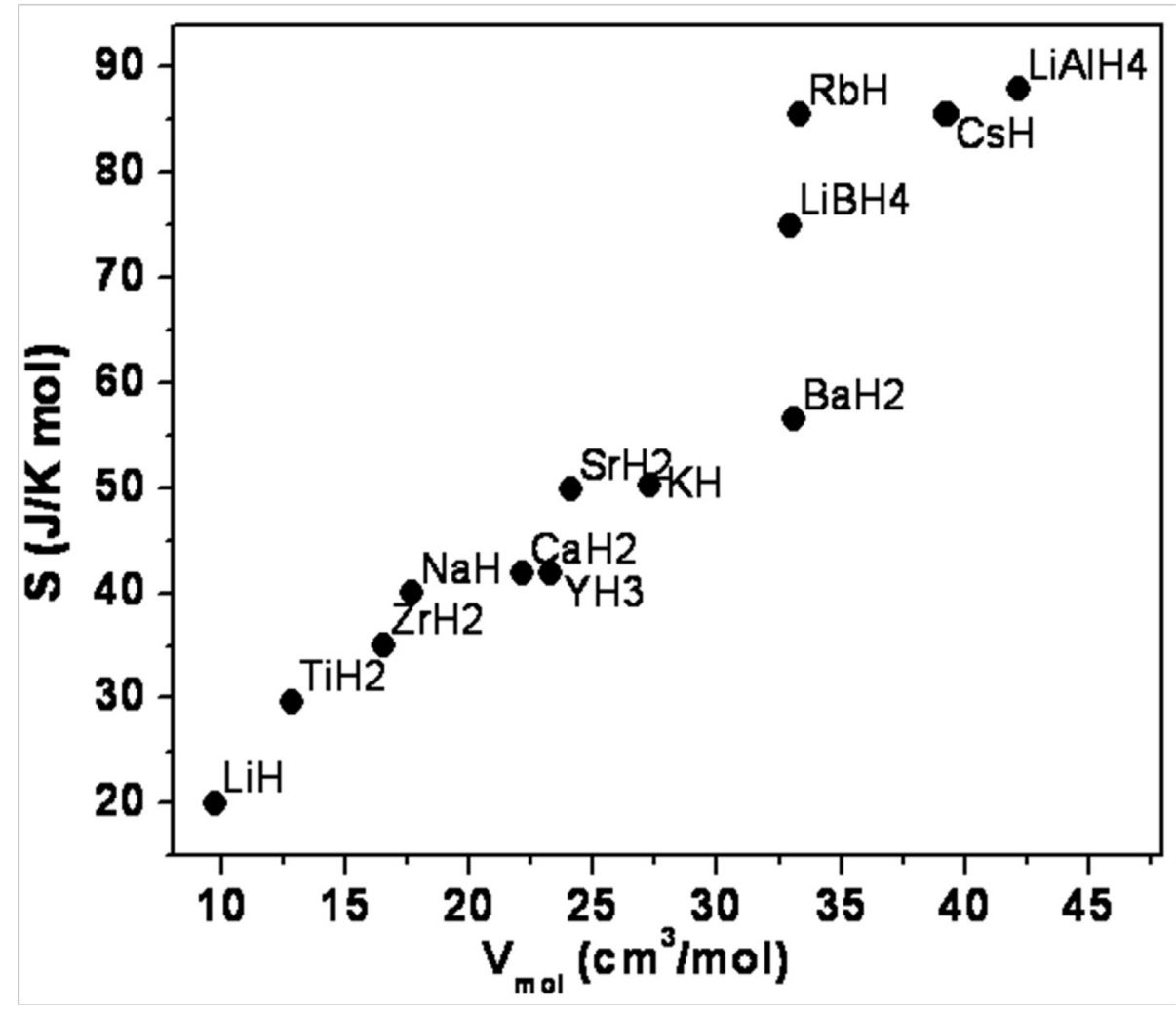

Figure 12. 7: Variation of entropy with molar volume of various hydrides [62, 249-253]. 


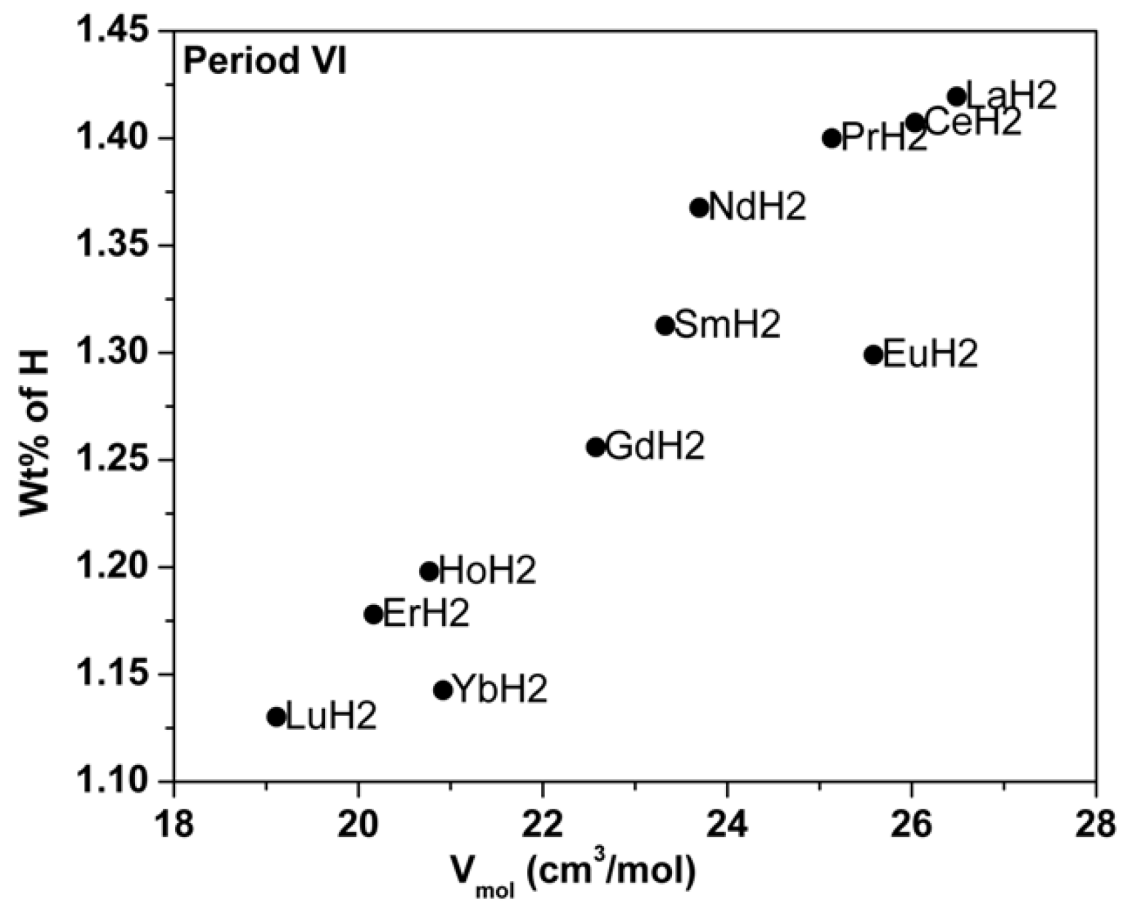

Figure 12. 8: The variation of hydrogen content versus molar volume for the rare-earth metal hydrides.

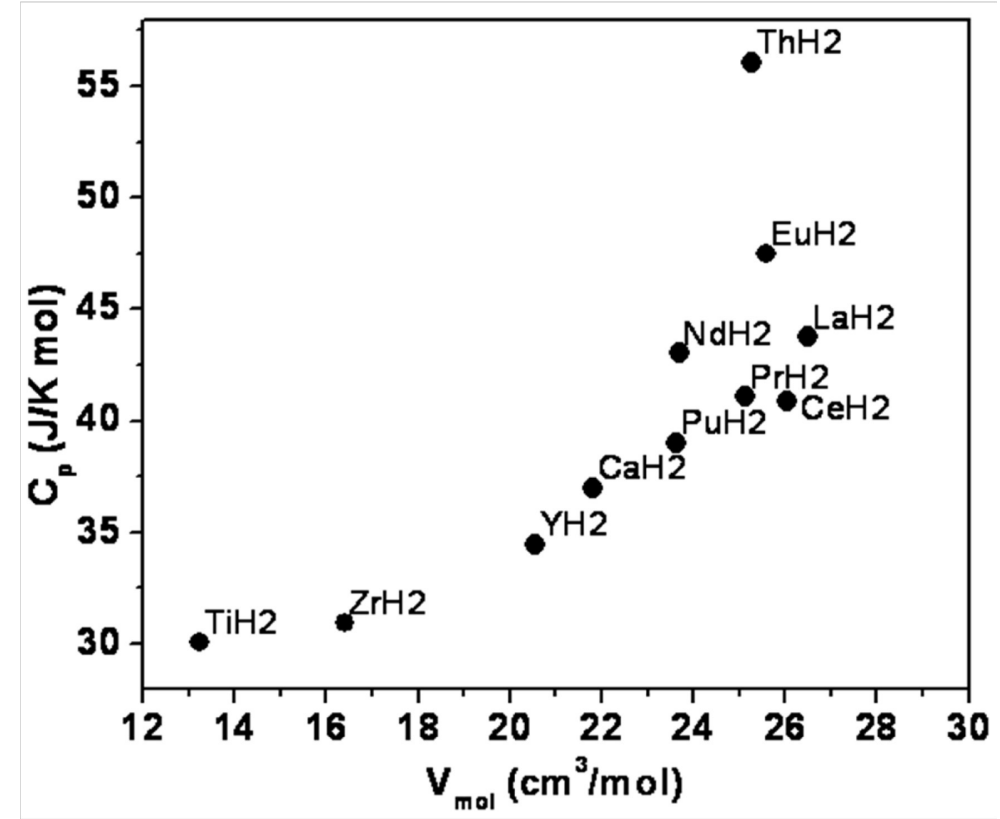

Figure 12. 9: The variation of specific heat versus molar volume for binary metal hydrides $[70,71,73,83,170]$. 
From the plot of variation of bulk modulus with molar volume (Figure 12.11) of metal hydrides, it can be observed that heavy alkali hydrides are more compressible than the alkaline earth metal hydrides. The high compressibility of heavy alkali metal hydrides suggests that tuning of electron density is possible in these compounds under pressure. Therefore, chemical reaction with additives should be investigated for these hydrides as a step towards their destabilization. There is some disparity in the data of $\mathrm{BaH}_{2}$ as it has a lower value of bulk modulus (experimental: $24 \mathrm{GPa}$, calculated: $31 \mathrm{GPa}$ ) as compared to similar hydrides. This deviation from behavior of other alkali- earth metal hydrides implies that the high pressure study of $\mathrm{BaH}_{2}$ has to be redone to confirm the accuracy of the data.

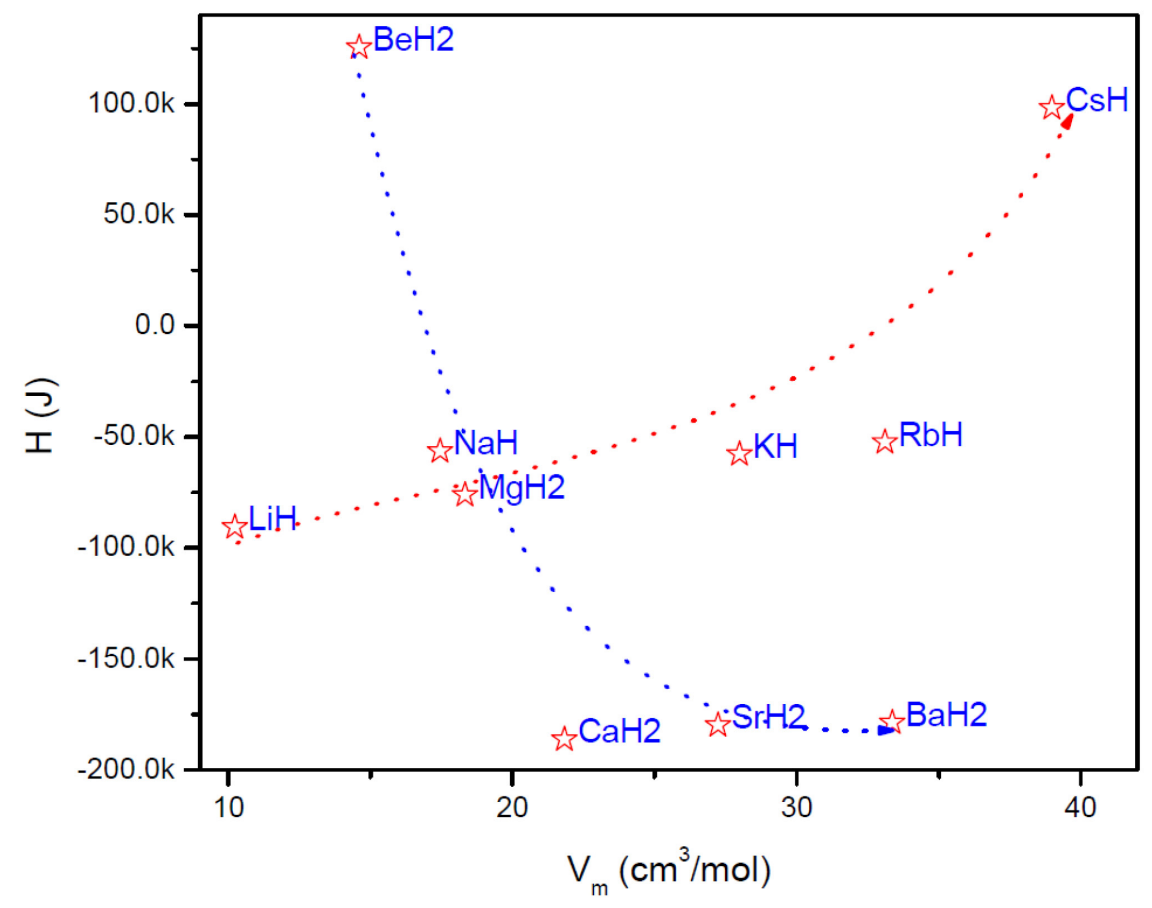

Figure 12. 10: Plot of enthalpy versus molar volume of alkali and alkali- earth metal hydrides. 


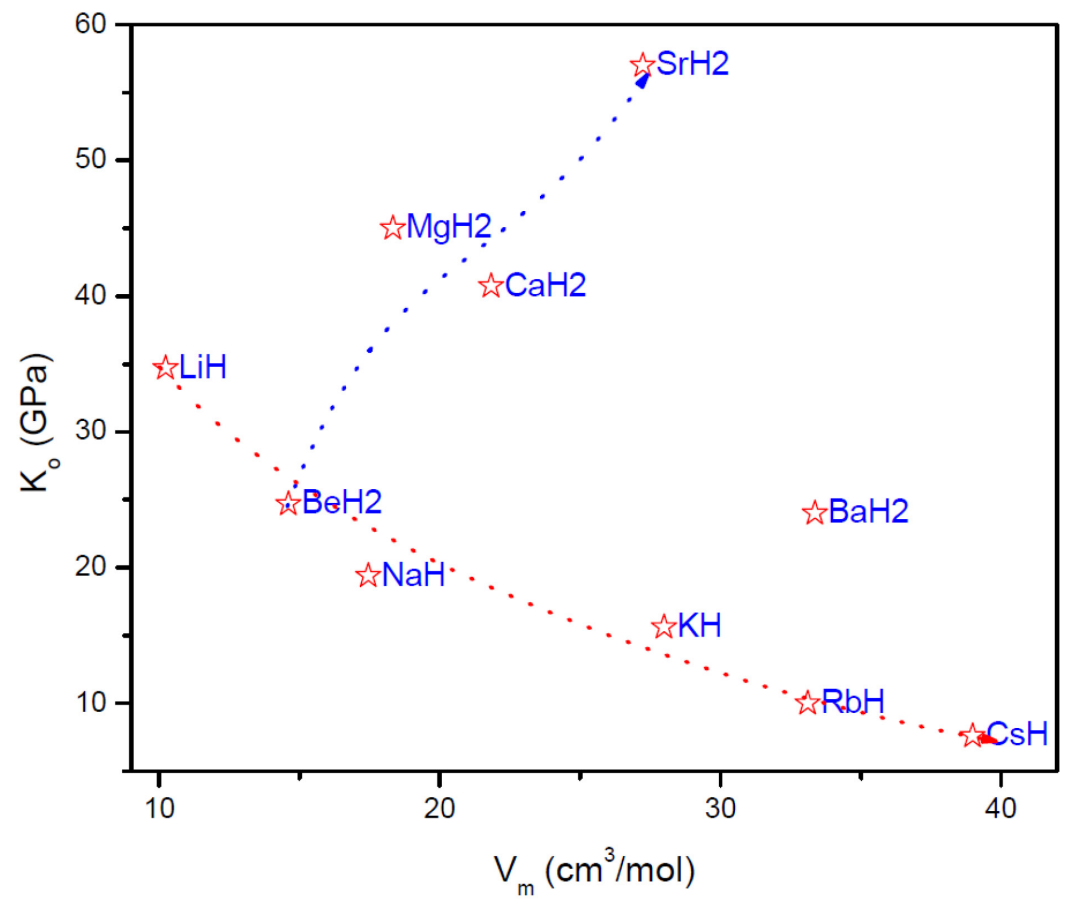

Figure 12. 11: Plot of bulk modulus versus molar volume of alkali and alkali- earth metal hydrides.

Figure 12.12 shows plot of decomposition temperature versus density of alkali and alkali- earth metal hydrides. High density alkali hydrides decompose at lower temperatures than the low density compounds. The alkali- earth metal hydrides show opposite behavior. This may be caused by the difference in hydrogen bonding in those hydrides. 


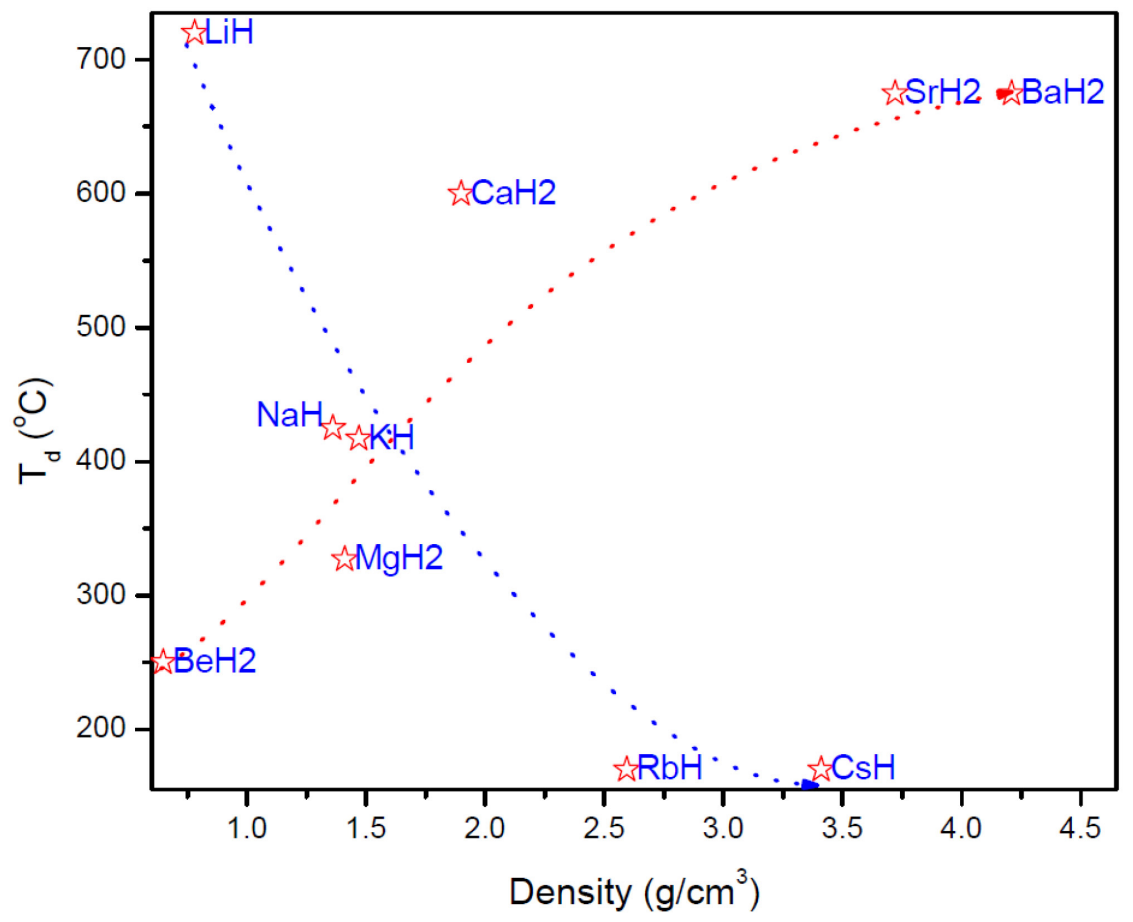

Figure 12. 12: Plot of decomposition temperature versus density of alkali and alkaliearth metal hydrides.

Alanates are less stable than corresponding borohydrides because the Al-H bonds are weaker than the B-H bonds. The decomposition temperature rises as size of the metal increases from Li to $\mathrm{Cs}$ for alkali borohydrides (Figure 12.13). Plot of bulk modulus versus molar volume of alkali and alkali- earth complex hydrides (Figure 12.14) show some similarity between light borohydrides and heavy alanates and vice versa. The bulk modulus values indicate that complex hydrides are more compressible than metals and metal hydrides. Therefore, it will be interesting to study their destabilization by additives/catalysts. This is possible by tuning the electron density of these complex hydrides under pressure. 


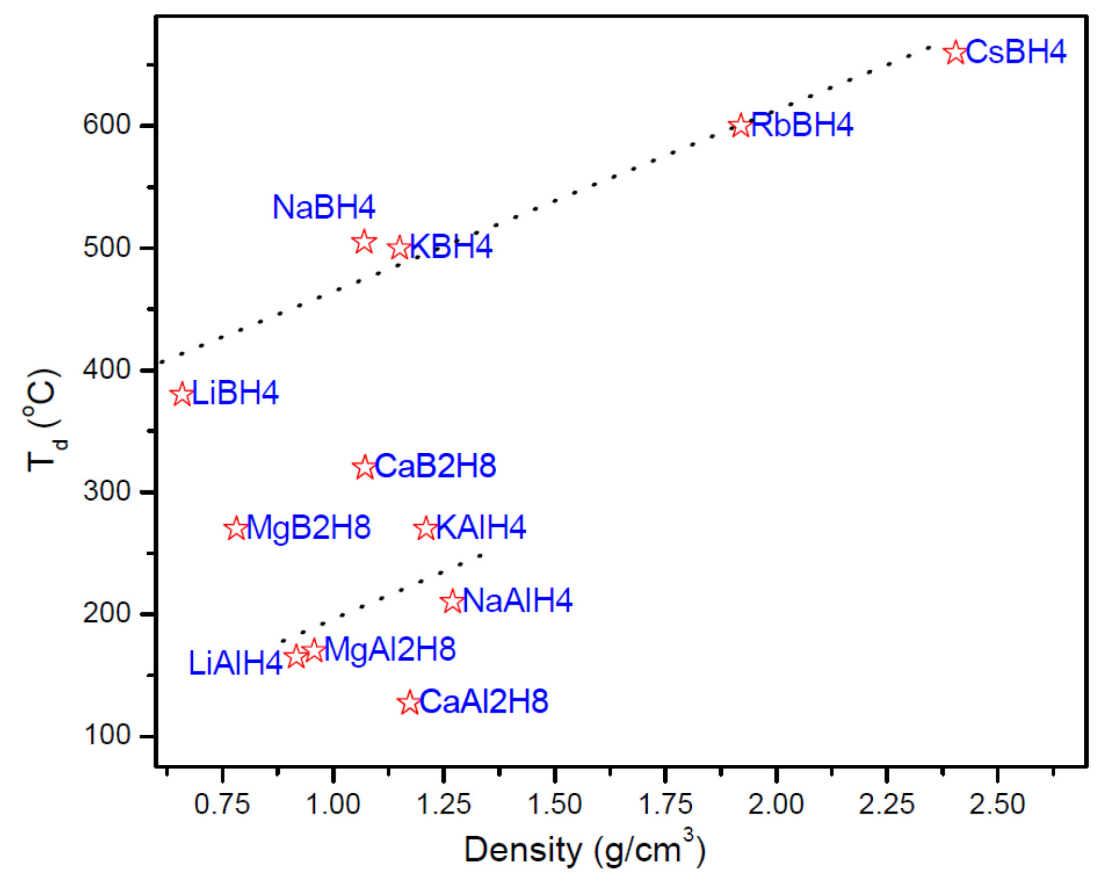

Figure 12. 13: Plot of decomposition temperature versus density of alkali and alkaliearth complex hydrides.

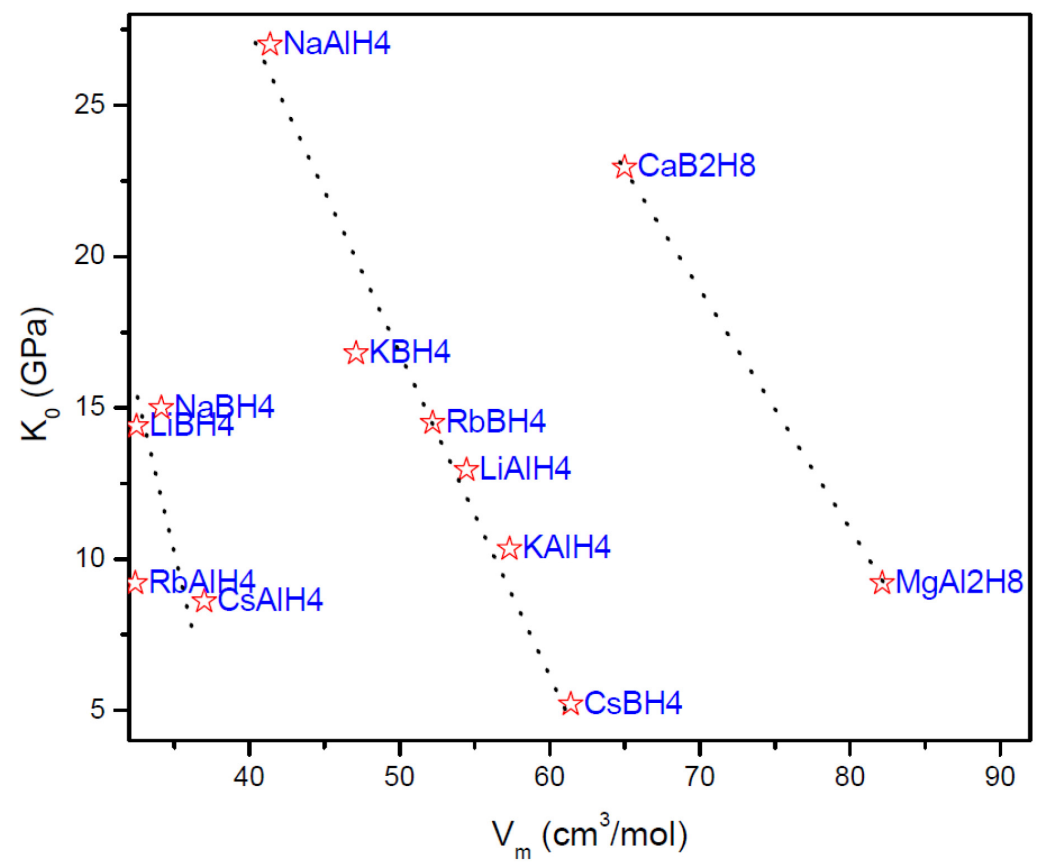

Figure 12. 14: Plot of bulk modulus versus molar volume of alkali and alkali- earth complex hydrides. 


\subsection{Structural Properties of Complex Metal Hydrides}

The high pressure structural behavior of alkali and alkali- earth metal borohydrides is widely investigated. High pressure phase transitions of alkali metal borohydrides are found to be first-order and fully reversible whereas that of alkali- earth complex hydrides are irreversible. Due to strong covalent and ionic bonding nature, dissociation temperatures of borohydrides are very high. The improvement of the hydride properties by catalytic addition requires better understanding of the crystal structure and stability of the phases. The ambient phase of $\mathrm{LiBH}_{4}$ which has an orthorhombic- Pnma structure transforms initially into a tetragonal- $\mathrm{A} m a 2$ at $1.2 \mathrm{GPa}$ and then to a cubic- $\mathrm{Fm}-3 m$ phase at $10 \mathrm{GPa}[79,152]$. The cubic phase of $\mathrm{NaBH}_{4}$ transforms into a tetragonal- $\mathrm{P}^{-} 42_{1} \mathrm{c}$ structure above $6 \mathrm{GPa}$ and then to an orthorhombic- Pnma phase above $8 \mathrm{GPa}$ [84]. $\mathrm{KBH}_{4}$ exhibits structural transitions from a cubic- $\mathrm{F} m-3 m$ to a tetragonal- $P^{-} 42_{1} c$ phase at 3.8 GPa and then to an orthorhombic- Pnma phase at $6.8 \mathrm{GPa}$ [87].

The study of structural stability of $\mathrm{RbBH}_{4}$ at room temperature shows a transition from the ambient cubic- $\mathrm{Fm}^{-} 3 \mathrm{~m}$ phase to an orthorhombic- Pnma phase around $2.5 \mathrm{GPa}$, and then to a monoclinic- P21/c phase above $8 \mathrm{GPa}$ [90]. The reduction in the cubic to tetragonal transition pressure and the absence of a tetragonal high-pressure phase in $\mathrm{RbBH}_{4}$ suggest that the transition pressure is influenced by the size of the alkali atom during the structural rearrangement of $\mathrm{BH}_{4}{ }^{-}$anions and alkali cations. A comparison of the transition pressure among first group metal borohydrides shows that the increase in the size of the alkali cation leads to a decrease in the transition pressure. A lower bulk modulus and a large volume collapse during the first transition imply that both the $\mathrm{Rb}-\mathrm{H}$ bonds and the $\mathrm{BH}_{4}{ }^{-}$units are more compressible and rearranged than that of $\mathrm{NaBH}_{4}$. This 
may favor a direct transition from the cubic phase to the orthorhombic phase without an intermediate structure. Raman spectra as a function of temperature measured for these cubic borohydrides by Hageman et al. [254], also suggest a similar scenario where the energy barrier of the reorientation of the $\mathrm{BH}_{4}^{-}$anions decreases as a function of cation size. Like $\mathrm{Na}, \mathrm{K}$, and $\mathrm{Rb}$ borohydride counterparts, $\mathrm{CsBH}_{4}$ also crystallizes in $\mathrm{Fm} \overline{3} 3 \mathrm{~m}$ structure at room temperature with a disordered $\mathrm{BH}_{4}{ }^{-}$complex [89]. Low temperature order-disorder phase transitions are observed in the alkali borohydrides, which ranges from $-83{ }^{\circ} \mathrm{C}$ for $\mathrm{NaBH}_{4}$ to $-246{ }^{\circ} \mathrm{C}$ for $\mathrm{CsBH}_{4}$ [88]. Compared to other alkali metal borohydrides, $\mathrm{CsBH}_{4}$ has a higher symmetry structure [91]. High temperature Raman spectroscopic studies of alkali borohydrides show $\mathrm{BH}_{4}^{-}$ions are easily reoriented in $\mathrm{CsBH}_{4}[254]$.

Among alkali- earth metal borohydrides, $\mathrm{Be}\left(\mathrm{BH}_{4}\right)_{2}$ is found to be of tetragonalI4 ${ }_{1} \mathrm{~cd}$ structure with a covalent bonding. The estimated decomposition temperature of $\mathrm{Be}\left(\mathrm{BH}_{4}\right)_{2}$ is $-111{ }^{\circ} \mathrm{C}$, even though the compound is found to be stable at room temperature $[36,92]$. The crystal structure and the electronic density of states suggest formation of $\left[-\mathrm{BH}_{4}-\mathrm{Be}^{-}\right] n$ helical polymers. In general $\mathrm{BH}_{4}{ }^{-}$units in borohydrides have a tetragonal co-ordination, whereas in $\mathrm{Be}\left(\mathrm{BH}_{4}\right)_{2}$ it has a trigonal co-ordination [99]. Dehydrogenation of $\mathrm{Be}\left(\mathrm{BH}_{4}\right)_{2}$ to elements is more favorable than that to $\mathrm{BeH}_{2}$ unlike other complex hydrides. $\mathrm{Ca}\left(\mathrm{BH}_{4}\right)_{2}$ has an orthorhombic- F2dd and $\mathrm{Mg}\left(\mathrm{BH}_{4}\right)_{2}$ has a complex hexagonal structure with space group $\mathrm{P} 6_{1} 22$ and both of them exhibits polymorphism $[94,97,160]$. Therefore it is interesting to study structural behavior of metal borohydrides under various conditions of pressures and temperatures. Above 2.5 GPa magnesium borohydride transform to a high pressure phase with $\mathrm{P} 6_{3}$ structure which 
is metastable at ambient conditions. Raman spectroscopic studies show another structural transition of $\mathrm{Mg}\left(\mathrm{BH}_{4}\right)_{2}$ above $14 \mathrm{GPa}$ to an unidentified phase. At $180{ }^{\circ} \mathrm{C}$ an irreversible transition of $\mathrm{Mg}\left(\mathrm{BH}_{4}\right)_{2}$ which occurs from $\mathrm{P}_{1} 22$ to an orthorhombic- Fddd phase. The Fddd phase of $\mathrm{Ca}\left(\mathrm{BH}_{4}\right)_{2}$ transforms to a $\mathrm{F} 2 \mathrm{dd}$ structure at $-182{ }^{\circ} \mathrm{C}$. At high temperatures, three tetragonal phases of $\mathrm{Ca}\left(\mathrm{BH}_{4}\right)_{2}$ are found which are $\mathrm{P}^{-} 4\left(\sim 32{ }^{\circ} \mathrm{C}\right), \mathrm{P}^{-} 4 / 2 \mathrm{~m}\left(207{ }^{\circ} \mathrm{C}\right)$ and $\mathrm{I}^{-} 42 \mathrm{~d}\left(222{ }^{\circ} \mathrm{C}\right)$. The $\mathrm{P}^{-} 42 / \mathrm{m}$ phase is stable at ambient conditions and formed as a result of synthesizing and processing conditions. Mechano- chemical synthesize of $\mathrm{Ca}\left(\mathrm{BH}_{4}\right)_{2}$ show formation of a small amount of Pbca with the Fddd phase [98].

Except $\mathrm{LiAlH}_{4}$ (monoclinic) and $\mathrm{NaAlH}_{4}$ (tetragonal), alkali alanates are of orthorhombic symmetry. Around $3 \mathrm{GPa}$ the ambient monoclinic- $P 2_{1} / c$ phase of $\mathrm{LiAlH}_{4}$ transform to a high pressure phase with a distorted $\mathrm{AlH}_{4}{ }^{-}$tetrahedron $[69,70]$. $\mathrm{NaAlH}_{4}$ undergoes a phase transformation from a tetragonal- $I 4_{1} / a$ to an orthorhombic- $C m c 2_{1}$ structure at $6.43 \mathrm{GPa}$ with $45 \%$ volume contraction [71]. Experimental study of Kumar et al. [73], reports a tetragonal to monoclinic of $\mathrm{NaAlH}_{4}$ phase transition above $14 \mathrm{GPa}$. First principle density functional theory studies show that $\mathrm{RbAlH}_{4}$ and $\mathrm{CsAlH}_{4}$ exist in orthorhombic- Pnma structure at room temperature [26]. Computation of crystallographic data suggest that at ambient conditions, $\mathrm{Mg}\left(\mathrm{AlH}_{4}\right)_{2}$ has a trigonal- $\mathrm{P}^{-} 3 \mathrm{~m}_{1}$ structure which transform to a monoclinic- $\mathrm{C} 2 / \mathrm{m}$ structure around $0.68 \mathrm{GPa}$. Above $10.28 \mathrm{GPa}$, transition to an orthorhombic- Pbca structure is expected [77]. The decomposition of $\operatorname{Mg}\left(\mathrm{AlH}_{4}\right)_{2}$ occurs in three steps, first in the range $110-200^{\circ} \mathrm{C}$, and second and third around 240- 380 and $400{ }^{\circ} \mathrm{C}$, respectively [31]. Density functional theory calculations predict that $\mathrm{Ca}\left(\mathrm{AlH}_{4}\right)_{2}$ is a insulator with orthorhombic- Pbca structure [33]. 
The bulk modulus of several borohydrides and alanates has been determined by high pressure studies. Among that, $\mathrm{LiBH}_{4}$ has bulk modulus values in the range $14.4-26 \mathrm{GPa}$ for three different phases [79]. For $\mathrm{KBH}_{4}$, the $\mathrm{K}_{0}$ is found to be $16.8 \mathrm{GPa}$ [87], whereas for $\mathrm{RbBH}_{4}$ it is $14.5 \mathrm{GPa}$ [90]. The orthorhombic phase of $\mathrm{Ca}\left(\mathrm{BH}_{4}\right)_{2}$ and the high pressure phase of $\mathrm{Mg}\left(\mathrm{BH}_{4}\right)_{2}$ have bulk moduli of 22.9 and $10.16 \mathrm{GPa}$, respectively [ 94 , 97]. Alanates of alkali and alkali- earth metals also has experimental and computed values of bulk modulus in the range 8- $35 \mathrm{GPa}$ [69-71, 73, 75-78]. Among alkali-earth compounds, studies of $\mathrm{Sr}$ and $\mathrm{Ba}$ based complex hydrides are lacking. It is interesting to note that all these borohydrides and alanates have bulk modulus in the same range and they are highly compressible. Figure 12.15 shows the change in molar volume under applied pressure for various complex hydrides. Results show that the borohydrides and alanates are highly compressible solids and hence they may be destabilized under pressure with additives/catalysts to enhance the hydrogenation kinetics. The reordering of Al-H and B-H bonds in the complex hydrides is responsible for the observed phase transitions in them. 


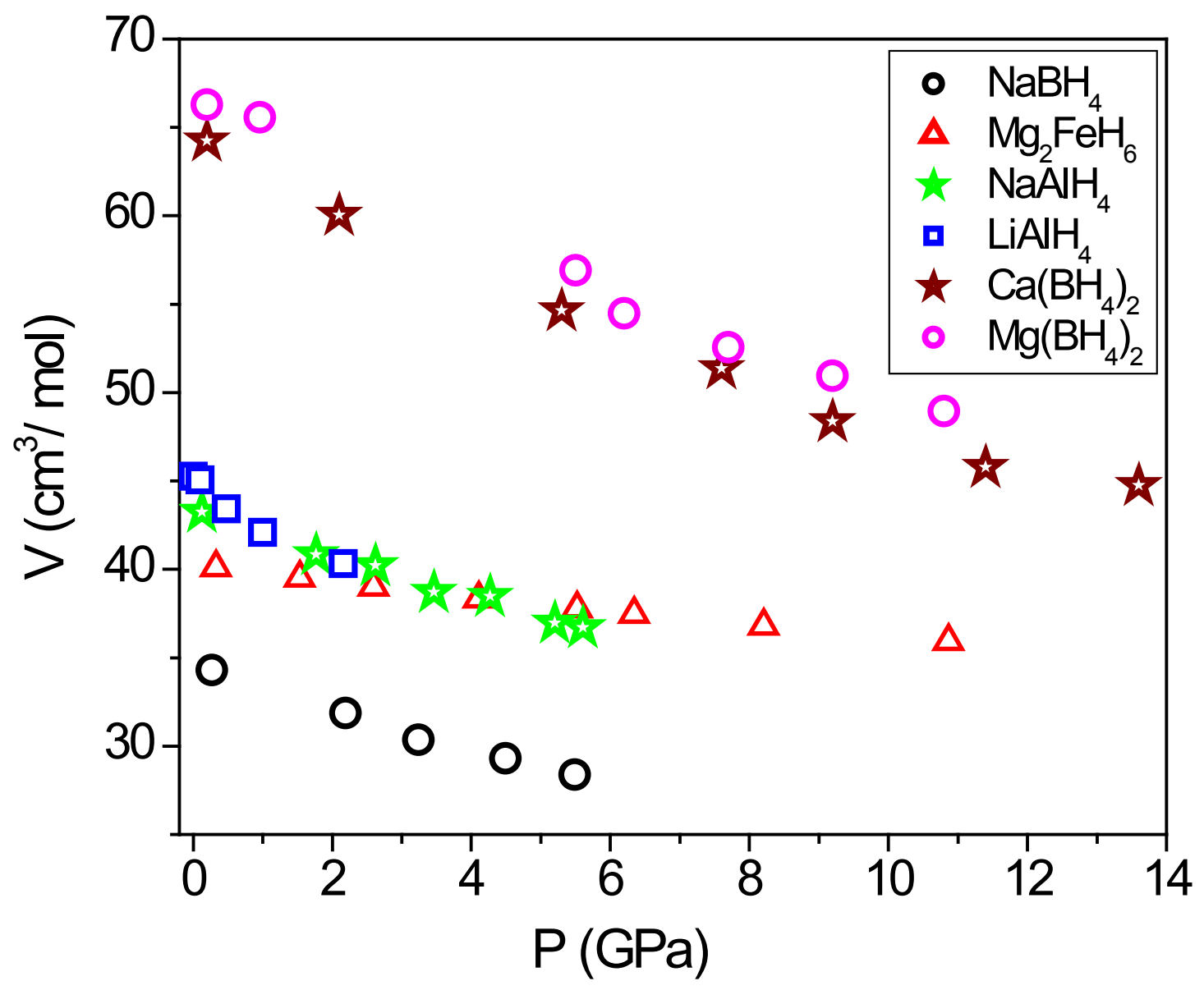

Figure 12. 15: A comparison of the change of molar volume with pressure for the complex hydrides investigated in this study. 


\section{CHAPTER 13. SUMMARY}

Important results derived from this dissertation are concluded below. These include inferences from the high pressure and temperature investigations of $\mathrm{Mg}_{2} \mathrm{FeH}_{6}$, $\mathrm{Mg}\left(\mathrm{BH}_{4}\right)_{2}, \mathrm{Ca}\left(\mathrm{BH}_{4}\right)_{2}, \mathrm{NaBH}_{4}, \mathrm{NaAlH}_{4}, \mathrm{LiAlH}_{4}, \mathrm{LiNH}_{2} \mathrm{BH}_{3}$ and mixture of $\mathrm{MgH}_{2}$ with $\mathrm{AlH}_{3}$ or Si. Interesting features derived from the study of inter- relationships of various properties of hydrides are also summarized.

\subsection{Conclusions}

High pressure phase transitions and physical properties of the selected complex hydrides were investigated with X-ray diffraction and Raman spectroscopy studies. The observed phase transitions and structural data are summarized in the Table 13.1. High pressure phase transitions of alkali metal complex hydrides are found to be first order and fully reversible whereas that of the alkali-earth metal complex hydrides are irreversible. A P-T phase diagram of $\mathrm{NaBH}_{4}$ was also constructed which confirms all the transitions observed for this compound were first order and exothermic. $\mathrm{Mg}_{2} \mathrm{FeH}_{6}$ did not show any phase transition up to the highest pressure reached which indicate high stability of Fe-H bonds. Transition pressure is influenced by the size of alkali atom and the rearrangement of anions and cations. A comparison of the bulk modulus values indicates that complex hydrides are more compressible than their metals and metal hydrides. The reordering of the highly compressible $\mathrm{Al}-\mathrm{H}$ and $\mathrm{B}-\mathrm{H}$ bonds in alanates and borohydrides, respectively are responsible for the observed phase transitions in these compounds. With high 
capacity and favorable thermodynamics, $\mathrm{Mg}\left(\mathrm{BH}_{4}\right)_{2}$ and $\mathrm{Ca}\left(\mathrm{BH}_{4}\right)_{2}$ are suitable for reversible hydrogen storage.

Large thermal expansion observed for the hydrides can be due to the hydrogen induced optical vibration. Bulk modulus values of ball milled sample were found to be higher than that of the unmilled sample. This can be attributed to the particle size reduction and related stress effect due to milling. It is also noted that the alanates and borohydrides have bulk modulus in the same range and they are highly compressible. The high compressibility of the complex metal hydrides suggests that tuning of electron density is possible in these compounds under pressure. Therefore, alanates and borohydrides can be destabilized with additives/catalysts under pressure to enhance the hydrogenation kinetics. On a comparison with the literature data, the decomposition temperature and bulk modulus of alkali metal complex hydrides are found to be increasing with the size of the metal atom due to stronger covalent and ionic bonding in them. The transition pressure is found to be independent of size of metal atoms in complex hydrides unlike the metal hydrides. Bulk modulus of alkali and alkali earth hydrides are higher than that of corresponding metals, suggesting a hydrogen induced hardening of these materials. B-H bond energy is larger than the Al-H bond energy and hence the bulk modulus of borohydrides is higher than that of their alanate counterparts. An unidentified new phase was formed in the high pressure- temperature study of the mixture of $\mathrm{AlH}_{3}$ and $\mathrm{MgH}_{2}$. Investigation of $\mathrm{LiNH}_{2} \mathrm{BH}_{3}$ with high pressure Raman spectroscopy show phase transitions approximately at 5, 10 and $15 \mathrm{GPa} \cdot \mathrm{MgH}_{2}$ was destabilized by Si and formed $\mathrm{Mg}_{2} \mathrm{Si}$ at $0.5 \mathrm{GPa}$ and $600{ }^{\circ} \mathrm{C}$. 
Table 13.1. Summary of the results obtained from the high pressure and/or temperature investigations of the complex hydrides. Data marked * are of ball milled sample.

\begin{tabular}{|c|c|c|c|c|c|c|}
\hline Hydride & $\begin{array}{l}\mathbf{P} \\
(\mathbf{G P a})\end{array}$ & $\begin{array}{l}\text { Structure/ Space } \\
\text { group }\end{array}$ & $\begin{array}{l}K_{0} \\
(\mathbf{G P a})\end{array}$ & $\mathbf{K}_{\mathbf{0}}^{\prime}$ & $\begin{array}{l}\mathbf{V}_{\mathbf{0}} / \mathbf{f} \cdot \mathbf{u} \\
\left(\AA^{\mathbf{3}}\right)\end{array}$ & $\left(10^{-5} K^{-1}\right)$ \\
\hline $\mathrm{LiAlH}_{4}$ & $\begin{array}{l}0-2.16 \\
>3\end{array}$ & $\begin{array}{l}\text { Monoclinic/ P2/c } \\
\text { Tetragonal/ I4 } / \mathrm{a}\end{array}$ & 13.87 & 4 & 75.15 & \\
\hline $\mathrm{NaAlH}_{4}$ & $\begin{array}{l}0-6 \\
>6.3\end{array}$ & $\begin{array}{l}\text { Tetragonal/ } \mathrm{I} 4_{1} / \mathrm{a} \\
\text { Orthorhombic/Cmc } 2_{1}\end{array}$ & $\begin{array}{l}25.3 \\
52.2 *\end{array}$ & 4 & 72.08 & \\
\hline $\mathrm{NaBH}_{4}$ & $\begin{array}{l}0-6.3 \\
6.3-8.9 \\
8.9-14 \\
>14\end{array}$ & $\begin{array}{l}\text { Cubic/ Fm } 3 \mathrm{~m} \\
\text { Tetragonal/ } \mathrm{P}^{-} 42_{1} \mathrm{c} \\
\text { Orthorhombic/ Pnma } \\
\text { Monoclinic/ } \mathrm{P} 2_{1} / \mathrm{c}\end{array}$ & 18.76 & 3.5 & 57.95 & 12.06 \\
\hline $\mathrm{Mg}\left(\mathrm{BH}_{4}\right)_{2}$ & $\begin{array}{l}0-2.5 \\
2.5-14 \\
>14\end{array}$ & $\begin{array}{l}\text { Hexagonal/ } \mathrm{P}_{1} 22 \\
\text { Hexagonal/ } \mathrm{P} 6_{3} \\
\text { Unidentified }\end{array}$ & 10.16 & 4 & 94.53 & \\
\hline $\mathrm{Ca}\left(\mathrm{BH}_{4}\right)_{2}$ & $\begin{array}{l}>0 \\
0-10.2\end{array}$ & $\begin{array}{l}\text { Orthorhombic/ F2dd } \\
\text { Tetragonal/ } 4_{2} / \mathrm{m}\end{array}$ & 22.95 & 2.6 & 108.26 & \\
\hline $\mathrm{Mg}_{2} \mathrm{FeH}_{6}$ & $0-36$ & Cubic/ $\mathrm{Fm}^{-3} \mathrm{~m}$ & $\begin{array}{l}75.4 \\
139^{*}\end{array}$ & 4 & 66.95 & 5.85 \\
\hline
\end{tabular}

Entropy, molar volume, specific heat and hydrogen content of both binary and ternary metal hydrides were analyzed with principal component analysis method. The comparison of properties between metals and their corresponding hydrides gives insight into a combined effect of charge transfer between metal and $\mathrm{H}$-atom, and structural transformation. The specific heat of binary hydrides is higher than that of the corresponding metals, whereas the molar volume and entropy of certain hydrides were less than that of corresponding metals which suggests an interrelationship between crystal chemistry and hydrogen induced properties. The average value of molar volume of $\mathrm{H}$ atom in the boron hydrides was determined to be $\sim 6 \mathrm{~cm}^{3} / \mathrm{mol}$. The thermodynamic 
properties of various metal hydrides were correlated and found many interesting features. The entropy- molar volume relationship of hydrides is found to be different from that of the host metals. Entropy of a hydride can be related to the molar volume of the metal by the equation: $\mathrm{S}_{\mathrm{MH}}=-3.074+2.153\left(\mathrm{~V}_{\mathrm{M}}+\mathrm{V}_{\mathrm{H}}+\Delta\right)$. Larger the difference between electronegativities of metal and hydrogen, greater will be the charge transfer effects $(\Delta)$ and hence the change of entropy of the hydride.

\subsection{Recommendations for Future Research}

o The hydrides investigated are promising hydrogen storage materials. Further studies are required to understand the mechanism to overcome kinetic barriers of dehydrogenation of the complex hydrides. The additives should be selected such that it weakens the B-H and $\mathrm{Al}-\mathrm{H}$ bonds in the borohydrides and alanates, respectively. Improved hydrogenation properties are expected for the complex hydrides owing to their high compressibility which should be investigated under pressure. The decomposition temperature of the high pressure phases of the hydrides which are metastable at ambient conditions has to be determined to confirm whether they are usable.

o Some of the complex hydrides like $\mathrm{LiAlH}_{4}$ exhibit high ionic conductivity and hence potential for their application as a solid electrolyte should be investigated.

- Further studies of $\operatorname{Mg}\left(\mathrm{AlH}_{4}\right)_{2}$ has to be carried out to determine the structure of the high pressure phase which was observed above $0.68 \mathrm{GPa}$. The influence of synthesis and processing conditions on the crystal structure of $\operatorname{Mg}\left(\mathrm{AlH}_{4}\right)_{2}$ is interesting as the 
borohydride counterpart show irreversible phase transitions under pressure and temperature.

- The new phase formed in the high P-T study of the mixture of $\mathrm{AlH}_{3}$ and $\mathrm{MgH}_{2}$ has to be identified using high quality data such as neutron diffraction.

- The XRD patterns of the mixture of $\mathrm{Si}$ and $\mathrm{LiAlH}_{4}$ after heating to $500{ }^{\circ} \mathrm{C}$ show formation of a new phase above $11.2 \mathrm{GPa}$. Hence it will be interesting to study the structure and thermodynamic stability of this mixture.

- A mixture of $\mathrm{Si}$ and $\mathrm{H}_{2} \mathrm{O}$ was studied under high temperature using a moissanite anvil cell up to $600{ }^{\circ} \mathrm{C}$. The sample at $1.5 \mathrm{GPa}$ was heated with a gas blower. A reaction between $\mathrm{Si}$ and $\mathrm{H}_{2} \mathrm{O}$ might have occurred as can be concluded from the appearance of a new peak in the Raman spectroscopy experiment. The unidentified phase should be further investigated with large amount of sample to get good quality X-ray diffraction patterns for further analysis.

- $\mathrm{LiNH}_{2} \mathrm{BH}_{3}$ was investigated with high pressure Raman spectroscopy and found phase transitions approximately at 5, 10 and $15 \mathrm{GPa}$. Phase identification of $\mathrm{LiNH}_{2} \mathrm{BH}_{3}$ has to be carried out with high quality X-ray diffraction or neutron diffraction.

○ $\mathrm{MgH}_{2}$ was destabilized by $\mathrm{Si}$ and formed $\mathrm{Mg}_{2} \mathrm{Si}$ at $0.5 \mathrm{GPa}$ and $600{ }^{\circ} \mathrm{C}$. Hydrogenation of $\mathrm{Mg}_{2} \mathrm{Si}$ under high $\mathrm{H}_{2}$ pressure and temperature will need to identify potential of this mixture.

- The high pressure structural data of alkali-earth alanates and borohydrides are scarce owing to their high reactivity and difficulty to synthesize. Alanates and borohydrides of $\mathrm{Sr}$ and $\mathrm{Ba}$ have to be investigated under pressure and temperature to draw a general conclusion from their structural properties. 


\section{LIST OF REFERENCES}

[1] F.Waelbroeck, P.Wienhold, J.Winter, Journal of Nuclear Materials 2011, 111 $112,185$.

[2] J.Kleperis, G.Wojcik, A.Czerwinski, J.Skowronski, M.Kopczyk, B.M.Beltowska, Journal of Solid State Electrochemistry 2001, 5, 229.

[3] V.E.Antonov, Journal of Alloys and Compounds 2002, 330-332, 110.

[4] B.Baranowski, S.Filipek, Journal of the Less Common Metals 1982, 88, 290.

[5] B.Baranowski, Journal of the Less Common Metals 1984, 101, 115.

[6] T.Palasyuk, M.Tkacz, P.Vajda, Solid State Communications 2005, 135, 226.

[7] M.Tkacz, The Journal of Chemical Thermodynamics 2001, 33, 891.

[8] M.Tkacz, R.Burtovyy, Solid State Communications 2004, 132, 37.

[9] W.Grochala, P.P.Edwards, Chemical Reviews 2004, 104, 1283.

[10] H.Smithson, C.A.Marianetti, D.Morgan, A.Van der Ven, A.Predith, G.Ceder, Physical Review B 2002, 66, 144107.

[11] C.Y.Xiao, J.L.Yang, K.M.Deng, Z.H.Bian, K.L.Wang, Journal of Physics: Condensed Matter 1994, 6, 8539.

[12] T.Noritake, M.Aoki, S.Towata, Y.Seno, Y.Hirose, E.Nishibori, M.Takata, M.Sakata, Applied Physics Letters 2002, 81, 2008.

[13] R.Yu, P.K.Lam, Physical Review B 1988, 37, 8730.

[14] P.Vajeeston, P.Ravindran, A.Kjekshus, H.Fjellvag, Applied Physics Letters 2004, 84,34 .

[15] "New World Encyclopedia", 2008.

[16] L.George, R.Hrubiak, K.Rajan, S.K.Saxena, Journal of Alloys and Compounds 2009, 478, 731 .

[17] R.Gupta, F.Agresti, S.Lo Russo, A.Maddalena, P.Palade, G.Principi, Journal of Alloys and Compounds 2008, 450, 310.

[18] J.Huot, I.Swainson, R.Schulz, Annales de Chimie-Science des Materiaux 2006, $31,135$. 
[19] S.A.Jin, Y.S.Lee, J.H.Shim, Y.W.Cho, Journal of Physical Chemistry C 2008, 112,9520 .

[20] X.Kang, P.Wang, H.Cheng, Rare Metals 2006, 25, 266.

[21] O.M.Lovvik, Journal of Alloys and Compounds 2004, 373, 28.

[22] M.Yoshino, K.Komiya, Y.Takahashi, Y.Shinzato, H.Yukawa, M.Morinaga, Journal of Alloys and Compounds 2005, 404-406, 185.

[23] Z.F.Hou, Journal of Power Sources 2006, 159, 111.

[24] V.P.Balema, K.W.Dennis, V.K.Pecharsky, Chemical Communications, 1665.

[25] M.Matsuo, A.Remhof, P.Martelli, R.Caputo, M.Ernst, Y.Miura, T.Sato, H.Oguchi, H.Maekawa, H.Takamura, A.Borgschulte, A.Zuttel, S.i.Orimo, Journal of the American Chemical Society 2009, 131, 16389.

[26] P.Vajeeston, P.Ravindran, R.Vidya, H.Fjellvag, A.Kjekshus, Crystal Growth \& Design 2004, 4, 471.

[27] B.Bogdanovic, M.Schwickardi, Journal of Alloys and Compounds 1997, 253-254, 1.

[28] B.Bogdanovic, R.A.Brand, A.Marjanovic, M.Schwickardi, J.Tolle, Journal of Alloys and Compounds 2000, 302, 36.

[29] F.Schuth, B.Bogdanovic, M.Felderhoff, Chemical Communications 2004, 2249.

[30] H.Morioka, K.Kakizaki, S.C.Chung, A.Yamada, Journal of Alloys and Compounds 2003, 353, 310.

[31] M.Fichtner, O.Fuhr, O.Kircher, Journal of Alloys and Compounds 2003, 356-357, 418.

[32] A.Fossdal, H.W.Brinks, M.Fichtner, B.C.Hauback, Journal of Alloys and Compounds 2005, 387, 47.

[33] O.M.Lovvik, Physical Review B 2005, 71, 1144111.

[34] L.Mosegaard, B.Moller, J.E.Jorgensen, U.Bosenberg, M.Dornheim, J.C.Hanson, Y.Cerenius, G.Walker, H.J.Jakobsen, F.Besenbacher, T.R.Jensen, Journal of Alloys and Compounds 2007, 446-447, 301.

[35] A.G.Ostroff, R.T.Sanderson, Journal of Inorganic and Nuclear Chemistry 1957, $4,230$. 
[36] M.J.van Setten, G.A.de Wijs, G.Brocks, Physical Review B 2008, 77, 165115.

[37] J.Voss, J.S.Hummelshoj, Z.Lodziana, T.Vegge, Journal of Physics: Condensed Matter 2009, 21, 012203.

[38] T.Matsunaga, F.Buchter, P.Mauron, M.Bielman, Y.Nakamori, S.Orimo, N.Ohba, K.Miwa, S.Towata, A.Znttel, Journal of Alloys and Compounds 2008, 459, 583.

[39] J.H.Kim, S.A.Jin, J.H.Shim, Y.W.Cho, Journal of Alloys and Compounds 2008, 461, L20-L22.

[40] L.Liu, W.A.Bassett, Elements, oxides, Silicates: High pressure phases with implications for the earth's interior, Oxford University Press, USA, 1986.

[41] R.Ahuja, O.Eriksson, B.Johansson, Physica B 1999, 265, 87.

[42] F.D.Murnaghan, Proceedings of the National Academy of Sciences of the United States of America 1944, 30, 244.

[43] F.Birch, Journal of Geophysical research 1978, 83, 1257.

[44] F.Birch, Physical Review 1947, 71, 809.

[45] Y.Filinchuk, D.Chernyshov, R.Cerny, The Journal of Physical Chemistry C 2008, $112,10579$.

[46] R.Ahuja, O.Eriksson, J.M.Wills, B.Johansson, Journal of Physics: Condensed Matter 1998, 10, L153-L158.

[47] S.Filipek, Journal of Advanced Science 2007, 19, 1.

[48] S.J.Duclos, Y.K.Vohra, A.L.Ruoff, S.Filipek, B.Baranowski, Physical Review B 1987, 36, 7664 .

[49] I.O.Bashkin, T.N.Dymova, E.G.Ponyatovskii, Physica Status Solidi B 2006, 100, 87.

[50] J.J.Vajo, F.Mertens, C.C.Ahn, C.Robert, B.Fultz, The Journal of Physical Chemistry B 2004, 108, 13977.

[51] D.R.Stephens, E.M.Lilley, J. Appl. Phys. 1968, 39, 177.

[52] B.Olinger, P.M.Halleck, Applied Physics Letters 1974, 24, 536.

[53] G.Li, D.Wang, Q.Jin, D.Ding, Physics Letters A 1990, 143, 473.

[54] J.Sangster, A.Pelton, Journal of Phase Equilibria 1997, 18, 387. 
[55] J.Sangster, A.Pelton, Journal of Phase Equilibria 1994, 15, 87.

[56] J.Songster, A.Pelton, Journal of Phase Equilibria 1994, 15, 84.

[57] K.Ghandehari, H.Luo, A.L.Ruoff, S.S.Trail, F.J.Disalvo, Physical Review Letters 1995, 74, 2264.

[58] U.Hantsch, B.Winkler, V.Milman, Chemical Physics Letters 2003, 378, 343.

[59] M.Ahart, J.L.Yarger, K.M.Lantzky, S.Nakano, H.K.Mao, R.J.Hemley, Journal of Chemical Physics 2006, 124, 014502.

[60] P.Vajeeston, P.Ravindran, B.C.Hauback, H.Fjellvag, A.Kjekshus, S.Furuseth, M.Hanfland, Physical Review B 2006, 73, 224102.

[61] J.Hector, J.F.Herbst, W.Wolf, P.Saxe, G.Kresse, Physical Review B 2007, 76, 014121.

[62] J.S.Tse, D.D.Klug, S.Desgreniers, J.S.Smith, R.Flacau, Z.Liu, J.Hu, N.Chen, D.T.Jiang, Physical Review B 2007, 75, 134108.

[63] B.Li, Y.W.Li, K.F.Yang, Q.L.Cui, Y.M.Ma, G.T.Zou, Journal of PhysicsCondensed Matter 2007, 19, 226205.

[64] Y.W.Li, B.Li, T.Cui, Y.Li, L.J.Zhang, Y.M.Ma, G.T.Zou, Journal of PhysicsCondensed Matter 2008, 20, 045211.

[65] J.S.Smith, S.Desgreniers, D.D.Klug, J.S.Tse, Solid State Communications 2009, 149,830 .

[66] K.Kinoshita, M.Nishimura, Y.Akahama, H.Kawamura, Solid State Communications 2007, 141, 69.

[67] J.S.Smith, S.Desgreniers, J.S.Tse, D.D.Klug, Journal of Applied Physics 2007, 102,043520 .

[68] W.Luo, R.Ahuja, Journal of Alloys and Compounds 2007, 446, 405.

[69] R.S.Chellappa, D.Chandra, S.A.Gramsch, R.J.Hemley, J.F.Lin, Y.Song, Journal of Physical Chemistry B 2006, 110, 11088.

[70] A.V.Talyzin, B.Sundqvist, Physical Review B 2004, 70, 180101.

[71] P.Vajeeston, P.Ravindran, R.Vidya, H.Fjellvag, A.Kjekshus, Physical Review B 2003, 68, 212101.

[72] B.Bulychev, Russian Journal of Inorganic Chemistry 1977, 22, 2461. 
[73] R.S.Kumar, E.Kim, O.Tschauner, A.L.Cornelius, M.P.Sulic, C.M.Jensen, Physical Review B 2007, 75, 174110.

[74] P.Vajeeston, P.Ravindran, R.Vidya, H.Fjellvag, A.Kjekshus, Applied Physics Letters 2003, 82, 2257.

[75] P.Vajeeston, P.Ravindran, A.Kjekshus, H.Fjellvag, Journal of Alloys and Compounds 2004, 363, L7-L11.

[76] P.Ravindran, P.Vajeeston, R.Vidya, H.Fjellvag, A.Kjekshus, Journal of Power Sources 2006, 159, 88.

[77] C.H.Hu, D.M.Chen, Y.M.Wang, D.S.Xu, K.Yang, Journal of Physics-Condensed Matter 2007, 19, 176205.

[78] P.Vajeeston, P.Ravindran, A.Kjekshus, H.Fjellvag, Applied Physics Letters 2006, 89, 071906.

[79] Y.Filinchuk, D.Chernyshov, A.Nevidomskyy, V.Dmitriev, Angewandte ChemieInternational Edition 2008, 47, 529.

[80] A.V.Talyzin, O.Andersson, B.Sundqvist, A.Kurnosov, L.Dubrovinsky, Journal of Solid State Chemistry 2007, 180, 510.

[81] T.J.Frankcombe, G.J.Kroes, Physical Review B 2006, 73, 174302.

[82] M.R.Hartman, J.J.Rush, T.J.Udovic, J.Bowman, S.J.Hwang, Journal of Solid State Chemistry 2007, 180, 1298.

[83] J.P.Soulie, G.Renaudin, R.Cerny, K.Yvon, Journal of Alloys and Compounds 2002, 346, 200.

[84] E.Kim, R.Kumar, P.F.Weck, A.L.Cornelius, M.Nicol, S.C.Vogel, J.Z.Zhang, M.Hartl, A.C.Stowe, L.Daemen, Y.S.Zhao, Journal of Physical Chemistry B 2007, 111, 13873.

[85] R.S.Kumar, A.L.Cornelius, Applied Physics Letters 2005, 87, 261916.

[86] L.George, V.Drozd, H.Couvy, J.Chen, S.K.Saxena, The Journal of Chemical Physics 2009, 131, 074505.

[87] R.S.Kumar, E.Kim, A.L.Cornelius, Journal of Physical Chemistry C 2008, 112, 8452 .

[88] C.C.Stephenson, D.W.Rice, W.H.Stockmayer, The Journal of Chemical Physics 1955, 23, 1960. 
[89] G.Renaudin, S.Gomes, H.Hagemann, L.Keller, K.Yvon, Journal of Alloys and Compounds 2004, 375, 98.

[90] R.S.Kumar, A.L.Cornelius, Journal of Alloys and Compounds 2009, 476, 5.

[91] P.Vajeeston, P.Ravindran, A.Kjekshus, H.Fjellvag, Journal of Alloys and Compounds 2005, 387, 97.

[92] D.S.Marynick, W.N.Lipscomb, Inorganic Chemistry 2002, 11, 820.

[93] Y.Filinchuk, R.Cerny, H.Hagemann, Chemistry of Materials 2009, 21, 925.

[94] L.George, V.Drozd, S.K.Saxena, E.G.Bardaji, M.Fichtner, Journal of Physical Chemistry C 2009, 113, 486.

[95] J.H.Her, P.W.Stephens, Y.Gao, G.L.Soloveichik, J.Rijssenbeek, M.Andrus, J.C.Zhao, Acta Crystallographica Section B 2007, 63, 561.

[96] Y.Filinchuk, E.Ronnebro, D.Chandra, Acta Materialia 2009, 57, 732.

[97] L.George, V.Drozd, S.K.Saxena, E.G.Bardaji, M.Fichtner, The Journal of Physical Chemistry C 2009, 113, 15087.

[98] F.Buchter, Z.Lodziana, A.Rernhof, O.Friedrichs, A.Borgschulte, P.Mauron, A.Zuttel, D.Sheptyakov, G.Barkhordarian, R.Bormann, K.Chlopek, M.Fichtner, M.Sorby, M.Riktor, B.Hauback, S.Orimo, Journal of Physical Chemistry B 2008, 112,8042 .

[99] Y.Filinchuk, D.Chernyshov, V.Dmitriev, Zeitschrift fur Kristallographie 2008, 223,649 .

[100] T.Hayashi, K.Tobita, S.Nishio, K.Ikeda, Y.Nakamori, S.Orimo, Fusion Engineering and Design 2006, 81, 1285.

[101] "http://hydpark.ca.sandia.gov/DBFrame.html", 2010.

[102] "http://www1.eere.energy.gov/hydrogenandfuelcells/storage.html”, 2010.

[103] W.A.Bassett, High Pressure Research: An International Journal 2009, 29, 163.

[104] H.K.Mao, J.Xu, P.M.Bell, Journal of Geophysical research 1986, 91, 4673.

[105] I.F.Silvera, A.D.Chijioke, W.J.Nellis, A.Soldatov, J.Tempere, Physica Status Solidi B 2007, 244, 460.

[106] N.M.Balzaretti, E.J.Gonzalez, G.J.Piermarini, T.P.Russell, Review of Scientific Instruments 1999, 70, 4316. 
[107] "http://www.nsls.bnl.gov/beamlines/beamline.asp?blid=X17C", 2009.

[108] A.P.Hammersley, Internal Report ESRF97HA02T; EuropeanSynchrotron Radiation Facility, Grenoble, France 1997.

[109] A.C.Larson, R.B.Von Dreele, Los Alamos National Laboratory Report LAUR 2004, 86 .

[110] "http://www.ccp14.ac.uk/solution/gsas/", 2010.

[111] "http://www.ncnr.nist.gov/xtal/software/gsas.html", 2010.

[112] "http://sbmp95.ess.sunysb.edu/sam85/Plot85/plot85_windows.htm", 2010.

[113] R.J.Angel, High-pressure, high-temperature crystal chemistry 2001, 41, 35.

[114] B.Toby, Journal of Applied Crystallography 2005, 38, 1040.

[115] W.Kraus, G.Nolze, Journal of Applied Crystallography 1996, 29, 301.

[116] "http://www.unipress.waw.pl/fityk/", 2010.

[117] "https://www.spss.com/statistics/", 2010.

[118] B.Bogdanovic, A.Reiser, K.Schlichte, B.Spliethoff, B.Tesche, Journal of Alloys and Compounds 2002, 345, 77.

[119] A.Reiser, B.Bogdanovic, K.Schlichte, International Journal of Hydrogen Energy 2000, 25, 425 .

[120] F.C.Gennari, F.J.Castro, J.J.A.Gamboa, Journal of Alloys and Compounds 2002, 339, 261.

[121] M.Herrich, N.Ismail, A.Handstein, A.Pratt, O.Gutfleisch, Materials Science and Engineering B-Solid State Materials for Advanced Technology 2004, 108, 28.

[122] J.Huot, S.Boily, E.Akiba, R.Schulz, Journal of Alloys and Compounds 1998, 280, 306.

[123] S.L.Li, R.A.Varin, Transactions of Nonferrous Metals Society of China 2004, 14, 649.

[124] S.L.Li, R.A.Varin, O.Morozova, T.Khomenko, Journal of Alloys and Compounds 2004, 384, 231.

[125] T.Ohtsuji, T.Akiyama, J.Yagi, Journal of the Japan Institute of Metals 2000, 64, 656. 
[126] J.A.Puszkiel, P.A.Larochette, F.C.Gennari, Journal of Alloys and Compounds 2008, 463, 134.

[127] J.A.Puszkiel, P.A.Larochette, F.C.Gennari, International Journal of Hydrogen Energy 2008, 33, 3555.

[128] S.S.S.Raman, D.J.Davidson, J.L.Bobet, O.N.Srivastava, Journal of Alloys and Compounds 2002, 333, 282.

[129] P.Selvam, K.Yvon, International Journal of Hydrogen Energy 1991, 16, 615.

[130] C.X.Shang, M.Bououdina, Z.X.Guo, Journal of Alloys and Compounds 2003, 356,626 .

[131] J.J.Didisheim, P.Zolliker, K.Yvon, P.Fischer, J.Schefer, M.Gubelmann, A.F.Williams, Inorganic Chemistry 1984, 23, 1953.

[132] S.V.Halilov, D.J.Singh, M.Gupta, R.Gupta, Physical Review B, 2004,70, 195117.

[133] E.Orgaz, M.Gupta, Journal of Physics-Condensed Matter 1993, 5, 6697.

[134] E.Ronnebro, Y.Fukai, S.Yamamoto, T.Sakai, Journal of Alloys and Compounds 385 (2004) 276-282 2004, 385, 276.

[135] A.C.Larson, R.B.Von Dreele, "General Structure Analysis System (GSAS). Los Alamos National Laboratory Report." 2000.

[136] J.P.Perdew, A.K.Burke, International Journal of Quantum Chemistry 1996, 57, 309.

[137] J.P.Perdew, K.Burke, M.Ernzerhof, Physical Review Letters 1996, 77, 3865.

[138] G.Kresse, J.Furthmnller, Physical Review B 1996, 54, 11169.

[139] P.E.Blochl, Physical Review B 1994, 50, 17953.

[140] V.E.Antonov, M.Baier, B.Dorner, V.K.Fedotov, G.Grosse, A.I.Kolesnikov, E.G.Ponyatovsky, G.Schneider, F.E.Wagner, Journal of Physics: Condensed Matter 2002, 14, 6427.

[141] S.K.Saxena, L.S.Dubrovinsky, P.Haggkvist, Y.Cerenius, G.Shen, H.K.Mao, Science 1995, 269, 1703.

[142] M.M.Barsan, J.Moyer, I.S.Butler, D.F.R.Gilson, Journal of Alloys and Compounds 2006, 424, 73.

[143] L.M.Lawson Daku, H.Hagemann, Physical Review B 2007, 76, 014118. 
[144] C.P.Kempter, R.O.Elliott, J.Gschneidner, The Journal of Chemical Physics 1960, $33,837$.

[145] S.F.Parker, K.P.J.Williams, M.Bortz, K.Yvon, Inorganic Chemistry 1997, 36, 5218.

[146] K.Chlopek, C.Frommen, A.Leon, O.Zabara, M.Fichtner, Journal of Materials Chemistry 2007, 17, 3496.

[147] Y.Nakamori, K.Miwa, A.Ninomiya, H.Li, N.Ohba, S.i.Towata, A.Zuttel, S.i.Orimo, Physical Review B 2006, 74, 045126.

[148] V.Ozolins, E.H.Majzoub, C.Wolverton, Physical Review Letters 2008, 100, 135501.

[149] G.Barkhordarian, T.R.Jensen, S.Doppiu, U.Bosenberg, A.Borgschulte, R.Gremaud, Y.Cerenius, M.Dornheim, T.Klassen, R.Bormann, Journal of Physical Chemistry C 2008, 112, 2743.

[150] J.H.Kim, J.H.Shim, Y.W.Cho, Journal of Power Sources 2008, 181, 140.

[151] E.Ronnebro, E.H.Majzoub, Journal of Physical Chemistry B 2007, 111, 12045.

[152] V.Dmitriev, Y.Filinchuk, D.Chernyshov, A.V.Talyzin, A.Dzwilevski, O.Andersson, B.Sundqvist, Physical Review B 2008, 77, 174112.

[153] R.Cerny, Y.Filinchuk, H.Hagemann, K.Yvon, Angewandte Chemie International Edition 2007, 46, 5765.

[154] H.W.Li, K.Kikuchi, Y.Nakamori, K.Miwa, S.Towata, S.Orimo, Scripta Materialia 2007, 57, 679.

[155] T.Matsunaga, F.Buchter, K.Miwa, S.Towata, S.Orimo, A.Znttel, Renewable Energy 2008, 33, 193.

[156] R.A.Varin, C.Chiu, Z.S.Wronski, Journal of Alloys and Compounds 2008, 462, 201.

[157] P.Zanella, L.Crociani, N.Masciocchi, G.Giunchi, Inorganic Chemistry 2007, 46, 9039.

[158] F.M.Brower, N.E.Matzek, P.F.Reigler, H.W.Rinn, C.B.Roberts, D.L.Schmidt, J.A.Snover, K.Terada, Journal of the American Chemical Society 1976, 98, 2450.

[159] G.Lucazeau, Journal of Raman Spectroscopy 2003, 34, 478. 
[160] K.Miwa, M.Aoki, T.Noritake, N.Ohba, Y.Nakamori, S.Towata, A.Zuttel, S.Orimo, Physical Review B 2006, 74, 155122.

[161] M.Aoki, K.Miwa, T.Noritake, N.Ohba, M.Matsumoto, H.W.Li, Y.Nakamori, S.Towata, S.Orimo, Applied Physics A-Materials Science and Processing 2008, 92, 601 .

[162] Y.S.Lee, Y.Kim, Y.W.Cho, D.Shapiro, C.Wolverton, V.Ozolins, Physical Review B 2009, 79, 104107.

[163] E.H.Majzoub, E.Ronnebro, Journal of Physical Chemistry C 2009, 113, 3352.

[164] M.D.Riktor, M.H.Sorby, K.Chlopek, M.Fichtner, F.Buchter, A.Zuttel, B.C.Hauback, J. Mater. Chem. 2007, 17, 4939.

[165] Z.P.Li, B.H.Liu, K.Arai, K.Asaba, S.Suda, Journal of Power Sources 2004, 126, 28.

[166] E.Shafirovich, V.Diakov, A.Varma, International Journal of Hydrogen Energy 2007, 32, 207.

[167] P.Fischer, A.Zuttel, European Powder Diffraction Epdic 2004, 443-4, 287.

[168] H.L.Johnston, N.C.Hallett, Journal of the American Chemical Society 2002, 75, 1467.

[169] B.Sundqvist, O.Andersson, Physical Review B 2006, 73, 1092102.

[170] G.Lee, J.Y.Lee, J.S.Kim, Solid State Communications 2006, 139, 516.

[171] C.M.Araujo, R.Ahuja, A.V.Talyzin, B.Sundqvist, Physical Review B 2005, 72, 054125 .

[172] Y.Filinchuk, A.V.Talyzin, D.Chernyshov, V.Dmitriev, Physical Review B 2007, 76, 092104.

[173] T.J.Frankcombe, G.J.Kroes, A.Znttel, Chemical Physics Letters 2005, 405, 73.

[174] B.Sundqvist, O.Andersson, A.V.Talyzin, Journal of Physics: Condensed Matter 2007, 19, 425201.

[175] G.Ouyang, C.Q.Sun, W.G.Zhu, The Journal of Physical Chemistry B 2008, 112, 5027.

[176] A.K.Kleppe, A.P.Jephcoat, N.L.Ross, American Mineralogist 2001, 86, 1275.

[177] W.G.Fateley, N.T.McDevitt, F.F.Bentley, Applied Spectroscopy 1971, 25, 155. 
[178] E.Kroumova, M.I.Aroyo, J.M.Perez-Mato, A.Kirov, C.Capillas, S.Ivantchev, H.Wondratschek, Phase Transitions 2003, 76, 155.

[179] D.Errandonea, F.J.Manjon, Progress in Materials Science 2008, 53, 711.

[180] D.L.Anton, Journal of Alloys and Compounds 2003, 356-357, 400.

[181] S.Satyapal, J.Petrovic, C.Read, G.Thomas, G.Ordaz, Catalysis Today 2007, 120, 246.

[182] A.Zaluska, L.Zaluski, J.O.Strom-Olsen, Journal of Alloys and Compounds 2000, $298,125$.

[183] R.A.Zidan, S.Takara, A.G.Hee, C.M.Jensen, Journal of Alloys and Compounds 1999, 285, 119.

[184] R.K.Ahluwalia, International Journal of Hydrogen Energy 2007, 32, 1251.

[185] E.C.Ashby, P.Kobetz, Inorganic Chemistry 1966, 5, 1615.

[186] M.P.Balogh, G.G.Tibbetts, F.E.Pinkerton, G.P.Meisner, C.H.Olk, Journal of Alloys and Compounds 2003, 350, 136.

[187] K.J.Gross, G.J.Thomas, C.M.Jensen, Journal of Alloys and Compounds 2002, 330-332, 683.

[188] A.V.Talyzin, B.Sundqvist, High Pressure Research 2006, 26, 165.

[189] S.Gomes, G.Renaudin, H.Hagemann, K.Yvon, M.P.Sulic, C.M.Jensen, Journal of Alloys and Compounds 2005, 390, 305.

[190] C.M.Jensen, R.Zidan, N.Mariels, A.Hee, C.Hagen, International Journal of Hydrogen Energy 1999, 24, 461.

[191] G.P.Meisner, G.G.Tibbetts, F.E.Pinkerton, C.H.Olk, M.P.Balogh, Journal of Alloys and Compounds 2002, 337, 254.

[192] R.A.Dunlap, Z.H.Cheng, G.R.MacKay, J.W.O'Brien, D.A.Small, Hyperfine Interactions 2000, 130, 109.

[193] J.W.Lauher, D.Dougherty, P.J.Herley, Acta Crystallographica Section B 1979, $35,1454$.

[194] E.Husson, C.Proust, P.Gillet, J.P.Itie, Materials Research Bulletin 2009, 34, 2085.

[195] S.Klotz, G.Hamel, J.S.Loveday, R.J.Nelmes, M.Guthrie, A.K.Soper, Physical Review Letters 2002, 89, 285502. 
[196] D.J.Ross, M.D.Halls, A.G.Nazri, R.F.Aroca, Chemical Physics Letters 2004, 388, 430.

[197] L.C.Ming, S.R.Shieh, A.Jayaraman, S.K.Sharma, Y.H.Kim, Journal of Physics and Chemistry of Solids 1999, 60, 69.

[198] V.Selva, S.R.Kulkarni, S.K.Saxena, H.P.Liermann, S.V.Sinogeikin, Applied Physics Letters 2006, 89, 261901.

[199] Z.Wang, Y.Zhao, D.Schiferl, C.S.Zha, R.T.Downs, T.Sekine, Applied Physics Letters 2003, 83, 3174.

[200] L.Schlapbach, A.Zuttel, Nature (London) 2001, 414, 353.

[201] B.C.Hauback, H.W.Brinks, H.Fjellvag, Journal of Alloys and Compounds 2002, 346, 184.

[202] J.P.Bastide, J.C.Bureau, J.M.Letoffe, P.Claudy, Materials Research Bulletin 1987, 22, 185.

[203] M.P.Pitt, D.Blanchard, B.C.Hauback, H.Fjellvag, W.G.Marshall, Physical Review $B$ 2005, 72, 214113.

[204] S.Wold, K.Esbensen, P.Geladi, Chemometrics and Intelligent Laboratory Systems 1987, 2, 37 .

[205] M.R.Euerby, P.Petersson, Journal of Chromatography A 2003, 994, 13.

[206] K.Heberger, K.Milczewska, A.Voelkel, Colloids and Surfaces A: Physicochemical and Engineering Aspects 2005, 260, 29.

[207] E.R.Malinowski, "Factor Analysis in Chemistry", Wiley NY, 1991.

[208] M.Otto, "Chemometrics: Statistics and Computer Application in Analytical Chemistry", Wiley-VCH, Weinheim, 1999.

[209] K.Polat, S.Gnnes, Applied Mathematics and Computation 2007, 186, 898.

[210] J.Bajorath, Journal of Chemical Information and Computer Sciences 2001, 41, 233.

[211] B.Hess, Physical Review E 2000, 62, 8438.

[212] M.Viana, X.Querol, A.Alastuey, J.I.Gil, M.Menendez, Chemosphere 2006, 65, 2411.

[213] T.J.Clark, G.R.Whittell, I.Manners, Inorganic Chemistry 2007, 46, 7522. 
[214] M.Chandra, Q.Xu, Journal of Power Sources 2007, 168, 135.

[215] M.E.Bluhm, M.G.Bradley, R.Butterick, U.Kusari, L.G.Sneddon, Journal of the American Chemical Society 2006, 128, 7748.

[216] M.C.Denney, V.Pons, T.J.Hebden, D.M.Heinekey, K.I.Goldberg, Journal of the American Chemical Society 2006, 128, 12048.

[217] A.Feaver, S.Sepehri, P.Shamberger, A.Stowe, T.Autrey, G.Cao, The Journal of Physical Chemistry B 2007, 111, 7469 .

[218] J.Baumann, F.Baitalow, G.Wolf, Thermochimica Acta 2005, 430, 9.

[219] X.Kang, Z.Fang, Kong, H.Cheng, X.Yao, G.Lu, P.Wang, Advanced Materials 2008, 20, 2756.

[220] Z.Xiong, C.K.Yong, G.Wu, P.Chen, W.Shaw, A.Karkamkar, T.Autrey, M.O.Jones, S.R.Johnson, P.P.Edwards, W.I.F.David, Nature Materials 2008, 7, 138.

[221] M.Ramzan, F.Silvearv, A.Blomqvist, R.H.Scheicher, S.Lebegue, R.Ahuja, Physical Review B 2009, 79, 132102.

[222] T.B.Lee, M.L.McKee, Inorganic Chemistry 2009, 48, 7564.

[223] P.Loubeyre, R.Le Toullec, M.Hanfland, L.Ulivi, F.Datchi, D.Hausermann, Physical Review B 1998, 57, 10403.

[224] T.Ogitsu, E.Schwegler, F.Gygi, G.Galli, Physical Review Letters 2003, 91, 175502 .

[225] W.T.Klooster, T.F.Koetzle, P.E.M.Siegbahn, T.B.Richardson, R.H.Crabtree, Journal of the American Chemical Society 1999, 121, 6337.

[226] S.Trudel, D.F.R.Gilson, Inorganic Chemistry 2003, 42, 2814.

[227] Y.Lin, W.L.Mao, V.Drozd, J.Chen, L.L.Daemen, The Journal of Chemical Physics 2008, 129, 234509.

[228] C.Murli, Y.Song, The Journal of Physical Chemistry B 2009, 113, 13509.

[229] S.M.Lee, X.D.Kang, P.Wang, H.M.Cheng, Y.H.Lee, Chemphyschem 2009, 10, 1825 .

[230] N.J.Hess, M.E.Bowden, V.M.Parvanov, C.Mundy, S.M.Kathmann, G.K.Schenter, T.Autrey, The Journal of Chemical Physics 2008, 128, 034508. 
[231] M.Palumbo, F.J.Torres, J.R.Ares, C.Pisani, J.F.Fernandez, M.Baricco, Calphad 2007, 31, 457.

[232] V.Iosub, T.Matsunaga, K.Tange, M.Ishikiriyama, International Journal of Hydrogen Energy 2009, 34, 906.

[233] G.Sandrock, J.Reilly, J.Graetz, W.M.Zhou, J.Johnson, J.Wegrzyn, Applied Physics A: Materials Science and Processing 2005, 80, 687.

[234] J.Graetz, S.Chaudhuri, Y.Lee, T.Vogt, J.T.Muckerman, J.J.Reilly, Physical Review B 2006, 74, 214114.

[235] M.Tkacz, T.Palasyuk, J.Graetz, S.Saxena, Journal of Raman Spectroscopy 2008, 39, 922.

[236] M.Tkacz, S.M.Filipek, B.Baranowski, Polish Journal of Chemistry 1984, 57, 651.

[237] T.Kelkar, S.Pal, D.G.Kanhere, Chemphyschem 2008, 9, 928.

[238] T.Kelkar, D.G.Kanhere, S.Pal, Computational Materials Science 2008, 42, 510.

[239] P.Vajeeston, P.Ravindran, A.Kjekshus, H.Fjellvag, Physical Review Letters 2002, $89,175506$.

[240] J.F.Stampfer, C.E.Holley, J.F.Suttle, Journal of the American Chemical Society 2002, $82,3504$.

[241] M.Bortz, B.Bertheville, G.Bottger, K.Yvon, Journal of Alloys and Compounds 1999, 287, L4-L6.

[242] B.Baranowski, H.D.Hochheimer, K.Strossner, W.Honle, Journal of the LessCommon Metals 1985, 113, 341.

[243] A.Klaveness, P.Vajeeston, P.Ravindran, H.Fjellvag, A.Kjekshus, Physical Review $B$ 2006, 73, 094122 .

[244] E.Fuglein, U.Schubert, Chemistry of Materials 1999, 11, 865.

[245] A.Belsky, M.Hellenbrandt, V.L.Karen, P.Luksch, Acta Crystallographica Section B 2002, 58, 364.

[246] J.van Straaten, I.F.Silvera, Physical Review B 1987, 36, 9301.

[247] A.Zuttel, P.Wenger, S.Rentsch, P.Sudan, P.Mauron, C.Emmenegger, Journal of Power Sources 2003, 118, 1.

[248] Y.Fukai, "The metal-hydrogen system $\square$ basic bulk properties", 1993, Ch. 21. 
[249] A.El Gridani, R.Drissi El Bouzaidi, M.El Mouhtadi, Journal of Molecular Structure: THEOCHEM 2002, 577, 161.

[250] B.Huang, F.Gingl, K.Yvon, J.Rodriguez-Carvajal, Journal of Alloys and Compounds 1995, 227, 131.

[251] M.T.Hagstrom, J.P.Vanhanen, P.D.Lund, Journal of Alloys and Compounds 1998, $269,288$.

[252] R.K.Kremer, J.K.Cockcroft, H.Mattausch, A.Simon, G.J.Kearley, Journal of Physics: Condensed Matter 1994, 6, 4053.

[253] D.Kyoi, T.Sato, E.Ronnebro, N.Kitamura, A.Ueda, M.Ito, S.Katsuyama, S.Hara, D.Noreus, T.Sakai, Journal of Alloys and Compounds 2004, 372, 213.

[254] H.Hagemann, S.Gomes, G.Renaudin, K.Yvon, Journal of Alloys and Compounds 2004, 363, 129. 


\title{
APPENDIX
}

Review

\section{Structural stability of metal hydrides, alanates and borohydrides of alkali and alkali- earth elements: A review}

\author{
Lyci George*, Surendra K. Saxena
}

Department of Mechanical and Materials Engineering, Center for the Study of Matter at Extreme Conditions, Florida international University, Miami, FL 33199, United States

\section{A RTICLE IN FO}

Article history:

Received 25 February 2010

Received in revised form

16 March 2010

Accepted 17 March 2010

Available online 18 April 2010

Keywords:

Hydrogen storage materials

Hydrides

$\mathrm{X}$-ray diffraction

High- pressure

\begin{abstract}
A B S T R A C T
Alkali and alkali- earth metal hydrides have high hydrogen storage capacity, but high operation temperature hinders their use. The alanates and borohydrides of alkali and alkali- earth metals are widely studied because of their light weight and high hydrogen content. Borohydrides are highly stable and decompose only at elevated temperatures while alanates decompose in two steps. A detailed study of the properties of these hydrides is required for further understanding of their stability. A lot of thermodynamic information can be derived from investigation of materials under pressure or temperature. Structural measurements on the hydride compounds at ambient and high $\mathrm{P}-\mathrm{T}$ conditions result in a better understanding of the stability of the hydride structures and will assist us in the design of suitable storage materials with desired thermodynamic properties. The structural data are potential source of information regarding inter-atomic forces which determine pressure/temperature induced changes. During the past few years, structural stability of hydrides is widely investigated under pressure and temperature, both experimentally and theoretically. In this review we discuss structural phase transition and decomposition behavior of light metal hydrides, borohydrides and alanates of the elements that belong to first and second group in the periodic table.

Q 2010 Professor T. Nejat Veziroglu. Published by Elsevier Ltt. All rights reserved.
\end{abstract}

\section{Introduction}

The depletion of fossil fuel, increase in pollution and related environmental hazards require us to discover new energy sources. $\mathrm{H}_{2}$ gas is an efficient fuel with very high energy density. Storage is a challenging issue that cuts across production, delivery and commercial applications of hydrogen as energy carrier. Hydrogen stored in solid materials is a safe alternative to liquid or compressed form and convenient fuel with zero emission. Metals (eg. $\mathrm{LiH}, \mathrm{MgH}_{2}, \mathrm{NaBH}_{4}$, $\mathrm{Mg}\left(\mathrm{AlH}_{4}\right)_{2}, \mathrm{Li}_{2} \mathrm{NH}$ etc.) and high surface area sorbents (eg. clatherates, conducting polymers, nanotubes/fibers, aero gels etc.) are effective for solid state hydrogen storage.

Hydrides have high hydrogen density and light metal hydrides are good candidates for transportation. The transport sector requires not only cheap, safe and reliable way of storing hydrogen but also high storage capacity, fast kinetics and favorable themodynamics. A usable hydrogen storage candidate, which meets all the mentioned criteria, has yet to be discovered. A hydrogen storage material should release $\mathrm{H}_{2}$ at low temperature; it should have high volumetric density, rapid hydrogenation and dehydrogenation rates, high

\footnotetext{
- Corresponding author. Tel.: +1 305348 0540; fax: +1 3033483070 .

E-mail address: lgeoro01efiu.edu (L. George).

0360-3199/\$ - see front matter @ 2010 Professor T. Nejat Veziroglu. Published by Elsevier Ltd. All rights reserved. doi:10.1016/j.ijhydene.2010.03.078
} 


\title{
An extended high pressure-temperature phase diagram of $\mathrm{NaBH}_{4}$
}

Lyci George, ${ }^{\text {a) }}$ Vadym Drozd, Helene Couvy, Jiuhua Chen, and Surendra K. Saxena

Department of Mechanical and Materials Engineering, Center for the Study of Matter at Extreme Conditions, Florida International University, Miami, Florida 33199, USA

(Received 20 May 2009; accepted 2 August 2009; published online 20 August 2009)

\begin{abstract}
We have studied the structural stability of $\mathrm{NaBH}_{4}$ under pressures up to $17 \mathrm{GPa}$ and temperatures up to $673 \mathrm{~K}$ in a diamond anvil cell and formed an extended high $P-T$ phase diagram using combined synchrotron x-ray diffraction and Raman spectroscopy. Even though few reports on phase diagram of $\mathrm{NaBH}_{4}$ are found in current literature, up to our knowledge this is the first experimental work using diamond anvil cell in a wide pressure/temperature range. Bulk modulus, its temperature dependence, and thermal expansion coefficient for the ambient cubic phase of $\mathrm{NaBH}_{4}$ are found to be $18.76(1) \mathrm{GPa},-0.0131 \mathrm{GPa} \mathrm{K}^{-1}$, and $12.5 \times 10^{-5}+23.2 \times 10^{-8} \mathrm{~T} / \mathrm{K}$, respectively. We have also carried out Raman spectroscopic studies at room temperature up to $30 \mathrm{GPa}$ to reinvestigate the phase transitions observed for $\mathrm{NaBH}_{4}$. A comparative symmetry analysis also has been carried out for different phases of $\mathrm{NaBH}_{4} \cdot(-2009$ American Institute of Physics. [DOI: 10.1063/1.3211081]
\end{abstract}

\section{INTRODUCTION}

The structural investigation on metal borohydrides or alanates is interesting because of their high gravimetric hydrogen content. Sodium borohydride, $\mathrm{NaBH}_{4}$, is a potential hydrogen storage material and has a theoretical hydrogen storage capacity of $10.6 \mathrm{wt} \%$. Li et al., ${ }^{1}$ demonstrated $\mathrm{NaBH}_{4}$ slurry as an efficient way of application and generated hydrogen by the simple reaction $\mathrm{NaBH}_{4}+2 \mathrm{H}_{2} \mathrm{O}$ $\rightarrow \mathrm{NaBO}_{2}+4 \mathrm{H}_{2}$. Due to strong covalent and ionic bonding nature, dissociation temperatures of borohydrides are very high. The improvement of the hydride properties by catalytic addition requires better understanding of the phases and its phase stability. It is found that hydrogen desorption in $\mathrm{NaBH}_{4}$ can be enhanced by addition of $\mathrm{Pt}$ or $\mathrm{Ru}^{2}$ Under ambient conditions the $\mathrm{NaBH}_{4}$ has a cubic structure with space group $\mathrm{Fm}-3 \mathrm{~m}^{3.4}$ At low temperatures, below $190 \mathrm{~K}$, $\mathrm{NaBH}_{4}$ exists in a tetragonal structure. ${ }^{5,6}$ The well investigated high-pressure phases of $\mathrm{NaBH}_{4}$ at room temperature are of tetragonal- $P-42{ }_{1} c$ and orthorhombic-pnma structure which appears above 6.3 and $8.9 \mathrm{GPa}$, respectively. ${ }^{4}$ The previous reports suggest that the orthorhombic phase is stable in the pressure range of 8.9-30 GPa. Lee et al. ${ }^{7}$ studied the $a b$ initio structural stability of cubic and tetragonal phases of $\mathrm{NaBH}_{4}$ up to $30 \mathrm{GPa}$ and $4000 \mathrm{~K}$ but an experimental phase diagram is lacking in the current literature in these ranges. A low temperature phase diagram of $\mathrm{NaBH}_{4}$ was reported by Sundqvist and Andersson ${ }^{6}$ in the $P-T$ plane of $0-2 \mathrm{GPa}$ and $100-300 \mathrm{~K}$. In the phase diagram reported by Sundqvist et al., ${ }^{8}$ tetragonal to orthorhombic phase boundary of $\mathrm{NaBH}_{4}$ is marked in the range of 9-11 GPa.

Even though there are many reports on high pressure phase transitions some of them failed to observe a phase transition above $10.8 \mathrm{GPa}^{8}$ The $a b$ initio calculations by

a) Author to whom correspondence should be addressod. Electronic mail lgeor001@fiu.edu. Tel: +1305348 0540. FAX: +1 3033483070.
Araujo et al. ${ }^{9}$ showed a cubic to monoclinic transition at 19 $\mathrm{GPa}$ and to orthorhombic at $33 \mathrm{GPa}$. Through Raman spectroscopic studies, the same group observed a phase transition in the range of $\sim 10.8-14.8 \mathrm{GPa}$ and a completely new phase was formed above $15 \mathrm{GPa}$. However the $\mathrm{x}$-ray diffraction (XRD) experiments reported cubic to tetragonal transition at $6.3 \mathrm{GPa}$ and to orthorhombic at $8.9 \mathrm{GPa}^{4.10,11} \mathrm{Be}$ cause of these inconsistencies in reported transition pressures and phases, we have carried out in situ high $P-T$ structural measurements on $\mathrm{NaBH}_{4}$ both by XRD and Raman spectroscopy using diamond anvil cell (DAC) to obtain further understanding of its stability. Investigation of structural stability under elevated pressure and temperature can assist in the design of suitable storage materials with desired thermodynamic properties.

The high pressure structural behavior of alkali and alkaline earth metal borohydrides is widely investigated. The ambient phase of $\mathrm{LiBH}_{4}$ which has an orthorhombic (Pnma) structure transforms initially into a tetragonal (Ama2) at 1.2 $\mathrm{GPa}$ and then to a cubic $(F m-3 m)$ phase at $10 \mathrm{GPa}^{12,13}$ $\mathrm{KBH}_{4}$ exhibits structural phase transitions from a cubic $(\mathrm{Fm}-3 \mathrm{~m})$ to a tetragonal $(\mathrm{P}-421 \mathrm{C})$ phase at $3.8 \mathrm{GPa}$ and to an orthorhombic (Pnma) phase at $6.8 \mathrm{GPa}^{14} \mathrm{High}$ pressure phase transitions of alkali-metal borohydrides are found to be first order and fully reversible. In alkaline earth metal borohydrides, $\mathrm{Ca}\left(\mathrm{BH}_{4}\right)_{2}$ has an orthorhombic structure $(F 2 d d)$ and $\mathrm{Mg}\left(\mathrm{BH}_{4}\right)_{2}$ has a complex hexagonal unit cell with space group $P 6_{1}$ and both of them exhibit polymorphism. ${ }^{15,16}$ Therefore it is interesting to study the structural behavior of metal borohydrides under various conditions of pressures and temperatures.

Filinchuk et al. ${ }^{11}$ found that texture has much influence in the structure refinement of $\mathrm{NaBH}_{4}$. Theoretically predicted structures of borohydrides (Refs. 17 and 18) are found to be different from that of the experimentally observed ones. ${ }^{4,9,15,19}$ More theoretical and experimental investigations 


\title{
High-Pressure Investigation on Calcium Borohydride
}

\author{
Lyci George,* Vadym Drozd, and Surendra K. Saxena \\ Center for the Study of Matter at Extreme Conditions, Department of Mechanical and Materials Engineering, \\ Florida International University, Miami, Florida 33199
}

\author{
Elisa Gil Bardaji and Maximilian Fichtner \\ Forschungszentrum Karlsruhe GmbH, Institute of Nanotechnology, Karlsruhe-D 76021, Germany
}

Received: May 14, 2009; Revised Manuscript Received: July 9, 2009

\begin{abstract}
Structural properties of calcium borohydride, $\mathrm{Ca}\left(\mathrm{BH}_{4}\right)_{2}$, containing $\alpha$ and $\beta$ polymorphs are investigated under pressure by in situ synchrotron X-ray diffraction up to $13 \mathrm{GPa}$ and by Raman spectroscopy up to 25 $\mathrm{GPa}$. Results show that the $\beta$ phase transforms to a highly disordered structure above $10.2 \mathrm{GPa}$, whereas the $\alpha$ phase is stable in the pressure range studied. Rietveld analysis of the X-ray diffraction pattern collected at ambient conditions shows that the $\alpha$ phase is an orthorhombic $F 2 d d$ structure with lattice parameters $a=$ $8.82(7) \AA, b=13.05$ (10) $\AA, c=7.404(5) \AA$, and $V=851.8(10) \AA^{3}\left(64.13 \mathrm{~cm}^{3} / \mathrm{mol}\right)$. The $\beta$ phase was checked for all the suggested tetragonal structures, and the best match is obtained with space group $P 4_{2} / \mathrm{m}$; the lattice parameters are $a=6.88(4) \hat{A}, c=4.32(5) \AA$, and $V=204.82(18) \AA^{3}\left(61.68 \mathrm{~cm}^{3} / \mathrm{mol}\right)$. Pressure volume data obtained for $\alpha-\mathrm{Ca}\left(\mathrm{BH}_{4}\right)_{2}$ are fitted with the third-order Birch-Murnaghan equation of state that yields a bulk modulus value of $K_{0}=22.95$ (4) GPa with $K_{0}^{\prime}=2.63(0.5)$.
\end{abstract}

\section{Introduction}

Fuel cell technology requires hydrides of high hydrogen storage densities with moderate hydrogenation kinetics. Complex hydrides have high gravimetric storage capacities, and among them, $\mathrm{Ca}\left(\mathrm{BH}_{4}\right)_{2}$ and $\mathrm{Mg}\left(\mathrm{BH}_{4}\right)_{2}$ are promising materials because of the possibility of reversible hydrogen storage. ${ }^{1} \mathrm{Ca}\left(\mathrm{BH}_{4}\right)_{2}$ has a theoretical hydrogen storage capacity of $11.48 \mathrm{wt} \%$. In a proposed dehydriding reaction, a hydrogen release of $9.6 \mathrm{wt} \%$ is possible from $\mathrm{Ca}\left(\mathrm{BH}_{4}\right)_{2}$ with $\mathrm{CaH}_{2}$ and $\mathrm{CaB}_{6}$ as decomposition products. ${ }^{2}$ The thermal decomposition behavior of $\mathrm{Ca}\left(\mathrm{BH}_{4}\right)_{2}$ was investigated under hydrogen pressure by Kim et $\mathrm{al}^{3}$ and under vacuum or inert gas flow by Aoki et al. ${ }^{4}$ The results show that $\mathrm{Ca}\left(\mathrm{BH}_{4}\right)_{2}$ undergoes transformation from an orthorhombic to a tetragonal structure below $250{ }^{\circ} \mathrm{C}$ with a $5.9 \%$ hydrogen release. The structural investigations at different temperatures and pressures help to identify various polymorphs of the material that may have better thermodynamic stability. $\mathrm{Ca}\left(\mathrm{BH}_{4}\right)_{2}$ exhibits polymorphism depending on the synthesis and processing conditions and also transforms to different phases at various temperatures. ${ }^{1.5-9}$ Filinchuk et al. ${ }^{6}$ observed three polymorphs of $\mathrm{Ca}\left(\mathrm{BH}_{4}\right)_{2}$ in the temperature range of $30-600^{\circ} \mathrm{C}$, which are orthorhombic $\alpha(F 2 d d)$, tetragonal $\alpha^{\prime}(\bar{I} 2 d)$, and tetragonal $\beta$ $(P \overline{4})$ structures. Below $300^{\circ} \mathrm{C}, \alpha$ and $\alpha^{\prime}$ polymorphs completely transform to the $\beta$ phase. The second-order transition from $\alpha$ to $\alpha^{\prime}$ is found to be dependent on heating rate/sample synthesis and processing conditions. Two more possible structures of the $\alpha$ phase are $C 2 / c$ and $F d d d$ suggested by Majzoub et al. ${ }^{8}$ and Miwa et al., ${ }^{2}$ respectively. Buchter et al. ${ }^{9}$ and Lee et al. ${ }^{7}$ independently indexed a tetragonal phase in $P 4_{2} / m$ space group that seems to be more reliable than $P \overline{4}$, according to our results. An orthorhombic $\gamma$ phase of space group pbca was observed by Riktor et al., 5,8 which transforms to the $\beta$ phase on heating to $290{ }^{\circ} \mathrm{C}$ and again to an unknown phase $\delta$ around $330{ }^{\circ} \mathrm{C}$.

* Corresponding author. Phone: +1 305348 0540. Fax: +1 303348 3070. E-mail: 1geor001@ fiu.edu
Catalyst addition is also found to influence the formation of different phases in $\mathrm{Ca}\left(\mathrm{BH}_{4}\right)_{2}$ by Ronnebro et al. ${ }^{1}$ Although there are many studies about the structural stability of $\mathrm{Ca}\left(\mathrm{BH}_{4}\right)_{2}$ at high temperatures, investigation under pressure is lacking in the current literature.

Structural phase transitions under high pressure or temperature are very likely in alkali and alkaline-earth metal borohydrides. The ambient-pressure orthorhombic (Pnma) polymorph of $\mathrm{LiBH}_{4}$ transforms initially at $1.2 \mathrm{GPa}$ into a tetragonal phase of Ama 2 space group and then to a cubic $(F m \overline{3} \mathrm{~m})$ phase at 10 $\mathrm{GPa}^{10,11} \mathrm{KBH}_{4}$ exhibits structural phase transitions from a cubic $(F m \overline{3} m)$ to a tetragonal $\left(P \overline{4} 2_{1} c\right)$ phase at $3.8 \mathrm{GPa}$ and to an orthorhombic (Pnma) phase at $6.8 \mathrm{GPa}^{12} \mathrm{High}$-pressure phase transitions of alkali metal borohydrides are found to be firstorder and fully reversible. $\mathrm{Mg}\left(\mathrm{BH}_{4}\right)_{2}$ has a complex hexagonal unit cell with space group $P 6_{1}$ that undergoes transition to a simple $P_{6}$ structure around $3.3 \mathrm{GPa}^{13}$ Similar to $\mathrm{Ca}\left(\mathrm{BH}_{4}\right)_{2}$, magnesium borohydride also exhibits polymorphism. Therefore, it is interesting to study the high-pressure behavior of $\mathrm{Ca}\left(\mathrm{BH}_{4}\right)_{2}$.

Majzoub et al. ${ }^{8}$ observed the formation of an $\sim 50 \% \beta$ phase after mechanical milling of $\alpha-\mathrm{Ca}\left(\mathrm{BH}_{4}\right)_{2}$, which was believed to be because of the combined effects of pressure and temperature. They calculated the bulk modulus of various polymorphs of $\mathrm{Ca}\left(\mathrm{BH}_{4}\right)_{2}$ using the first-principles method, and it was $\sim 15$ $\mathrm{GPa}$. The same group also predicted the transition of $\alpha$ to $\beta$ phase at $5.3 \mathrm{GPa}$. Even though there is much theoretical and high-temperature work, a high-pressure experimental study on $\mathrm{Ca}\left(\mathrm{BH}_{4}\right)_{2}$ is not found in the current literature. Factors affecting the polymorphism of $\mathrm{Ca}\left(\mathrm{BH}_{4}\right)_{2}$ are not yet known completely. Structural measurements at high pressure can assist in designing a suitable storage material with desired thermodynamic properties. In this report, we present results from in situ X-ray diffraction and Raman spectroscopic studies on $\mathrm{Ca}\left(\mathrm{BH}_{4}\right)_{2}$ at high pressures to understand the structural stability of its polymorphs and to verify the results of theoretical calculations.

10.1021/jp904513j CCC: $\$ 40.75$ @ 2009 American Chemical Society Published on Web 07/24/2009 


\title{
Bulk modulus and thermal expansion coefficient of mechano- chemically synthesized $\mathrm{Mg}_{2} \mathrm{FeH}_{6}$ from high temperature and high pressure studies
}

\author{
Lyci George*, Vadym Drozd, Andriy Durygin, Jiuhua Chen, Surendra K. Saxena \\ Center for the Study of Matter at Extreme Conditions, Department of Mechanical and Materials Engineering, Florida International University,
} Miami, FL 33199, USA

A R T I C L E I N F O

Article history:

Received 31 December 2008

Received in revised form

14 February 2009

Accepted 16 February 2009

Available online 12 March 2009

\section{Keywords:}

Hydride

Hydrogen storage material

High pressure and temperature

Bulk modulus

Thermal expansion

\begin{abstract}
A B S T R A C T
$\mathrm{Mg}_{2} \mathrm{FeH}_{6}$ was synthesized by ball milling $\mathrm{MgH}_{2}$ and Fe (2:1 molar ratio) mixture for $72 \mathrm{~h}$ followed by heating at $400^{\circ} \mathrm{C}$ under $\mathrm{H}_{2}$ pressure. The hydride formation, its structure and homogeneity were investigated by scanning electron microscopy, X-ray diffraction, transmission electron microscopy and Raman spectroscopy. High pressure in situ synchrotron X-ray diffraction and Vienna ab initio simulation were used to determine bulk modulus of the sample. The bulk modulus of $\mathrm{Mg}_{2} \mathrm{FeH}_{6}$ was found to be 75.4(4) GPa by optimized experiment and $76.3 \mathrm{GPa}$ by theoretical simulation. From high temperature in situ X-ray diffraction study the volumetric thermal expansion coefficient of $\mathrm{Mg}_{2} \mathrm{FeH}_{6}$ was found to be $\alpha_{v}=5.85(3) \times 10^{-5}+7.47(7) \times 10^{-8}\left(\mathrm{~T}-\mathrm{T}_{0}\right) /{ }^{\circ} \mathrm{C}$. Decomposition of $\mathrm{Mg}_{2} \mathrm{FeH}_{6}$ was observed at $425^{\circ} \mathrm{C}$ and the decomposition products were $\mathrm{Mg}$, Fe and $\mathrm{H}_{2}$.

(c) 2009 International Association for Hydrogen Energy. Published by Elsevier Ltd. All rights
\end{abstract} reserved.

\section{Introduction}

Magnesium iron hydride $\left(\mathrm{Mg}_{2} \mathrm{FeH}_{6}\right)$ is widely studied because of its possible application for hydrogen and thermo-chemical heat storage [1,2]. $\mathrm{Mg}_{2} \mathrm{FeH}_{6}$ has the highest volumetric density of hydrogen $\left(150 \mathrm{kgH}_{2} \mathrm{~m}^{-3}\right)$ among all the hydrides investigated so far and has a gravimetric hydrogen density of $5.4 \mathrm{wt} \%$ $\mathrm{H}_{2}$. Moreover, $\mathrm{Mg}_{2} \mathrm{FeH}_{6}$ is more economical for many applications compared to other hydrides because of the availability and the low cost of component metals $\mathrm{Mg}$ and $\mathrm{Fe}$. The hydride has a $\mathrm{K}_{2} \mathrm{PtCl}_{6}$-type cubic structure with a space group of Fm$3 \mathrm{~m}$, composed of tetrahedral sites of $\mathrm{Mg}^{2+}$ ions and octahedral $\left(\mathrm{FeH}_{6}\right)^{4-}$ complexes. Magnetic and Mossbauer spectroscopy measurements revealed a diamagnetic nature for the $\mathrm{Mg}_{2} \mathrm{FeH}_{6}$ suggesting a low-spin iron(II) configuration [3].
Various research groups have carried out investigations on the synthesis of $\mathrm{Mg}_{2} \mathrm{FeH}_{6}$ [4-12]. Herrich et al., [5] studied the influence of milling conditions and temperature on the hydride formation and obtained a $90 \%$ yield of $\mathrm{Mg}_{2} \mathrm{FeH}_{6}$. Among the compositions investigated a ratio 2:0.57 of $\mathrm{MgH}_{2}$ to Fe exhibited good results. Desorption behavior of $\mathrm{Mg}_{2} \mathrm{FeH}_{6}$ was investigated based on synthesis conditions and unreacted $\mathrm{Fe}$ content. The observed desorption temperatures were $355^{\circ} \mathrm{C}[5]$ and $428^{\circ} \mathrm{C}[6]$. The desorption mixture of $\mathrm{Mg}_{2} \mathrm{FeH}_{6}$ reversibly absorbs hydrogen to form the hydride at $550^{\circ} \mathrm{C}$ and 60 bar $\mathrm{H}_{2}$ pressure. The presence of $\mathrm{Fe} / \mathrm{MgH}_{2}$ enhances the $\mathrm{H}_{2}$ desorption, however the temperature does not enhance the synthesis rate. Hout et al., [6] reported better reversibility in $\mathrm{Mg}_{2} \mathrm{FeH}_{6}$ synthesized by ball milling of $2 \mathrm{MgH}_{2}+\mathrm{Fe}$ mixture than sintering of $2 \mathrm{Mg}+\mathrm{Fe}$ under hydrogen.

\footnotetext{
* Corresponding author. Tel.: +1 305348 0540; fax: +1 3033483070

E-mail address: lgeor001@fiu.edu (L. George).
}

0360-3199/\$ - see front matter ๑ 2009 International Association for Hydrogen Energy. Published by Elsevier Ltd. All rights reserved. doi:10.1016/j.ijhydene.2009.02.043 


\title{
Structural Phase Transitions of $\mathrm{Mg}\left(\mathrm{BH}_{4}\right)_{2}$ under Pressure
}

\author{
Lyci George, * Vadym Drozd, and Surendra K. Saxena \\ Center for the Study of Matter at Extreme Conditions, Department of Mechanical and Materials Engineering, \\ Florida International University, Miami, Florida 33199
}

Elisa Gil Bardaji and Maximilian Fichtner

Institute of Nanotechnology, Forschungszentrum Karlsruhe GmbH, Karlsruhe-D 76021, Germany

Received: September 3, 2008; Revised Manuscript Received: November 4, 2008

\begin{abstract}
The structural stability of $\mathrm{Mg}\left(\mathrm{BH}_{4}\right)_{2}$, a promising hydrogen storage material, under pressure has been investigated in a diamond anvil cell up to $22 \mathrm{GPa}$ with combined synchrotron X-ray diffraction and Raman spectroscopy. The analyses show a structural phase transition around $2.5 \mathrm{GPa}$ and again around $14.4 \mathrm{GPa}$. An ambient-pressure phase of $\mathrm{Mg}\left(\mathrm{BH}_{4}\right)_{2}$ has a hexagonal structure (space group $P 6_{1}, a=10.047(3) \AA, c=$ $36.34(1) \AA$, and $V=3176(1) \AA^{3}$ at $0.2 \mathrm{GPa}$ ), which agrees well with early reports. The structure of highpressure phase is found to be different from reported theoretical predictions; it also does not match the hightemperature phase. The high-pressure polymorph of $\mathrm{Mg}\left(\mathrm{BH}_{4}\right)_{2}$ is found to be stable on decompression, similar to the case of the high-temperature phase. Raman spectroscopic study shows a similarity in high-pressure behavior of as-prepared $\mathrm{Mg}\left(\mathrm{BH}_{4}\right)_{2}$ and its high-temperature phase.
\end{abstract}

\section{Introduction}

Metal hydrides provide a safe way to store hydrogen with high density. The transport sector requires not only a light, cheap, safe, and reliable hydrogen storage method but also high hydrogen storage capacity, fast kinetics, and favorable thermodynamics. A usable hydrogen storage candidate, which meets all the mentioned criteria, has yet to be discovered. Light metal borohydrides are attractive due to their high gravimetric and volumetric hydrogen densities compared to other complex hydrides. Among them, magnesium borohydride, $\mathrm{Mg}\left(\mathrm{BH}_{4}\right)_{2}$, is a promising lightweight solid-state hydrogen storage material with a theoretical hydrogen capacity of $14.8 \mathrm{wt} \%$.

The thermodynamical properties of $\mathrm{Mg}\left(\mathrm{BH}_{4}\right)_{2}$ were examined by several groups, and it was found that the reaction enthalpy and entropy should give 1 bar of equilibrium pressure for hydrogen at room temperature. ${ }^{1-4}$ The low enthalpy of decomposition suggests a possibility of reversible hydrogen storage in this compound. ${ }^{3,4.12}$ However, it seems as if there were high kinetic barriers involved in the hydrogen desorption, leading to a high operation temperature $\left(>270^{\circ} \mathrm{C}\right)$, poor kinetics, and difficulty in rehydrogenating the desorption product. The possible decomposition steps for $\mathrm{Mg}\left(\mathrm{BH}_{4}\right)_{2}$ may be $\mathrm{Mg}\left(\mathrm{BH}_{4}\right)_{2}$ $\rightarrow \mathrm{MgH}_{2}+2 \mathrm{~B}+3 \mathrm{H}_{2} \rightarrow \mathrm{Mg}+2 \mathrm{~B}+4 \mathrm{H}_{2} \rightarrow \mathrm{MgB}_{2}+4 \mathrm{H}_{2}{ }^{2}$ The investigation of Ozolins et al. ${ }^{18}$ led to the conclusion that thermodynamically reversible hydrogen storage is possible in $\mathrm{Mg}\left(\mathrm{BH}_{4}\right)_{2}$ but it is kinetically hindered. Moreover, a calcium analogue of $\mathrm{Mg}\left(\mathrm{BH}_{4}\right)_{2}$ has been demonstrated recently to show reversible hydrogen storage properties. ${ }^{5-7}$ To improve the hydrogen absorption/desorption properties of $\mathrm{Mg}\left(\mathrm{BH}_{4}\right)_{2}$, additives such as Ti are explored. ${ }^{2}$ To verify such a phenomenon in $\mathrm{Mg}\left(\mathrm{BH}_{4}\right)_{2}$, further research is inevitable by theoretical calculations of thermodynamic properties, which in turn requires proper structural data in a wide range of temperatures and pressures which should be obtained from X-ray diffraction. Moreover,

* To whom correspondence should be addressed. Phone: (305) 348-0540. Fax: (303) 348-3070. E-mail: lgeor001@ fiu.adu. destabilization of metal borohydrides was suggested ${ }^{3.9}$ to be achieved by application of high pressure via formation of short $\mathrm{H} \cdot \cdot \mathrm{H}$ distances and deformation of tetrahedral $\mathrm{BH}_{4}$ anions.

There are many reports on the synthesis and crystal structure of $\mathrm{Mg}\left(\mathrm{BH}_{4}\right)_{2}{ }^{1-3,10-17} \mathrm{Jae}-\mathrm{Hyuk}$ Her et al. ${ }^{11}$ reported that the crystal structure of $\mathrm{Mg}\left(\mathrm{BH}_{4}\right)_{2}$ is hexagonal $\left(P 6_{1}\right)$ below $180^{\circ} \mathrm{C}$ and above this temperature it transforms to an orthorhombic phase (Fddd) which is stable up to the compound decomposition temperature of $340^{\circ} \mathrm{C}$. The $P 6_{1}$ structure of $\mathrm{Mg}\left(\mathrm{BH}_{4}\right)_{2}$ with 30 formula units ( 330 atoms) in a unit cell contains 5 symmetryindependent $\mathrm{Mg}^{2+}$ cations and $10 \mathrm{BH}_{4}^{-}$anions. Hydrogen coordination for $\mathrm{Mg}^{2+}$ is 8 , and locally the $\mathrm{Mg}^{2+}$ network resembles an amorphous state. ${ }^{13}$ The density of the lowtemperature phase of $\mathrm{Mg}\left(\mathrm{BH}_{4}\right)_{2}$ is found to be $0.78 \mathrm{~g} / \mathrm{cm}^{3}$, whereas that of the high-temperature phase is $0.76 \mathrm{~g} / \mathrm{cm}^{3}$. The structure of the high-temperature phase was found to be coherent in the $b c$ plane but not in the $a$ direction. The bonding in $\mathrm{Mg}\left(\mathrm{BH}_{4}\right)_{2}$ is partially covalent, similar to that of $\mathrm{MgH}_{2}$, but it has a higher bond strength than $\mathrm{MgH}_{2} \cdot{ }^{17}$ The $\mathrm{B}-\mathrm{H}$ interaction in $\mathrm{BH}_{4}^{-}$tetrahedra is ionocovalent, and that between $\mathrm{Mg}$ and $\mathrm{H}$ is ionic. ${ }^{12}$ The hydrogen and $\mathrm{BH}_{4}{ }^{-}$coordination of the $\mathrm{Mg}^{2+}$ ion is strongly related to its ionic radius/ionic character, which may be a cause of the complex bonding nature of $\mathrm{Mg}^{2+}$ ions. First-principal density functional theory (DFT) calculations failed to predict the ambient-pressure structure of $\mathrm{Mg}\left(\mathrm{BH}_{4}\right)_{2}$ due to its complexity. ${ }^{3,12,18}$

High-pressure structural studies of a number of metal borohydrides have been reported in the past few years. Calcium borohydride, $\mathrm{Ca}\left(\mathrm{BH}_{4}\right)_{2}$, has an orthorhombic structure similar to that of the high-temperature phase of $\mathrm{Mg}\left(\mathrm{BH}_{4}\right)_{2}$ but a comparatively simple unit cell. ${ }^{19}$ The ambient-pressure orthorhombic (Pnma) polymorph of $\mathrm{LiBH}_{4}$ transforms at $1.2 \mathrm{GPa}$ into a tetragonal phase (Ama2), and at about $10 \mathrm{GPa}$ a second transformation into a cubic $(F m \overline{3} m)$ phase takes place. ${ }^{89}$ The ambient-pressure fcc phase of $\mathrm{NaBH}_{4}$ at room temperature transforms into a tetragonal $P \overline{4} 2_{1} c$ phase near $6 \mathrm{GPa}$ and then into an orthorhombic Pnma phase above $8 \mathrm{GPa}^{20}$ Similarly to

\section{$10.1021 / j p 807842 \mathrm{t}$ CCC: $\$ 40.75$ C 2009 American Chemical Society
Published on Web $12 / 09 / 2008$}




\title{
Principal component analysis on properties of binary and ternary hydrides and a comparison of metal versus metal hydride properties
}

\author{
Lyci George $^{\mathrm{a}, *}$, Ross Hrubiak ${ }^{\mathrm{a}}$, Krishna Rajan ${ }^{\mathrm{b}}$, Surendra K. Saxena ${ }^{\mathrm{a}}$ \\ a Center for the Study of Matter at Extreme Conditions, Department of Mechanical and Materials Engineering, Florida International University, Miami, FL 33199, USA \\ ${ }^{b}$ Department of Materials Science and Engineering, Iowa State University, Ames, IA 50011, USA
}

\section{A R T I C L E I N F O}

\section{Article history:}

Received 17 September 2008

Received in revised form

22 November 2008

Accepted 27 November 2008

Available online 6 December 2008

\section{Keywords:}

Metal hydrides

Energy storage materials

Thermodynamic properties

Metals and alloys

\section{A B S T R A C T}

Principal component analysis (PCA) is used to investigate interrelationship among material properties of hydrides. Property data which consist of $\sim 200$ compounds (binary and ternary metal hydrides) were analyzed. A comparative study was carried out among the metal properties with that of their hydrides. The observed decrease or increase of entropy, molar volume and specific heat of hydrides from that of the metals can be attributed to hydrogen bond formation, charge transfer and corresponding change in crystal structure.

(c) 2008 Elsevier B.V. All rights reserved.

\section{Introduction}

Storage is a challenging issue that cuts across production delivery and end-use applications of hydrogen as energy carrier. Hydrogen stored in hydrides is safe and has a high hydrogen density. Hydrides are promising materials both as fuel in automobiles and many others such as in rechargeable batteries, fuel cells, refrigerator, heat storage, nuclear industry, sensors, optical switch, and hydrogen purification. The light element hydrides are good candidates for transportation [1]. The transport sector requires not only cheap, safe and reliable way of storing hydrogen but also high storage capacity, fast kinetics and favorable thermodynamics. A practical hydrogen storage candidate, which meets all the mentioned criteria, has yet to be discovered. Main problems of hydrides are weight, volume, cost, safety, efficiency, refueling time, durability, etc. [2]. Therefore, it is important to study their properties to design suitable hydrides for specific applications.

Material informatics is a developing area in material science in which a new knowledge system is built by collecting and classifying information with the help of calculations and databases. If experience and knowledge, captured and preserved in a database format, it helps to investigate new materials efficiently. The abundance of parameters causes difficulties in the interpretation of their relation. Many datum sets can be correlated to define a single materia

* Corresponding author. Tel.: +1 305348 0540; fax: +1 3033483070 . E-mail addresses: Igeor001@fiu.edu, lycigv@gmail.com (L. George).

0925-8388/\$ - see front matter @ 2008 Elsevier B.V. All rights reserved. doi: $10.1016 /$ j.jallcom.2008.11.137 property with the help of a mathematical technique. The main challenge in this field is the difficulty in correlating many properties of different set of materials. Most of the classical analytical methods for finding characteristics of data set are very laborious and time consuming.

Lot of experimental and theoretical work has been done on hydrides in search of a suitable material. A detailed hydride research database is required for proper retrieval of information already available. We collected data from available journal publications and constructed property database (which has to be published soon somewhere else). Data sets in the hydride database can be correlated to define a single material property using available mathematical technique such as principal component analysis (PCA) which is a widely used tool for dimensionality reduction and for visualization of variable patterns. In this work we have considered only binary (of the form $\mathrm{MH}_{x}$, where $x=1,2$ or 3 and $\mathrm{M}$ is a metal) and ternary (of the form $\mathrm{A}_{a} \mathrm{~B}_{b} \mathrm{H}_{x}$, where $a, b$ and $x$ are integers and $\mathrm{A}$, $B$ are metals) metal hydrides. We have excluded other hydrides due to the presence of large missing data and associated error on analysis. This work investigates correlation among material properties and compares various metal hydride properties with corresponding metallic properties.

\section{Data}

We have collected properties which include but not limited to specific heat $(C p)$, entropy $(S)$, molar volume $(V)$ and hydrogen weight percentage (HWt\%) of metal hydrides as well as the sum of 
VITA

\section{LYCI GEORGE}

\section{EDUCATION AND PROFESSIONAL EXPERIENCE}

$1997-2000$

Bachelor of Science (B.Sc.) in Physics, Mahatma Gandhi University, Kottayam, India

2000-2002

Master of Science (M.Sc.) in Physics, Mahatma Gandhi University, Kottayam, India

2003-2005

Master of Technology (M.Tech.) in Materials Science, Indian Institute of Technology, Kanpur, India

Junior Research Fellow, University Grant Commission (UGC), India

2005-2006

Senior Research Fellow, UGC and Indian Institute of Technology, Kanpur, India

2006-2010

Research Assistant (2006-2007),

CDAC (Carnegie/ DOE alliance center) student (2007-2008),

Dissertation Evidence Acquisition Fellow (2008-2009) and

Dissertation Year Fellow (2009-2010),

Florida International University, Miami, FL

\section{PUBLICATIONS AND PRESENTATIONS}

1. Gajbhiye, N.S., Pandey, P. K., George, L. and Kumar, A. "Characterization of nano structured PZT prepared by chemical routes", Journal of Nanoscience and Nanotechnology 7 (2007) 1975-1979.

2. Gajbhiye, N.S., Pandey, P. K., George, L. and Kumar, A. "Dielectric properties of nanostructured PZT prepared by chemical routes", Defense Science Journal, 57(2007) 61-68.

3. Gajbhiye, N.S., Pandey, P. K., George, L. and Kumar, A. "Thermal, structural and electrical studies of nanostructured PZT synthesized by low temperature technique", Thermans (2006) 284-286.

4. Gajbhiye, N.S., Pandey, P. K., George, L. and Kumar, A. "Studies on homogeneous lead zirconate titanate powder synthesized by hydroxide co-precipitation", Proceedings of the DAE Solid State Physics Symposium (2005) 267-268. 
5. George, L., Drozd, V., Chen, J, Couvy, H. and Saxena, S. K. “An extended high pressure- temperature phase diagram of $\mathrm{NaBH}_{4}$ ", Journal of Chemical Physics 131 (2009) 074505.

6. George, L., Drozd, V., Bardaji, E. G., Fichtner, M. and Saxena, S. K. "High pressure investigation on calcium borohydride", Journal of Physical Chemistry: C 113 (2009) 15087-15090.

7. George, L., Drozd, V., Durygin, A., Chen, J. and Saxena, S. K. "Bulk modulus and thermal expansion of mechano-chemically synthesized $\mathrm{M}_{\mathrm{g} 2} \mathrm{FeH}_{6}$ from high temperature and high pressure studies" International Journal of Hydrogen Energy 34 (2009) 3410-3416.

8. George, L., Drozd, V., Bardaji, E. G., Fichtner, M. and Saxena, S. K. "Structural phase transitions of $\mathrm{Mg}\left(\mathrm{BH}_{4}\right)_{2}$ under pressure", Journal of Physical Chemistry: C 113 (2009) 486-492.

9. George, L., Hrubiak, R., Rajan, K. and Saxena, S. K. "Principal component analysis on properties of binary and ternary hydrides and a comparison of metal versus metal hydride properties", Journal of Alloys and Compounds 478 (2009) 731-735.

10. George, L. and Saxena, S. K. "Structural stability of metal hydrides, alanates and borohydrides of alkali and alkali- earth elements: A review", International Journal of Hydrogen Energy 35 (2010) 5454-5470.

11. Hrubiak, R., George, L., Saxena S. K. and Rajan, K. “A materials database for exploring material properties", Journal of Metals 61(2009) 59-62.

12. Vennila, R. S., Drozd, V., George, L., Saxena, S.K., Liermann, P., Liu, H., Stowe, C. A., Berseth, P., Anton D. and Zidan, R. "Structural study of ball-milled sodium alanate under high pressure", Journal of Alloys and Compounds 473 (2009) 576-578.

Also attended and presented the work at 10 national and international conferences during the dissertation period. 